\title{
Advances in Understanding the Sea Ice Floe Size Distribution
}

by

Laetitia Roach

A thesis submitted to Victoria University of Wellington

in fulfilment of the requirements for the degree of Doctor of Philosophy

Victoria University of Wellington

2019 



\begin{abstract}
Sea ice is a critical component of the polar climate system that is tightly coupled to the ocean and atmosphere. It is highly heterogeneous, composed of discrete floes which range in size across space and time. In this thesis, I use a combination of modelling and observational approaches to investigate how different physical processes determine the distribution of sea ice floe sizes. I construct the first global model that simulates floe sizes arising from the interaction of different physical processes. Floe sizes are modified by lateral melt, lateral growth, freezing together of floes and wave-ice interactions. By grounding process descriptions in underlying physics, observations of individual processes can be used to constrain model parameters. In light of the sparseness of floe size observations, I developed a novel methodology to constrain previously-unobserved floe freezing processes from in-situ observations. Results from global coupled sea ice-ocean model simulations are used to quantify the relative impacts of different processes on spatial and seasonal variability in the floe size distribution, providing hypotheses that could be tested by observational campaigns in the future. Under transient historical forcing, the model suggests that the fragmentation of Arctic sea ice has significantly increased over the satellite era.
\end{abstract}

I also seek to improve understanding of feedbacks between sea ice floe size and the polar climate system. A fragmented ice cover exposes more ice area on the sides of floes to the ocean than sheet ice, promoting lateral melt, which reduces surface albedo. Conducting a statistical analysis of current climate models shows that inclusion of a lateral melt parametrization improves simulation of sea ice concentration relative to observations. However, calculation of lateral melt using the model for prognostic simulation of the sub-grid-scale floe size distribution results in little or no enhancement of lateral melt at a hemispheric scale compared to a simple parametrization, although it is likely to be important at smaller spatial and shorter temporal scales. The new model opens up the possibility of coupling sea ice and ocean surface wave models and of including floe size dependence in other processes, such as form drag, sea ice dynamics, ocean eddies and ocean-atmosphere heat transfer, which may result in significant impacts for polar climate. 


\section{ACKNOWLEDGEMENTS}

First and foremost, many thanks to Sam Dean for his advice, enthusiasm and scientific guidance throughout this thesis. Sam, you've been a truly excellent supervisor. Thanks also to my second supervisor James Renwick for his mentorship and for convincing me to make the move to New Zealand, a decision that I am very happy to have made. My co-authors on various parts of this thesis have been a real pleasure to work with, as well as providing valuable scientific stimulus. Cecilia Bitz really helped the model development work get off the ground when she hosted me in Seattle and has shared her wealth of expertise throughout the project. Chris Horvat has been a great collaborator; together we've bounced ideas around and shared some of the ups and downs of the $\mathrm{PhD}$ journey. Maddie Smith, my room-mate on the PIPERS cruise to the Ross Sea, provided great observational know-how and infectious enthusiasm during work on our paper. I look forward to future projects together with you all.

I am grateful to colleagues at NIWA who have generously given their time to help me, particularly Erik Behrens, Jonny Williams, Richard Gorman and Alena Malyarenko. Big thanks to Alison Kohout for getting me to Antarctica, a fantastic experience. My Masters supervisor, Simon Tett, sparked my interest in and taught me a great deal about climate modelling. I have benefited from mentorship and encouragement from others throughout my education at Rogart Primary, Golspie High School and the University of Edinburgh. I have also benefited from the work done by senior women in science to break down barriers and make the scientific community a more inclusive place. The visibility of women in sea ice research inspired me to think that this was a field where I could contribute.

I could not have completed this thesis without encouragement and moral support from friends and family. Thanks to all of you-old friends, new friends, Mum, Dad, and especially Patrik.

This research was funded by the New Zealand Royal Society Marsden Fund (contract number VUW1408) and supported by the National Institute of Water and Atmospheric Research (NIWA). 


\section{Contents}

Abstract

Acknowledgements

Table of Contents $\quad$ iv

1 Introduction 1

2 Background 5

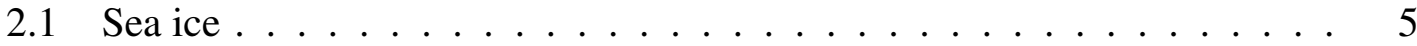

2.2 Sea ice observations . . . . . . . . . . . . . . 8

2.3 Sea ice and the climate system . . . . . . . . . . . . . . 11

2.4 Climate models . . . . . . . . . . . . . . . . . . 14

2.5 Development of sea ice models . . . . . . . . . . . . . . 15

2.5.1 Ice thickness distribution . . . . . . . . . . . . . 16

2.5.2 Thermodynamics ................... 19

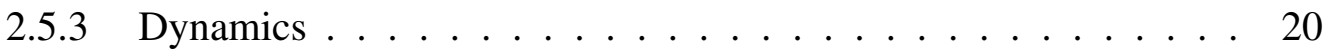

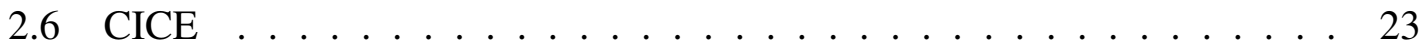

2.7 Sea ice floe size distribution . . . . . . . . . . . . . 26

2.7.1 Definition of the floe size distribution . . . . . . . . . . 26

2.7.2 Observations of floe sizes . . . . . . . . . . . 27

2.7.3 Processes driving floe sizes . . . . . . . . . . . . . 32

2.7 .4 Importance of floe sizes $\ldots \ldots . \ldots 32$

2.7.5 Modelling floe size . . . . . . . . . . . . . . 33

3 Consistent biases in Antarctic sea ice concentration simulated by climate models

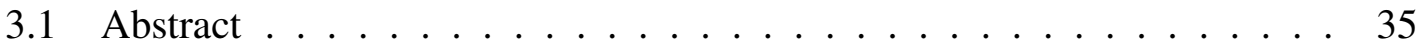




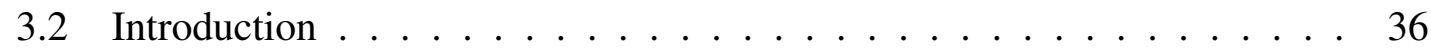

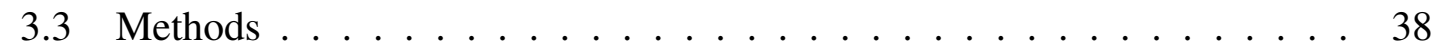

3.3.1 CMIP5 Models . . . . . . . . . . . . . . . . . . . . . 38

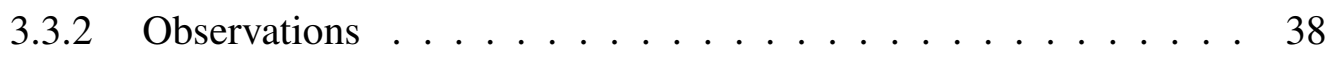

3.3 .3 Metrics . . . . . . . . . . . . . . . 42

3.3.4 Coupled ocean-sea ice model . . . . . . . . . . . . . . . 44

3.4 Results . . . . . . . . . . . . . . . . . . . . 46

3.5 Impact of floe size $\ldots \ldots \ldots \ldots \ldots \ldots \ldots \ldots$

3.6 Discussion . . . . . . . . . . . . . . . . . 62

4 An emergent sea ice floe size distribution in a global coupled ocean-sea ice $\begin{array}{ll}\text { model } & 67\end{array}$

4.1 Abstract . . . . . . . . . . . . . . . . . . . 67

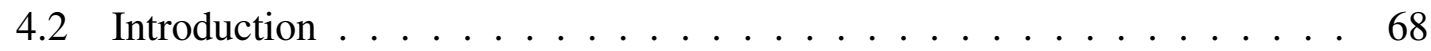

4.3 Model . . . . . . . . . . . . . . . . . . . . . . . . . . 69

4.3.1 Standard model . . . . . . . . . . . . . . . . . . . . . . . . . 69

4.3.2 The joint floe size and ice thickness distribution . . . . . . . . 71

4.3.3 Thermodynamics . . . . . . . . . . . . . . . . 73

4.3 .4 Wave fracture . . . . . . . . . . . . . . 76

4.4 Results . . . . . . . . . . . . . . . . . . . . 78

4.5 Discussion . . . . . . . . . . . . . . . . . 88

4.6 Conclusions $\ldots \ldots \ldots \ldots \ldots \ldots \ldots \ldots \ldots \ldots \ldots \ldots \ldots \ldots \ldots$

5 Quantifying growth of pancake sea ice floes using images from drifting buoys 95

5.1 Abstract . . . . . . . . . . . . . . . . . 95

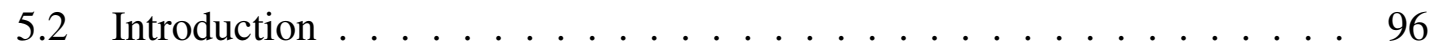

5.3 Data . . . . . . . . . . . . . . . . . . . 99

5.4 Methods . . . . . . . . . . . . . . . . . . 102

5.5 Floe size evolution . . . . . . . . . . . . . . . . . 105

5.6 Wave constraints on floe growth $\ldots \ldots \ldots \ldots \ldots \ldots$

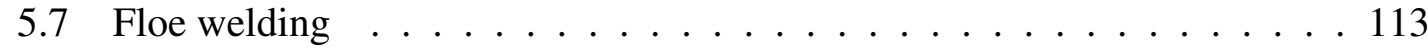

5.8 Lateral growth of floes . . . . . . . . . . . . . . . . 115

5.9 Discussion . . . . . . . . . . . . . . . . . . . . 117

5.10 Conclusions $\ldots \ldots \ldots \ldots \ldots \ldots$ 
6 Variability in floe size distribution simulated by a model with improved representation of wave-limited floe sizes

6.1 Introduction . . . . . . . . . . . . . . . . . . . . . . . 123

6.2 Methods . . . . . . . . . . . . . . . . . . . . . . . . . 124

6.2 .1 Metrics . . . . . . . . . . . . . . . . . . . . . . 124

6.2.2 Model configuration . . . . . . . . . . . . . . . . . 125

6.2 .3 Wave-limited floe sizes . . . . . . . . . . . . . . . . . 126

6.3 Results \& Discussion . . . . . . . . . . . . . . . . . . . . . . 128

6.3.1 Impact of wave-limited ice floe sizes . . . . . . . . . . . . . 128

6.3.2 Seasonal evolution of Arctic floe perimeter . . . . . . . . . 132

6.3.3 Impacts on sea ice concentration over the satellite era . . . . . . 133

6.3.4 Impacts of Arctic climate change on the FSD . . . . . . . . . 137

6.4 Conclusions . . . . . . . . . . . . . . . . . . . . . . . . . . . . . 139

7 Discussion 141

7.1 What are the processes that control the sea ice floe size distribution? . . . 141

7.2 What is the relationship between sea ice floe size and the polar climate system? . . . . . . . . . . . . . . . . . . . . . . . . . 144

7.3 How do we integrate observations and models to better understand sea ice? 146

8 Conclusions

A Sensitivity to floe welding parameter 155

B Model adjustment for transient simulations 157

C Sensitivity to floe size categories 159

$\begin{array}{ll}\text { Bibliography } & 162\end{array}$ 


\section{Chapter 1}

\section{Introduction}

The rapid decline of Arctic sea ice in recent decades is a particularly visible and striking indicator of climate change. Although a large portion of sea ice melts and re-grows every year, the area of perennial sea ice cover in the Northern Hemisphere has more than halved since the beginning of the observational record in 1979 (Vaughan et al., 2013). The Arctic may become seasonally ice-free by the middle of this century (Collins et al., 2013). Meanwhile, the areal coverage of Antarctic sea ice has varied substantially but with a slightly positive trend, although it is expected to decrease by the end of this century (Ferreira et al., 2015).

Reductions in sea ice have direct impacts on society, such as loss of habitat for wildlife and increased human access to the polar regions. Perhaps more importantly, changes in sea ice have wide-ranging implications for Earth's climate. The thin layer of bright sea ice on the ocean surface acts to reflect incoming solar radiation and to insulate the relative warmth of polar oceans from the cold atmosphere above, while brine rejected during freezing of sea ice drives movement of water masses in the ocean. Sea ice therefore plays a role in local and remote weather systems (Vihma, 2014), ocean circulation (Pellichero et al., 2018), and the sensitivity of the climate system to increased warming (Caldeira and Cvijanovic, 2014).

Up close, sea ice is a fascinating and complex medium which can vary dramatically in its appearance over mere metres. It is composed of floes, discrete masses of frozen seawater, which range in both horizontal size and thickness. Lateral sea ice floe sizes span orders of magnitude - from small floes on the order of centimetres to welded floes 
kilometres in diameter in the interior of the ice cover-and evolve in space and time. Sea ice cover is affected by a multitude of physical processes on different timescales, including melt on the top, sides and bottom of floes; movement and collisions driven by the dynamical forces exerted by currents, winds and waves; and new ice formation dependent on ocean conditions.

Mathematical models are essential tools for understanding the physics of this highlycomplex system, as well as providing projections and analysis of past and future change. Sea ice models describe the key dynamical and thermodynamical processes that determine sea ice evolution. Naturally, the full complexity of the Earth system cannot be represented in a computer model, so not all processes can be included, and those that are must be parametrized in some way. Within a model grid cell, which can be $50-100 \mathrm{~km}$ across for climate-scale studies, pieces of sea ice are typically assumed to have the same size horizontally, with only the ice thickness varying.

The simplification of sea ice floe size to a single constant value may have adverse consequences for the representation of physical processes in sea ice models. The sizes of floes determine the total perimeter of ice floes exposed to the ocean, and therefore the amount of melt that occurs around floe edges, or 'lateral melt' (Steele, 1992). Lateral melt directly reduces sea ice concentration, in contrast to basal and surface melt which only reduce sea ice concentration once sea ice thickness has been reduced to zero. Reductions in concentration allow greater solar input in to the upper ocean, facilitating further melt, a positive feedback loop that may be important for sea ice decay (Asplin et al., 2014).

Besides their effect on lateral melt, the different sizes of sea ice floes contribute to the overall roughness of the sea ice surface, causing friction for atmospheric and oceanic flow and affecting air-sea energy exchanges (Steele et al., 1989; Lüpkes et al., 2012). Mechanically, smaller floes are likely to have more rapid, collisional interactions, while larger floes deform slowly with frictional interactions at floe edges (Shen et al., 1987; Feltham, 2005; De Silva et al., 2015). The spatial distribution of floes determines the distribution of open water areas within the ice pack, through which large transfers of heat can occur (Marcq and Weiss, 2012). Sea ice floes modulate ocean surface waves as they travel through ice, viscously dissipating wave energy at small floe sizes and scattering wave energy at larger floe sizes (Montiel et al., 2016). More directly, predictions of floe sizes are 
relevant for polar operations, such as design of icebreakers, engineering calculations of ice impact forces on coastal structures, and forecasts for shipping.

As well as enabling predictions of floe size and more realistic representation of climaterelevant physical processes, models describing the sea ice floe size distribution could be used to test our understanding of the underlying physics determining floe sizes. While some studies have measured the sea ice floe size distribution from aerial photos or satellite imagery (e.g. Wang et al. 2016), observations are limited in spatial and temporal coverage. Although fracture of sea ice by ocean surface waves is thought to be important in determining floe sizes (e.g. Toyota et al. 2006), its impact has not been quantified, and the processes driving floe sizes are poorly understood.

Accordingly, this thesis presents work that makes advances in answering the following research questions:

1. What are the processes that control the sea ice floe size distribution?

2. What is the relationship between sea ice floe size and the polar climate system?

3. How do we integrate observations and models to better understand sea ice?

In Chapter 2, I provide background reading on sea ice, its role in the climate system, sea ice models and a review of studies on floe size. Chapter 3 analyses a suite of global climate model simulations relative to satellite sea ice observations, examining whether models exhibit consistent biases and discussing potential causes. Only the Southern Hemisphere is considered in this chapter, as model agreement with observations is generally poorer in the Antarctic than the Arctic, and few Antarctic-focused assessments exist in the literature. This has been published as

- Roach, L. A., S. M. Dean, and J. A. Renwick (2018a), Consistent biases in Antarctic sea ice concentration simulated by climate models, The Cryosphere, 12(1), 365383, doi:10.5194/tc-12-365-2018.

Motivated by this, Chapter 4 describes implementation of a sub-grid-scale sea ice floe size distribution in a global sea ice-ocean model. We present results from climatological simulations demonstrating the impact of an evolving floe size distribution on sea ice concentration and thickness. This has been published as 
- Roach, L. A., C. Horvat, S. M. Dean, and C. M. Bitz (2018b), An emergent sea ice floe size distribution in a global coupled ocean-sea ice model, Journal of Geophysical Research: Oceans, 123(6), 4322-4337, doi:10.1029/2017JC013692.

Chapter 4 highlighted the lack of studies on floe freezing processes, motivating an observational study in Chapter 5. This tests our physical description of floe freezing processes against observations, and has been published as

- Roach, L. A., M. M. Smith, and S. M. Dean (2018c), Quantifying growth of pancake sea ice floes using images from drifting buoys, Journal of Geophysical Research: Oceans, 123(4), 2851-2866, doi:10.1002/2017JC013693.

Findings in Chapter 5 supported the introduction of a new model parametrization to describe floe sizes when ice is initially formed. In Chapter 6, I use a global sea ice-ocean model with this improved description of floe freezing to quantify the role of different processes in driving the floe size distribution, and to investigate change in the floe size distribution in a changing climate. In Chapter 7, I discuss how the different chapters address the three research questions, and conclude in Chapter 8. 


\section{Chapter 2}

\section{Background}

This chapter provides background reading on sea ice, its role in the climate system, climate models and development of sea ice models. I conclude with a review of studies on sea ice floe size, which sets the scene for the original pieces of research that follow.

\subsection{Sea ice}

A distinctive feature of the polar regions is sea ice, a thin solid layer of frozen sea water. Sea ice floats in the ocean due to the unique properties of water. Water is made up of two positively-charged hydrogen ions and a negatively-charged oxygen ion. As water is cooled, the motion of water molecules slows down and, approaching the freezing point, a crystalline structure maintained by hydrogen bonds begins to form. Below the 'maximum density temperature' $\left(4^{\circ} \mathrm{C}\right.$ for fresh water), the effect of negative oxygen ions repelling one another overcomes thermal contraction, and the molecules cannot contract further. The density of water therefore decreases as water is cooled below the maximum density temperature to the freezing point (Greenwood and Earnshaw, 2012). The crystalline structure of the solid phase (ice) is less dense than liquid water, so it can float. This is in contrast to most other substances, which only contract and become denser as they are cooled.

The structural properties of water determine circulation in bodies of fresh and salt water. Above $4{ }^{\circ} \mathrm{C}$, if a body of freshwater is cooled from above, convection will occur as the top layer becomes denser than the water below and sinks. If the top layer is cooled below $4{ }^{\circ} \mathrm{C}$, its density decreases, suppressing convection and stratifying the water col- 
umn. In the case of seawater, the presence of salt ions delays the formation of crystalline structures as temperature decreases. This lowers the freezing point of seawater to around -1.8 to $-2{ }^{\circ} \mathrm{C}$ and the maximum density temperature to below the freezing point (Weeks and Ackley, 1986) for typical polar ocean salinities (30 to $35 \mathrm{psu}$, Zweng et al. 2013). Therefore, seawater density always increases with decreasing temperature during the liquid phase, unlike freshwater. If seawater is cooled from above, the top layer becomes denser than the seawater below, and it is prone to convection throughout cooling. The presence of a vertical salinity gradient in the Arctic and Antarctic Oceans stabilizes the water column so that convection in this upper 'mixed' layer is generally limited to the upper 30-50 m (Talley et al., 2011). Exceptions occur in high-salinity regions where sea ice formation rejects dense brine and promotes deep convection, such as on the continental margins around Antarctica (Killworth, 1983).

Several metres of the upper layer must be lowered to or below the freezing point for ice formation to occur (Weeks and Ackley, 1986), which is achieved more quickly in stably-stratified freshwater than convecting seawater. Once the upper oceanic mixed layer is cold enough, small needle-like crystals called frazil (e.g. Fig. 2.1a) form, expelling salt. Salt ions cannot fit into the crystal structure of ice and are expelled during freezing. Although most is rejected into the ocean beneath, some liquid brine remains trapped in pockets within the ice. In very calm, cold conditions, frazil crystals will join together into a smooth unbroken surface of sheet ice called nilas (e.g. Fig. 2.1d). Nilas are initially almost transparent, with thicknesses of around $1-10 \mathrm{~cm}$. As heat is conducted away from the relatively warm ocean, the ice thickens by congelation growth, where ice crystals grow downwards from the established ice cover into the water. Congelation growth slows down as the ice thickens, since the heat flux is inversely proportional to ice thickness.

When turbulence is present, a common occurrence in the open ocean, frazil crystals are stirred through a thin portion of the upper ocean layer. When this 'soupy mess' (Weeks and Ackley, 1986) reaches a crystal volume fraction of around $40 \%$ it transitions to a more solid form (Martin and Kauffman, 1981), and can reach thicknesses of up to a metre (Weeks and Ackley, 1986). Winds and waves may herd the crystals into circular aggregations called pancakes, which are initially slushy but solidify into distinguishable shapes with upturned edges from repeated collisions with one another (e.g. Fig. 2.1b). Pancakes 


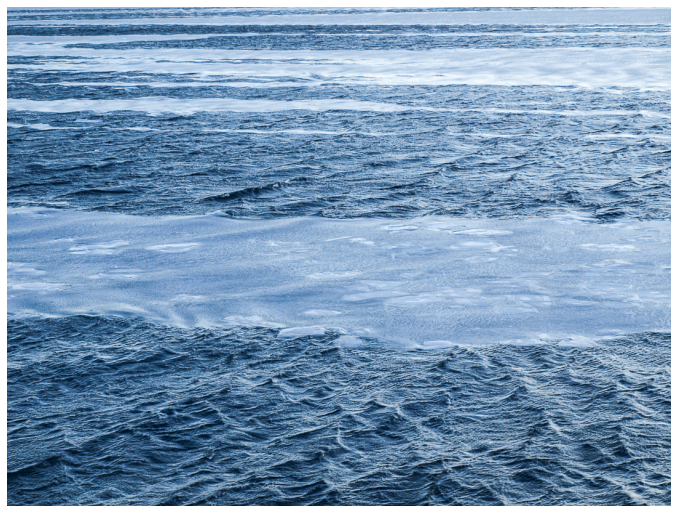

(a) Frazil crystals

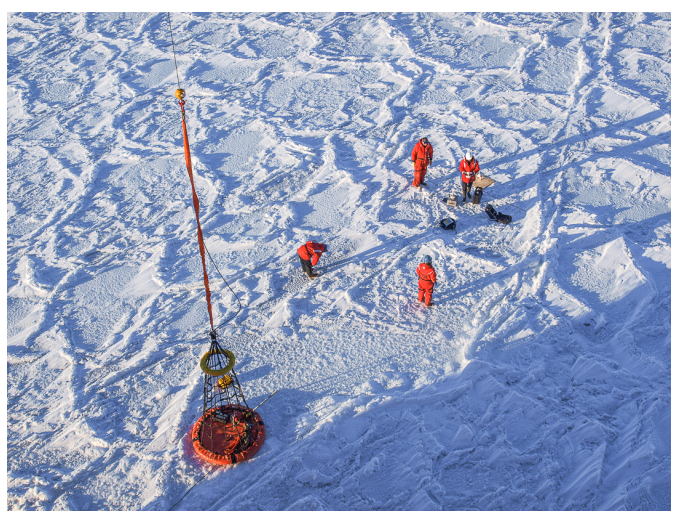

(c) Cemented pancakes

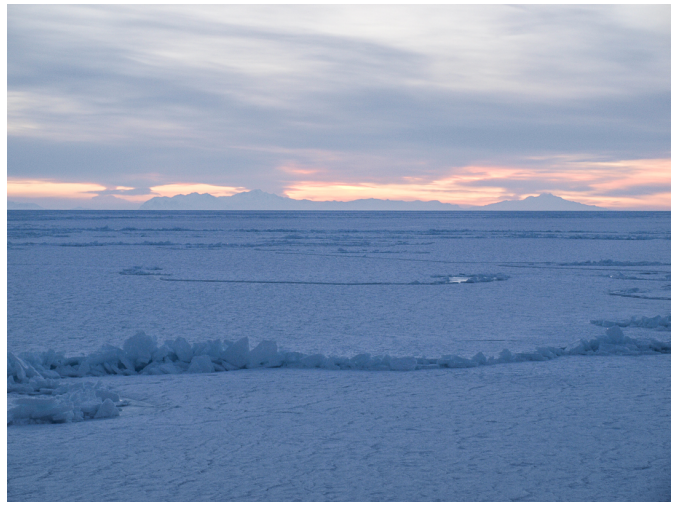

(e) Pack ice with a small ridge

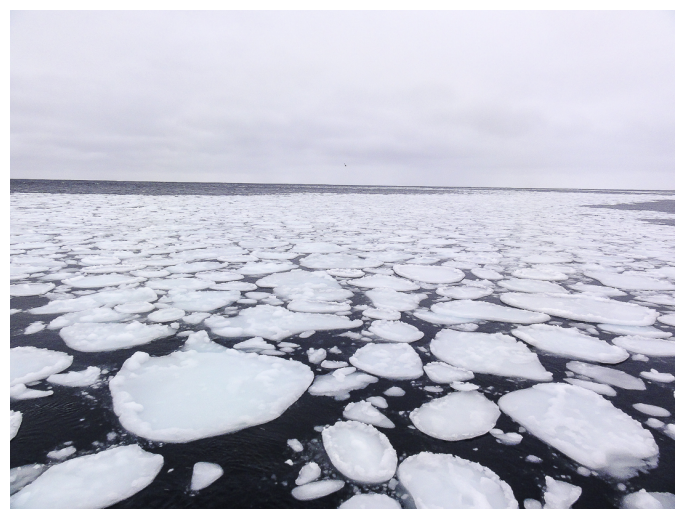

(b) Pancakes

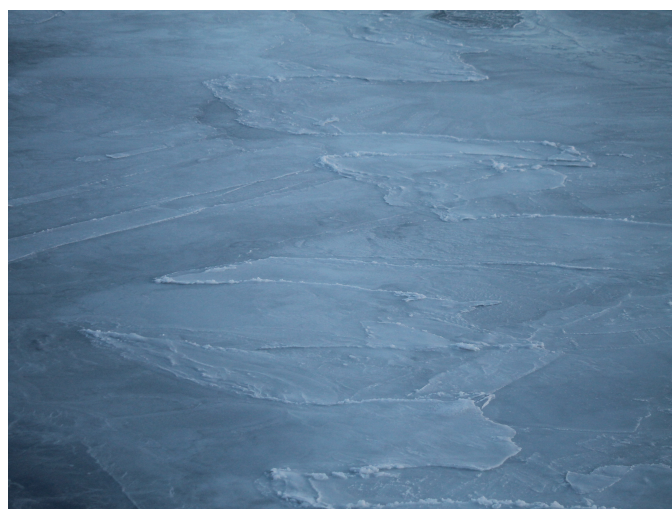

(d) Nilas

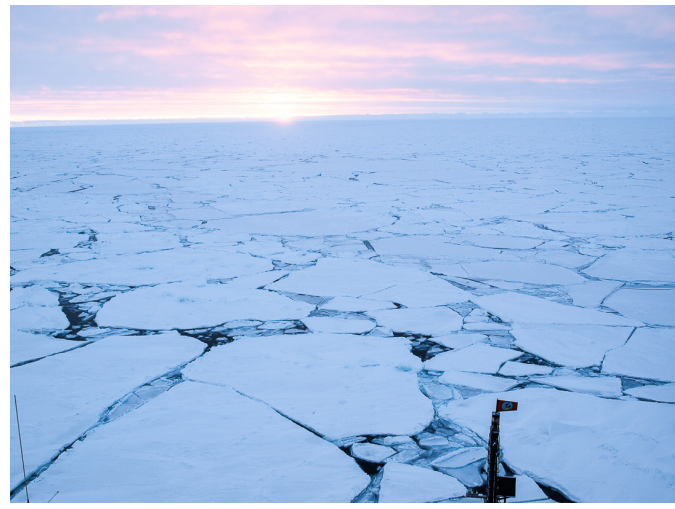

(f) Fractured ice

Figure 2.1: Photographs of sea ice types in the Ross Sea, Antarctica taken by the author during the PIPERS cruise (April - June 2017). 
can expand laterally and freeze together (see Chapter 5 for more details) and may also be joined together by nilas if conditions quiesce. Sheets of welded or 'cemented' pancakes (e.g. Fig. 2.1c) can then thicken by congelation growth. Often the presence of visible pancake edges gives a clue as to their initial formation. The different growth processes result in sea ice of quite distinct appearances.

Further heterogeneity in the appearance of ice is introduced by snow, melt processes and mechanical behaviour. Snow falls on sea ice, increasing the already high reflectivity of the surface and the insulation between the ocean and atmosphere. The weight of snow can push the ice surface below sea level, allowing snow-ice to form during freezing conditions. In the summer, sea ice ablation occurs at the bottom and around the sides of floes. The ocean may be warm enough to facilitate the phase change from solid to liquid. If the ocean temperature is below the melting point of the sea ice, the phase change requires transport of salt to the phase boundary, and the ice dissolves rather than melts (Woods, 1992). Melt occurs on the top surface of the ice when air temperatures exceed $0^{\circ} \mathrm{C}$, as is common in the Arctic but less so in the Antarctic, allowing heterogeneous melt ponds to accumulate in depressions on the ice surface. Sea ice melt is a source of freshwater which acts to stabilize the water column.

Winds and currents move sea ice around, sometimes pushing floes into one another causing ridging, where ice piles up as floes collide (e.g. Fig. 2.1e), or rafting, where floes slide over one another, during convergent motion. Areas of open water, called leads, are opened up in the ice pack as a result of divergent motion and shear, as well as melting. If high strain rates are exerted on ice floes by the passage of ocean surface waves, floes fracture into smaller pieces (e.g. Fig. 2.1f). The existence of these different processes and their interaction result in dramatic variation in the appearance of sea ice over small spatial scales.

\subsection{Sea ice observations}

Sea ice concentration, the fraction of a spatial area covered by sea ice, can be inferred from the brightness temperature of Earth's surface as measured by passive microwave radiometers. Observational uncertainty exists in sea ice concentration products due to differences between the algorithms used to convert passive-microwave signals into sea 
ice concentration (see Chapter 3 for more details). Differences between observational datasets are smaller when considering quantities integrated over large areas such as hemispheric sea ice extent, which is defined as the area of all grid cells in each hemisphere with a fractional coverage of sea ice greater than $15 \%$ (Notz, 2014).

These observations show that the sea ice surrounding Antarctica exhibits a stronger seasonal cycle than Arctic sea ice, with Southern Hemisphere sea ice extent varying from around 3 to 18 million $\mathrm{km}^{2}$ each year (Parkinson and Cavalieri, 2012), compared to 5 to 15 million $\mathrm{km}^{2}$ in the Northern Hemisphere on average (Cavalieri and Parkinson, 2012). The two poles have different geographies that influence their behaviours. The Arctic is largely surrounded by land, so ice can build up and form areas of thick ridging. The Antarctic is much more exposed to the open ocean; its sea ice is generally more mobile and thinner (Washington and Parkinson, 2005).

The 40-year record of satellite sea ice concentration observations reveals striking sea ice trends in the Arctic as the climate has warmed. Over this period, the largest reduction in sea ice extent has occurred in the month of September, with a trend of $-12.8 \pm$ $2.3 \%$ per decade over 1979-2018 relative to the 1981-2010 average (Fig. 2.2a, Fetterer et al. 2017). The largest trends in the Antarctic also occurred during its fall season, but show a small increase, of $2.8 \pm 3.7 \%$ per decade in March over 1979-2018 relative to the 1981-2010 average (2.2b, Fetterer et al. 2017). Southern Hemisphere sea ice extent has high variability, with a recent record high winter maximum in 2014 followed by a second lowest summer minimum in 2016 and record low winter maximums in 2016 and 2017 (Fig. 2.2b).

The thickness of sea ice is needed to obtain a picture of sea ice in three dimensions. Since 2010, satellite radar altimeter has provided Arctic-wide measurements (in autumn, winter and spring) of sea ice freeboard, which is the distance between the water level and the top surface of sea ice. This can be converted to sea ice thickness by assuming that floes are floating in hydrostatic equilibrium, such that the upwards pressure gradient is balanced by the downwards force of gravity (Tilling et al., 2018). The conversion requires estimates of snow depth, which are the greatest source of uncertainty in this data (Tilling et al., 2018) and remain a significant problem for Antarctic retrievals. Thickness measurements have also been made by submarine, upward looking sonar, airborne elec- 

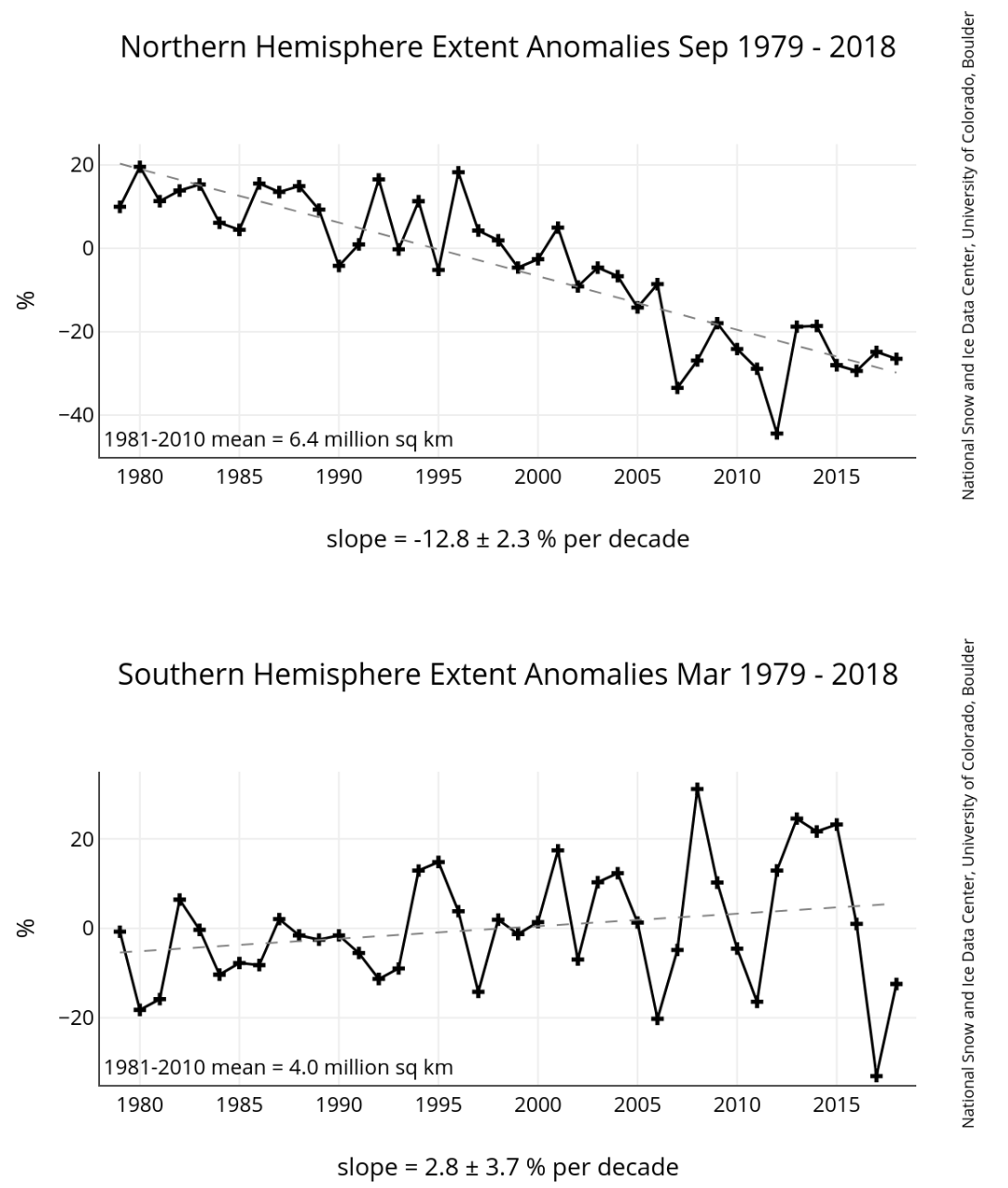

Figure 2.2: Monthly ice extent anomalies plotted as a time series of percent difference between the extent for the month in question and the mean for that month based on the January 1981 to December 2010 data. The anomaly data points are plotted as plus signs and the trend line is plotted with a dashed grey line. Caption and figures reproduced from Fetterer et al. (2017).

tromagnetic soundings and in-situ drill holes, which do not provide a continuous record and are limited in spatial coverage, particularly for the Antarctic. Reanalysis data, from models that assimilate some observations to produce a hindcast, are often used to augment the sea ice thickness record (e.g. Schweiger et al. 2011; Massonnet et al. 2013). Combining methods, it is estimated that Arctic summer sea ice volume has declined by three quarters since the 1980s (Vaughan et al., 2013). It is not known how Antarctic sea ice thickness has changed (Vaughan et al., 2013), although reanalyses suggest a slight increase in thickness where concentrations have increased (Massonnet et al., 2013; Holland et al., 2014). 
Other variables characterizing sea ice that can be observed by satellite include sea ice age (e.g. Maslanik et al. 2011), melt pond coverage (e.g. Rösel and Kaleschke 2012), and floe size (e.g. Hwang et al. 2017). Of these, records of floe size are the most sparse in time and space. See Subsec. 2.7 for a review of floe size observations.

\subsection{Sea ice and the climate system}

Changes in sea ice have global relevance, due to the role of sea ice in the climate system. The climate system consists of five main components: the atmosphere, hydrosphere, cryosphere, land surface and biosphere (Baede et al., 2001). There are many complex and non-linear interactions between the various components on different timescales. The atmosphere reacts to changes in boundary conditions on short timescales of hours to days, sea ice over days to months, the deep ocean over centuries and Antarctic ice sheets over millennia (Neelin, 2010). Non-linearity, inherent in the equations for fluid flow used to describe the atmosphere and ocean, results in chaotic behaviour in some elements of the climate system. The climate system generates a region's climate, often defined as the mean and variability of weather on decadal timescales for the region (Baede et al., 2001).

Statistically significant changes in the mean or variability of climate are referred to as 'climate change' (Baede et al., 2001). Such changes may result from forcing external to the system or variability internal to the system. Incoming radiation from the Sun is the main driver of our climate system and must be balanced by outgoing radiation. The average top-of-atmosphere net radiation is zero in an equilibrium climate state. Changes in solar radiation as well as changes in the properties of the atmosphere which determine the amount of infrared radiation emitted back to space (such as the composition of trace gases which absorb and emit infrared radiation) alter this balance (Baede et al., 2001). Changes in climate also arise from internal natural variability. One example is the North Atlantic Oscillation, a pattern of sea level pressure differences between the Icelandic Low and the Azores High which fluctuates without any particular periodicity. Besides external forcings and internal variability, the climate system is further complicated by feedbacks, whereby the result of a process may amplify or reduce its original effect.

A well-known and long-recognized positive feedback relates to sea ice albedo, the 
amount of radiation reflected from the surface of sea ice. When reflective sea ice melts, it exposes a darker ocean surface, permitting more absorption of solar radiation, which in turn enhances ice melt. Early studies hypothesized that this effect would cause global warming to first manifest in the polar regions (Manabe and Stouffer, 1980). As Arctic sea ice has declined, expanding areas of open ocean absorb more heat during the summer, which must be released to the atmosphere in fall before ice can refreeze. This plays a significant role in the 'Arctic amplification' of atmospheric warming, at a rate around double that of the mid-latitudes (Serreze et al., 2009; Screen and Simmonds, 2010).

Besides this reflective effect of sea ice, which acts to cool the ocean during the summer months by preventing it from absorbing solar radiation, during the winter months sea ice insulates the warm ocean and prevents it losing heat to the atmosphere. A thinner sea ice cover provides less insulation, cooling the ocean, and driving more ice production. This is a negative feedback known as the ice-thickness-ice-growth feedback, which can be more simply expressed as 'thin ice grows faster than thick ice' (Bitz and Roe, 2004). A related dynamical feedback also occurs as thin ice converges and deforms more easily than thicker ice, creating open water and allowing more ice production (Vavrus, 1999). These stabilizing processes act against the runaway loss of polar ice cover, known as 'small ice cap instability' (Brooks, 1925), that could be expected from the ice-albedo feedback.

Sea ice plays a major role in the transformation of ocean water masses, bodies of water with identifiable physical properties that distinguish them from the surrounding waters. The formation of sea ice, which rejects brine, creates a denser surface water layer and impacts ocean stratification. In the Southern Ocean sea ice region, freshwater fluxes associated with sea ice are the dominant component in surface buoyancy flux estimates (Pellichero et al., 2018). Formation, transport and melt of sea ice redistributes freshwater from high to low latitudes, driving meridional overturning ocean circulation (Pellichero et al., 2018). Sea ice formation is essential for production of Antarctic Bottom Water (Ohshima et al., 2016), the densest water layer in the deep ocean and a sink for heat and carbon dioxide. A recent modelling study suggested that projected reduced Antarctic sea ice formation and associated increased stratification would allow warm Circumpolar Deep Water to enhance basal melting of ice shelves, with consequences for global sea level rise (Naughten et al., 2018). 
In the Arctic, buoyancy fluxes associated with sea ice decline could explain the reduction in strength of the Atlantic Meridional Overturning Circulation (AMOC) (Sévellec et al., 2017), which is believed to have slowed down by about $15 \%$ since the mid twentieth century (Caesar et al., 2018). Increased precipitation, river run-off and melt from the Greenland Ice Sheet also contribute to surface freshening, and the exact contribution of sea ice is unclear. The AMOC transports warm waters polewards, and so reductions in the AMOC would lead to regional climate changes in the mid-latitudes. Sea ice decline has also been linked to weather changes in the mid-latitudes, for example colder winters over Northern Eurasia and eastern North America (Cohen et al., 2012), wetter European summers (Screen, 2013) and more persistent weather extremes (Francis and Vavrus, 2012). However, the large inter-annual variability, short observational record and limited mechanistic understanding of these potential remote responses currently preclude robust conclusions on the role of sea ice (Vihma, 2014). Locally, however, sea ice change has been to shown to cause changes in the near-surface atmosphere, particularly during winter (Alexander et al., 2004; Rinke et al., 2006) and with largest impacts in the marginal ice zone (Rinke et al., 2006).

The tight coupling of sea ice to the ocean and atmosphere complicates investigation of causal relationships. The vertical scale of sea ice $(\mathcal{O}(\mathrm{m}))$ is much smaller than the vertical scale of the ocean $\left(\mathcal{O}\left(10^{3} \mathrm{~m}\right)\right)$ and atmosphere $\left(\mathcal{O}\left(10^{4} \mathrm{~m}\right)\right)$, and it responds quickly to the other climate system components. In the Antarctic, atmospheric patterns and associated winds are thought to drive most inter-annual sea ice variability, through advection of warm and moist or cold and dry air masses, as well as impacts on sea ice motion (see review in Hobbs et al. 2016). The then-record low Antarctic sea ice extent in 2016 coincided with record atmospheric circulation anomalies (Turner et al., 2017). The Antarctic Circumpolar Current (ACC) constrains the location of the sea ice edge in the Southern Hemisphere (Nghiem et al., 2016). In the Arctic, the sea ice edge is constrained by the presence of continents (Eisenman, 2010), and the perennial sea ice cover is increasingly vulnerable to a warm reservoir of Atlantic water beneath it (Polyakov et al., 2017). These are just some examples of coupling between the sea ice, ocean and atmosphere. 


\subsection{Climate models}

These kind of climate system relationships can be investigated using global climate models (GCMs), numerical representations of the climate system based on the physical, chemical and biological properties of its components (Flato et al., 2013). Essentially based on conservation of mass, energy and momentum on a rotating sphere, they model interactions between the various land, sea, air and ice processes. Equations are converted to finite difference form for numerical solutions. Models are forced by energy from the sun and can incorporate other external forcings such as carbon dioxide emissions, volcanoes and aerosols.

Resulting variables are simulated on coordinate grids spanning the Earth and with a number of vertical levels. Grid resolution is a trade-off between accuracy and computational cost. Models exist at all scales of complexity, with the most advanced solving the full discretized equations of motion. These advanced models include coupling between the atmosphere and ocean and incorporate a sea ice component.

There are three fundamental sources of uncertainty in climate model projections:

- Scenario uncertainty: future external forcing is not known

- Internal variability: inherent noise within the climate system due to its chaotic nature

- Modelling uncertainties: missing physics and structural and parameter uncertainties

Clearly, future forcings on the climate system such as the quantity of emitted greenhouse gases, changes in solar radiation and volcanic eruptions are unknown. We do not consider scenario uncertainty in this thesis and discuss only historical simulations. Like the natural world, complex climate models exhibit considerable internal variability on a variety of timescales. Minute differences in atmospheric initial conditions can have a substantial impact on climate trajectories, yet still represent the same 'model climate' (Kay et al., 2015). Internal variability is a significant issue when comparing climate models to observations, as discussed in Chapter 3. The use of a coupled ocean-sea ice model with prescribed atmospheric forcing rather than coupled to a dynamical atmosphere model, as in Chapters 3 and 4, imposes one realization of atmospheric variability. This strongly 
constrains the system and limits variability in the ocean and sea ice response.

Modelling uncertainty is the error source most relevant to this thesis. Many different models exist: the Intergovernmental Panel on Climate Change (IPCC) compared over 50 models from 20 modelling groups in its latest model inter-comparison project (CMIP5, Taylor et al. 2012) over 2010 to 2014. Models may incorporate different processes and produce simulations in different ways. All models have to make some approximations to represent complex physical processes at discrete points and times. Most global climate models have horizontal grid scales of order $100 \mathrm{~km}$ or more. This means that, for example, small-scale turbulent motion in the ocean is poorly represented, although it transports a significant amount of ocean heat (e.g. Zhao et al. 2018). Models try to address issues like this by using parametrizations, whereby processes which occur on a sub-modelresolution spatial scale are simplified by a single number, a statistical representation, or an assumed functional dependence on large-scale resolved variables (Washington and Parkinson, 2005). Parameter uncertainty is introduced by choice of constant parameter values to represent variables that vary in time and/or space, or do not correspond to physical quantities. Simulations can be very sensitive to the parameters chosen (Gregory, 2004; Roach et al., 2017). Models may differ in choice and structure of parametrization schemes, contributing to structural uncertainty. Many physical processes are poorly observed and simply cannot be well-represented.

\subsection{Development of sea ice models}

The positive climatic feedback of sea ice in amplifying temperature changes has long been known to climatologists (Brooks, 1926). The sea ice-albedo feedback was included in the first energy balance models of Sellers (1969) and Budyko (1969), by setting the ocean albedo to a high value at low sea surface temperatures to represent sea ice. This is an example of a parametrization with a functional dependence on sea surface temperature. The additional role of sea ice as an insulating shield between the atmosphere and ocean and as a source or sink for freshwater was noted during the development of the very first coupled climate models in the late 1960s (Bryan, 1969). These early models included a simple sea ice component based on large-scale heat balance, with a limited representation of sea ice transport. 
In the 1960s and 1970s, there were major advances in models for sea ice thermodynamics (e.g. Untersteiner 1964, Maykut and Untersteiner 1971, Semtner 1976) and dynamics (e.g. Parmerter and Coon 1972, Coon et al. 1974, Hibler 1979). A dynamicthermodynamic sea ice model (including an ice thickness distribution, rheology and momentum balance) was not coupled to an ocean model until the late 1980s (Hibler and Bryan, 1987). Since then, sea ice models have steadily become more complex. In the following subsections, I summarize some key developments in the various components of sea ice models.

\subsubsection{Ice thickness distribution}

Bulk properties of sea ice depend strongly, and in some cases entirely, on sea ice thickness. For example, the thicker the sea ice, the greater resistance to compression and the larger the magnitude of ice strength. Much more heat is transferred from the ocean to the atmosphere where there is thin or no sea ice: if leads represent $1 \%$ of the ice pack, half of all ocean to atmosphere heat transfer occurs through these leads (Thorndike et al., 1975). Growth and melting of sea ice depend strongly on sea ice thickness, for example through the ice-thickness-ice-growth feedback (Bitz and Roe, 2004). The early models of Bryan (1969) and Bryan et al. (1975) described evolution of a single uniform ice thickness in each grid cell. However, the area covered by a typical global sea ice model grid cell could in reality contain many types of sea ice, ranging from frazil ice on the order of centimetres to large ridges 10 - $20 \mathrm{~m}$ thick.

To capture thickness variations, most current sea ice models divide ice in each grid cell into a finite distribution of thickness categories (Table 3.1). Thorndike et al. (1975) were the first to describe a theory for the ice thickness distribution (ITD). Their ITD arises from two opposing mechanisms: thermodynamic processes acting on the top and bottom of the ice which strive for a single equilibrium ice thickness, and mechanical processes forming leads and pressure ridges, seeking extremes in ice thickness. Generally, dynamics dominates at shorter timescales and thermodynamics at longer timescales. We describe the ITD theory in detail here due to its relevance for the theory of the floe size distribution (see Sec. 2.7 and Chapter 4). 
Consider for each point $\vec{x}$ a region $\mathcal{R}(\vec{x})$ in the ice pack with an area $R$ and a length scale much greater than the typical length scale of floes, ridges and leads. Let $A\left(h_{1}, h_{2}\right)$ be the area within $\mathcal{R}$ covered by ice of some thickness $h$, which lies between two thickness limits $h_{1}$ and $h_{2}$, such that $h_{1} \leq h \leq h_{2}$. The thickness distribution $g(h)$ is then defined by

$$
\int_{h_{1}}^{h_{2}} g(h) \mathrm{d} h=\frac{1}{R} A\left(h_{1}, h_{2}\right),
$$

such that $g(h) \mathrm{d} h$ is the fraction of ocean surface covered by ice with thickness between $h$ and $h+\mathrm{d} h$. The ice thickness $h$ can assume values from zero, where it represents open water, to some upper bound $h_{\max }$. Outside of these limits, $g$ vanishes. Integrating Eqn. 2.1 between these limits gives a conservation equation

$$
\int_{0}^{h_{\max }} g(h) \mathrm{d} h=1 .
$$

When a finite area of ice has a uniform thickness $h_{1}$, in the limit $h_{1} \longrightarrow h_{2}, A\left(h_{1}, h_{2}\right)$ is finite and $g(h)=\delta\left(h-h_{1}\right)$.

It is assumed that every subregion of $\mathcal{R}(\vec{x})$ has nearly the same ITD as $\mathcal{R}(\vec{x})$ itself, so that the function is continuous. A further assumption made is that the density of ice is constant. The detailed velocity of the ice pack is reduced to a smoothed velocity $\vec{v}$ which is near-linear over $\mathcal{R}$, so the ITD has a flux $\vec{v} g$.

Using an Eulerian description in thickness space, the time rate of change of the ITD is then equal to the divergence of its flux plus sources and sinks. The sources and sinks here are thermodynamic processes and mechanical redistribution, $\psi$. Thermodynamic thickening of ice is described by a growth rate function $f$, with units length per unit time. Its magnitude is determined by the flux balance at the top and bottom of the ice, thermal history of ice, distribution of liquid brine inclusions and the thicknesses of ice and snow. Feedbacks between thermodynamic ice changes and the boundary layers are neglected.

To describe the thermodynamic processes, it is useful to define a cumulative thickness distribution:

$$
G(h)=\int_{0}^{h} g\left(h^{\prime}\right) d h^{\prime},
$$

which is the fractional area of ice that is thinner than $h$. If ice changes its thickness 
thermodynamically at a rate $f$, then ice thinner than $h$ at time $t$ will be thinner than $h+f d t$ at time $t+d t$. Hence,

$$
\begin{aligned}
G(h, t) & =G(h+f d t, t+d t) \\
& =G(h, t+d t)+f \frac{\partial G}{\partial h} d t+\mathcal{O}\left(d t^{2}\right) .
\end{aligned}
$$

Differentiating with respect to time,

$$
\frac{\partial G}{\partial t}=-f \frac{\partial G}{\partial h}=-f g
$$

and differentiating with respect to thickness,

$$
\frac{\partial}{\partial h}\left(\frac{\partial G}{\partial t}\right)=\frac{\partial g}{\partial t}=-\frac{\partial}{\partial h}(f g)
$$

Hence the governing ITD equation is:

$$
\frac{\partial g}{\partial t}=-\frac{\partial}{\partial h}(f g)-\nabla \cdot(g \vec{v})+\psi
$$

The terms on the right hand side represent respectively the change in the thickness distribution due to thermodynamic growth/melt, advection of the ice thickness distribution by sea ice dynamics and redistribution of ice between thickness categories due to mechanical processes.

For mechanical redistribution, $g(h)$ is always discretized as a series of Dirac delta functions. For thermodynamic changes in thickness space, $g(h)$ was initially simulated using an Eulerian discretization, where it is assumed that $g(h)$ is distributed uniformly between each category boundary, with the mean thickness of a category always at the midpoint between category boundaries (Hibler, 1980). This method is simple, but diffusive if too few categories are used. Today's models use a discretization for $g(h)$ that is piecewise continuous with linear functions between category boundaries (Lipscomb, 2001). 


\subsubsection{Thermodynamics}

The first global models computed local freezing and melting from the heat balance between the atmosphere and ocean, neglecting the heat capacity and salt content of sea ice (Bryan, 1969). They assumed that snowfall augments thickness, while rain passes directly into the ocean, and allowed local melting to alter ocean salinity (Bryan, 1969). Around the same time, Maykut and Untersteiner (1971) constructed a detailed model of sea ice thermodynamics in one dimension, including the effects of ice salinity, brine pockets trapped within the ice, heat from penetrating shortwave radiation, vertical variations in ice density, conductivity, and specific heat. This was discretized on a vertical grid with $10 \mathrm{~cm}$ spacing and at the time was too computationally expensive to be incorporated into a three-dimensional model. This model was simplified by Semtner (1976) into both a three-layer and a zero-layer version, which assumed a linear temperature profile in the ice and neglected storage of latent and sensible heat. Despite errors in seasonal ice thickness (Semtner, 1976) on the order of $50 \%$, which were shown to have implications for predictions of Arctic change (Semtner, 1984), the zero-layer model was widely adopted and is still used in some models considered for CMIP5 (Table 3.1).

Bitz and Lipscomb (1999) developed an energy-conserving treatment of internal heating and surface melting, including a parametrization for brine pockets, that used multiple internal ice layers. This assumes a fixed salinity profile in the ice and may not be suitable for young first year ice. Some new models (e.g. Hunke et al. 2015) include a formulation which treats sea ice as a mushy layer (Feltham, 2005), i.e. a matrix of pure solid ice bathed in its impurity rich melt (brine), and allows prognostic evolution of salinity.

A range of schemes are used for sea ice albedo. The simplest use fixed values for a limited number of ice types (cold snow, cold ice, melting ice and melting snow, for example) or linearly interpolate between them for different surface temperatures (e.g. Gordon et al. 2000). Intermediate schemes have albedos with dependence on the temperature and thickness of ice and snow and on the spectral distribution of the incoming solar radiation, assuming exponential decay of solar radiation as it penetrates into the ice, for example the Community Climate System Model (CCSM3) parametrization (Briegleb et al., 2004). State-of-the-art multiple-scattering schemes calculate reflected, absorbed and transmitted shortwave radiation for the various ice and snow layers based on prescribed inherent optical properties, for example the Delta-Eddington parametrization (Briegleb and Light, 
2007). The latter case can account for melt ponds, with melt pond area and volume in each ice thickness category evolving according to ice and snow melt, rainfall, and in some cases ice topography (Flocco et al., 2010). Melt pond schemes usually only impact sea ice by their radiative properties, but work is underway to include the impacts of melt pond drainage and re-freezing in sea ice thermodynamics (e.g. Flocco et al. 2015).

Most of the thermodynamics described above can be represented in one dimension. Changes in sea ice also occur laterally, i.e. in two dimensions. To include lateral changes, Parkinson and Washington (1979) introduced a fourth term on the right hand side of Eqn. 2.7 to represent lateral melting using a portion of positive energy input in leads within the ice based on the lead area. Similarly, new frazil ice was permitted to grow in leads in the case of negative energy input. Ebert and Curry (1993) included a similar scheme, which assumed that all solar energy absorbed by the ocean to the depth of the ice thickness was used for lateral ablation, using an empirical form for the absorption of solar energy suggested by Maykut and Perovich (1987). This was thought to overestimate lateral melt. The fraction of available ocean heat used for lateral melting should depend on floe size and geometry, variables which are not explicitly simulated in large-scale sea ice models. A common approach today is to assume a single fixed sea ice floe size to calculate the sea ice perimeter and assume a uniform melt rate around floe edges following Steele (1992) (Table 3.1). In models without an explicit lateral melt term, it is assumed that lateral melting is accounted for implicitly by the bottom melting of thin ice (Bitz et al., 2001; Vancoppenolle et al., 2009).

\subsubsection{Dynamics}

In the early model of Bryan (1969), ice was advected in-step with ocean currents, together with some lateral diffusion, and it was assumed that all motion ceases when the ice reaches some critical thickness. This approach is still used in some models (e.g. HadCM3, Gordon et al. 2000). However, most of today's sea ice models include equations for sea ice momentum, a constitutive law that describes the material properties of the ice (rheology), transport, and mechanical deformation. 
Hibler (1979) proposed a momentum balance equation to calculate ice velocity:

$$
m \frac{\mathrm{D} \vec{u}}{\mathrm{D} t}=-m f \vec{k} \times \vec{u}+\overrightarrow{\tau_{a}}+\overrightarrow{\tau_{w}}-m \hat{g} \nabla H+\vec{F}
$$

Here, $\vec{k}$ is a unit vector normal to the surface, $\vec{u}$ is the ice velocity, $f$ is the Coriolis parameter, $m$ is the ice mass per unit area, $\overrightarrow{\tau_{a}}$ and $\overrightarrow{\tau_{w}}$ are the forces due to air and water stresses, $H$ is the sea surface height and $\vec{F}$ is the force due to internal stress. $F_{i}=$ $\partial \sigma_{i j} / \partial x_{j}$ is calculated from some constitutive law,

$$
\sigma_{i j}=\sigma_{i j}\left(\epsilon_{i j} P\right)
$$

where $\sigma_{i j}$ is the two-dimensional stress tensor, $\epsilon_{i j}=\frac{1}{2}\left(\frac{\partial u_{i}}{\partial x_{j}}+\frac{\partial u_{j}}{\partial x_{i}}\right)$ is the strain rate tensor and $P$ is the ice strength. The constitutive law describes the rheology of the material (how it flows and deforms), and relates the stress tensor to the ice velocity.

Different rheologies for sea ice have been suggested based on field campaigns, notably the Arctic Ice Dynamics Joint Experiment (AIDJEX) in the early 1970s. Coon (1972) was the first to suggest that pack sea ice could be described as a plastic material, i.e. after some critical stress is applied, irreversible deformations are induced. Coon et al. (1974) modelled plastic behaviour and allowed elasticity for certain strain states, but this required substantial mathematical complexity. Hibler (1979) described a simpler approach of treating sea ice as a non-linear viscous compressible material, which acts as a plastic at normal deformation rates and as a linear viscous fluid which 'creeps' for very small deformation rates. This viscous-plastic (VP) approach has limited time-step stability, and was not implemented in climate models for some time. A parametrization of sea ice dynamics as a cavitating fluid (CF) where the ice pack is assumed to have no shear strength (Flato and Hibler, 1992) was widely adopted instead. The introduction of an artificial time-dependent elastic term into the viscous plastic law (EVP) (Hunke and Dukowicz, 1997) improved numerical efficiency and a number of current models use this scheme (Table 3.1). Although the VP scheme improved simulation of sea ice dynamics (Kreyscher et al., 2000), concerns over accuracy have been raised (Gray, 1999; Coon et al., 2007; Rampal et al., 2008) and alternative rheological schemes are an active area of research (Tsamados et al., 2013; Dansereau et al., 2016). Note that both the VP and CF approaches assume that the mixture of sea ice floes and leads can be treated as a continuum. 
The ice strength $P$ has been described using an empirical function that is linear in thickness and exponential in concentration, allowing ice to strengthen as it thickens, and capturing the weakness of low-concentration ice and the strength of high-concentration ice (Hibler, 1979). Rothrock (1975) described an alternative scheme where the ice strength is related to the work done during mechanical deformation (ridging). They assumed that the work done in deforming the material is equal to the energy sinks during ridging, namely potential energy production and frictional energy loss.

Rothrock (1975) proposed a form for mechanical redistribution, $\psi$, by considering divergence (where open water is exposed), convergence (where open water closes up and thin ice may be rearranged into ridges or piles) and shear (where force along some cracks leads to open water creation and pressure ridges). Other mechanisms, such as thermal cracking or uneven hydrostatic loading, are neglected. $\psi$ is a function of the strain rate tensor, a physical quantity that describes the rate of change of the deformation of a material. Assuming that ice has no directional properties (it is isotropic), $\psi$ depends only on two invariants of the strain rate tensor.

The form for $\psi$ is constructed subject to the following constraints. Integrating Eqn. 2.7 subject to the ITD conservation equation shows that mechanical redistribution compensates any gain or loss in area. Unlike thermodynamics, mechanical redistribution does not change volume per unit area, i.e. $\int_{0}^{h_{\max }} h \psi d h=0$. In the case of divergence, it is assumed that open water is exposed to make up for the ice area exported from the region. In the case of convergence, thin ice is converted into thicker ice to make room for the flux of ice into the region. We must (a) specify the ITD of ice participating in ridging, (b) specify a rule to transform thin to thick ice while conserving volume, and (c) calculate the ITD of newly ridged ice. Simple rules for this were proposed by Hibler (1980) and Thorndike et al. (1975), but more complex schemes have been used since then (e.g. Lipscomb et al. 2007). In the case of pure shear, some component normal to the crack will cause local deformation and redistribution, using the same ridging and opening modes.

Given the momentum equation, constitutive law and ice strength, the ice velocity field can be calculated. Then, the sea ice must be advected whilst conserving mass and energy, requiring continuity equations. For the ice area fraction in thickness category $n, a_{i n}$, this 
is

$$
\frac{\partial}{\partial t} a_{i n}+\nabla \cdot\left(\mathbf{u} a_{i n}\right)=0
$$

with similar forms for ice volume, snow volume, ice energy, snow energy and area- or volume-conserving tracers. A variety of numerical schemes are used to solve these transport equations.

\subsection{CICE}

We use the Los Alamos sea ice model CICE5.1 (Hunke et al., 2015) in Chapters 3, 4 and 6. It is also used within a number of state-of-the-art global climate models, including the latest Hadley Centre models HadGEM2 and HadGEM3, and the Community Earth System Model CCSM4. CICE contains a comprehensive description of sea ice processes, which is arguably the best available for use within GCMs. Different physics can be selected by the user; for example it can be run using different rheologies, and with a fixed salinity profile, or the mushy layer scheme. This section describes the main physical equations of the model and the settings used in the experiments described in this thesis.

Whether run as part of a coupled climate model or in stand-alone mode, CICE requires atmospheric and oceanic forcing input through a flux coupler. The atmospheric forcing data include wind velocity, specific humidity, air density, air potential temperature and air temperature at a given atmosphere level height, as well as shortwave and longwave radiation, and snowfall and rainfall rates. The oceanic forcing data include the freezing/melting potential, sea surface temperature, sea surface slope and surface ocean currents.

The principal equation used in CICE is Eqn. 2.7 as proposed by Thorndike et al. (1975). This is solved by partitioning the ice pack into $N_{c}$ discrete categories for ice thickness plus one for open water. Bitz et al. (2001) showed that $N_{c}=5$ was sufficient to capture most large-scale behaviour. Two of the terms on the right hand side are set to zero in turn, and then each transport equation is solved separately.

The $N_{c}$ thickness categories consist of $N_{i}$ ice layers and $N_{s}$ snow layers. Model defaults are $N_{i}=4$ and $N_{s}=1$, with thicknesses $\Delta h_{i}=h_{i} / 4$ and $\Delta h_{s}$ respectively. The enthalpy $q$ is the negative of the energy required to melt a unit volume of ice or snow and 
raise its temperature to $0^{\circ} \mathrm{C}$.

Balancing fluxes at the top and bottom interfaces (with all fluxes positive downwards), the net surface energy flux from the atmosphere to the ice, $F_{o}$, is,

$$
F_{o}=F_{s}+F_{l}+F_{L \downarrow}+F_{L \uparrow}+(1-\alpha)\left(1-i_{o}\right) F_{S W}
$$

The first and second terms on the right hand side are the sensible heat flux $F_{s}=C_{s}\left(\Theta_{a}-\right.$ $\left.T_{\text {sfc }}\right)$ and the latent heat flux $F_{l}=C_{l}\left(Q_{a}-Q_{\text {sfc }}\right)$. Here, $C_{s}$ and $C_{l}$ are non-linear turbulent heat transfer coefficients, which are calculated from drag coefficients in the atmospheric boundary layer, $\Theta_{a}$ and $Q_{a}$ are the air potential temperature and air specific humidity, and the surface humidity is $Q_{\mathrm{sfc}}=q_{1} \exp ^{-q_{2} / T_{\text {scc }}} / \rho_{a}$, where $q_{1}$ and $q_{2}$ are constants, and $\rho_{a}$ is air density. The third and fourth terms are the incoming longwave radiation, $F_{L \downarrow}$, and the emitted longwave radiation $F_{L \uparrow}=\epsilon \sigma T_{\text {sfc }}^{4}$, where the constants $\epsilon$ and $\sigma$ are emissivity and the Stefan-Boltzmann constant. The fifth term on the right hand side is absorbed shortwave radiation, where $\alpha$ is the shortwave albedo, and $i_{o}$ is the fraction of absorbed shortwave flux that penetrates into the ice, both calculated from the albedo parametrization, and incoming shortwave $F_{S W}$ is provided by the forcing.

We use the CCSM3 parametrization for surface albedo (see above). Shortwave radiation is attenuated through the ice according to Beer's Law,

$$
I(z)=I_{o} \exp ^{-k_{i} z}
$$

where $I(z)$ is the shortwave flux that reaches depth $z$ beneath the surface without being absorbed, and $k_{i}$ is the bulk extinction coefficient for solar radiation in ice, set to a constant value for visible wavelengths. A fraction $F_{S W \downarrow \downarrow}$ passes into the ocean.

The heat flux from the ice into the ocean, $F_{\text {bot }}$ is given by

$$
F_{\mathrm{bot}}=-\rho_{w} c_{w} c_{h} u_{*}\left(T_{w}-T_{f}\right)
$$

where the constants $\rho_{w}$ and $c_{w}$ are the density and heat capacity of sea water, $c_{h}=0.006$ is the heat transfer coefficient, $u_{*}=\sqrt{\left|\overrightarrow{\tau_{w}}\right| \rho_{w}}$ is the friction velocity, and $T_{w}$ and $T_{f}$ are the sea surface temperature and salinity-dependent freezing temperature, respectively. 
Then, given the ice and snow thickness, temperature and enthalpy as well as the surface forcing at the interfaces at time $m$, the thermodynamics model advances the temperature to $m+1$ by solving a set of equations for the new temperatures and computing any melting or growth. Each temperature is coupled to the temperatures of the layers immediately above and below it by finite difference heat conduction equations. This matrix equation is solved to obtain new temperatures at time $m+1$, given temperatures $T_{\text {sfc }}, T_{s}$ and $T_{i, k}$ at time $m$. These equations can be formulated using one of three thermodynamics schemes. The experiments conducted for this thesis use the scheme described in Bitz and Lipscomb (1999), which assumes a fixed ice salinity profile.

In the ocean, frazil ice can form in the thinnest ice category (see Sec. 4.3 for further details). At the top surface of the ice, ice can melt via conduction if $T_{\mathrm{sfc}} \geq 0$. If $T_{\mathrm{sfc}}<0$, it is required that $F_{o}=F_{c t}$. Hence

$$
q \delta h=\left(F_{o}-F_{c t}\right) \Delta t \text { if } F_{o}>F_{c t} \text { else } q \delta h=0 .
$$

$F_{c t}$ is the conductive heat flux from the surface to the ice interior. If the layer melts completely, $F_{c t}$ can begin to melt the next layer; if the bottom layer melts completely, energy is added to the ocean mixed layer.

Ice cannot grow at the top surface by conduction. However, snow-ice can form here. If snow falls and piles up below sea level, it is replaced by the same level of sea ice. Snow or ice can sublimate and vapour can form snow or ice via the latent heat flux, according to

$$
\delta h=\frac{F_{l} \Delta t}{\rho L_{v}-a} .
$$

At the bottom surface of the ice, both growth and melting can occur via imbalance of the ice-ocean heat flux (Eqn. 2.13) and conductive heat flux at the bottom surface, $F_{c b}=$ $K_{i, N+1}\left(T_{i N}-T_{f}\right) / \Delta h_{i}$. The growth or melting is determined by

$$
q \delta h=\left(F_{c b}-F_{b o t}\right) \Delta t
$$

At the ice edge, a fraction of ice is melted laterally following Steele (1992), assuming that melting occurs uniformly at a rate $w_{l a t}$ around the perimeter of each floe, with the total perimeter calculated by assuming a fixed floe size of $L=300 \mathrm{~m}$. See Subsec. 3.3.4 for a 
derivation of the change in concentration due to lateral melt.

The dynamics scheme uses Eqn. 2.8, with internal stresses calculated from the EVP constitutive law. The ice strength is computed at the start of each timestep from the ridging parametrization and is then held fixed. The momentum and stress equations are subcycled around 120 times, updating the stresses, viscosities and velocities. The scheme for mechanical redistribution follows Thorndike et al. (1975) with the improvements suggested by Lipscomb et al. (2007). CICE uses a 2-D incremental remapping scheme for the transport of basic state variables (Lipscomb and Hunke, 2004), with which transport of additional tracers is computationally cheap (Hunke et al., 2010).

\subsection{Sea ice floe size distribution}

As introduced in Chapter 1, sea ice is made up of individual floes which vary widely in horizontal size. The size of floes may be important for the polar climate system. Below I review relevant literature regarding observations and models of the sea ice floe size distribution.

\subsubsection{Definition of the floe size distribution}

The concept of a floe size distribution (FSD) was first described by Rothrock and Thorndike (1984). Supposing $P$ is some constant property of a sea ice floe relating to its size, they define a fractional area $F$ in some region $\mathcal{R}$ (with area $R$ ) covered by floes for which $P$ is not less than some constant value $p$ as

$$
F(p, \mathcal{R})=\frac{1}{R} \iint_{\mathcal{R}} H[P(x, y)-p] \mathrm{d} x \mathrm{~d} y, x, y \in \mathcal{R}
$$

Here, the Heaviside function $H(q)=1$ if $q \geq 0$ and $H(q)=0$ if $q<0$. As $p$ tends to zero, $F\left(0^{+}, R\right)$ is the ice concentration, while $F(0, R)=1$.

The definition accounts for floes which overlap the boundary of $\mathcal{R}$, for which only the area of the floe within $\mathcal{R}$ is included, but the value of $P$ is for the whole floe. The property $P$ could be floe area, some representative floe length scale, or perimeter; their observations suggest that these properties are highly correlated so any could be used in 
the distribution.

Besides the cumulative areal distribution $F$, an alternative description is the cumulative number density $N(p, \mathcal{R})$, the number of floes per unit area in a region $\mathcal{R}$ for which $P$ is no less than $p$. The non-cumulative probability density function, $n(p) \mathrm{d} p$, which is the fractional number of floes with size between $p$ and $p+\mathrm{d} p$, is another alternative. Differences between cumulative and non-cumulative distributions are discussed further in Stern et al. (2018a).

\subsubsection{Observations of floe sizes}

Unlike sea ice concentration, sea ice floe sizes cannot be obtained from passive microwave radiometers. They can be estimated from images captured by cameras carried by aerial vehicles, visible-band satellites (e.g. Landsat, MODIS, MEDEA) and synthetic aperture radar (SAR, e.g. TerrSAR-X, RADARSAT-2) using image processing tools to separate individual floes (e.g. Fig. 2.3). Table 2.1 shows a selection of observational studies, including image types, region, dates and total areal coverage. The spatial and temporal coverage of observations is limited (Figs. 2.4 and 2.5), and the range of floe sizes resolved varies between studies (Table 2.1). Naturally, observational uncertainty is introduced by (and not limited to) (1) lack of distinct floe edges, (2) floes at sizes below the image resolution, (3) floes extending beyond the image frame, and (4) visual interference such as cloud cover in visible-band imagery. Some studies use similar methods (Stern et al., 2018a), but a comprehensive intercomparison of image processing methods for floes has not yet been carried out.

Nearly all studies fit power laws to either the cumulative or non-cumulative floe number density distribution, i.e. for the non-cumulative distribution $n(p)=c p^{-\alpha}$ for some normalization constant $c$ and an exponent $\alpha>0$. The equivalent cumulative distribution would be $N(p)=C p^{-\alpha+1}$ for normalization constant $C=c /(\alpha-1)$. In most cases (see Table 2.1), the value of the exponent is obtained from a least squares regression on a loglog plot, which may lead to biased estimates of the exponent, and depends on the binning of the data (Clauset et al., 2009). Only two studies in Table 2.1 conduct goodness-of-fit tests to determine whether the data are power-law distributed (Hwang et al., 2017; Stern et al., 2018b). Although power law exponents are reported, the data do not universally 
show straight lines in log-log space (e.g. Fig. 2.6 below reproduced from Wang et al. 2016; Steer et al. 2008; see also discussion in Herman 2010). A limited amount of floe size data (Hartmann et al., 1992; Kottmeier et al., 1994) has also been fit to a function of sea ice concentration (Lüpkes et al., 2012), but little information on this data is accessible so it is not shown in Table 2.1.
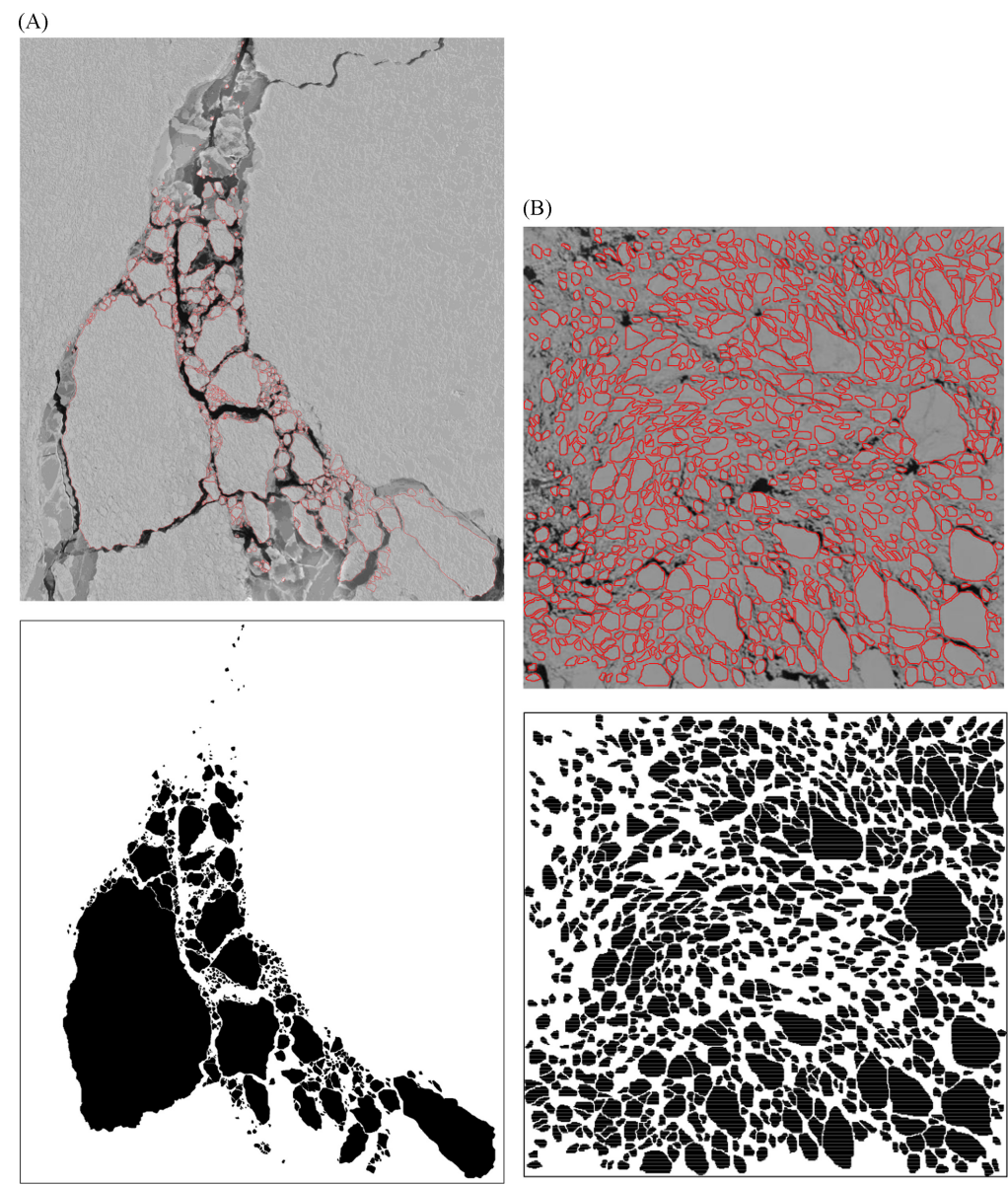

Figure 2.3: An example showing the process to extract ice floes from (A) a camera photo. Image taken from the helicopter at 5:14 on November 5 and (B) MODIS image on September 24. For each case, upper figure shows original video image with each ice floe outlined in red after the process of determining ice edges; and lower figure shows extracted floes to be measured. For (A) the area is $1933 \mathrm{~m}$ x $2254 \mathrm{~m}$ and 630 ice floes are included for analysis. For (B) the area is $131 \mathrm{~km}$ x $126 \mathrm{~km}$ and 838 ice floes are included for analysis. Figure and caption reproduced from Toyota et al. (2011). 


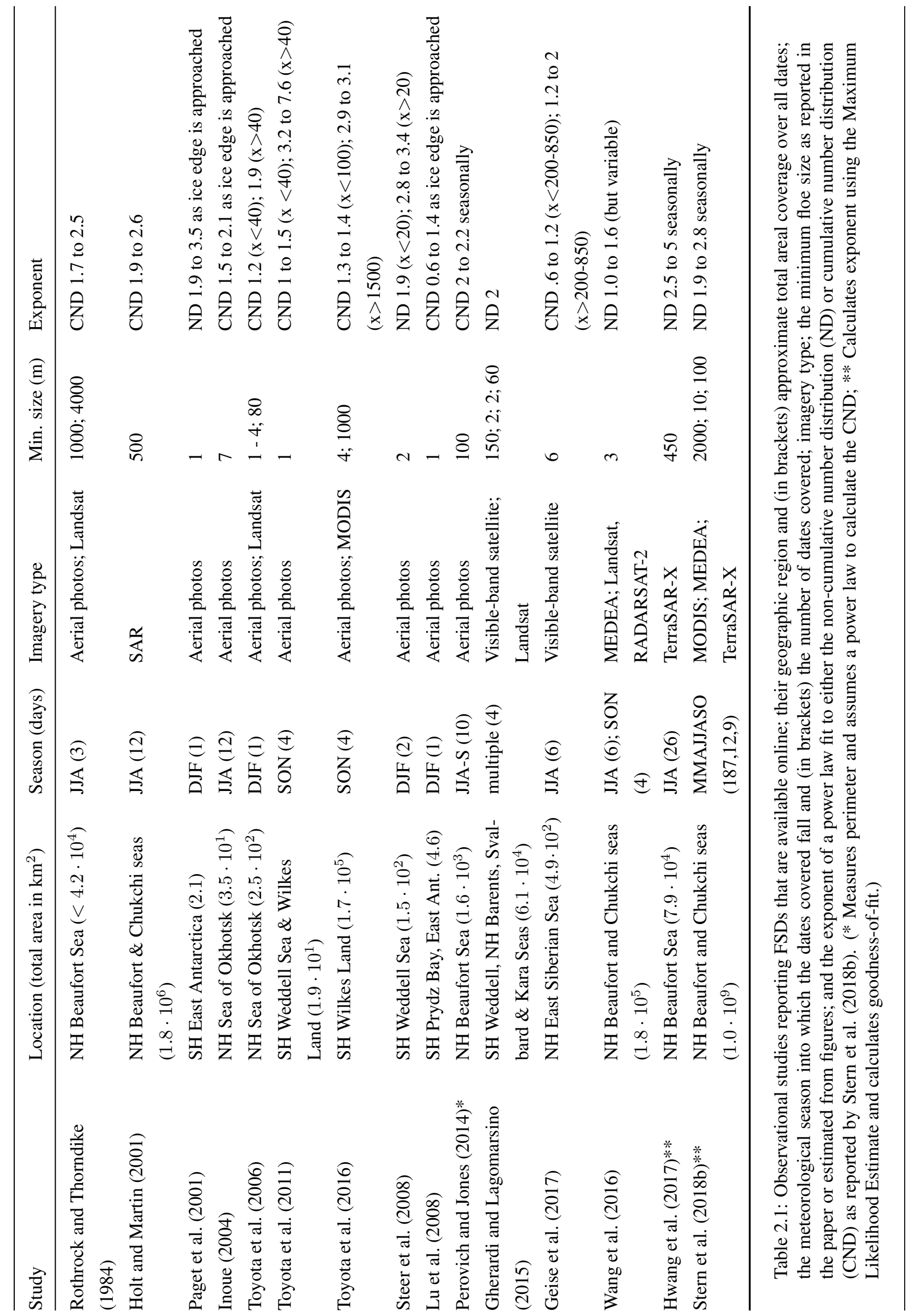




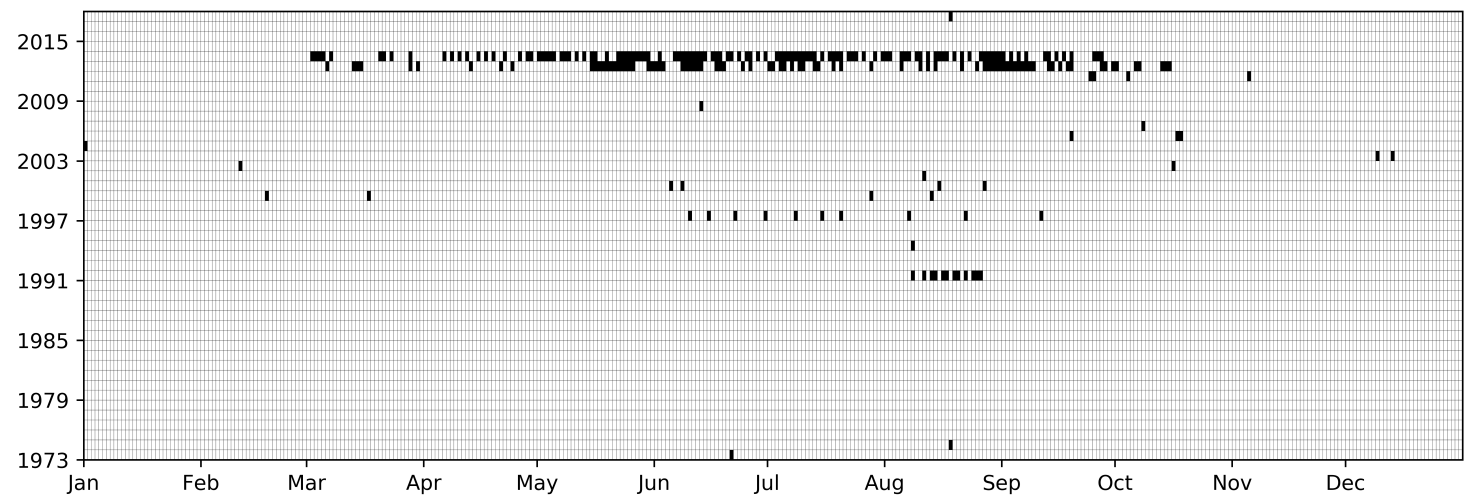

Figure 2.4: Temporal coverage of studies listed in Table 2.1. Black squares denote dates for which any study reports any floe size data at any resolution. The higher coverage in 2013 and 2014 is from MODIS data in Stern et al. (2018a), which only resolves floes greater than $2 \mathrm{~km}$ in diameter.

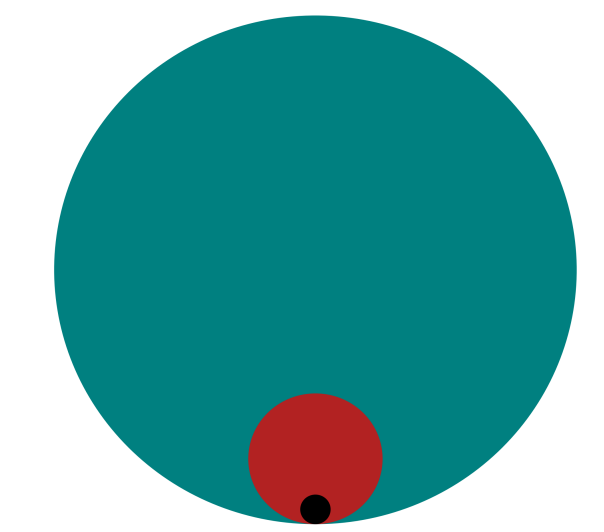

Combined $\mathrm{NH} \& \mathrm{SH}$ maximum SIE

Areal coverage of studies resolving floes $<1 \mathrm{~km}$

Areal coverage of studies resolving floes $<300 \mathrm{~m}$

Figure 2.5: The total spatial coverage (summed over all dates) of imagery from studies listed in Table 2.1 that (red) resolves floe sizes below $1 \mathrm{~km}$ and (black) resolves floe sizes below $300 \mathrm{~m}$ in diameter, relative to (blue) the sum of the Arctic and Antarctic approximate average maximum sea ice extents (15 and 18 million $\mathrm{km}^{2}$ respectively, totalling 33 million $\mathrm{km}^{2}$ ).

An often-reported phenomenon in the cumulative number distribution is a 'transition,' or point-of-inflexion, such that the slope of the distribution differs between two floe size regimes (Toyota et al., 2006, 2011, 2016; Geise et al., 2017). This is sometimes referred to as a 'split power law' (Bennetts et al., 2017). It has also been reported in the noncumulative number distribution (Steer et al., 2008). Toyota et al. $(2006,2011)$ hypothesized that this transition occurs at the minimum floe size that can be fractured by flexural strain imposed on the ice by ocean surface waves (Mellor, 1986) and therefore does not appear in the FSD for the ice interior (Toyota et al., 2016). Geise et al. (2017) also argued 


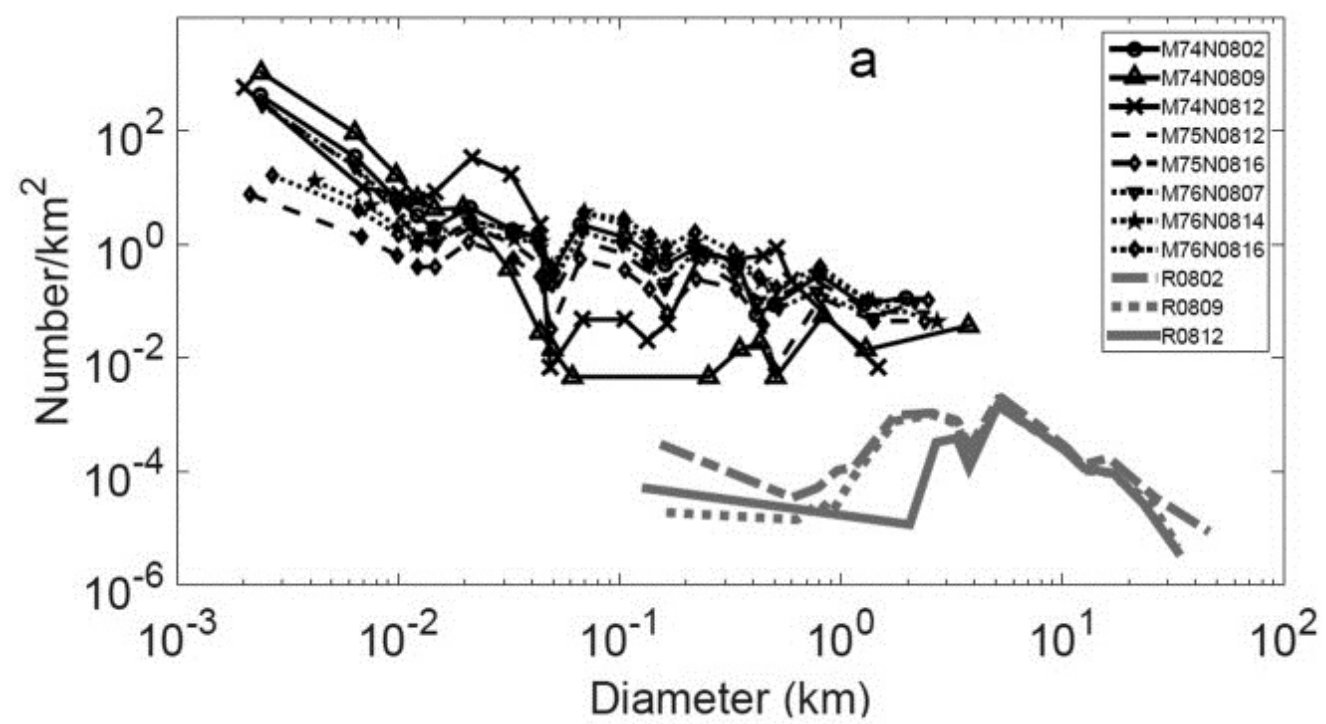

Figure 2.6: The floe size number density estimated from MEDEA images (visible-band, resolution of $1 \mathrm{~m}$ ) in black and RADARSAT-2 images (synthetic aperture radar, resolution of $100 \mathrm{~m}$ ) in grey, in the Beaufort and Chukchi Seas in August 2014. Figure 13a reproduced from Wang et al. (2016).

that the transition arose from wave fracture, although they were unable to reproduce it in the fracture model they proposed.

However, this behaviour can also be interpreted as gradual bending rather than a sharp transition (Herman, 2010), and — as discussed at length by Stern et al. (2018b) — may arise simply due to a truncation effect. If a non-cumulative distribution is a power law, then the equivalent cumulative distribution defined on a finite size range (i.e. an upper-truncated power law) shows concave-down curvature (Stern et al., 2018b). Any under-sampling of large floes, for example due to the limited size of the domain, may also contribute to the bending of curves for large sizes (Wang et al., 2016; Stern et al., 2018b). It is not clear whether the reported 'split power law' arises from mathematical considerations, undersampling or the underlying physics governing floe sizes.

A re-analysis of all data reported by studies included in Table 2.1-which calculates exponents appropriately, tests goodness-of-fit, excludes the range of sizes under-sampled and uses a non-cumulative distribution to avoid truncation effects - is needed. Regrettably, much of the data is not publicly available. Moreover, the published studies cover a handful of dates and small regions (Figs. 2.4 and 2.5), despite the large inter-annual, seasonal and spatial variability in floe size (e.g. Rothrock and Thorndike 1984; Perovich and Jones 2014). This limits our understanding of the inherent properties of the FSD, as 
well as how it evolves.

\subsubsection{Processes driving floe sizes}

Observational studies have speculated how different physical processes drive variability in floe sizes, with most focusing on the role of ocean surface waves. Besides the change in power law exponent with floe size (Toyota et al., 2006; Steer et al., 2008; Toyota et al., 2011, 2016; Geise et al., 2017), the power law exponent has also been observed to vary with distance from the ice edge (Paget et al., 2001; Inoue, 2004). The location of a marked change in the exponent may correspond to the furthest distance that penetrating ocean surface waves are able to fracture ice (Paget et al., 2001; Inoue, 2004). Wave fracture occurs in discrete events (Perovich and Jones, 2014), such as storms, which result in a weak trend towards either smaller floes or a greater number of floes (Holt and Martin, 2001). As well as fracturing ice, the wave field also places a limit on the size of new floes forming during freezing conditions (Shen et al., 2001; Shen, 2004). Other processes that may play a role in determining floe sizes include lateral melt (e.g. Perovich and Jones 2014), the freezing together of floes (e.g. Toyota et al. 2006), floe collisions (e.g. Lu et al. 2008), and the melting apart of floes along ridges or other weak points (e.g. Steer et al. 2008, Arntsen et al. 2015).

\subsubsection{Importance of floe sizes}

The sizes of floes are thought to have wider importance for the climate system due to their role in determining the amount of melt occurring on the sides of floes (Steele, 1992). Lateral melt depends on the perimeter of floes per unit area, so a region of small floes would melt faster than a region of large floes (Steele, 1992; Arntsen et al., 2015). The lateral melt rate is very sensitive to the distribution of floe sizes (Toyota et al., 2006).

Lateral melt expands the area of open water, lowering surface albedo and allowing heat transfer between the atmosphere and ocean (e.g. Asplin et al. 2012), and could cause greater open water expansion than an equivalent amount of bottom or surface melt. This increases solar heat input to the upper ocean-an important factor in Arctic sea ice decay (Maykut and Perovich, 1987; Maykut and McPhee, 1995) and possibly the dominant fac- 
tor in Antarctic sea ice decay (Nihashi and Ohshima, 2001) — and may result in a positive feedback (Asplin et al., 2014; Perovich et al., 2008). Fractured ice may also allow waves to penetrate further into the ice pack, another positive feedback, increasing susceptibility to lateral melt (Asplin et al., 2014). The lateral melt feedback has been implicated in rapid ice loss events in the Arctic (e.g. Perovich et al. 2008; Asplin et al. 2014) and in regional variability of Antarctic sea ice trends (Kohout et al., 2014).

Besides lateral melt, the FSD plays a role in other physical processes relevant to the polar climate system. The sizes of floes determine the rheological behaviour of sea ice, with small and spaced-out floes behaving like a viscous material with collisional interactions, and closely-packed large floes like a plastic material (Shen et al., 1987; Feltham, 2005; De Silva et al., 2015). The distribution of floe edges affects surface roughness (Steele et al., 1989; Lüpkes et al., 2012) and may be important for local sea ice drift and atmospheric fluxes (Lüpkes et al., 2012). Ocean eddies may develop at floe edges due to melting and freezing around floes (Smith, 2002; Horvat et al., 2016). The spatial distribution of floes has some relation to the spatial distribution of leads, the dominant control on turbulent heat transfer between the ocean and the atmosphere in the Arctic (Marcq and Weiss, 2012). Floe size also impacts the evolution of wave spectral properties within sea ice cover (Montiel et al., 2016).

\subsubsection{Modelling floe size}

In contrast to the large number of observational studies conducted during the decades following Rothrock and Thorndike (1984), climate model implementations of FSD were not considered until very recently. Discrete-element models consider individual floes (e.g. Herman 2016) but at present only simulate two dimensions. If CMIP5 models include representation of floe size at all, it is in the form of a single fixed floe diameter used only to calculate lateral melt (e.g. Steele 1992).

Lüpkes et al. (2012) proposed a functional dependence of average floe diameter on sea ice concentration based on observations by Hartmann et al. (1992) and Kottmeier et al. (1994) with large uncertainties (Lüpkes et al., 2012). This form for floe diameter has been

implemented in CICE for the calculation of form drag (Tsamados et al., 2014) and lateral melt (Tsamados et al., 2015), but has limited observational justification. Areas of high sea ice concentration may consist of closely-packed small floes (e.g. Fig. 2.1b and f). 
Variation in floe size below the grid resolution for large-scale models can be large and the sub-grid-scale distribution of sizes is important for determining lateral melt (Toyota et al., 2006). Moreover, given that observations exhibit some power-law-like behaviour, the FSD will not be well-characterized by an average size (Clauset et al., 2009).

Dumont et al. (2011) and Williams et al. (2013a,b) considered temporal evolution of the FSD due to fracture by ocean surface waves, but constrained the FSD to fit a split power law distribution following Toyota et al. (2011). Besides the concerns discussed by Stern et al. (2018b) and summarized above, this approach does not allow the FSD to freely evolve under different physical processes. Following these studies, and almost concurrently, Zhang et al. (2015) and Horvat and Tziperman (2015) described evolution of a sub-grid-scale floe size distribution due to thermodynamic and dynamic processes, presenting results from single-column models. A strong advantage of the Horvat and Tziperman (2015) approach in comparison to the Zhang et al. (2015) approach is the definition of a joint floe size and ice thickness distribution (FSTD), as well as the descriptions of processes affecting the FSTD based on underlying physics. At the beginning of this $\mathrm{PhD}$ project, neither approach had been implemented in a large-scale sea ice model.

During this $\mathrm{PhD}$, Zhang et al. (2016) demonstrated results from the Zhang et al. (2015) approach in an Arctic-wide model. Bennetts et al. (2017) presented an alternative model for evolution of floe sizes that had no sub-grid-scale variation. Other groups have begun work on the topic, which is increasingly attracting attention in the sea ice modelling community. This may have been sparked by recent studies alluding to the importance of the FSD (Perovich et al., 2008; Asplin et al., 2014; Kohout et al., 2014; Arntsen et al., 2015). The present study advances understanding of the sea ice floe size distribution to aid this emerging field of research. 


\section{Chapter 3}

\section{Consistent biases in Antarctic sea ice concentration simulated by climate models}

This chapter, published as Roach et al. (2018a), presents an assessment of Antarctic sea ice simulated by current climate models relative to satellite observations. Simulation of Antarctic sea ice is generally poorer than Arctic sea ice, but few studies have comprehensively assessed it. Previous model evaluations have focused on sea ice extent; in contrast, this work uses alternative metrics that account for sea ice concentration values and the regional distribution of sea ice. We find that models show consistent biases in sea ice concentration relative to observations, and that these can be partially explained by model representation of lateral melt. We carry out numerical experiments with a coupled ocean-sea ice model that indicate that representation of sea ice concentration is sensitive to the constant floe size parameter used to calculate lateral melt. This motivates prognostic simulation of sea ice floe size, which is investigated in Chapter 4.

\subsection{Abstract}

The simulation of Antarctic sea ice in global climate models often does not agree with observations. In this study, we examine the compactness of sea ice, as well as the regional distribution of sea ice concentration, in climate models from the latest Coupled Model Intercomparison Project (CMIP5) and in satellite observations. We find substantial differences in concentration values between different sets of satellite observations, particu- 
larly at high concentrations, requiring careful treatment when comparing to models. As a fraction of total sea ice extent, models simulate too much loose, low-concentration sea ice cover throughout the year, and too little compact, high-concentration cover in the summer. In spite of the differences in physics between models, these tendencies are broadly consistent across the population of 40 CMIP5 simulations, a result not previously highlighted. Separating models with and without an explicit lateral melt term, we find that inclusion of lateral melt may address the over-estimation of low-concentration cover. Targeted model experiments with a coupled ocean-sea ice model show that choice of constant floe diameter in the lateral melt scheme can also impact representation of loose ice. This suggests that current sea ice thermodynamics contribute to the inadequate simulation of the lowconcentration regime in many models.

\subsection{Introduction}

The cycle of sea ice growth and melt in the Southern Ocean is one of the largest seasonal signals on Earth. The heterogeneity of the sea ice cover and distribution of open water areas determine regional albedo, the reflectivity of the Earth's surface. This in turn impacts entrainment of irradiative energy into the ocean mixed layer (Asplin et al., 2014) and the atmospheric energy budget (Previdi et al., 2015). Sea ice production, which increases salinity, in areas of open water strongly impacts the rate of Antarctic Bottom Water formation (Goosse et al., 1997), the deepest water mass. Regional sea ice concentration thus plays an important role in the coupled climate system.

Coupled climate model output collated by the World Climate Research Programme (WCRP) under the Coupled Model Intercomparison Project (CMIP) protocol are a valuable resource for understanding Earth's climate system. Over 20 groups worldwide have contributed simulations to the latest project (CMIP5) from their models, many of which are developed independently and include different physics. The sea ice components of these models range in complexity, from single-layer, ocean-advected, limited-rheology models (e.g. HadCM3; Gordon et al., 2000) to multi-layer, multiple thickness category models with a non-linear viscous plastic rheology and explicit melt pond formation (e.g. NorESM; Bentsen et al., 2013; Hunke et al., 2015). Advances in Earth system modelling have somewhat improved simulation of Arctic sea ice compared to the previous intercomparison project (CMIP3) (Stroeve et al., 2012), although this may reflect changes in 
forcings (Rosenblum and Eisenman, 2016) or tuning strategy (Notz, 2015) rather than changes in model physics. Simulation of Antarctic sea ice is not considered to have improved (Mahlstein et al., 2013).

To make assessments like these, most model evaluation studies quantify agreement between sea ice models and observations using sea ice extent, which is simply the area of all grid cells with more than $15 \%$ sea ice concentration. Turner et al. (2013) find a wide range of seasonal cycles and trends in Antarctic sea ice extent across the CMIP5 ensemble. Compared to observations, they find that a majority of models underestimate the minimum sea ice extent in February. Shu et al. (2015) evaluate simulated sea ice volume and thickness as well as sea ice extent, finding that the CMIP5 multi-model ensemble mean sea ice extent is fairly well simulated, though worse in the Antarctic than in the Arctic, but suggest that the sea ice cover is generally too thin. Zunz et al. (2013) find that all models overestimate inter-annual variability of Antarctic sea ice extent, particularly in winter. They conclude that no CMIP5 model produces Antarctic sea ice in reasonable agreement with observations over the satellite era.

Using only sea ice extent means that these model evaluation studies do not take into account any sub-grid-scale sea ice information, or the regional distribution of sea ice. As discussed by Notz (2014) and Ivanova et al. (2016), model simulations with the same sea ice extent could have very different sea ice cover characteristics. Notz (2014) instead examines the frequency distribution of summer Arctic sea ice concentration, finding that around half the CMIP5 models have a 'compact' ice cover ( $>0.4$ of grid cells with more than $90 \%$ sea ice concentration) and the rest have a 'loose' ice cover. Ivanova et al. (2016) present a similar analysis for the Antarctic, but show only the CMIP5 multi-model mean and do not discuss the results in detail, focusing instead on the alternative metrics they developed.

In this study we examine model agreement with observations using various simple metrics that account for sea ice concentration values and the regional distribution of sea ice. Our aim is to identify biases in Antarctic sea ice that are common across multiple models. We then carry out targeted model experiments to investigate the role of sea ice model thermodynamics in these biases. 


\subsection{Methods}

\subsubsection{CMIP5 Models}

A series of experiments from different global climate models were carried out for the Coupled Model Intercomparison Project, Phase 5 (CMIP5; Taylor et al. 2012). Output is freely available online from the Program for Climate Model Diagnosis and Intercomparison. The historical experiments, which are forced by observed natural and anthropogenic forcings, end in 2005. To obtain a more contemporary overview, we also consider the first nine years of projection experiments from the mid-range mitigation emission scenario (RCP4.5). Due to the availability of observations (see below), we conduct analysis using 1992-2014. We select the first ensemble member for all models that provide monthly sea ice concentration for both the historical and RCP4.5 experiments, resulting in a set of 40 models (see Table 3.1).

\subsubsection{Observations}

Passive microwave radiometers deployed on satellites measure the brightness temperature of the Earth's surface, and can be used to infer sea ice concentration. There can be large differences between satellite observations (Bunzel et al., 2016), as various observational data sets apply different algorithms to convert passive-microwave signals into sea ice concentration. As summarized by Ivanova et al. (2014), differences between algorithms are caused by (1) choice of radiometer channels; (2) tie-points, which are the brightness temperatures used to identify different surfaces; (3) sensitivities to changes in physical temperature of the surface; and (4) weather filters, which correct for atmospheric effects falsely indicating the presence of sea ice. 


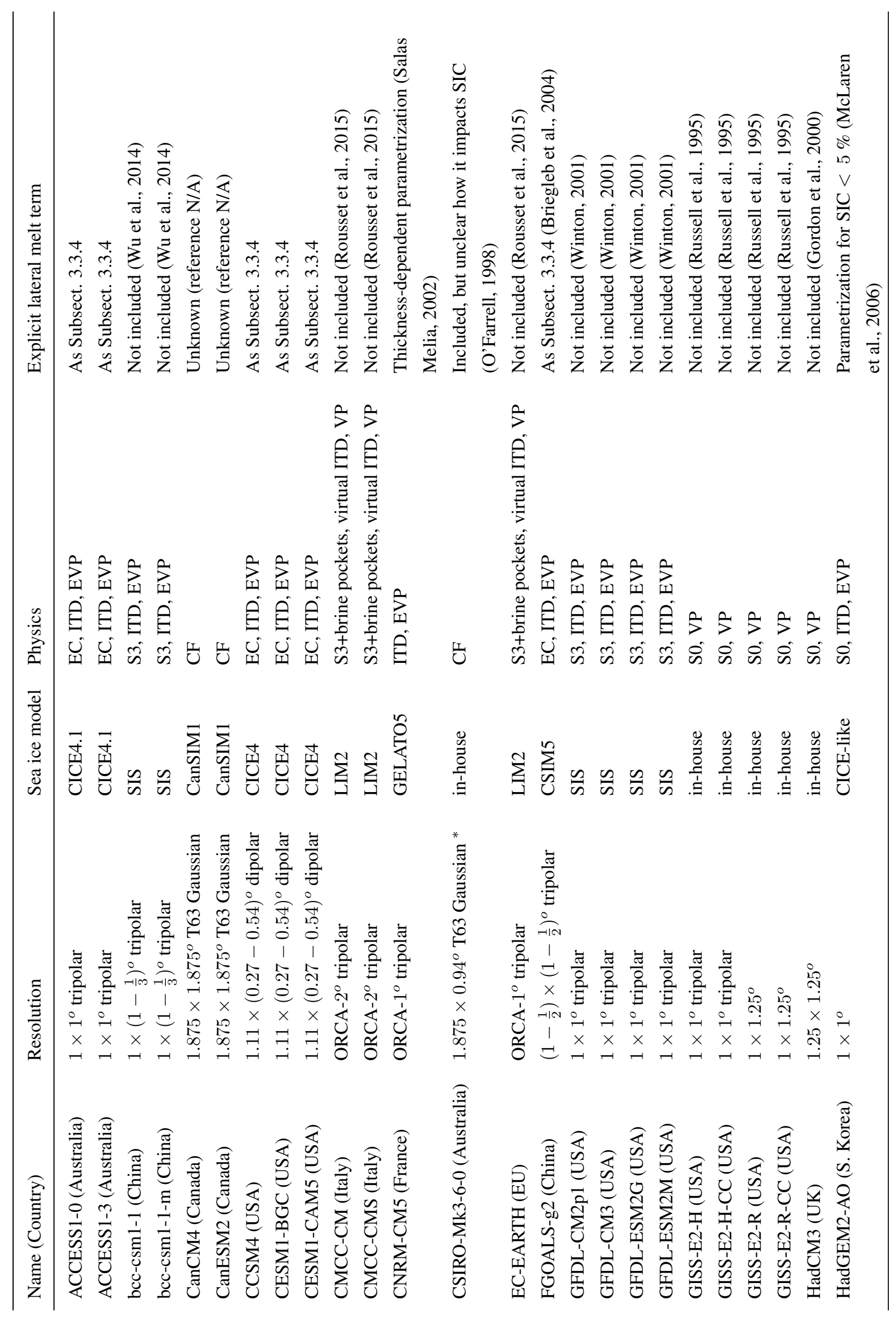




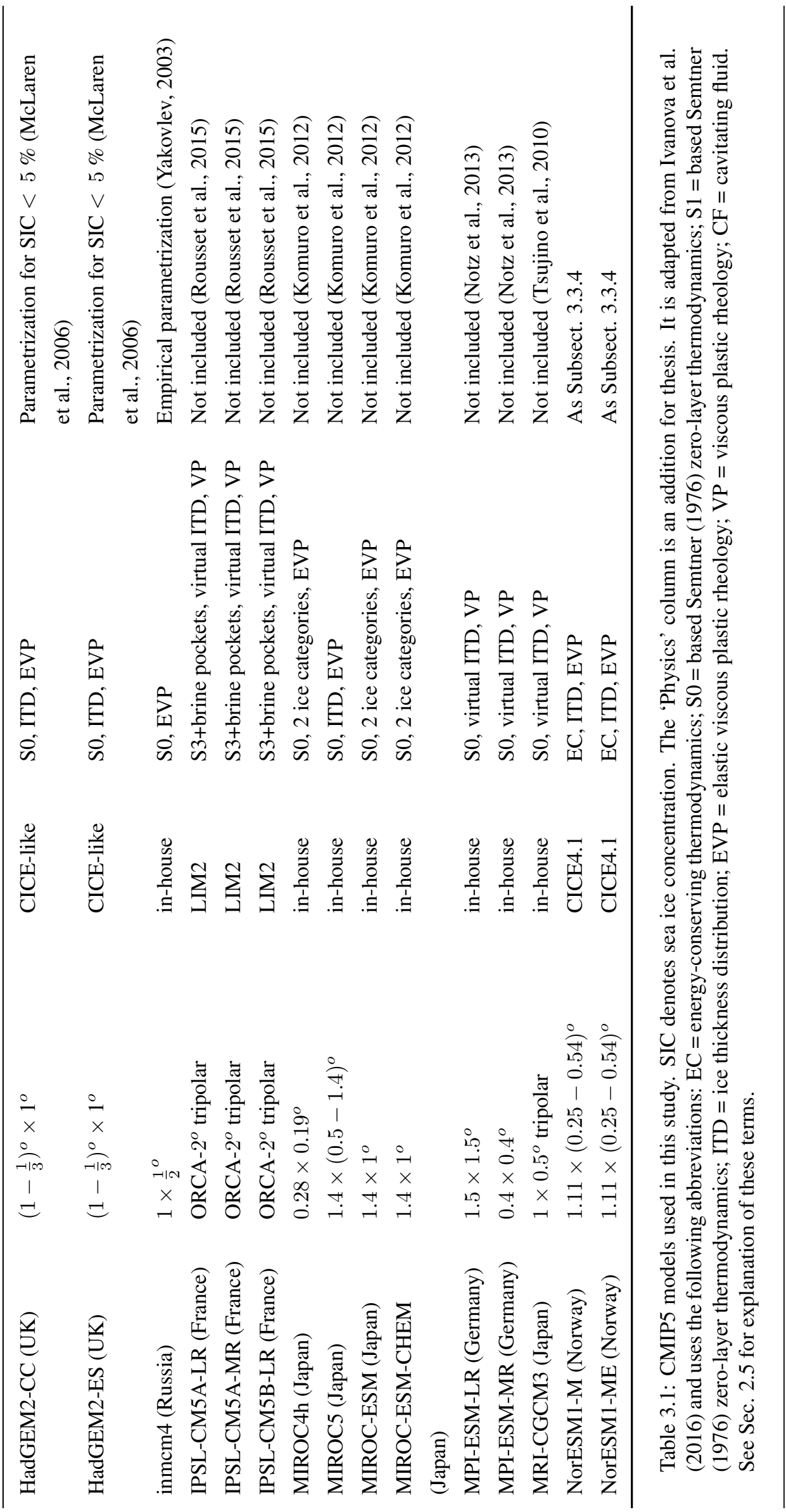


To account for some of this product uncertainty, we use three observational data sets: the Bootstrap algorithm (Comiso, 1986), the NASA Team algorithm (Cavalieri et al., 1984) and the ASI algorithm (Kaleschke et al., 2001; Spreen et al., 2008). We do not consider datasets that merge different observation methodologies. Bootstrap uses cluster analysis of brightness temperatures from two channels $(19 \mathrm{GHz}$ and $37 \mathrm{GHz}$ vertical polarization in the Antarctic), applies an ocean mask and is available from 1979 at a resolution of $25 \mathrm{~km}$. NASA Team uses ratios of brightness temperatures (which tends to cancel out physical temperature effects) from three channels (19 GHz in the vertical and horizontal, $37 \mathrm{GHz}$ in the vertical), removes weather contamination based on certain spectral gradient ratios and is available from 1979 at a resolution of $25 \mathrm{~km}$. The ASI algorithm uses the difference in brightness temperatures between horizontal and vertical polarization at $85 \mathrm{GHz}$, uses lower frequency channels at lower resolution to filter atmospheric effects (which are more apparent at $85 \mathrm{GHz}$ than lower frequencies), and is available from 1992 at a resolution of $12 \mathrm{~km}$. We choose to conduct our analysis over 1992-2014. Bootstrap and NASA Team data are available as monthly output; ASI-SSMI data is only available as daily output so the concentration fields are averaged for each month.

Differences between the three selected data sets are large: in the Antarctic, the NASA Team algorithm shows the marginal ice zone (defined as the extent of sea ice with concentration between $15 \%$ and $80 \%$ ) to extend over 2 million $\mathrm{km}^{2}$ more than the Bootstrap algorithm in the winter months (Stroeve et al., 2016). NASA Team is more sensitive to clouds and wind over open water than the Bootstrap mode (Andersen et al., 2006), while the high-frequency ASI algorithm is also sensitive to such atmospheric effects (Spreen et al., 2008). Bootstrap is more sensitive to physical temperature changes than NASA Team, and may underestimate concentrations at low temperatures, such as near the Antarctic coast (Comiso et al., 1997). For low concentrations, atmospheric effects, which generally lead to falsely increased sea ice, become increasingly important (Andersen et al., 2006). The weather filters/ocean masks used to correct these differ between the different algorithms.

Besides structural uncertainty in observational algorithms, systematic biases common to all three products are possible. Lack of validation data (Ivanova et al., 2014) mean it is difficult to quantify this, but accuracy is understood to be lower in the presence of 
melt ponds or other surface melt effects (Ivanova et al., 2014), which may act to lower retrieved concentrations; large fractions of thin ice (Ivanova et al., 2015); and stormy conditions near low concentrations (Andersen et al., 2006). Transitions between ice type can cause differences in emissivity (Grenfell and Comiso, 1986), but because models do not simulate ice types such as grease ice, this issue should not impact model-observation comparisons.

In this study, for some of the analysis we consider the three observational data sets individually. In order to compare the sea ice concentration distribution from the set of models against observations, we create an ensemble of the ASI-SSMI, Bootstrap and NASA Team observational products. Combining the observational products in this way does have limitations, as different algorithms are likely to perform better for certain sea ice conditions and seasons. However, it is not clear from the literature where exactly the strengths of the various algorithms lie, and evaluation of the different algorithms is beyond the scope of this manuscript. The difficulty in ranking various observational algorithms is noted by Ivanova et al. (2014), due to a lack of validation data. They recommend constructing an ensemble of different observational products.

\subsubsection{Metrics}

Following convention, sea ice extent is defined as the area of all grid cells with more than $15 \%$ sea ice concentration. Sea ice area is the fractional sea ice concentration multiplied by grid cell area, summed over all grid cells with more than $15 \%$ sea ice concentration

To account for misplacement of sea ice, we use the integrated ice-edge error (IIEE) from Goessling et al. (2016). The IIEE describes the area of grid cells where observations and a model disagree on the presence of sea ice with concentration greater than $15 \%$. It can be decomposed into the total sea ice extent difference between model and observations (absolute extent error, AEE) and the difference in sea ice extent due to misplacement of sea ice (misplacement extent error, MEE). See Goessling et al. (2016) for further details.

Here, we also define an integrated ice area error (IIAE) that describes the area of sea ice on which models and observations disagree. The ice area on which models and observations disagree is likely to be more physically relevant than the area of grid cells on 
which models and observations disagree. The IIAE is the sum of sea ice area overestimated and underestimated,

$$
\mathrm{IIAE}=O+U
$$

with

$$
O=\int_{A} \max \left(c_{m}-c_{o}, 0\right) d A
$$

and

$$
U=\int_{A} \max \left(c_{o}-c_{m}, 0\right) d A,
$$

where $A$ is the area of interest, $c_{m}$ is the simulated sea ice concentration and $c_{o}$ is the observed sea ice concentration.

The integrated ice errors are useful as they quantify error in integrated sea ice concentration values as well as quantifying error caused by sea ice appearing in different grid cells than the observations. This is in contrast to difference in sea ice area, which accounts only for error in integrated sea ice concentration values, and difference in sea ice extent, which accounts only for error in the area of grid cells that have ice. The integrated ice errors penalize under-estimation and over-estimation of sea ice equally.

In this study we also consider sea ice concentration distributions, as in Notz (2014) and Ivanova et al. (2016). The sea ice concentration distribution for each model or observational product is calculated by binning grid cells according to their concentration at a $10 \%$-spacing. The distribution is then normalized by the area of grid cells. We follow the same calculation steps as Notz (2014). This metric allows us to examine observed and modelled behaviour in different sea ice concentration regimes. It does not penalise models whose spatial distribution of sea ice disagrees with observations, but it does allow us to quantify disagreement with observations on sea ice concentration values while accounting for the observational range.

To look for behaviours which are consistent across all CMIP5 models, we compare the population of all models for the years 1992-2014 against the population of all observations for the same period. Including all models means that the range is large when models show opposite tendencies; using a multi-model mean would average out this information. Including all months in each season for all years during analysis captures sub-seasonal and inter-annual variability. 
To quantify the agreement between two populations, we use the two-sample KolmogorovSmirnov test. This compares the empirical distribution functions of each sample, and takes into account both the location and shape of the distributions. In contrast, a Student's t-test would only examine whether the means of the distributions agree. The p-value obtained from the Kolmogorov-Smirnov test represents the confidence that the two populations come from the same distribution.

We found that sea ice concentration distributions show some sensitivity to grid interpolation and therefore calculate sea ice concentration distributions, as well as sea ice area, on the native model and observation grids. The integrated ice errors and differences in sea ice concentration fields between models and observations must be calculated on the same grid. In these cases, we follow Turner et al. (2013) and interpolate model output and observational data on to a common grid using the bilinear remapping function from Climate Data Operators (CDO 2015). For the CMIP5 integrated ice errors and sea ice concentration differences, we choose a $1^{o} \times 1^{o}$ regular grid, which is a resolution equal to or higher than 20 of the 40 models and lower than all observations. We consider it to be an acceptable midpoint given the large range of model resolutions.

\subsubsection{Coupled ocean-sea ice model}

To understand the impact of model parametrizations for sea ice thermodynamics, we carry out perturbed parameter simulations using a coupled ocean-sea ice model. This consists of the ocean model NEMO coupled to the sea ice model CICE5.1 run on a $1^{\circ}$ tripolar grid, using a configuration based on Rae et al. (2015). The model is forced with $10 \mathrm{~m}$ specific humidity, wind speed and air temperature; surface downwelling shortwave and longwave radiation; precipitation; and sea level pressure fields from the atmospheric reanalysis JRA-55 (Japan Meteorological Agency, 2013). CICE is a state-of-the-art sea ice model and is used as the sea ice component for several of the CMIP5 models (Table 3.1). Below we briefly explain the model's sea ice thermodynamics; further details may be found in Hunke et al. (2015).

CICE describes the evolution of the ice thickness distribution in five discrete categories. A volume of new sea ice growth is calculated from the ocean freezing/melting 
potential $F_{\text {frz/mlt }}$, with new ice added as area in the smallest thickness category until the open water fraction is closed, after which it grows existing ice thickness. For sea ice melt, the net downward heat flux from the ice into the ocean, $F_{\text {bot }}$ is

$$
F_{\mathrm{bot}}=-\rho_{w} c_{w} c_{h} u_{*}\left(T_{w}-T_{f}\right)
$$

where $\rho_{w}$ and $c_{w}$ are the density and heat capacity of sea water, $c_{h}=0.006$ is the heat transfer coefficient, $u_{*}=\sqrt{\left|\overrightarrow{\tau_{w}}\right| \rho_{w}}$ is the friction velocity, $T_{w}$ is the sea surface temperature and $T_{f}$ is the ocean freezing temperature, following Maykut and McPhee (1995). The balance of this flux with a conductive flux through the ice determines basal melt.

A fraction of ice is also melted laterally following Steele (1992). If floes have a mean caliper diameter $L$, their perimeter is $p=\pi L$ and their horizontal surface area is $s=\alpha L^{2}$ (where $\alpha \approx 0.66$ accounts for the non-circularity of floes and was determined empirically by Rothrock and Thorndike 1984). It is assumed that melting occurs uniformly at a rate $w_{\text {lat }}$ around the perimeter of each floe, i.e.

$$
\frac{\mathrm{d} s}{\mathrm{~d} t}=w_{\text {lat }} p
$$

Therefore the change in diameter is

$$
\frac{\mathrm{d} L}{\mathrm{~d} t}=\frac{\pi}{2 \alpha} w_{\mathrm{lat}}
$$

For a region containing $n$ floes with only a single diameter $L$, with a total horizontal area $s_{\text {tot }}$, the total concentration $A$ is

$$
A=\frac{n}{s_{\text {tot }}} s(L)=\frac{n}{s_{\text {tot }}} \alpha L^{2}
$$

Hence, with $s_{\text {tot }}$ and $n$ constant in time and letting the subscript ${ }_{o}$ denote the initial state,

$$
A=A_{o}\left(\frac{L}{L_{o}}\right)^{2}
$$

Differentiating this and inserting $d L / d t$ then gives the change in concentration,

$$
\frac{\mathrm{d} A}{\mathrm{~d} t}=\frac{A \pi}{L \alpha} w_{\text {lat }} .
$$


CICE uses a uniform lateral melt rate of

$$
w_{\text {lat }}=m_{1}\left(T_{w}-T_{f}\right)^{m_{2}},
$$

which was based on Josberger and Martin (1981), who found a complex boundary layer adjacent to vertical ice walls melting in saltwater in the laboratory, with convective motions following different flow regimes. The region adjacent to the turbulent flow regime showed the largest lateral melt rate, which could be fitted to the above relation. The coefficients $m_{1}$ and $m_{2}$ are the best fit to data quoted by Maykut and Perovich (1987), measured in a single static lead in the Canadian Arctic archipelago over a three week period. In order to apply Eq. 3.6, CICE assumes a single floe diameter of $L=300 \mathrm{~m}$ throughout the ice pack. This is one of the more sophisticated schemes for lateral melt in the CMIP5 models; often it is not included at all (Table 3.1).

The experiments described below, which are performed with the coupled NEMOCICE model, begin in 1979 and end in 2014. The years before 1992 are neglected to allow for model spin-up. Time series of annual maximum sea ice extent show that this takes around ten years to stabilize. Model output from the NEMO-CICE experiments is analysed on its native grid ( $1^{o}$ tripolar). Comparisons between NEMO-CICE simulations and observations (integrated ice errors and sea ice concentration differences) are computed by interpolating observations on to the same $1^{\circ}$ tripolar grid using CDO (2015).

\subsection{Results}

Fig. 3.1 shows sea ice area at the annual maximum and minimum from models and observations. Examining observations and models shown individually (Fig. 3.1a and 3.1c), we find that the interquartile range arising from inter-annual fluctuations over 1992-2014 is generally smaller than inter-model differences. 

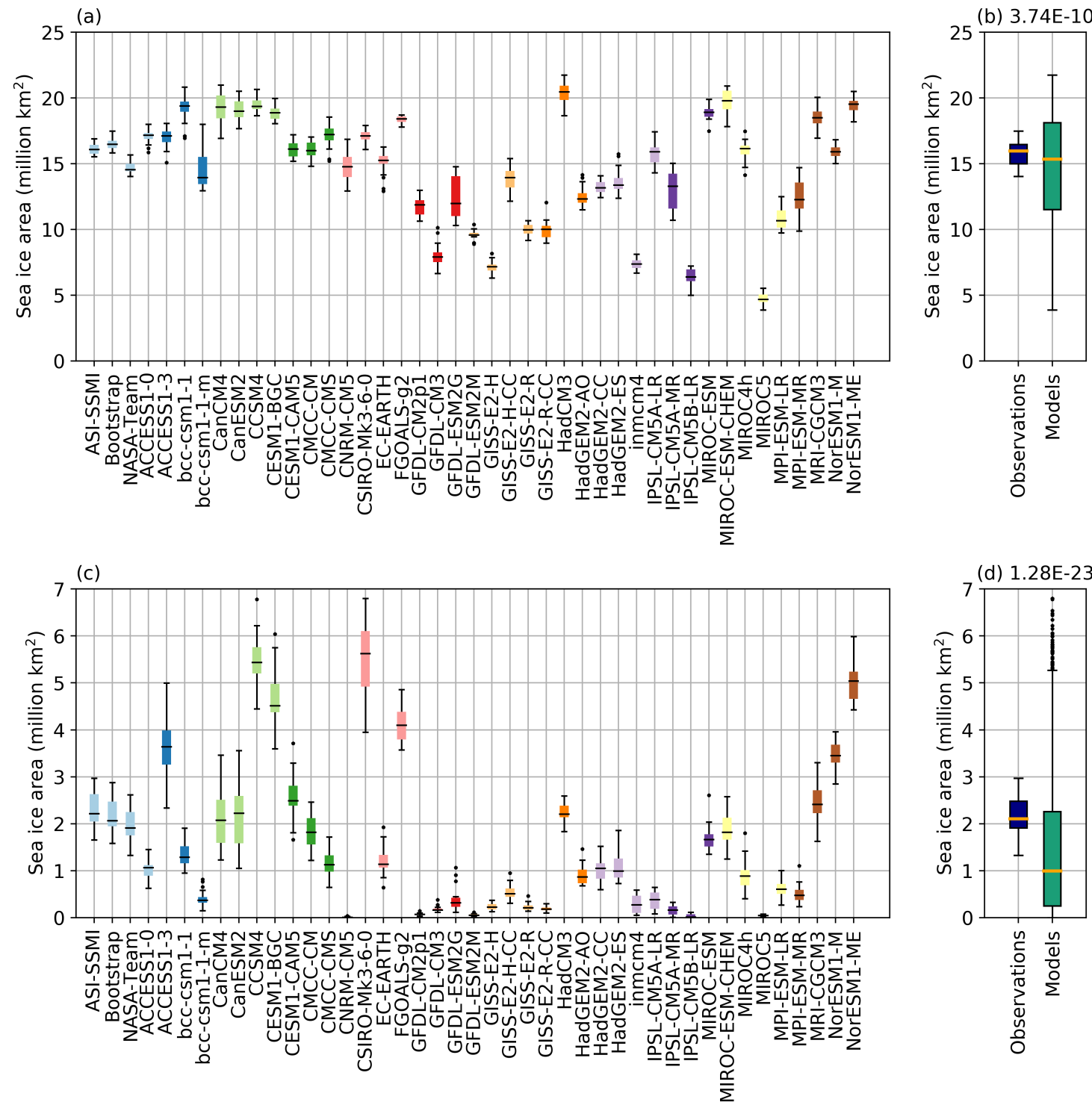

Figure 3.1: Sea ice area for the months where the maximum (a-b) and minimum (c-d) of the seasonal cycle occur. Populations include data from all years from 1992 to 2014 with boxplots for $(\mathrm{a}, \mathrm{c})$ the three observational products (ASI-SSMI, Bootstrap and NASA Team) and all CMIP5 models listed in Table 3.1 individually; and $(\mathrm{b}, \mathrm{d})$ for the ensemble of observational products and the CMIP5 model ensemble. Boxes extend from the lower to upper quartile values of the data with a line at the median. Whiskers show 1.5 of the interquartile range; beyond this data are considered outliers and plotted as individual points. The text labels in $(\mathrm{b}, \mathrm{d})$ is the $\mathrm{p}$-value calculated from a Kolmogorov-Smirnov test, which represents the confidence that the two populations come from the same distribution. 
Fig. 3.1b and 3.1d group the models and observations into two populations for comparison. At the annual maximum (Fig 3.1b), the interquartile range from the ensemble of observations for 1992-2014 is contained within the ensemble of models from the same period, with the medians of the two populations in good agreement. There is no clear model tendency compared to observations for the sea ice area maximum. At the minimum (Fig. 3.1d), the interquartile ranges from models and observations show less overlap than the maximum, with the median from the model ensemble significantly lower than the median from the observational ensemble, suggesting a broadly consistent underestimation of sea ice area at the annual minimum by the CMIP5 models. This tendency was also noted by Turner et al. (2013) for sea ice extent. There are outliers, which show an overestimation of sea ice area, notably CSIRO-Mk-3-6-0 and the CESM models. The Kolmogorov-Smirnov test quantitatively shows that both the maximum and minimum sea ice area model-observation comparisons are significantly different, but the difference between models and observations is larger at the summer minimum than at the winter maximum (Figs. 3.1b and 3.1d).

The poorer performance of models at the summer minimum is supported by the integrated ice area error (Fig. 3.2a). In the summer, the model median sea ice area minimum is around 1 million $\mathrm{km}^{2}$ while the model median integrated ice area error at this time is around 2 million $\mathrm{km}^{2}$; at the winter maximum, the area is around 15 million $\mathrm{km}^{2}$ and the error is around 6.5 million $\mathrm{km}^{2}$. Results are similar using the integrated ice extent error (Fig. 3.2b), although the use of extent rather than area reduces the variation between observational references. At the winter maximum, across the population of CMIP5 models and different years, we find that the absolute extent error and the misplacement extent error contribute approximately equally to the total integrated ice extent error (Fig. 3.2c-d). At the summer minimum, the integrated ice extent errors for the CMIP5 models have a slightly larger contribution from absolute extent errors than from misplacement area errors (Fig. 3.2c-d).

The large inter-model variability in extent and area at the summer minimum can be seen in Fig. 3.3, where the sea ice concentration fields show diverse behaviour. Variability between observational products is smaller than inter-model differences, but observational differences are visible, particularly at low concentrations. An objective way to quantify model-observation disagreement is to use the integrated ice area error, which describes 

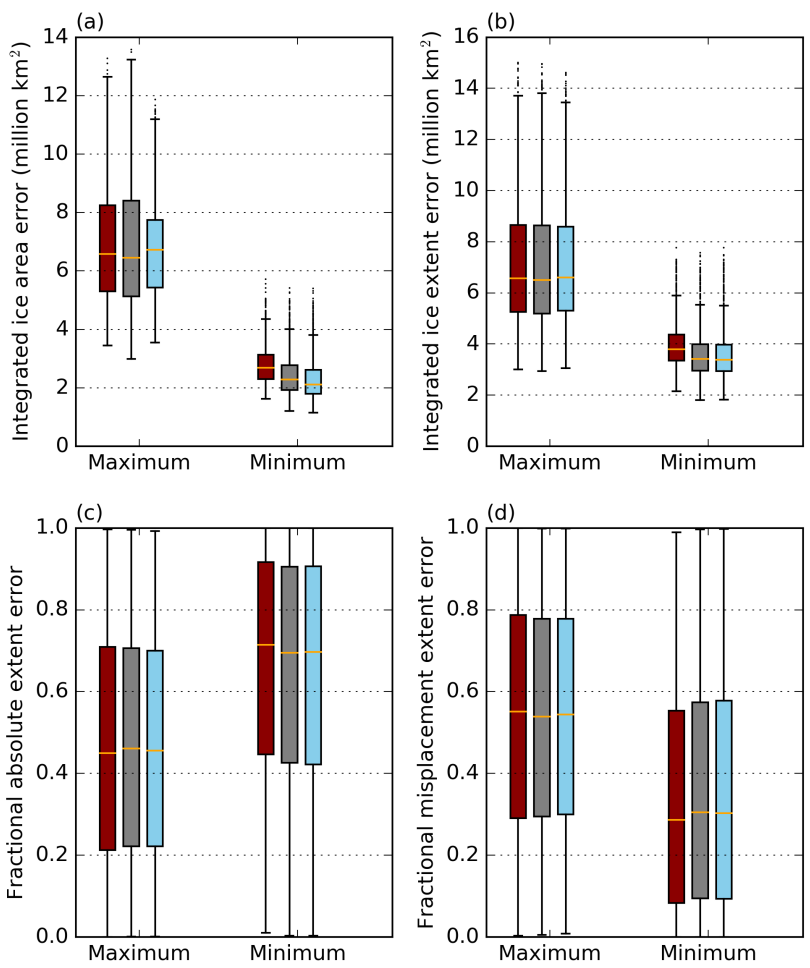

Relative to ASI-SSMI

Relative to Bootstrap

Relative to NASA-Team

Figure 3.2: Various ice errors for the population of CMIP5 models for all years from 1992 to 2014. Errors are shown relative to (red) the ASI-SSMI satellite observations, (grey) the Bootstrap satellite observations and (light blue) the NASA-Team observations for the months where the maximum and minimum of the seasonal cycle occur of sea ice area (a) or of sea ice extent (b-d) occur. The errors shown are the integrated ice area error (a), the integrated ice extent error (b), the absolute extent error divided by the integrated extent error (c) and the misplacement extent error divided by the integrated extent error (d). Boxplots are as in Fig. 3.1.

the area of sea ice on which models and observations disagree. Due to observational variability, we calculate this relative to each observational product individually. The variation in observations means that we cannot rank the models in an overall order, but we can construct two groups of well-performing models and of poorly-performing models whose members do not change when using different observational products. These are marked on Fig. 3.3.

We now consider sea ice concentration distributions from observations and models, which provide a more detailed assessment than hemisphere-integrated measures. A normalized sea ice concentration distribution may help isolate the role of the sea ice component, as models with a constant temperature bias in the atmosphere or ocean, resulting in 


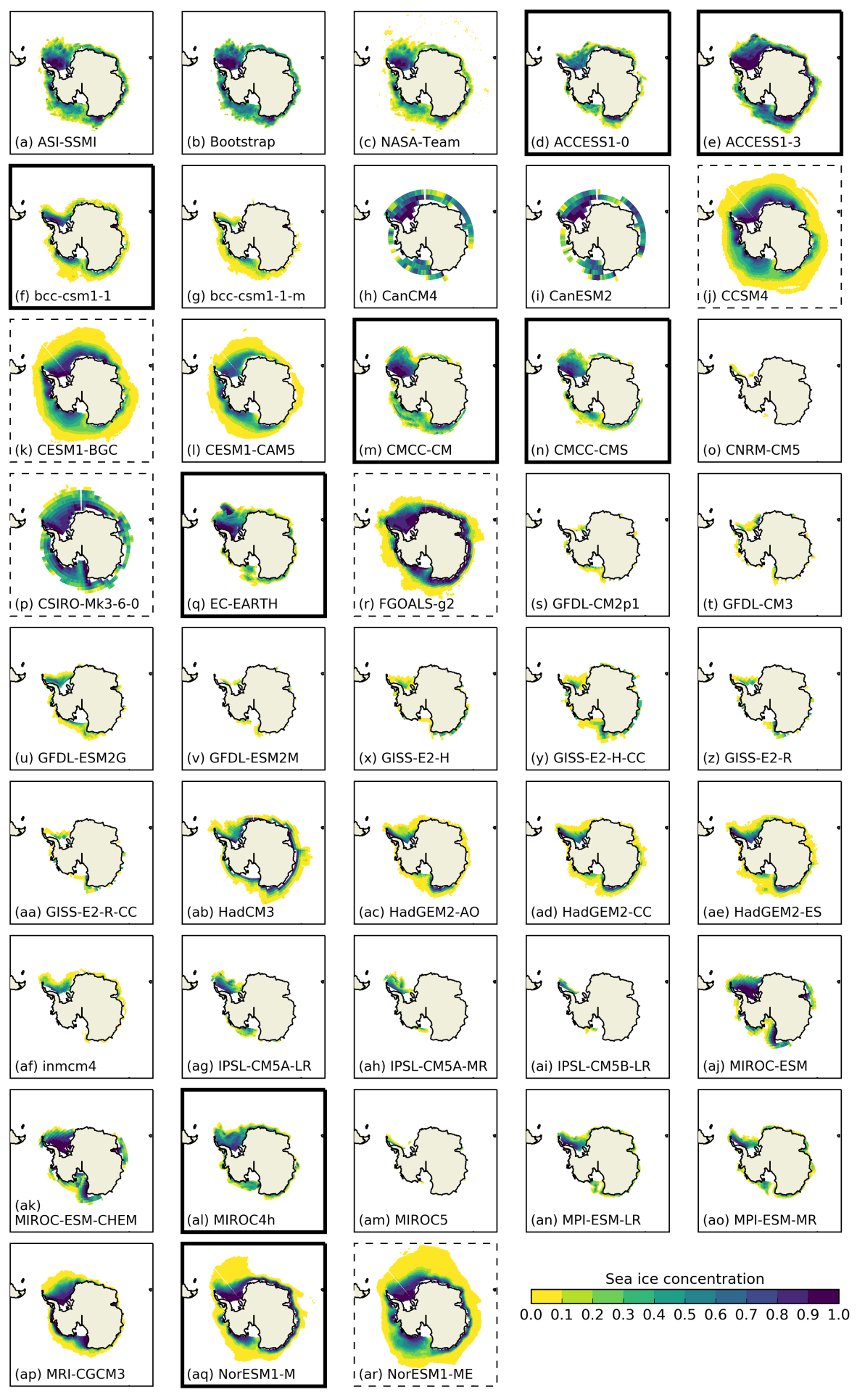

Figure 3.3: Sea ice concentrations (above $0.1 \%$ ) for the three sets of observations (a-c) and the CMIP5 models (d-ar) for the month of each model or observation's sea ice area minimum, averaged over 19922014. Models marked with a bold (dashed) bounding box have high-ranked (low-ranked) integrated ice area errors regardless of observational product used. Integrated ice area errors consider sea ice concentrations > $15 \%$ for the sea ice field shown. 
a biased sea ice area or extent, may still simulate the relative fraction of different concentration regimes successfully.

As shown by Ivanova et al. (2016), the CMIP5 multi-model mean and the NASA Team observations have a high fraction of ice below $10 \%$ sea ice concentration in the summer. We find that the fraction of 0.001-10\%-concentration ice varies in the models from 0.005 to 1.0 (when models are essentially ice-free) in the summer (Fig. 3.3). It consists of up to around a third of the ice in other seasons for some models. Including these very low concentrations heavily skews the normalized sea ice concentration distribution towards low concentrations and it obscures behaviour at higher concentrations. Our aim is to look for consistent model behaviour, so to avoid the large variance between different models and between different observations at very low concentrations, we only consider sea ice concentrations above $10 \%$. We present all months grouped by meteorological season (December-February (DJF), March-May (MAM), June-July (JJA) and September-November (SON)). This choice separates the melt season (Sep-Feb) from the freezing season (Mar-Aug), while limiting the number of months included in each season.

We first describe satellite observations using the normalized sea ice concentration distribution (Fig. 3.4). Here, individual boxplots contain both inter-annual and sub-seasonal variability, while the differences between boxplots reflects uncertainty arising from different processing of satellite data. Differences between observational products are larger for compact ice $(90 \%+)$ than other concentrations. In general, the ASI-SSMI observations show more similar characteristics to the Bootstrap observations than the NASA Team observations for most of the year, apart from DJF where the opposite is true. This results in a somewhat skewed distribution when considering an ensemble created from three data sets. We find that the NASA Team algorithm shows a looser ice cover, with a significantly lower proportion of cover in the $90 \%+$ concentration bin, than both the Bootstrap and ASI-SSMI observations. This result holds when considering an un-normalized sea ice concentration distribution as well (not shown). The fraction in the 70-90\% bins is larger to compensate. We also find that differences between data sets persist throughout the year. This is in contrast to the Arctic, where the frequency of compact sea ice cover shown in the Bootstrap and NASA Team datasets shows largest disagreement in the summer, due to issues with treatment of melt ponds (Notz, 2014). In the Antarctic, observational uncertainty in the frequency of compact sea ice is largest in winter. 

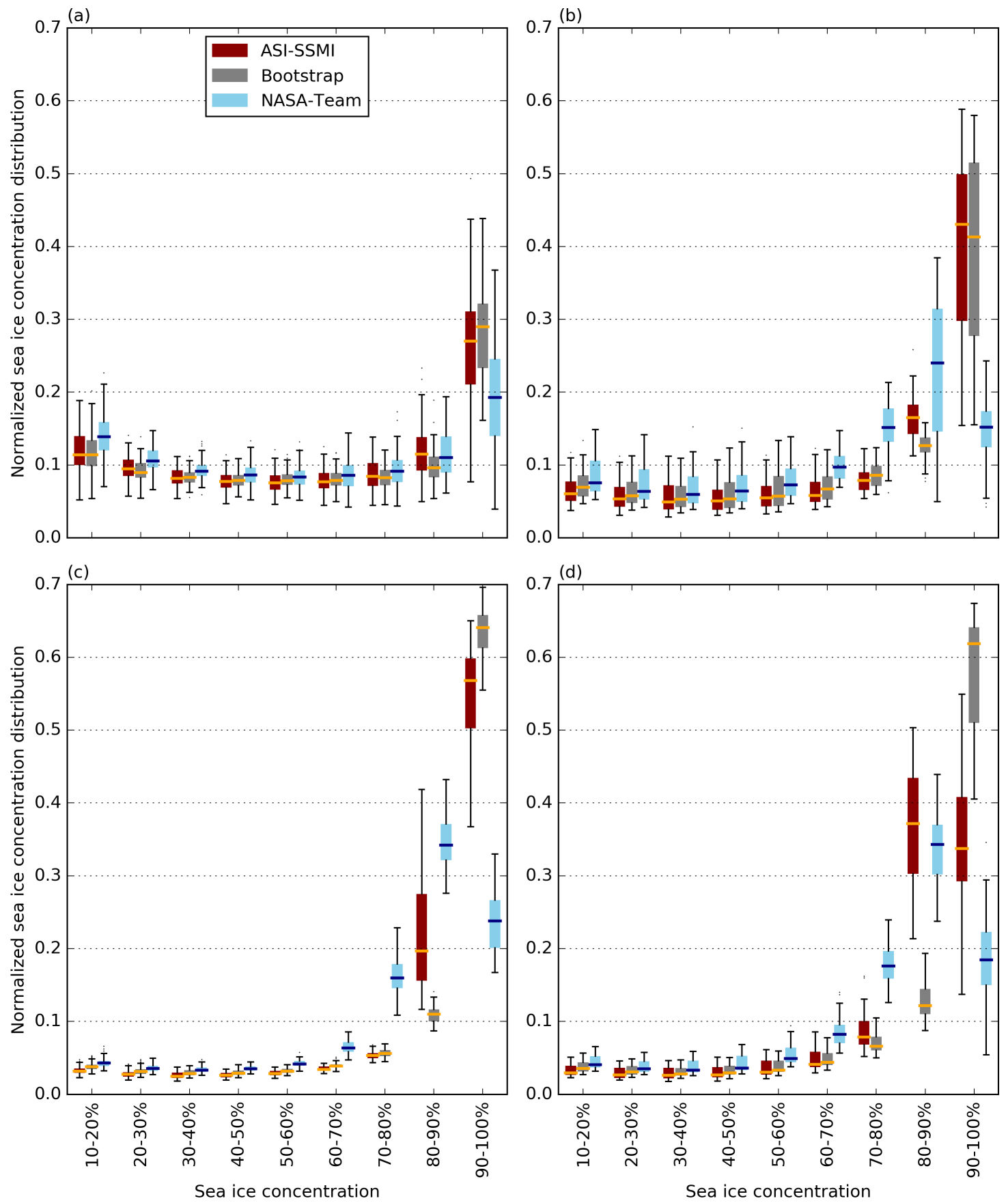

Figure 3.4: The normalized sea ice concentration distribution for all months in each year from 1992 to 2014 in (a) DJF, (b) MAM, (c) JJA, and (d) SON from the three sets of satellite observations. Boxplots as in Fig. 3.1 . 
Differences between the sea ice concentration distribution from models and observations, including inter-annual and sub-seasonal information, (Fig. 3.5) are less distinct than between observational products themselves. This reflects the large range in both models and observations due to systematic uncertainties. The overall decomposition from the CMIP5 models, with a large fraction of compact ice cover and smaller fractions of lower concentrations is somewhat in agreement with observations. Agreement appears poorest in DJF, where the lower to upper quartile range for $90 \%+$ sea ice concentration from models and observations overlap very little. Models strongly underestimate the fraction of sea ice area with concentration greater than $90 \%$, that is, their central ice pack is not compact enough. They tend to overestimate the fraction in the 80-90\% bin and at lower concentrations to compensate. In other seasons, there appears to be a slight tendency to overestimate the fraction of compact $(90 \%+)$ ice, with a reduction in the $70-90 \%$ bins to compensate. The two-sample Kolmogorov-Smirnov test can be used to quantify the degree of disagreement between models and observations. The confidence level that the ensemble of observations and ensemble of models were drawn from the same population has the smallest values for the 90-100\% and 10-20\% concentrations in DJF, the 70-90\% concentrations in MAM, the 10-30 \% concentrations in JJA and the 80-90\% and 10-20 $\%$ concentrations in SON. There is a tendency for models to overestimate the fraction of low-concentration (10-20\%) sea ice in all seasons. This overestimation of $<20 \%$ sea ice compared to observations is robust when considering sea ice concentration bins spaced at $5 \%$ intervals and beginning at $15 \%$, the cut-off used universally for sea ice extent (not shown). Unlike the other CMIP5 model tendencies, the overestimation of 10-20\% ice occurs in every month (Fig. 3.6), with the CMIP5 model median always outside the interquartile range of the observations.

As discussed above, this assessment takes into account observational uncertainty and inter-annual and sub-seasonal variability. That distinct tendencies arise from a population of 40 models, which contain diverse physics and different sea ice, ocean and atmosphere models, is striking. It suggests that there is some deficiency or missing physical process common to many models. 


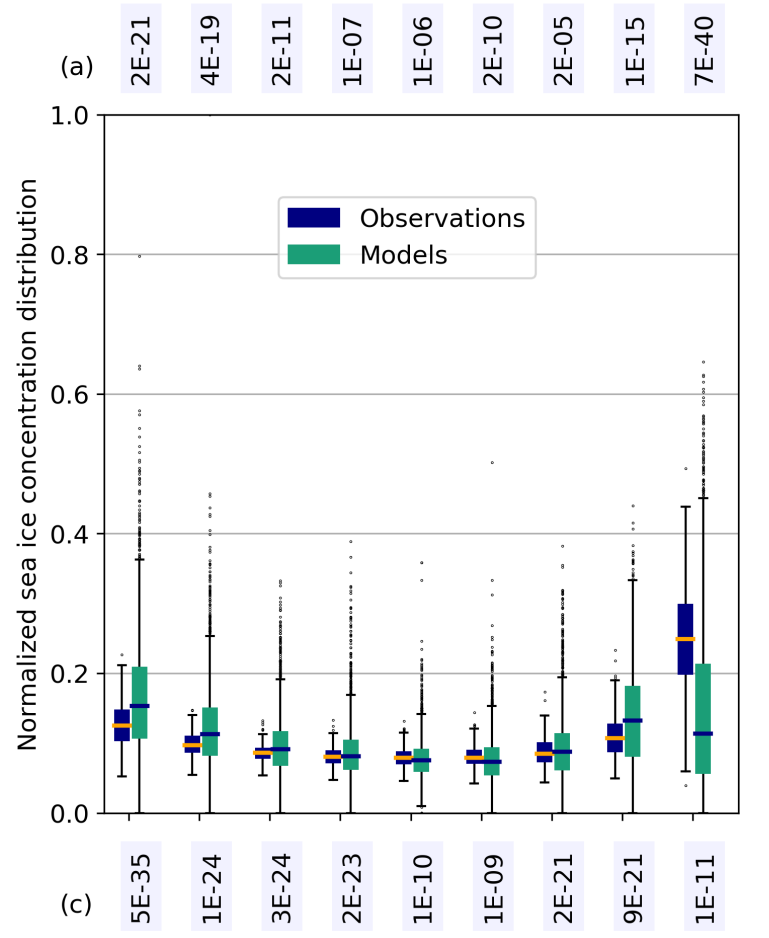

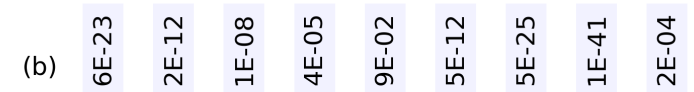
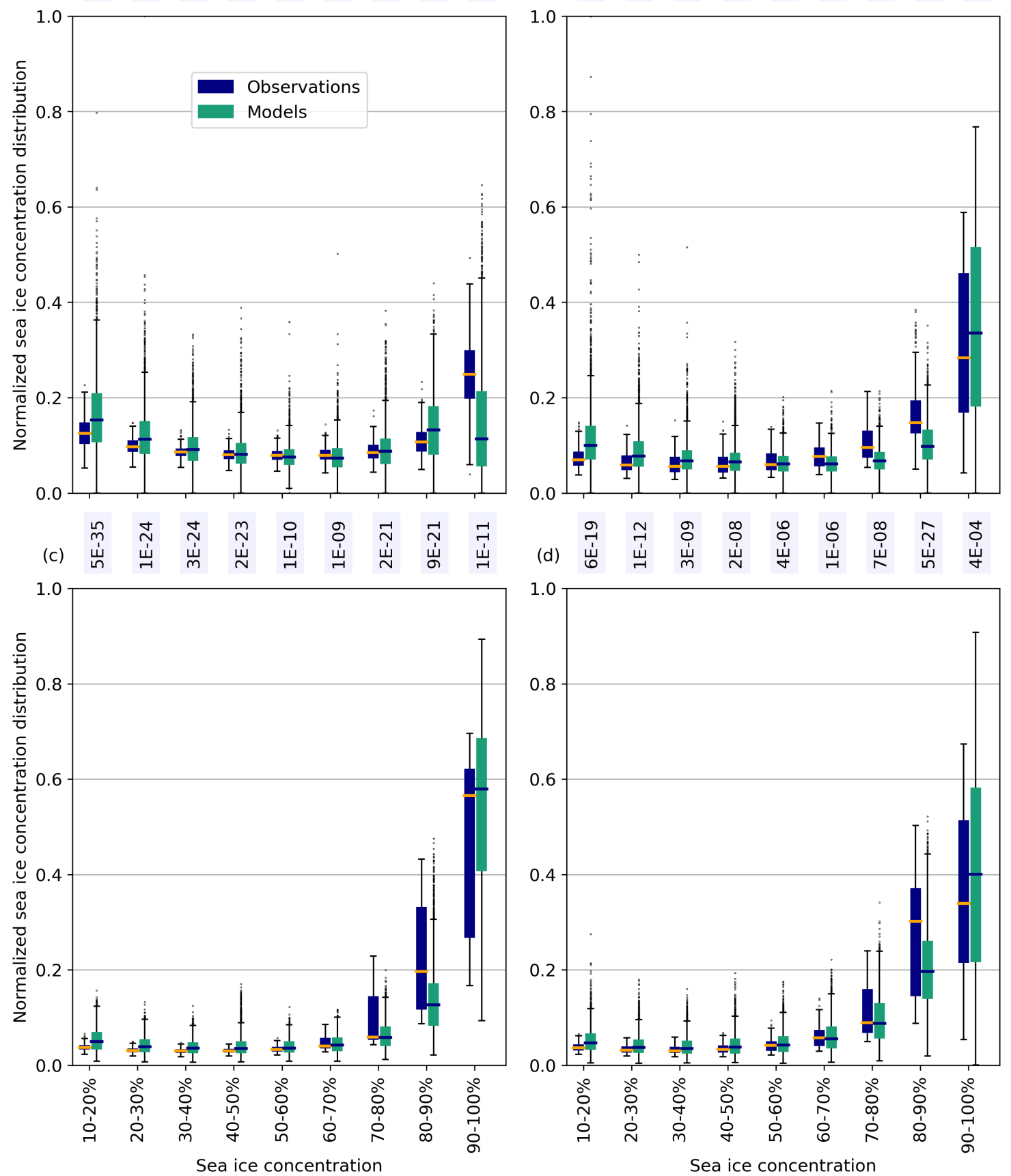

Figure 3.5: The normalized sea ice concentration distribution for all months in each year from 1992 to 2014 in (a) DJF, (b) MAM, (c) JJA, and (d) SON from the the three sets of satellite observations (blue) and the 40 CMIP5 models (green). Boxplots as in Fig. 3.1. Annotated text is the p-value calculated from a Kolmogorov-Smirnov test, which represents the confidence that the two populations come from the same distribution. 

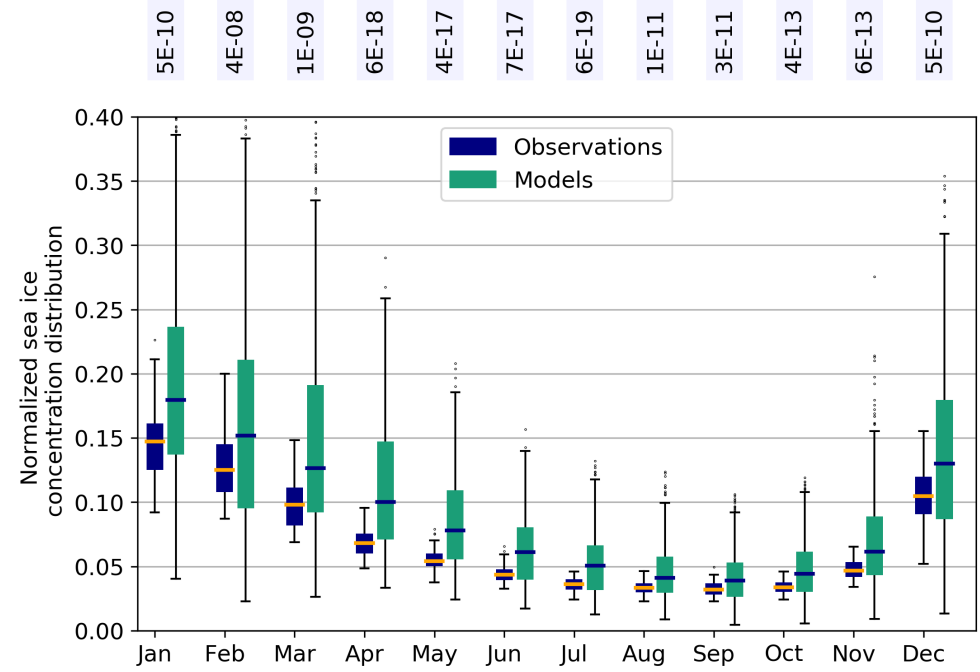

Figure 3.6: The 10-20\% bin from the normalized sea ice concentration distribution for each month, where boxes contain all years from 1992 to 2014 from (blue) the the three sets of satellite observations and (green) the 40 CMIP5 models. Boxplots as in Fig. 3.1. Annotated text is the p-value calculated from a KolmogorovSmirnov test, which represents the confidence that the two populations come from the same distribution.

A plausible explanation could be that models form sea ice that is too thin in the highest bin, which therefore melts more easily. Conversely, low-concentration sea ice may be too thick. However, we found no relation between these concentration biases and average sea ice thickness for the lowest and highest concentration bins (not shown). We therefore turn to lateral, rather than vertical, thermodynamics in the next section.

\subsection{Impact of floe size}

We hypothesize that the biases in low-concentration Antarctic sea ice are significantly influenced by lateral floe size. Lateral floe size impacts sea ice concentration through lateral melt only, if included at all in the CMIP5 models (see Table 3.1). Separating models with and without an explicit lateral melt term (Table 3.1), we find a significant difference between the two groups. Models with explicit lateral melt show a greatly reduced fraction of low-concentration ice from March to July compared to models without, in good agreement with the observations (Fig. 3.7). Lateral melt can occur all year at the ice edge, where low concentrations occur. 


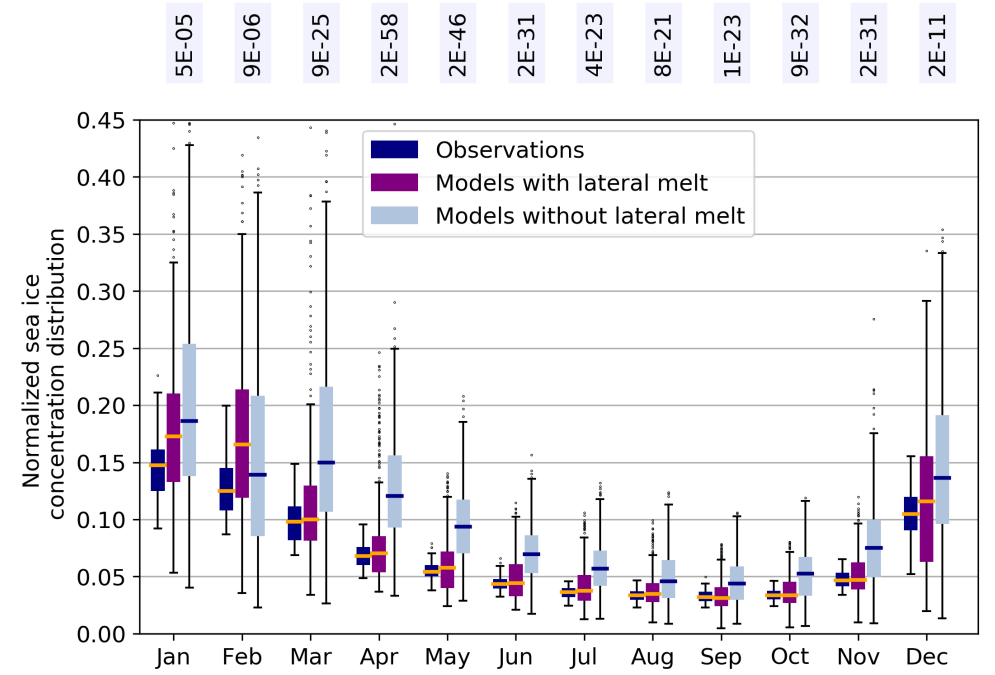

Figure 3.7: The 10-20\% bin from the normalized sea ice concentration distribution for each month, where boxes contain all years from 1992 to 2014 from (blue) the three sets of satellite observations, (purple) CMIP5 models that include an explicit lateral melt term and (grey) CMIP5 models that do not (from Table 3.1). Boxplots as in Fig. 3.1. Annotated text is the p-value calculated from a Kolmogorov-Smirnov test, which represents the confidence that the two model populations come from the same distribution.

Fig. 3.7 demonstrates that lateral melt significantly impacts the normalized sea ice concentration distribution during autumn. However, lateral melt as it is currently included in CMIP5 models still results in a tendency towards overestimation of low-concentration sea ice in other months, and some models with an explicit lateral melt term (including the ocean-sea ice model NEMO-CICE) still simulate too large a fraction of loose ice.

We therefore proceed by examining whether changes to the lateral melt scheme may also impact the simulation of sea ice. The current representation of lateral melt in CMIP5 models is heavily parametrized (Table 3.1), with the formulation described in Subsect. 3.3.4 being the most complex parametrization available in the CMIP5 models. Tsamados et al. (2015) showed that a more advanced concentration-dependent lateral melt parametrization significantly impacted the decomposition of sea ice melt processes, resulting in reduced sea ice concentrations around the ice edge in the Arctic. In the Antarctic, heat flux from solar heating of open water areas has been cited as the major cause of sea ice decay (Nihashi and Ohshima, 2001), with this melting potential available for both lateral and bottom melt. Recent studies have also suggested that floe size should also impact sea ice concentration through processes such as floe-floe collisions and lateral growth (Horvat and Tziperman, 2015; Zhang et al., 2015). 
As shown in Subsect. 3.3.4, in CICE the lateral melt flux is independent of floe size, while the change in concentration arising from lateral melt is inversely proportional to a constant floe diameter, $D=300 \mathrm{~m}$. In reality, sea ice floes can range in size across orders of magnitude. Several observational studies (e.g. Steer et al. 2008; Paget et al. 2001) find that the number distribution of floe sizes per unit area follows a power law with a negative exponent, suggesting that there can be a large number of small floes.

While concentration is not a proxy for floe size, in general we may expect that lowconcentration areas will be made up of smaller sea ice floes than high-concentration areas because they are usually nearer the ice edge. An area of smaller sea ice floes will experience more lateral melt than an area with a larger floe size (Eq. 3.6). We therefore suggest that CMIP5 models using the Steele (1992) lateral melt parametrization simulate too much low-concentration sea ice because this is made up of floes smaller than 300 $\mathrm{m}$ and so should be subject to more lateral melt. In areas around the ice edge, which are principally low-concentration, marginal ice zone processes not included in CMIP5 models, such as wave fracture and dynamic floe interactions, may further reduce concentrations. Conversely, in high-concentration areas, floes are likely to be larger than $300 \mathrm{~m}$ and therefore should be subject to less lateral melt than the Steele (1992) parametrization prescribes. This could explain the underestimation of high-concentration sea ice seen in Antarctic summer.

In order to test this hypothesis, we perform three experiments using the coupled ocean-sea ice model (NEMO-CICE) described in Subsect. 3.3.4. The experiments have identical set ups apart from a variation in $L$, the fixed floe diameter. We run experiments using (i) the standard value of $L=300 \mathrm{~m}$, (ii) a low value of $L=1 \mathrm{~m}$ and (iii) a high value of $L=10,000 \mathrm{~m}$. Our perturbed parameter values are constant and not realistic, but instead are chosen to investigate and highlight the impact of extreme changes.

Fig. 3.8 shows the fraction of $10-20 \%$ sea ice concentration from observations, the standard NEMO-CICE model and the model with reduced floe size. The standard model has very strong overestimation of low-concentration ice through December to March compared to observations. Impact of reduced floe size on the distribution is limited, with the exception of February where there is a very strong reduction in the fraction of 10-20\% concentration ice, bringing it into better agreement with observations. 


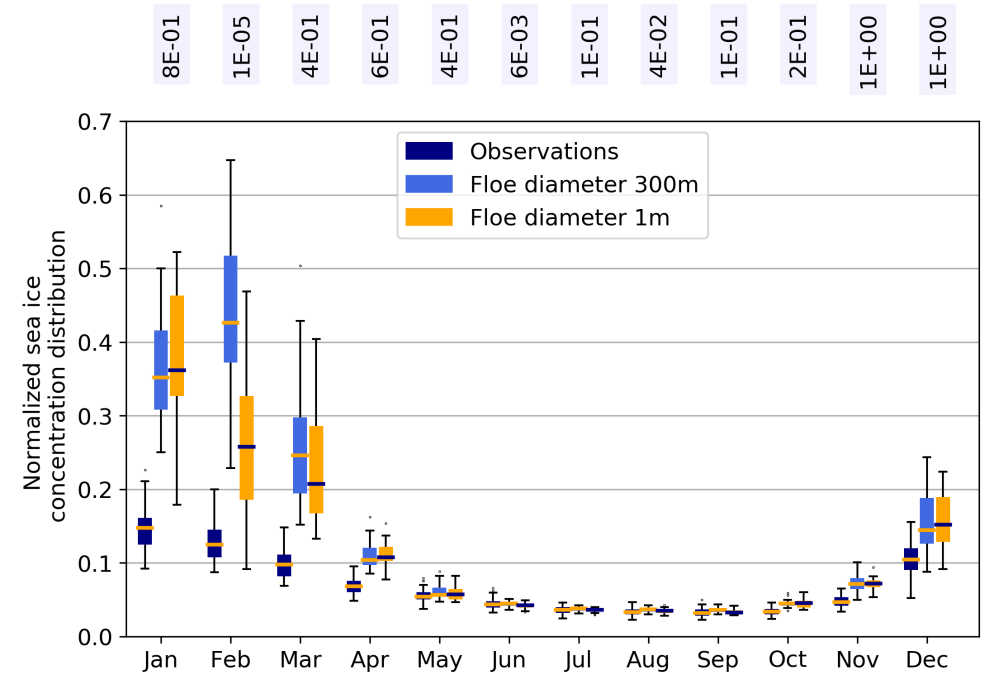

Figure 3.8: The 10-20\% bin from the normalized sea ice concentration distribution for each month, where boxes contain all years from 1992 to 2014 from a NEMO-CICE simulation with (blue) the the three sets of satellite observations, (light blue) a floe diameter of $300 \mathrm{~m}$ (the standard model) and (orange) a floe diameter of $1 \mathrm{~m}$. Boxplots as in Fig. 3.1. Annotated text is the p-value calculated from a Kolmogorov-Smirnov test, which represents the confidence that the two model populations come from the same distribution.

The enhanced lateral melt achieved by reducing floe size results in statistically significant reductions in sea ice concentration relative to the standard model in DJF (Fig. 3.9b). December, January and February stand out from the other months in having particularly high total lateral melt rates. As expected from Fig. 3.6, enhanced lateral melt reduces the high bias in concentration near the outer ice edge compared to Bootstrap observations in DJF (reduction in blue, Fig. 3.9d-e), but enhances the low bias compared to the Bootstrap observations elsewhere (increase in red, Fig. 3.9d-e). We use the integrated ice extent error described above to quantify agreement with the Bootstrap observations. The same qualitative picture is obtained from all three observational products. We find that the difference in overall agreement with observations between the standard model and the small floe simulation is negligible. The absolute extent error significantly increases in the small floe simulation, because overall this simulation melts too much ice compared to observations. The misplacement extent error, however, is significantly reduced in the small floe simulation. This is partly because there is less ice to be misplaced, but also because increased lateral melt improves the distribution of sea ice around the ice edge, by melting areas where there is too much ice compared to observations (Fig. 3.9d-e). 

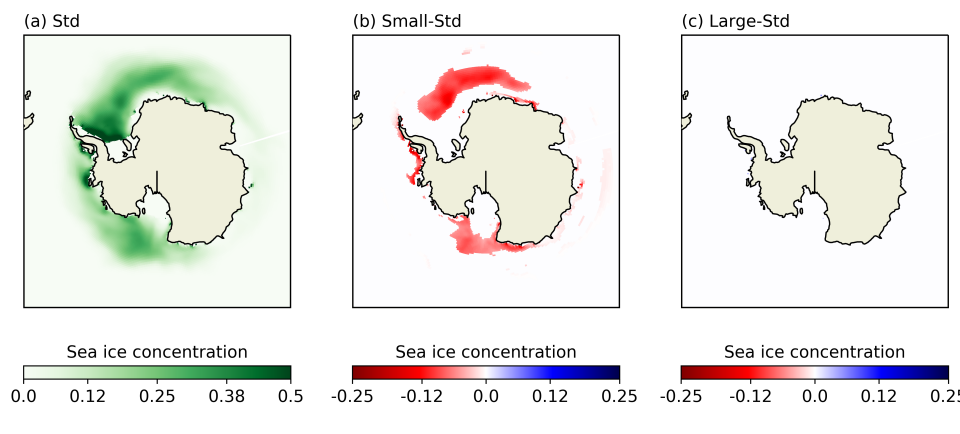

Sea ice concentration

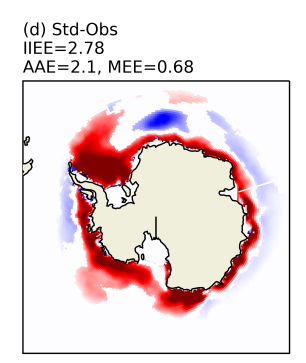

(e) Small-Obs

$\mathrm{MIEE}=3.07$
$\mathrm{AAE}=2.74, \mathrm{MEE}=0.33$
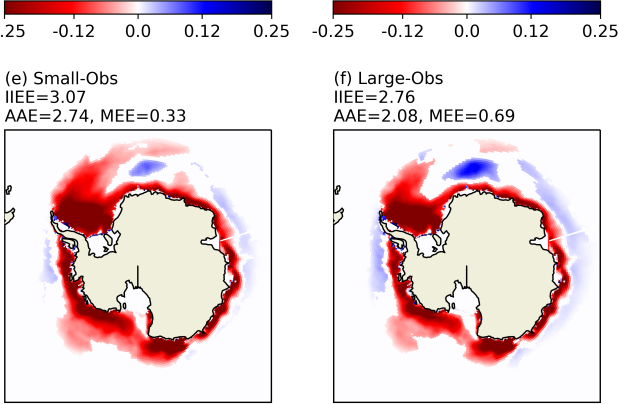

(f) Large-Obs

$\mathrm{IEE}=2.76$
$\mathrm{AAE}=2.08, \mathrm{MEE}=0.69$

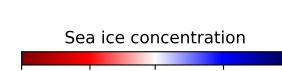

Sea ice concentration
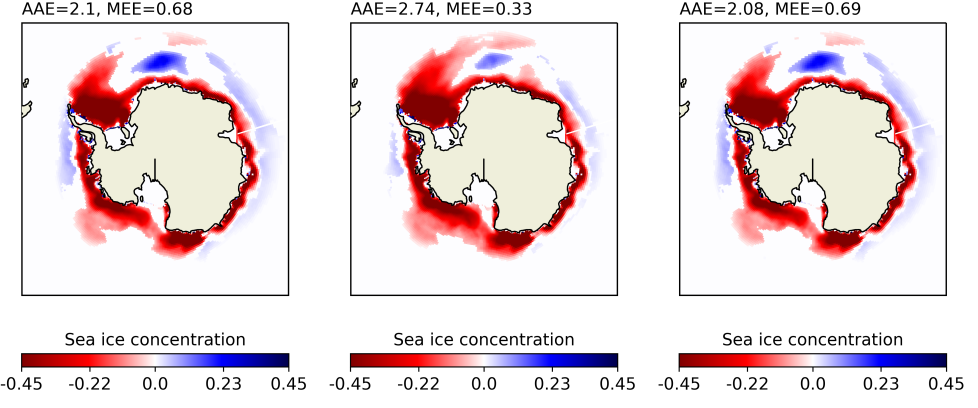

Figure 3.9: Sea ice concentration averaged over DJF 1992-2014 for (a) the standard model simulation with a floe diameter of $300 \mathrm{~m}$ (Std); (b) a model simulation with a floe diameter of $1 \mathrm{~m}$ (Small) minus (a); (c) a model simulation with a floe diameter of $10,000 \mathrm{~m}$ (Large) minus (a). (d-f) show simulation minus observed Bootstrap sea ice concentration (Obs), where the latter has been interpolated on to the model grid for (d) the standard model simulation, (e) the small floes simulation and (d) the large floes simulation. In (b-f), differences are shown only if they are statistically different according to a Student's t-test over 1992-2014 $(p<5 \%)$. Labels on (d-f) show the integrated ice extent error, absolute extent error and misplacement extent error in million $\mathrm{km}^{2}$.

Besides lateral melt, a number of other physical processes, including dynamical ones, may also contribute to an overestimation of low-concentration ice. Lecomte et al. (2016) find systematic wind-driven biases in sea ice drift speed and direction at the exterior of the Antarctic ice pack. Errors in surface winds could contribute to poor simulation of low-concentration sea ice. However, we find a very strong over-estimation in lowconcentration sea ice in the NEMO-CICE model, which is forced by a reanalysis atmosphere and so should have somewhat realistic winds. The dynamical response of sea ice to winds at the edge of the ice may be poorly represented, as we would expect sea ice dynamics to be floe-size dependent. Alternative rheologies (such as a granular rheology, e.g. Feltham 2005) may be better suited to this domain. Concentrations could also be reduced by mechanical interactions between floes. However, we cannot test the impact of such floe-size dependent processes without access to sea ice models that include them. 
The impact of increased floe size, on the other hand, is much smaller (Fig. 3.9c,f). Differences in sea ice concentration between the standard model and the large floe simulation are barely perceptible. Changes in the ice errors relative to the standard model are of the opposite sign compared to the small floe simulation, but these changes are unlikely to be significant. Examining the basal and lateral melt rates, we find that the hemispheric average DJF 1992-2014 mean lateral melt rate accounts for only $5 \%$ of the combined basal and lateral melt rates in the standard model. It accounts for a larger proportion (17 $\%)$ of melt in the Arctic. Decreasing floe diameter by two orders of magnitude increases the lateral melt rate to $83 \%$ of the combined basal and lateral melt. This compensation effect of reduced basal melt when lateral melt is increased was also noted by Tsamados et al. (2015) in the Arctic. On the other hand, increasing the floe diameter by two orders of magnitude effectively switches off lateral melt ( $0.2 \%$ of combined basal and lateral melt). In the latter case, more melting potential is made available for basal melting, which, because Antarctic sea ice is so thin, has the same impact on sea ice concentration as lateral melt. We conclude that there must be alternative reasons for the consistent underestimation of compact summer ice.

Looking at the regional distribution of DJF (the season where the bias is apparent in Fig. 3.5) seasonal mean sea ice concentration averaged over 1992-2014, highconcentration (90-100 \%) ice appears in the observations mean only in the Weddell Sea (Fig. 3.3). Taking the difference between the high-concentration ice in each observational product and the sea ice concentration in the CMIP5 model simulations shows that very few of the models simulate high enough concentrations in this area. Fig. 3.10 shows the difference between the ASI-SSMI observations and the CMIP5 models; differences are slightly enhanced using Bootstrap and less pronounced when using NASA Team. This demonstrates a consistent model tendency to underestimate concentrations in the Weddell Sea, the largest region of multi-year ice in Antarctica. The bias is not present in other seasons, suggesting it is related to overestimated melt or break-up processes, including misrepresentation of sea ice dynamics. 

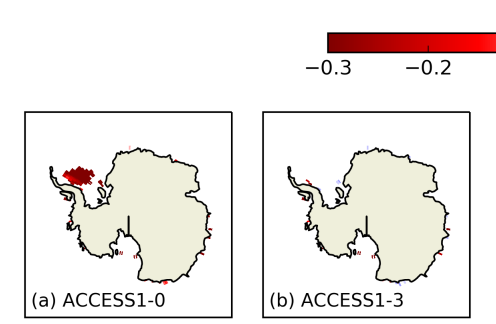

Sea ice concentration
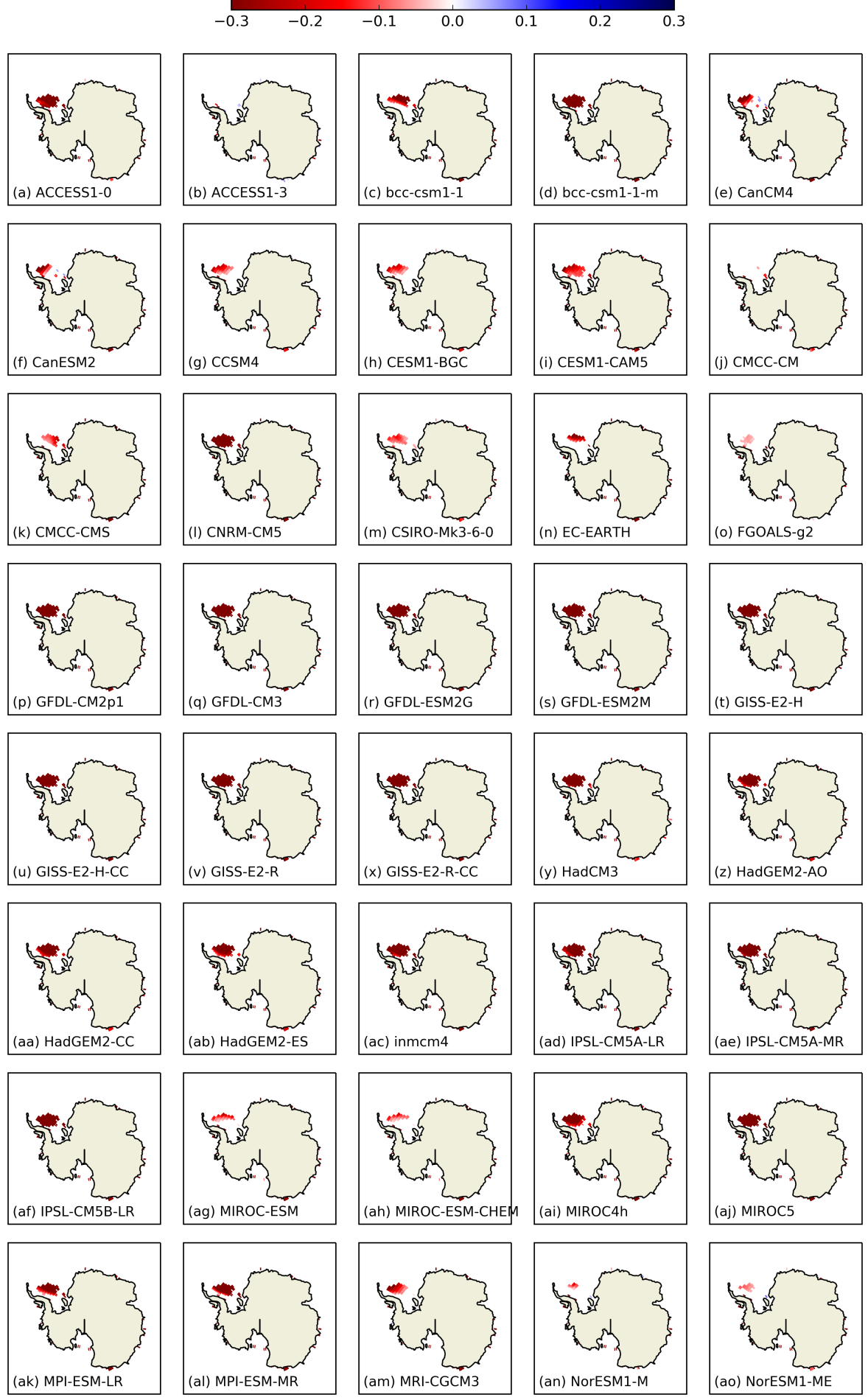

Figure 3.10: Simulation minus observed ASI-SSMI sea ice concentration for DJF 1992-2014 for each CMIP5 model, where only grid cells with observational mean sea ice concentration is $\geq 90 \%$ are considered. Differences are only shown if they are statistically different according to a Student's t-test over $1992-2014(p<5 \%)$. 
Overestimated melt or break-up could be a result of the sea ice model or a biased warm atmosphere or ocean. While consideration of normalized sea ice concentration distribution is intended to remove overall biases caused by (for example) a warm ocean, in summer the warm ocean could shift the whole distribution to lower concentrations. Alternatively, or likely in conjunction with this, regionally important processes may be being misrepresented. Evaluating the ORCA2-LIM coupled ocean-sea ice model, Timmermann (2004) found that overestimation of westerly winds led to an underestimation of sea ice coverage on the eastern side of the Antarctic peninsula, in the Weddell Sea. Other CMIP5 models may simulate high drift speeds due to winds or sea ice rheology, which Lecomte et al. (2016) found correlated with a faster sea ice retreat.

\subsection{Discussion}

In this study, we examine the distribution of sea ice concentration from both models and observations. Firstly, we show that observed sea ice concentration values can differ significantly between three widely-used algorithms for satellite data. This observational uncertainty provides a limit beyond which we cannot further evaluate model agreement with observations. Many sea ice model-observations comparisons use only one satellite dataset assumed to represent the true observed state, an approach which may be sufficient when using sea ice extent, a metric where the various algorithms broadly agree. However, when using metrics that go beyond sea ice extent, using for example sea ice area or sea ice concentration distributions, model evaluation studies should account for the observational range.

We find that simulation of high-concentration $(90 \%+)$ sea ice in models is in better agreement with the NASA Team observations than the observational range including the Bootstrap and ASI-SSMI observations, in agreement with Ivanova et al. (2016), who only examined the CMIP5 multi-model mean. Accounting for the range in three observational products, we find that models overestimate the extent of low-concentration sea ice throughout the year, while underestimating the extent of high-concentration sea ice in summer. This common behaviour across diverse models with varying physics is a result not previously highlighted and warrants further attention. 
We note that using the observational range as an uncertainty estimate neglects biases that are common to the three different satellite observations. As mentioned above, sea ice concentrations are considered to be most uncertain during melt conditions, for large fractions of thin ice and at low concentrations during storms. In the context of the results from the model-observation comparison for normalized sea ice concentration distributions, we suggest that the impact of uncertainty of melt conditions is limited as the high bias in low-concentration ice from CMIP5 models is visible throughout the year. The low bias in high-concentration ice during the melt season would be larger if observations were underestimating ice concentrations in this season. Inclusion of both NASA Team and Bootstrap algorithms, with the former tending to cancel out physical temperature effects, will sample some of this uncertainty.

The underestimation of sea ice concentrations in areas of thin ice $(<35 \mathrm{~cm})$ (Ivanova et al., 2015) may cause a bias at any concentration in the observed normalized sea ice concentration distribution from observations, with the possibility of a positive bias in the very lowest concentrations. Stormy conditions near the ice edge lead to false sea ice concentrations near the ice edge; weather filters may accurately remove these, leave them uncorrected (Andersen et al., 2006) or erroneously remove real sea ice. The latter may underestimate low concentrations. Spreen et al. (2008) suggest the filter method used in ASI-SSMI observations may result in a positive bias in the marginal ice zone, and Steffen and Schweiger (1991) found that the NASA Team algorithm overestimates lowconcentration ice when compared to Landsat imagery. Considering all this evidence we suggest that the magnitude or sign of any systematic biases in satellite radiometer observations is unclear when comparing with climate models. This is particularly true for low concentrations. Here the use of different approaches to weather filters within the different algorithms may assist in sampling observational uncertainty. Development of sea ice satellite emulators, which use climate model output to calculate brightness temperatures (e.g. Tonboe et al. 2011), may help to reduce uncertainty when comparing models to observations in the future.

Categorising models according to whether they explicitly represent lateral melting, which is the only thermodynamic sea ice process that reduces concentrations in models regardless of sea ice thickness, we find a strong impact of this process on low-concentration sea ice. In Subsect. 3.3.4 we briefly review typical sea ice model thermodynamics, and in 
particular the change in concentration induced by lateral melt rate for a region containing floes of a single diameter, which follows Steele (1992). Horvat et al. (2016) finds that development of ocean eddies due to lateral density gradients could induce much larger lateral melt than that suggested from the Steele (1992) geometric model. This would support increasing the lateral melt rate in models, as we have done artificially here through a reduced constant floe size. Heat budget analysis (Nihashi and Ohshima, 2001) and modelling studies (Fichefet and Maqueda, 1997; Ohshima and Nihashi, 2005) suggest that the major cause of Antarctic sea ice decay is atmospheric heat input to open water, which causes bottom and lateral melt. Fichefet and Maqueda (1997) find that sea ice melt by open water plays a larger role in the Antarctic than in the Arctic. We further note that the coefficients in the lateral melt rate used in CICE were measured in the Arctic only (Maykut and Perovich, 1987) and few, if any, observational studies exist on the relative importance of bottom and lateral melt in the Antarctic.

The impacts of enhancing lateral melt via reducing a constant floe size shown here suggest that this process should not be applied in the same way throughout the ice pack. While not all models include such a lateral melt parametrization, the biases at the tails of the concentration distributions from the CMIP5 models point to inclusion of model processes that are not suitable for both high-concentration and low-concentration regimes. A possible conclusion, therefore, is that physics in sea ice models are not heterogeneous enough to represent observed sea ice cover. Given the possible contribution of dynamic processes to model biases in the sea ice concentration distribution, a full exploration of sea ice dynamics for all CMIP5 models using the sea ice concentration budget decomposition of Uotila et al. (2014) would be welcome. Including information on the floe size distribution and floe size dependent processes (e.g. Horvat and Tziperman 2015; Zhang et al. 2016; Bennetts et al. 2014) could improve consistency with observations in the metrics presented here. 


\section{Article citation}

Roach, L. A., S. M. Dean, and J. A. Renwick (2018a), Consistent biases in Antarctic sea ice concentration simulated by climate models, The Cryosphere, 12(1), 365-383, doi:10.5194/tc-12-365-2018

\section{Acknowledgements}

The authors wish to thank Erik Behrens and Jonny Williams for assistance setting up and running the coupled ocean-sea ice model, Stephen Stuart for assistance with CMIP5 output acquisition and Cecilia Bitz for helpful discussions in the early stages of this work. We are grateful to the reviewers, whose comments significantly improved the manuscript. This research was funded via Marsden contract VUW-1408. We acknowledge the World Climate Research Programme's Working Group on Coupled Modelling, which is responsible for CMIP, and we thank the climate modelling groups (listed in Table 3.1) for producing and making available their model output. For CMIP the U.S. Department of Energy's Program for Climate Model Diagnosis and Intercomparison provides coordinating support and led development of software infrastructure in partnership with the Global Organization for Earth System Science Portals. See http: / / cmip-pcmdi. I ln l.gov/ cmip5/data_portal.html for CMIP5 data, http: / / icdc.cen.uni-hamburg.de/ 1/daten/cryosphere/seaiceconcentration-asi-ssmi.html for ASI-SSMI data and https://nsidc.org/data/docs/noaa/g02202_ice_conc_cdr/for the Bootstrap and NASA Team data. NEMO-CICE model output is available from the author upon request. The authors also wish to acknowledge the contribution of NeSI high-performance computing facilities to the results of this research. NZ's national facilities are provided by the NZ eScience Infrastructure and funded jointly by NeSI's collaborator institutions and through the Ministry of Business, Innovation \& Employment's Research Infrastructure programme. 


\section{Chapter 4}

\section{An emergent sea ice floe size distribution in a global coupled ocean-sea ice model}

Findings in the previous chapter suggested that sea ice floe size should be simulated prognostically to improve model representation of lateral melt and therefore simulation of sea ice concentration. In this chapter, published as Roach et al. (2018b), we present a new model for simulation of sea ice floe sizes, which evolve under different physical processes. This is the first global ocean-sea ice model to prognostically simulate a subgrid-scale sea ice floe size distribution.

\subsection{Abstract}

Sea ice is composed of discrete floes, which range in size across orders of magnitude. Here, we present a model that represents the joint distribution of sea ice thickness and floe size. Unlike previous studies, we do not impose a particular form on the sub-gridscale floe size distribution. Floe sizes are determined prognostically by the interaction of five key physical processes: new ice formation, welding of floes in freezing conditions, lateral growth and melt, and fracture of floes by ocean surface waves. Coupled model results suggest that these processes capture first-order characteristics of the floe size distribution, including decay in the distribution with increasing floe size and basin-wide spatial variability in representative radius. Lateral melt and floe welding are particularly important, with wave fracture creating floes at preferred sizes. The addition of floe size 
dependence to the existing model physics results in significant reductions in sea ice concentration, particularly in summer and principally due to floe-size-dependent lateral melt. The increased lateral melt alters partitioning of the melting potential, which reduces basal melt and increases sea ice thickness in some locations. These results suggest that including a floe size distribution may be important for accurate simulation of the polar climate system.

\subsection{Introduction}

The Earth's sea ice cover is a heterogeneous and variable medium, comprised of myriad individual solid pieces, called floes, each identifiable with a horizontal size. Sizes of individual floes vary over an extremely broad range, from centimeters to hundreds of kilometers. The floe size distribution (FSD), $F(r)$, is a probability function that characterizes this variability (Rothrock and Thorndike, 1984). Over a region of the ice-covered ocean, $F(r) \mathrm{d} r$ is the fraction of a region covered by floes with a size between $r$ and $r+\mathrm{d} r$. Floe size has an important relationship with simulated sea ice evolution (Steele, 1992; Horvat et al., 2016; Rynders et al., 2016), which may be particularly relevant for the largely seasonal Antarctic sea ice cover, and as the Arctic ocean transitions from a perennial sea ice cover to a seasonal one (Aksenov et al., 2017).

Current sea ice models are complex, incorporating multiple vertical layers in the snow and ice through which radiation scatters, variable surface treatments such as snow cover and melt ponds, and visco-plastic or elastic-brittle material properties that affect the ice deformation into ridges. Most describe the time evolution of ice using a probability distribution of ice in thickness categories following Thorndike et al. (1975). To date no modern global climate models simulate floe size or the FSD. Recently, pan-Arctic (Zhang et al., 2016) and stand-alone (Bennetts et al., 2017) models which include floe size information have been demonstrated, but these impose the FSD shape or behaviour rather than allowing it to emerge from physical processes acting on individual floes. Further, the power-law FSD profiles used to develop these empirical parametrizations may be inconsistent with observations (Herman, 2010) and the physics of sea ice floes (Horvat and Tziperman, 2017; Herman et al., 2018).

In this study, we allow the FSD to emerge from the interaction of a set of coupled pro- 
cesses, rather than imposing a particular distributional shape. Building from the model of the joint floe size and thickness distribution (FSTD) developed by Horvat and Tziperman $(2015,2017)$, we present the first global ocean-sea ice model to prognostically simulate the sea ice FSD. The scheme is compatible with existing sea ice thickness distribution models and is implemented within the Los Alamos sea ice model, CICE5.1 (Hunke et al., 2015). The model simulates the statistical evolution of floes subject to lateral growth and melt, welding of floes in freezing conditions, new ice formation and fracture of floes by ocean surface waves, with the shape of the FSD emerging from these processes. Using the model in coupled ocean-sea ice simulations, we examine the contribution of those processes to FSD evolution at a hemispheric scale. We further show that including floe size information has a significant impact on sea ice concentration and thickness globally.

This paper proceeds as follows: we discuss the incorporation of a prognostic FSD into CICE in Sec. 4.3. We show results from coupled simulations in Sec. 4.4; discuss limitations, compare to other studies, and make recommendations for observations that would advance FSD models in Sec. 4.5; and conclude in Sec. 4.6.

\subsection{Model}

\subsubsection{Standard model}

The FSD model is implemented as a component of the Los Alamos sea ice model, CICE5.1 (Hunke et al., 2015). CICE ordinarily simulates an ice-thickness distribution (ITD), $g(h)$ (units $\mathrm{m}^{-1}$ ), where $g(h) \mathrm{d} h$ is defined as the fraction of ocean surface covered by ice with thickness between $h$ and $h+\mathrm{d} h$, such that

$$
\int_{0}^{h_{\max }} g(h) \mathrm{d} h=1 .
$$

The sea ice concentration, $c$, is obtained by integrating over all non-zero thicknesses resolved,

$$
\int_{h_{\min }}^{h_{\max }} g(h) \mathrm{d} h=c,
$$


where $h_{\min }$ is the lower bound of the smallest ice thickness class resolved. The sea ice concentration, $c$, and the open water fraction, $\phi$, sum to unity. The evolution of the ITD is,

$$
\frac{\partial g}{\partial t}=-\frac{\partial}{\partial t}(\mu g)-\nabla \cdot(g \mathbf{v})+\psi
$$

where terms on the right hand side, respectively, represent the change in thickness due to thermodynamic growth/melt at a melting/freezing rate $\mu(h)$; advection of the ice thickness distribution by sea ice dynamics at ice velocity $\mathbf{v}$; and redistribution of ice between thickness categories caused by sea ice deformation, $\psi$. We briefly describe the treatment of sea ice thermodynamics in CICE here.

The heat available in the surface ocean to melt or freeze sea ice is denoted $F_{\text {frz/mlt }}$ (units $\mathrm{W} / \mathrm{m}^{2}$ ), and when $F_{\text {frz/mlt }}<0$, the sea ice melts. Ice thickness changes at the ice base are determined by balancing the conductive heat flux at the bottom surface, $F_{c b}$, and the net downward heat flux from the ice to the ocean, $F_{\text {bot }}$ (Maykut and McPhee, 1995),

$$
F_{\mathrm{bot}}=-c_{p}^{\mathrm{ocn}} \rho_{w} C_{h} u_{*}\left(T_{w}-T_{f}\right),
$$

where $c_{p}^{\text {ocn }}$ and $\rho_{w}$ are the ocean heat capacity and density, $C_{h}$ is a heat transfer coefficient, $u_{*}$ is the ocean-ice friction velocity, $T_{f}$ is the freezing temperature and $T_{w}$ is the ocean surface temperature.

Lateral sea ice melting is obtained as a function of a fixed floe size parameter, $L$. CICE uses a single floe size of $L \equiv 300 \mathrm{~m}$, which is an order of magnitude larger than the scale at which lateral melting is believed to affect sea ice volume evolution (Steele, 1992). The change in sea ice concentration due to lateral melt follows Steele (1992),

$$
\frac{\mathrm{d} g(h)}{\mathrm{d} t}=\frac{g(h)}{L} w_{\mathrm{lat}},
$$

with a vertically-averaged lateral melt rate, $w_{\text {lat }}$, that is assumed to be uniform around the perimeter of each floe, given by Josberger and Martin (1981),

$$
w_{\text {lat }}=m_{1}\left(T_{w}-T_{f}\right)^{m_{2}}
$$

The coefficients $m_{1}$ and $m_{2}$ are the best fit to data quoted by Maykut and Perovich (1987), measured in a single static lead in the Canadian Arctic archipelago over a three week pe- 
riod. The sum of $F_{\text {bot }}$ and the heat required to effect the change in concentration due to lateral melt, $F_{\text {side }}$, cannot exceed the melting potential, $F_{\text {frz/mlt }}$, and are reduced proportionally if this occurs.

During freezing conditions, when $F_{\text {frz/mlt }} \geq 0$, a volume of sea ice, $V_{\text {new }}$, is produced in proportion to $F_{\text {frz/mlt }}$. This volume is added to the thinnest category, provided $V_{\text {new }} / \phi \leq 0.9 h_{1}^{\prime}$, where $h_{1}^{\prime}$ is the upper boundary of the thinnest category. The fractional coverage of the thinnest category is increased by $\min \left(\phi, V_{\text {new }} / 0.05 \mathrm{~m}\right)$. However, if $V_{\text {new }} / \phi>0.9 h_{1}^{\prime}$, then a volume $0.9 h_{1}^{\prime} \phi$ is added to the thinnest category and its fractional coverage is raised by $\phi$, and the surplus volume $V_{\text {new }}-0.9 h_{1}^{\prime} \phi$ is distributed to all other thickness categories in proportion to their fractional coverage.

\subsubsection{The joint floe size and ice thickness distribution}

We extend the definition of the ice-thickness distribution following Horvat and Tziperman (2015) to a joint floe size and thickness distribution (FSTD). Individual floes are identified with a size $r$ and area $x(r)$, where $x(r)=4 \alpha r^{2}$ for $\alpha=0.66<\pi / 4$ (Rothrock and Thorndike, 1984). The probability distribution $f(r, h) \mathrm{d} r \mathrm{~d} h$ is the fraction of grid surface area covered by ice with thickness between $h$ and $h+\mathrm{d} h$ and lateral floe size between $r$ and $r+\mathrm{d} r$. The FSTD satisfies

$$
\int_{r_{\min }}^{r_{\max }} \int_{0}^{h_{\max }} f(r, h) \mathrm{d} r \mathrm{~d} h \equiv \int_{\mathcal{R}} \int_{\mathcal{H}} f(r, h) \mathrm{d} r \mathrm{~d} h=1 .
$$

Integrating the FSTD over all floe sizes yields the ITD,

$$
\int_{\mathcal{R}} f(r, h) \mathrm{d} r=g(h),
$$

whereas integrating the FSTD over all ice thicknesses gives the FSD, $F(r)$,

$$
\int_{\mathcal{H}} f(r, h) \mathrm{d} h=F(r) .
$$

We can also define the number FSTD, $f^{N}(r, h)$, where $f^{N}(r, h) \mathrm{d} r \mathrm{~d} h$ is the number of floes per unit ocean surface area with thickness between $h$ and $h+\mathrm{d} h$ and lateral floe 
size between $r$ and $r+\mathrm{d} r$,

$$
f^{N}(r, h)=\frac{f(r, h)}{4 \alpha r^{2}} .
$$

The number FSD, obtained by integrating $f^{N}(r, h) \mathrm{d} h$ over all ice thicknesses, is often used in observational studies (e.g. Perovich and Jones 2014).

Following Horvat and Tziperman (2015), time evolution of the FSTD is given by

$$
\frac{\partial f(r, h)}{\partial t}=-\nabla \cdot(f(r, h) \mathbf{v})+\mathcal{L}_{T}+\mathcal{L}_{M}+\mathcal{L}_{W}
$$

The terms on the right hand side represent forcing of the distribution $f(r, h)$ by advection, thermodynamics, mechanical interactions between floes (ridging and rafting), and fracture by ocean surface waves, respectively.

To implement this model in CICE, we define a modified areal FSTD (mFSTD), $L(r, h)$, where, within a given thickness range between $h$ and $h+\mathrm{d} h, L(r, h) \mathrm{d} r$ is the fraction of ice with lateral floe size between $r$ and $r+\mathrm{d} r$. By definition, this satisfies

$$
\int_{\mathcal{R}} L(r, h) \mathrm{d} r=1
$$

and

$$
f(r, h)=g(h) L(r, h) .
$$

Implementation of the mFSTD allows preservation of the standard model formulation for the ITD.

We neglect the two-way relationship between floe size and mechanical redistribution, retaining the standard CICE scheme for mechanical redistribution used to evolve the ITD. Mechanical redistribution reduces the area fractions of all floes equally, without affecting the mFSTD. Transport of the FSTD is achieved using the standard CICE scheme for tracer advection. The sizes of floes do not appear directly in any terms in the momentum equation or constitutive law. In reality, we would expect floe sizes to affect both mechanical redistribution and transport of the FSTD, but the precise relationships are uncertain and we assume that they are of second-order importance to simulation of the FSD.

Apart from advection, the processes which determine the FSTD are thermodynamics- 
lateral melt and growth, freezing-together of floes, and new ice formation-and mechanical wave fracture. These are described in more detail below.

\subsubsection{Thermodynamics}

Thermodynamic changes to the FSTD are given by

$$
\mathcal{L}_{T}(r, h)=-\nabla_{(r, h)} \cdot(f(r, h) \mathbf{G})+\frac{2}{r} f(r, h) G_{r}+\delta\left(r-r_{\text {min }}\right) \delta\left(h-h_{\text {min }}\right) \dot{A}_{p}+\beta_{\text {weld }} .
$$

The first two terms on the right-hand side in Eqn. 4.14 represent growth and melt of existing floes in thickness and lateral size, at a rate $\mathbf{G}=\left(G_{r}, G_{h}\right)$. The third term represents

growth of new ice: new floes are created at a rate $\dot{A}_{p}$ in the smallest thickness category $h_{\min }$, and the smallest lateral size category $r_{\min }$, i.e. that all ice forms initially as pancakes. To allow for the joining of individual floes to one another, we represent the welding together of floes in freezing conditions via the fourth term, $\beta_{\text {weld }}$.

In melting conditions, the lateral melt rate is

$$
G_{r}=w_{\text {lat }}
$$

to preserve consistency with the standard model, with $w_{\text {lat }}$ determined via Eqn. 4.6.

In freezing conditions, the lateral growth rate is

$$
G_{r}=A_{\text {lat }} V_{\text {new }} / \Delta t
$$

where $\Delta t$ is the time step and $V_{\text {new }}$ is the volume of new ice growth in $\Delta t$, as per the standard model. $A_{\text {lat }}$ is the fraction of new ice growth that is taken to adhere to floe edges, representing lateral growth of existing floes. This is related to the 'lead region', the area comprised of all annuli of width $r_{l w}$ (Table 4.1) around floes. The fraction of the domain belonging to the lead region, $\phi_{\text {lead }}$, is

$$
\phi_{\text {lead }}=\min \left[\int_{\mathcal{R}} \int_{\mathcal{H}} f(r, h)\left(\frac{2 r_{l w}}{r}+\frac{r_{l w}^{2}}{r^{2}}\right) \mathrm{d} r \mathrm{~d} h, \phi\right],
$$

where $\phi$ is the open water fraction. Noting that the circumference of a floe is $4 \alpha \cdot 2 r$, the 
total lateral surface area of floes, per unit area of the ocean surface, is,

$$
\overline{2 h r}=\int_{\mathcal{R}} \int_{\mathcal{H}} f^{N}(r, h) 8 \alpha r h \mathrm{~d} r \mathrm{~d} h .
$$

Then the fraction of new ice growth adhering to floe edges, $A_{\text {lat }}$, is the product of the lead region with the fractional contribution of lateral surface area to the total surface area,

$$
A_{\text {lat }}=\phi_{\text {lead }} \frac{\overline{2 h r}}{\overline{2 h r}+c} .
$$

The volume that remains after lateral growth, $\left(1-A_{\text {lat }}\right) V_{\text {new }}$, is distributed according to the standard CICE new ice growth formulation as described in Subsec. 4.3.1. We choose to place newly formed ice in the smallest floe size category, parametrizing them as pancake floes, as mentioned above. See Sec. 4.5 for discussion of this choice.

Floes that are determined to be in contact with one another while the upper ocean is being cooled may freeze together (Shen and Ackley, 1991), a process that is dominant in the Southern Ocean (Wadhams et al., 1987). We consider sea ice floes randomly placed on the model domain and allow them to weld together thermodynamically during freezing conditions according to the probability that they overlap. For simplicity, we briefly change variables to floe area $x=4 \alpha r^{2}$ defined on $\mathcal{X}=\left[x_{\min }, x_{\max }\right]$, and presume sea ice is all of the same thickness. We define the area number density function $N(x)$ (units $\mathrm{m}^{-4}$ ), with $N(x) \mathrm{d} x$ equal to the number of floes per unit area between floe area $x$ and $x+\mathrm{d} x$, noting that the area fraction occupied by floes with area between $x$ and $x+\mathrm{d} x$ is $x$. $N(x) \mathrm{d} x$. The geometric probability of overlap is described using a 'coagulation equation' (Smoluchowski, 1916; Filbet and Laurençot, 2004),

$$
\frac{\partial N(x, t)}{\partial t}=\frac{1}{2} \int_{\mathcal{X}} K\left(x^{\prime}, x-x^{\prime}\right) \mathrm{d} x^{\prime}-\int_{\mathcal{X}} K\left(x, x^{\prime}\right) \mathrm{d} x^{\prime} .
$$

$K(a, b)$ (units $\mathrm{m}^{-6} s^{-1}$ ) is the 'coagulation kernel', where $K(a, b) \mathrm{d} a \mathrm{~d} b \mathrm{~d} t$ is the number of mergers per unit area of floes with area between $a$ and $a+\mathrm{d} a$, and $b$ and $b+\mathrm{d} b$ over a period $\mathrm{d} t$, and $K(a, b) \equiv 0$ for any $a, b \leq 0$. The first integral in Eqn. 4.18 accounts for the formation of floes of area $x$ resulting from the merger of two floes with respective areas $x^{\prime}$ and $x-x^{\prime}$, where $x^{\prime}<x$. The second integral describes the loss of floes with area $x$ by coagulation with other floes. We compute the coagulation kernel $K\left(x, x^{\prime}\right)$ as 
the product of the area fraction of floes of size $x$ and $x^{\prime}$,

$$
K\left(x, x^{\prime}\right)=\kappa \cdot x \cdot x^{\prime} \cdot N(x) N\left(x^{\prime}\right)
$$

where $\kappa$ is a rate per unit area. Integrating Eqn. 4.18 over all $x$ leads to the time change of floe number per unit area, $\mathcal{N}$,

$$
\begin{aligned}
\frac{\partial \mathcal{N}}{\partial t} & =\kappa\left[\int_{\mathcal{X}} \mathrm{d} x \int_{\mathcal{X}}\left(\frac{1}{2} x^{\prime}\left(x-x^{\prime}\right) N\left(x^{\prime}\right) N\left(x-x^{\prime}\right)-x x^{\prime} N(x) N\left(x^{\prime}\right)\right) \mathrm{d} x^{\prime}\right] \\
& =\frac{\kappa}{2}\left[\int_{x_{\min }}^{x} \mathrm{~d} x^{\prime}\left(\int_{\mathcal{X}} x^{\prime}\left(x-x^{\prime}\right) N\left(x^{\prime}\right) N\left(x-x^{\prime}\right) \mathrm{d} x\right)-2 c^{2}\right] \\
& =-\frac{\kappa}{2} c^{2},
\end{aligned}
$$

where we make use of the fact that $\int x N(x) \mathrm{d} x=c$. The rate per unit area $\kappa$ is the total number of floes that weld with another, per square meter, per unit time, in the case of a fully covered ice surface $(c=1)$, equal to twice the reduction in total floe number. Roach et al. (2018c) found a lower bound on $\kappa$ of $0.001 \mathrm{~m}^{-2} \mathrm{~s}^{-1}$ in observations of small floes freezing together in the autumn Arctic Ocean. We use a value of $\kappa=0.01 \mathrm{~m}^{-2} \mathrm{~s}^{-1}$ for the floe welding parameter.

\begin{tabular}{llll}
\hline Parameter & Description & Value & Reference \\
\hline$\alpha$ & Non-circularity of floes & 0.66 & Rothrock and Thorndike (1984) \\
$r_{l w}$ & Width of lead region & $r_{1}$ & Horvat and Tziperman (2015) \\
$\kappa$ & Rate constant for merging & $0.01 \mathrm{~m}^{-2} \mathrm{~s}^{-1}$ & Roach et al. (2018c) and see Appendix A \\
$\epsilon_{\text {crit }}$ & Critical strain & $3 \times 10^{-5}$ & $\begin{array}{l}\text { Horvat and Tziperman (2015), Kohout } \\
\text { and Meylan (2008) }\end{array}$ \\
& & & Toyota et al. (2011) \\
$t_{\text {wave }}$ & Smallest floe size affected & $10 \mathrm{~m}$ & \\
& by waves & & \\
\hline
\end{tabular}

Table 4.1: Parameters that are not present in standard CICE. $r_{1}$ denotes the smallest floe size resolved in the model 


\subsubsection{Wave fracture}

Following Horvat and Tziperman (2015), the change in the FSTD $f(r, h)$, per unit time due to fracture by ocean surface waves is,

$$
\mathcal{L}_{W}(r, h)=-\Omega(r, h)+\int_{\mathcal{R}} \int_{\mathcal{H}} \Omega\left(s, h_{s}\right) \zeta\left(r, h, s, h_{s}\right) \mathrm{d} s \mathrm{~d} h_{s}
$$

$\Omega(r, h) \mathrm{d} r \mathrm{~d} h$ is the fraction of ocean surface area covered by floes with size and thickness between $(r, h)$ and $(r+\mathrm{d} r, h+\mathrm{d} h)$ that is fractured by waves per unit time. $\zeta\left(r, h, s, h_{s}\right) \mathrm{d} r \mathrm{~d} h$ is the fraction of ocean surface area covered by floes with size and thickness between $(r, h)$ and $(r+\mathrm{d} r, h+\mathrm{d} h)$ formed due to the fracture of floes with size and thickness between $\left(s, h_{s}\right)$ and $\left(s+\mathrm{d} s, h_{s}+\mathrm{d} h_{s}\right)$. The first term on the right-hand side in Eqn. 4.20 thus represents the fracture of floes at a given size and thickness into smaller sizes, and the second term represents the fracture of floes at larger sizes that result in floes at a given size and thickness.

We proceed by calculating the fractures that would occur if waves enter a fully icecovered region defined in one dimension in the direction of propagation, and then apply the outcome proportionally to the ice-covered fraction in each grid cell. Noting that floe size is half its diameter, the sum of floe sizes in a one-dimensional, fully ice-covered domain is equal to the half the domain length, $D / 2$. We consider the histogram of floe sizes, $W(r)$, formed due to the fracture of sea ice by waves, where $W(r) \mathrm{d} r$ is equal to the number of fractures with a resulting floe size between $r$ and $r+\mathrm{d} r$,

$$
\int_{\mathcal{R}} r W(r) \mathrm{d} r=D / 2
$$

The function $\zeta\left(r, h, s, h_{s}\right)$ is the fraction of $D$ composed of fractures of size $r$, equal to $r W(r)$ if $r<s$, and zero otherwise,

$$
\zeta\left(r, h, s, h_{s}\right)=\frac{r W(r)}{\int_{r_{\min }}^{s} r W(r) \mathrm{d} r} \delta\left(h-h_{s}\right) \Theta(s-r)
$$

where $\Theta$ is the Heaviside step function. By definition,

$$
\int_{\mathcal{R}} \int_{\mathcal{H}} \zeta\left(r, h, s, h_{s}\right) \mathrm{d} r \mathrm{~d} h=1
$$


so Eqn. 4.20 conserves sea ice area and volume. We compute the area of floes of size $(r, h)$ that is fractured per unit time as

$$
\Omega(r, h)=f(r, h) \frac{c_{g}}{D}\left(\frac{1}{D / 2} \int_{r_{\min }}^{r} r^{\prime} W\left(r^{\prime}\right) \mathrm{d} r^{\prime}\right)
$$

the product of three terms: (1) the fraction of ocean surface area originally covered by floes of size $(r, h) ;(2)$ the fraction of the domain that is reached by ocean surface waves moving at their group velocity $c_{g},\left(c_{g} / D\right)$; and (3) the fraction of a fully-ice covered domain of width $D$ that would be fractured into radii smaller than $r$.

It remains to compute the histogram of new floe sizes $W(r)$, for which we require the sea surface height field $\eta(x)$. In the absence of a coupled wave model that simulates wave attenuation in ice, we construct an approximate attenuated sea surface height field using hindcast wave data outside the sea ice region. We neglect swell induced by winds within the ice pack and only draw in ocean swell along lines of constant longitude. For each ice-covered grid cell, we find the closest equatorward non-ice covered grid cell along lines of constant longitude. If this grid cell is land, no wave fracture occurs. If this grid cell is not land, we select the significant wave height and mean period from a wave model hindcast. The ocean wave spectrum is then constructed as a Bretschneider spectrum, following Horvat and Tziperman (2015) and Bennetts et al. (2017). It is attenuated exponentially according to the number of floes in the grid cells between the ice-covered grid cell being considered and the non-ice-covered one. The attenuation coefficient is a quadratic function of sea ice thickness and wave period fit by Horvat and Tziperman (2015) to the results of Kohout and Meylan (2008). Further information can be found in the Supplement of Horvat and Tziperman (2015).

From the local ocean surface wave spectrum we generate a realization of the sea surface height field using a random phase as in Horvat and Tziperman (2015). Assuming that sea ice flexes with the sea surface height field $\eta(y)$, strain $\epsilon$ is given by

$$
\epsilon=\frac{h}{2} \frac{\partial^{2} \eta}{\partial y^{2}}
$$

where $y$ is the spatial coordinate. The derivative is computed between successive extrema of the sea surface height, either (maximum, minimum, maximum) or (minimum, maxi- 
mum, minimum). If the strain between successive extrema exceeds a critical value, $\epsilon_{\text {crit }}$ (Table 4.1), new floes are formed with diameters equal to the distance between the extrema. New floe radii resulting from fracture are collected into a histogram, $W(r)$, which depends only on the local sea surface height field. In the interests of computational expense, $W(r)$ and $c_{g}$ are computed offline for different values of sea ice thickness, mean wave period, significant wave height, and number of attenuating floes. This look-up table defines 5000 attenuated sea surface height fields which can be used to fracture ice during code integration. Given a sea surface height field, the scheme computes the new floe sizes generated by wave fracture explicitly, without requiring any assumptions about the FSD.

\subsection{Results}

The additional physics described in Subsec. 2.2-2.4 has been implemented in CICE5.1 (Hunke et al., 2015) and coupled to the NEMO ocean model, using a configuration based on Rae et al. (2015). The ocean-sea ice model is forced with the atmospheric reanalysis JRA-55 (Japan Meteorological Agency, 2013) and run on a $1^{\circ}$ tripolar grid. All simulations described here use repeated atmospheric forcing from a single year. We choose a pre-satellite era year (1975), as these spin-up simulations will be used to initialize transient simulations over the satellite era in later work. Wave forcing corresponding to the same year is taken from a hindcast of the ocean surface wave model, Wavewatch III (Tolman, 2009), which was also forced by JRA-55.

We present here two experiments: a simulation using the standard model (CICE5.1), and a simulation including a prognostic FSD as described above. All analysis uses monthly model output. Floe size categories follow a Gaussian spacing and span a similar range to those chosen by Zhang et al. (2015). Finite differencing in floe size space follows the scheme used by Hibler (1980) for finite differencing in thickness space.

Parameters which are not present in standard CICE and their values are shown in Table 4.1. As global observations of sea ice FSD are not available, parameter values have not been tuned or calibrated to reproduce certain FSD behaviour and are based on estimates from previous studies. More information on the parameter values and their uncertainty can be found in the references provided in Table 4.1. In particular, Horvat and Tziper- 
man (2015) performed local sensitivity tests for most parameters listed. As Roach et al. (2018c) suggest that their estimated lower bound for the floe welding parameter, $\kappa$, is conservative, we use a value that is one order of magnitude higher. $\kappa$ is the only new parameter presented here, so we include results from an experiment where its value is reduced in Appendix A. Grid-cell average floe sizes depend strongly on this parameter (see Appendix A). Naturally, we expect floe sizes to also depend on the choice of floe size categories. More investigation of parameter sensitivity is required, but should occur in fully-coupled atmosphere-ocean simulations where all feedbacks are included-a step which is beyond the scope of this paper.

A key test of the new model physics is whether a sea ice FSD showing physically reasonable characteristics can be simulated in model experiments that begin without FSD initialization, sea ice cover and imposed FSD shape. All simulations are initialized without sea ice cover. Sea ice volume stabilizes after 15 years in the Arctic and after 45 years in the Antarctic. All further analysis is therefore conducted over the final twenty years of a 65 year model run. While detailed information is simulated at the sub-grid-scale, here we focus on resulting characteristics at the hemispheric scale to give an overall picture of model behaviour without focusing on any particular region. Horvat and Tziperman (2015, 2017) describe behaviour of most processes included here at the sub-grid-scale. We proceed by first describing overall behaviour of simulated floe size and then examining how different processes contribute to it.

To show floe size characteristics spatially at the hemispheric scale, we average over floe sizes. Fig. 4.1 shows the cell-average area-weighted 'representative' floe radius, $r_{a}$, which is defined using the areal FSTD,

$$
r_{a}=\frac{\int_{\mathcal{R}} \int_{\mathcal{H}} r f(r, h) \mathrm{d} r \mathrm{~d} h}{\int_{\mathcal{R}} \int_{\mathcal{H}} f(r, h) \mathrm{d} r \mathrm{~d} h}
$$

The representative floe radius climatology in Fig. 4.1 is obtained after beginning the simulation without sea ice cover and allowing it to spin up. In the Northern Hemisphere $(\mathrm{NH})$, the representative floe radius is largest in the centre of the ice pack and smaller towards the edges at the winter maximum in March (Fig. 4.1a). At the summer minimum in September, there are fewer very small and very large representative radii (Fig. 4.1b). Larger floes are concentrated around the coast near the Canadian archipelago and East 


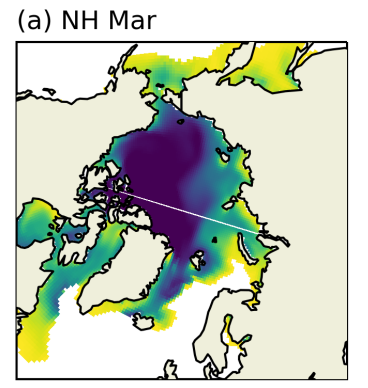

(b) NH Sep

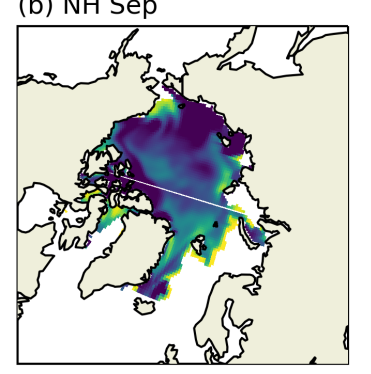

Representative radius $(\mathrm{m})$

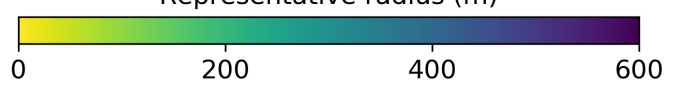

Figure 4.1: The simulated representative floe radius, averaged over twenty years following spin-up, in (a, c) March and (b, d) September, in (a, b) the Northern Hemisphere and (c, d) the Southern Hemisphere. The range displayed is chosen to display both hemispheres on the same scale; dark purple may be greater than $600 \mathrm{~m}$.

Siberian sea. Generally, the representative floe radius is smaller in the Southern Hemisphere (SH) than the NH (Fig. 4.1c-d). In the winter, floes are largest in areas of compact ice, such as the Amundsen and Weddell Seas (Fig. 4.1d). In the summer, large floes are found on the edge of the ice cover (Fig. 4.1c).

Fig. 4.2a-d shows total hemispheric number distributions, which are obtained by integrating $f^{N}(r, h)$ over sea ice thickness and the ocean area in each hemisphere, for the $\mathrm{NH}$ in March (Fig. 4.2a), the NH in September (Fig. 4.2b), the SH in March (Fig. 4.2c) and the SH in September (Fig. 4.2d). The four total number distributions have a similar shape. A high number of small $(<5 \mathrm{~m})$ floes is simulated all year, with more during the winter months than the summer months, due to the production of new pancake ice at the smallest resolved floe size. All four distributions in Fig. 4.2a-d show a significant fraction of floes in the largest floe size category $(>750 \mathrm{~m}$ ), which arises from the truncation of floe size categories. The $\mathrm{SH}$ shows greater seasonal variation than the $\mathrm{NH}$, with an order of magnitude more floes per unit area at nearly all sizes in September (Fig. 4.2d) than in March (Fig. 4.2c). The NH has more very large $(>750 \mathrm{~m})$ floes per unit area than the 

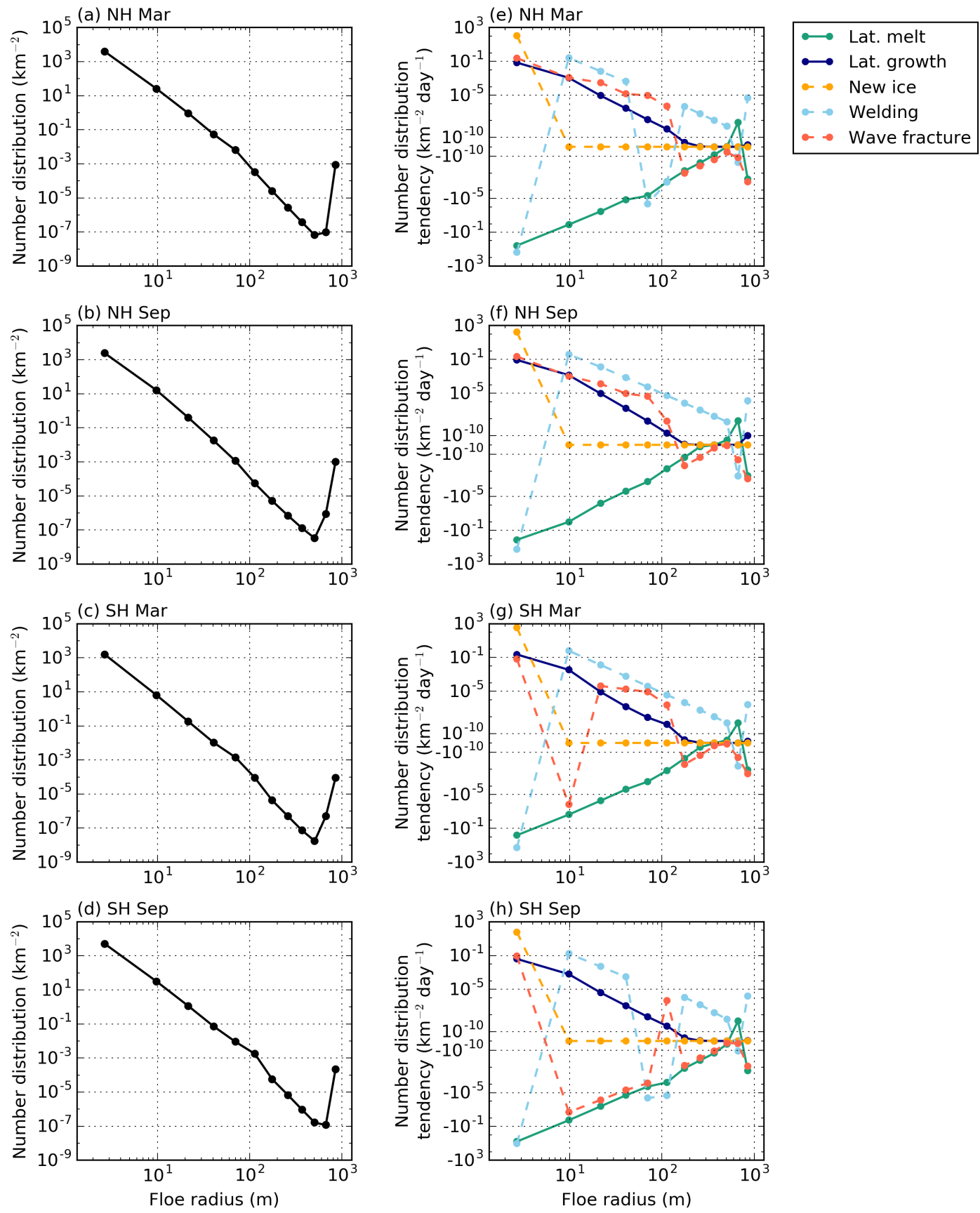

Figure 4.2: (a) The Northern Hemisphere floe number distribution in March averaged over the twenty years following model spin-up. (b-d) Same as (a), for the Northern Hemisphere in September, the Southern Hemisphere in March, and the Southern Hemisphere in September, respectively. (e) The net tendency in the floe number distribution from different physical processes in the Northern Hemisphere in March averaged over the twenty years following model spin-up. The axis in (e) is linearized around zero. (f-h) Same as (e), for the Northern Hemisphere in September, the Southern Hemisphere in March, and the Southern Hemisphere in September respectively. 
SH. Some bending in the distribution is visible at floe sizes of around $100 \mathrm{~m}$, particularly in the SH in September (Fig. 4.2d).

Fig. 4.2e-h show the tendencies arising from different floe processes in the total hemispheric number distributions, where the tendency in the number FSTD due to some process is defined as

$$
\frac{\mathrm{d} f^{N}(r, h)}{\mathrm{d} t}=\frac{1}{\mathrm{~d} t}\left(f(r, h)_{\mathrm{after} \text { process }}^{N}-f(r, h)_{\text {before process }}^{N}\right)
$$

and the model monthly output is the time average of $\frac{\mathrm{d} f(r, h)}{\mathrm{d} t}$. The tendencies at each floe size are the net result of floes being added to and removed from each floe size. Fig. $4.2 \mathrm{f}$ shows the NH in September and illustrates the general tendencies of the different processes. New ice growth creates very small floes; lateral growth and melt respectively act to increase and reduce the number at most sizes; wave fracture redistributes large floes to smaller sizes; and floe welding redistributes all floes to larger sizes.

Floe welding has the largest magnitude tendency of all five processes (Fig. 4.2e-h). Welding moves the smallest floes, created during new ice formation, to larger sizes and is the dominant process in the creation of very large floes. Fracture is a process that destroys large floes and produces smaller floes, so we expect the tendency of floe production to be negative for larger floes. In our simulation, the largest six floe sizes show a net loss due to wave fracture with a shape that is similar to their number distribution (but inverted). Sizes below around $150 \mathrm{~m}$ show a net gain, as large floes fracture into them, driving the bending in the total floe number distribution at this size (Fig. 4.2a-d). Peaks around 100 $m$ induced by wave fracture are balanced out by stronger freezing together of floes at that size in winter (Fig. 4.2e, h). Of the five processes, wave fracture has the most significant hemispheric difference, with net losses at some sizes below $100 \mathrm{~m}$ in the $\mathrm{SH}$, unlike the $\mathrm{NH}$.

Lateral melt is the dominant process to reduce floe sizes (Fig. 4.2e-h). It results in a net gain in the next-to-largest floe size category, due to the large number of floes in the largest floe size category (Fig. 4.2a-d). Lateral melt is around two orders of magnitude more important than lateral growth (Fig. 4.2e-h). Note that the model scheme directly couples lateral growth and new ice formation, such that if a larger portion of new ice went into lateral growth, fewer very small $(<5 \mathrm{~m})$ floes would be created. 
Fig. 4.3a-e show the tendency in representative radius,

$$
\frac{\mathrm{d} r_{a}}{\mathrm{~d} t}=\frac{\int_{\mathcal{R}} \int_{\mathcal{H}} r \frac{\mathrm{d} f(r, h)}{\mathrm{d} t} \mathrm{~d} r \mathrm{~d} h}{\int_{\mathcal{R}} \int_{\mathcal{H}} f(r, h) \mathrm{d} r \mathrm{~d} h},
$$

hemispherically averaged for each process to give a sense of the seasonality of different processes. Wave fracture and lateral melt are much more impactful during the summer months than the rest of the year (Fig. 4.3a, e). During the summer months, there are more small floes (Fig. 4.1b-c), exposing more perimeter to lateral melt and allowing waves to penetrate deeper into the ice field. New ice growth climbs from zero just before the summer minimum and peaks two months after (Nov in the NH, Apr in the SH), gradually decreasing over the other months (Fig. 4.3b). Floe welding is strong all year outside of the summer months (JJA in the NH, DJF in the SH) (Fig. 4.3d).

Fig. 4.3f-o show the spatial variability of different processes, with each subplot showing the month where the net effect of each process is largest (according to Fig. 4.3a-e). For example, Fig. 4.3f shows lateral melt in the NH in July, which Fig. 4.3a shows is the month of largest impact. For all processes, the largest impacts occur around the ice edge (Fig. 4.3f-o). Floe welding is the only size-increasing process to have substantial impacts in the ice interior (Fig. 4.3i, n). It is the dominant driver in the creation of large floes (Fig. 4.2e-h) and thus controls the behaviour of floe sizes in the central ice pack (Fig. 4.1a). Floe size reductions due to wave fracture occur along lines of constant longitude with few impacts in the central ice pack (Fig. 4.3j, o).

Of the five processes that determine the FSTD, only lateral melt, new ice formation and lateral growth directly change sea ice concentration, with lateral growth being the only of these not parametrized in the standard model. Yet even with these similarities to the standard model, the addition of a FSD results in significant changes to the standard model sea ice climatology. Fig. 4.4 shows the sea ice concentration simulated by the standard model and the difference between the standard and FSTD models. Only differences significant at the $95 \%$ confidence level are shown. The inclusion of floe sizes generally acts to lower sea ice concentrations, particularly in already low-concentration areas. The average sea ice concentration reduction for regions that have reductions significant at the $95 \%$ confidence level is $-10 \%$ in September in the $\mathrm{NH}$ and $-40 \%$ in March in the SH. 
At the ice edge, some of these represent total removal of ice in a grid cell. There are also small areas of increased concentrations at a similar magnitude to the decreases, such as the Weddell Sea (Fig. 4.4g). The small areas of increased concentrations tend to be near areas of increased ice advection. Overall, impacts are larger in the SH (Fig. 4.4g, h) than the NH (Fig. 4.4e, f), and in summer months (Fig. 4.4f, g) than winter months (Fig. 4.4e, h).

There are also significant differences when considering sea ice thickness. Fig. 4.5 shows the the grid cell mean thickness, which is the volume of ice per unit area, for the standard model and the difference between the standard and FSTD models. There are both increases and decreases in sea ice thickness relative to the standard model. The average reduction in the thickness of the ice-covered portion of grid cells (for regions that have reductions significant at the $95 \%$ confidence level $)$ is $-5 \%(10 \mathrm{~cm})$ in September in the $\mathrm{NH}$ and $-12 \%(13 \mathrm{~cm})$ in March in the $\mathrm{SH}$. Likewise, the average increase is $6 \%$ (13 $\mathrm{cm})$ in September in the $\mathrm{NH}$ and $25 \%(23 \mathrm{~cm})$ in March in the SH. Maximum increases and reductions in thickness are much greater at some locations.

Differences in lateral melt rates between the standard and FSTD models, which occur via the replacement of $L=300 \mathrm{~m}$ in Eqn. 4.5 with the distribution-integrated factor from Eqn. 4.14, are near-universally positive and coincide with areas of concentration decrease. Summing hemispherically, the total lateral melt rate increases approximately threefold in both hemispheres relative to the standard model (in September in the $\mathrm{NH}$ and March in the SH). This acts to reduce concentrations, and also allows some areas of increased frazil growth into the new open water.

Drawing a larger heat flux to melt ice laterally from the oceanic melting potential means that less is available for basal melt, which may contribute to thicker ice. The total hemispheric basal melt rate decreases by $20 \%$ and $30 \%$ in the $\mathrm{NH}$ in March and the $\mathrm{SH}$ in September respectively relative to the standard model. This reduction in basal melt occurs principally in areas of thick ice, where there is not much melting potential available to divide between basal and lateral melt. Therefore, areas of thick ice experience less basal melt in the FSTD model compared to the standard model, and so remain thicker throughout the year compared to the standard model. Areas of thin ice, where there is a higher melting potential, do not experience this basal melt reduction. 

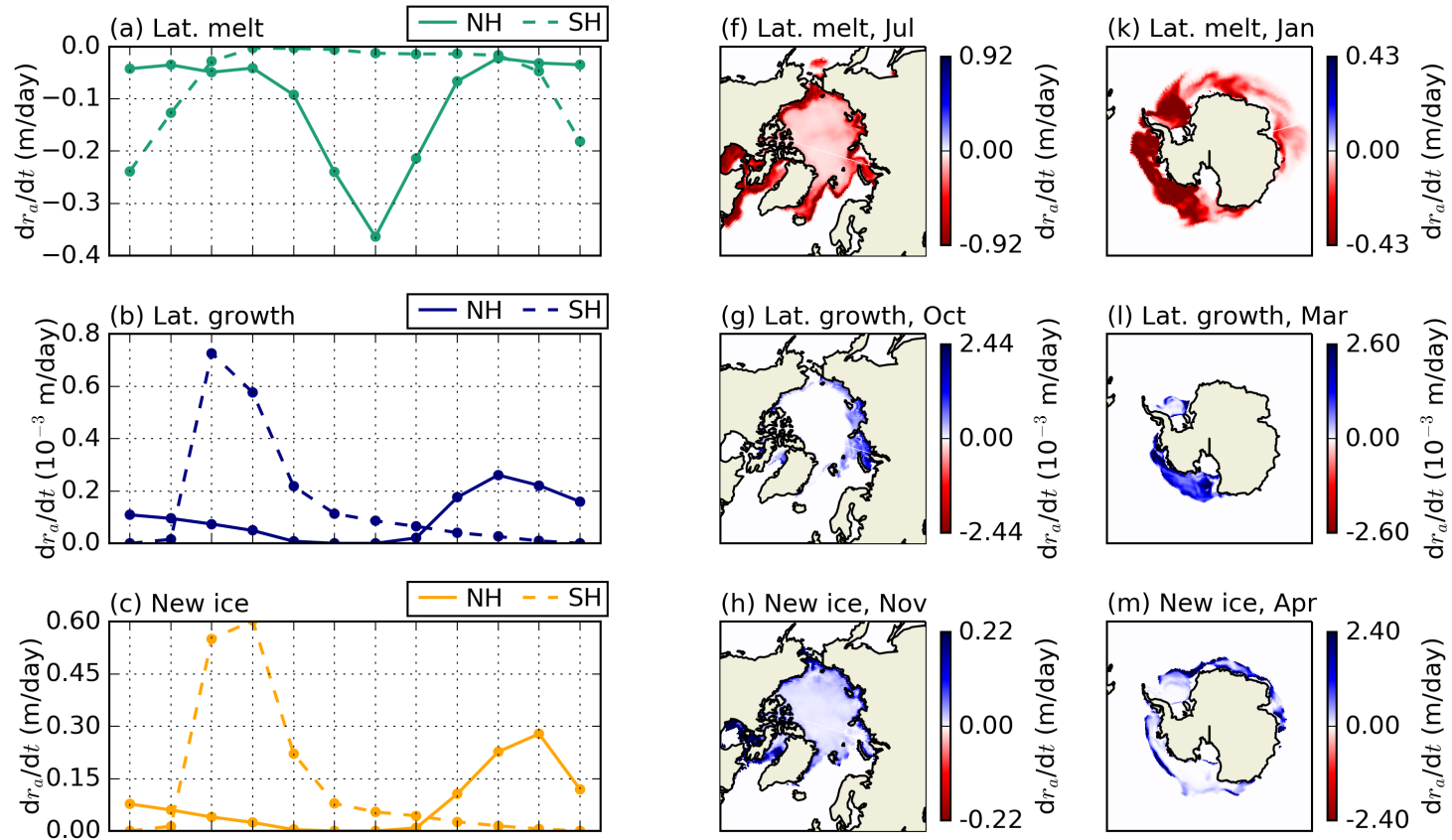

(m) New ice, Apr
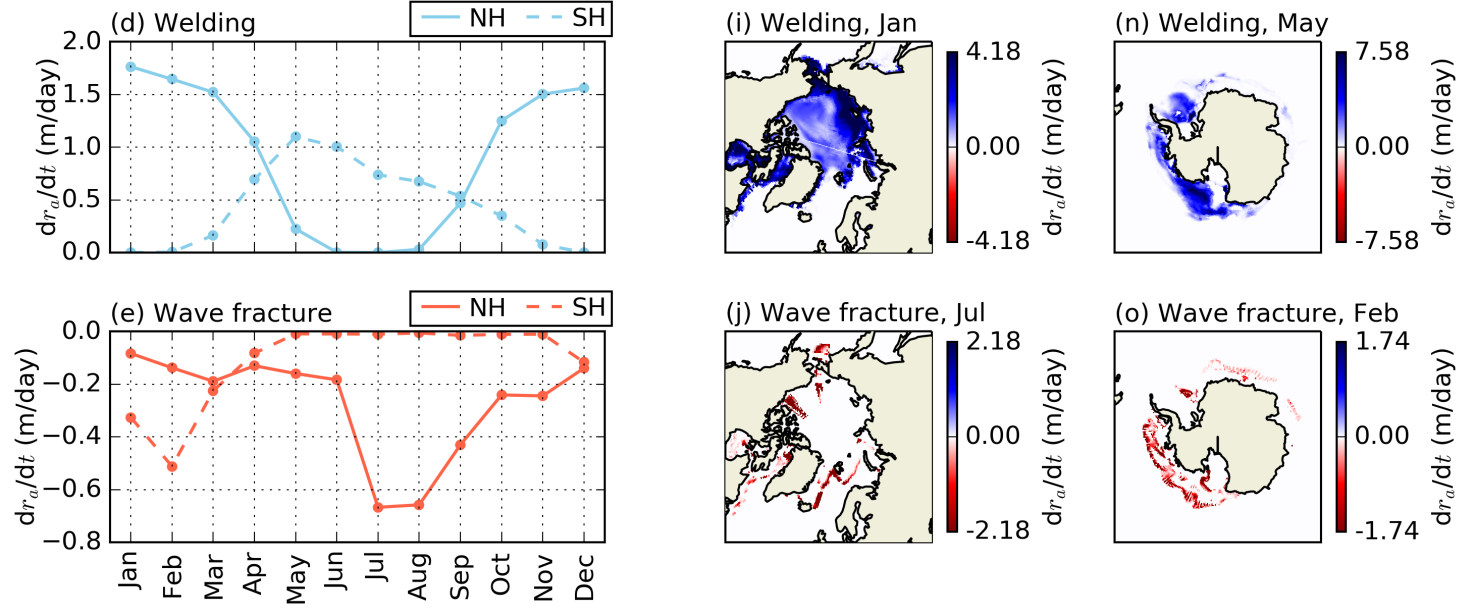

(o) Wave fracture, Feb

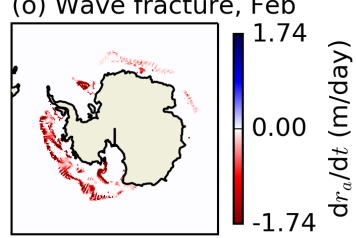

Figure 4.3: Seasonal and spatial variability of tendencies in representative radius, $r_{a}$. (a) The hemispheric average tendency in representative radius due to lateral melt for the $\mathrm{NH}$ (solid line) and SH (dashed line). (b-e) as (a) but for lateral growth, new ice production, floe welding, and wave fracture respectively. (f) Map of the tendency in $r_{a}$ due to lateral melt in the $\mathrm{NH}$ for the month with maximum average tendency, July, (see (a), solid line). (k) Map of the tendency in $r_{a}$ due to lateral melt in the SH for the month with maximum average tendency, January, (see (a), dashed line). (g-j) as (f) and (l-o) as (k) but for lateral growth, new ice production, floe welding, and wave fracture respectively. Note that lateral growth has units of $10^{-3} \mathrm{~m}$ day $^{-1}$, while other processes have units of $\mathrm{m} \mathrm{day}^{-1}$. 
(a) NH Mar

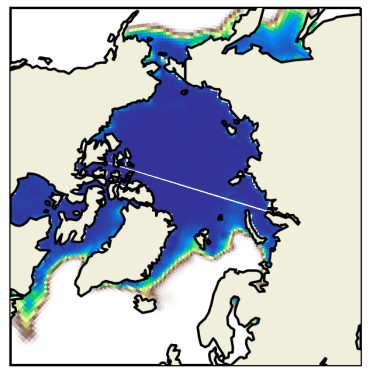

(b) NH Sep

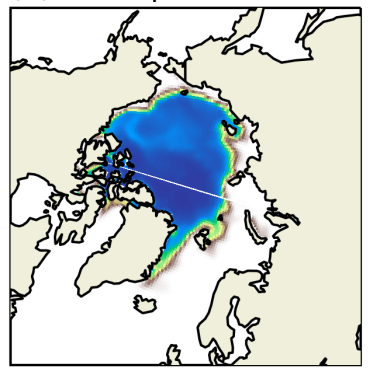

(c) $\mathrm{SH}$ Mar

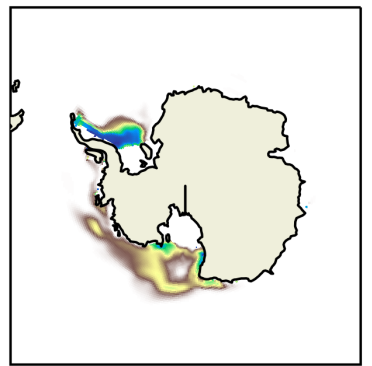

(d) SH Sep

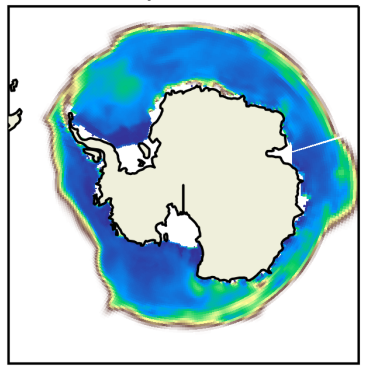

Sea ice concentration

$\begin{array}{llllll}0.0 & 0.2 & 0.4 & 0.6 & 0.8 & 1.0\end{array}$ (e) NH Mar

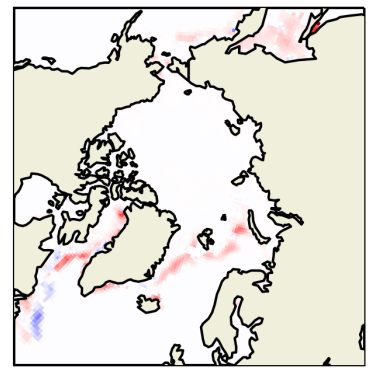

(f) NH Sep

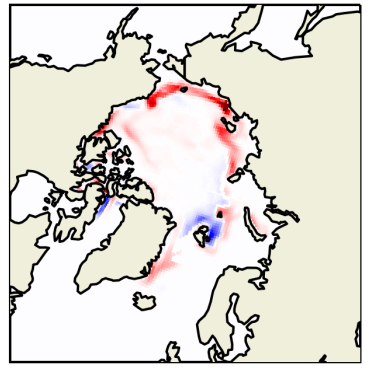

(g) $\mathrm{SH}$ Mar

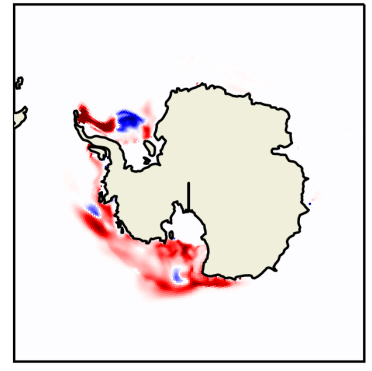

(h) SH Sep

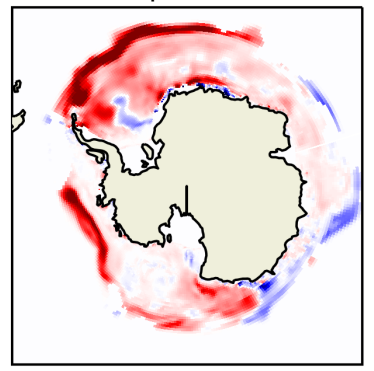

Difference

$\begin{array}{lllll}-0.2 & -0.1 & 0.0 & 0.1 & 0.2\end{array}$

Figure 4.4: Monthly sea ice concentration fields (out of maximum of 1) averaged over twenty years in the Northern and Southern Hemispheres for March (a, c, e, g) and September (b, d, f, h). The first column (a-d) shows the simulation from the standard model and the second column (b-h) shows shows the difference between the standard and FSTD models, where only differences that are significant at the $95 \%$ confidence level are shown. 
(a) $\mathrm{NH}$ Mar

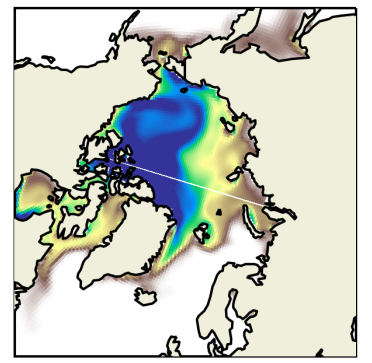

(b) NH Sep

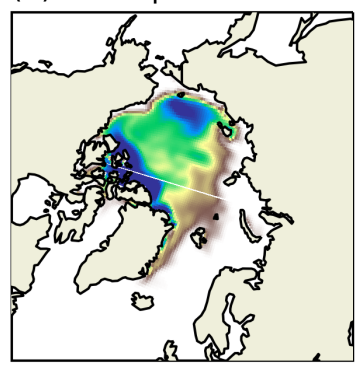

Sea ice thickness (m)

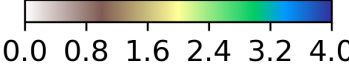

(c) $\mathrm{SH}$ Mar

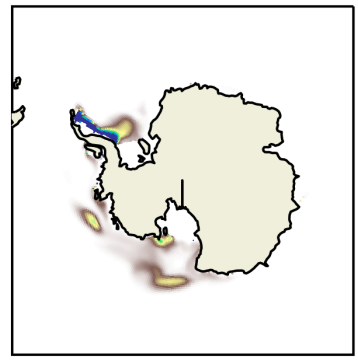

(d) SH Sep

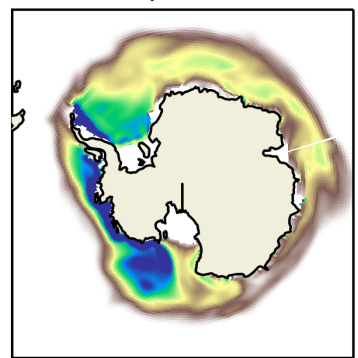

Sea ice thickness $(\mathrm{m})$

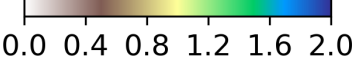

(e) $\mathrm{NH}$ Mar

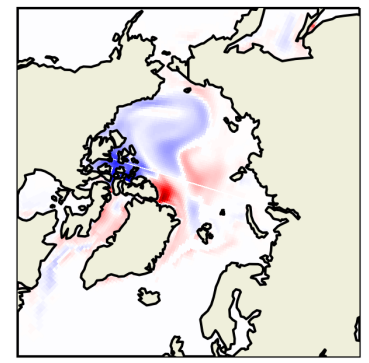

(f) NH Sep

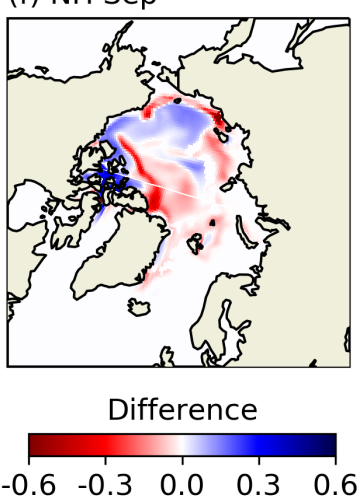

(g) SH Mar

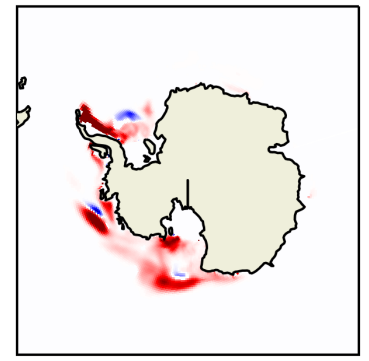

(h) SH Sep

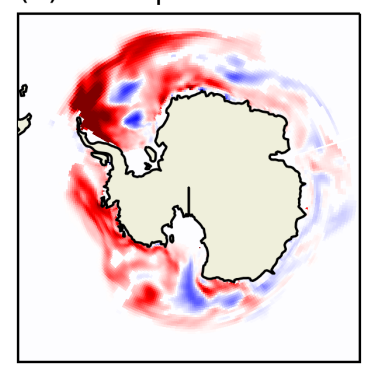

Difference

$\begin{array}{lllll}-0.3 & -0.15 & 0.0 & 0.15 & 0.3\end{array}$

Figure 4.5: As Fig. 4.4, but for sea ice volume per unit area (in m). 


\subsection{Discussion}

The results presented here demonstrate that the inclusion of floe size information has a significant impact on sea ice concentration and thickness, in agreement with Zhang et al. (2016) and Bennetts et al. (2017). The increase in lateral melt due to including a prognostic FSD reduces sea ice concentrations in both hemispheres, in an ocean-sea ice model with cyclic atmospheric forcing. This expanded model physics has the potential to alter sea ice feedbacks, climate sensitivity and the sea ice response to storms-impacts which will be investigated in future work.

The response of sea ice concentration and thickness to including floe size information in previous studies differ to those shown here, with both Zhang et al. (2016) and Bennetts et al. (2017) finding reductions but not increases in sea ice thickness, and Bennetts et al. (2017) finding larger reductions in sea ice concentration than the present study. Differences between model configurations and forcing scenarios in the various studies mean that we cannot directly compare the impacts on sea ice concentration and thickness results at this stage.

The sea ice model described here includes a more comprehensive description of physical processes that affect sea ice floe size than those included in other studies. The lack of observations of the FSD covering a region and time period large enough for global model validation means that we cannot discern which model simulates the most realistic FSD. This lack of observational data is precisely what motivates our fully prognostic approach, rather than constraining the FSD based on minimal data as in Zhang et al. (2016) and Bennetts et al. (2017). That we are able to capture some first-order characteristics of the FSD in our model experiments, which begin without initialization and allow the distribution to evolve freely, suggests that we have implemented some of the key physics that drive the FSD. These first-order characteristics include a varied spatial distribution of representative radius (Fig. 1), and a multi-scale number FSD (Fig. 2a-d) in line with observational studies (e.g. Steer et al. 2008).

Our process-based approach to model development allows us to examine the contri- 
bution of different processes to the FSD, with insights that are useful for future model development. Such results cannot be obtained from reduced complexity models which tune parametrizations to reproduce a certain FSD shape or behaviour. While introducing additional uncertain parameters, we hope that consideration of individual physical processes will motivate further study and help prioritize parameters that require further observational constraints. Model results could inform development of parametrizations used in simpler models in the future. Below, we discuss the contribution of different processes to the FSD and their representation in current models, as well as highlighting areas that require further work.

We find that the freezing together of floes is a key process in determining the evolution of floe size (Fig. 4.2, 4.3). In previous modelling studies, the choice of how to include floe merging or welding has been ad-hoc: Horvat and Tziperman (2015) do not discuss welding; Zhang et al. (2016) move all floes into the largest category if the ice growth rate exceeds a threshold determined by tuning model output to observations in the western Arctic; and Bennetts et al. (2017) double the floe diameter in a grid cell if the ocean freezing potential is positive. Floe welding has only recently been quantified in the field for the first time by Roach et al. (2018c), who found observational support for use of the geometric floe welding model described here, but additional observations are required to better constrain the floe welding parameter.

The fracture of ice by ocean waves is also important, with the redistribution of floes to certain preferred sizes (e.g. Fig. 4.2h) driving behaviour in the number FSD (e.g. Fig. $4.2 \mathrm{~d}$ ). We compute the new floe sizes generated by wave fracture explicitly, without requiring any assumptions about the FSD. In other parametrizations of wave fracture, Zhang et al. (2016) assume that wave-fractured ice is redistributed equally to all other categories of smaller size as a power law distribution. Their model depends strongly on a floe size redistribution 'participation factor', which they parametrize as a function of wind speed and open water fraction, fitting tuning constants in their model to cumulative number distributions observed in satellite images in the western Arctic. In Bennetts et al. (2017), floes fracture according to a strain criterion similar to ours, but the change in the FSD is calculated assuming a 'split power law' distribution of floes sizes based on observations from Toyota et al. (2011). Zhang et al. (2016) and Bennetts et al. (2017) impose behaviour on fractured floe sizes that is inconsistent with results from a small-scale model (Mon- 
tiel and Squire, 2017) and laboratory observations (Herman et al., 2018), which indicate preferred sizes in the FSD resulting from wave fracture. Developing or tuning models to explicitly match 'split power law' shapes may be misleading, as many observations do not show this distribution (e.g. Inoue 2004; Wang et al. 2016; Paget et al. 2001). Further, observations of a 'split power law' distribution could be interpreted as a gradual bending of curves rather than an abrupt transition (Herman, 2010).

In future work, the sea ice model should be coupled to a full spectrum ocean wave model with an appropriate treatment of wave energy damping by sea ice. There are certainly limitations with our attenuation scheme, which may not be suitable for small floes (Meylan, 2002) and neglects wave direction, unlike Bennetts et al. (2017). Sensitivity of the depth of wave penetration into the pack ice using different attenuation parametrizations such as Meylan et al. (2014) could be tested with our model, either using forcing data from a wave model hindcast or coupled to a wave model. Wave model coupling would also allow turbulent mixing due to ocean waves to occur within the sea ice region, influencing the heat fluxes available for sea ice melt and growth. More realistic simulation of waves in ice could also enable advances in the representation of sea ice growth (Roach et al., 2018c).

The choice of floe size assigned to new floes strongly impacts the simulated floe number distribution (Fig. 4.2). In our model, new ice is placed in the smallest floe size category, representing pancake ice formation. This results in large numbers of small floes during winter, a seasonality opposite to that obtained by Zhang et al. (2016). In reality, new frazil ice is herded into pancake floes only in the presence of surface waves and/or winds, while in the absence of wind and wave action frazil crystals freeze together to form large thin sheets of sea ice called nilas (Weeks and Ackley, 1986). Zhang et al. (2016) do not specify how they initialize floe sizes at the start of their simulation, nor the floe sizes at which new ice forms. Zhang et al. (2015) perform simple experiments that are initialized at the largest floe size. Bennetts et al. (2017) initialize their model using a constant floe diameter of $300 \mathrm{~m}$, and do not explain how the formation of new ice impacts the representative floe diameter. These models and the standard version of CICE could be considered to include nilas growth only. In contrast, our model includes pancake growth only, although the initial thickness of ice may correspond better to nilas growth than pancakes. Future models should ideally incorporate both nilas and pancake growth, 
perhaps using some critical value of the tensile stress mode arising from the wave field (Shen, 2004) to determine which growth type occurs.

Of the five processes that determine the FSD, only new ice formation and lateral melt and growth cause changes to sea ice concentration in our model. We find that lateral growth, which was not included in either Zhang et al. (2016) or Bennetts et al. (2017), is around two orders of magnitude smaller overall than lateral melt (Fig. 4.2e-h). Roach et al. (2018c) find that the lateral growth model used here underestimates growth rates of small ice floes observed in the Arctic Ocean during fall. More observations are required to determine whether the model underestimates lateral growth rates in other conditions.

Here, lateral melting is a significant process for evolution of the sea ice FSD (Fig. 4.2e-h) and is a function of the FSD itself. In contrast, the lateral melt formulation in Zhang et al. (2016) assumes all floe size categories have the same ITD, and does not parametrize the effect of lateral melting on the FSD (the second term in Eqn. 4.14). Bennetts et al. (2017) use a single representative floe size in each grid cell, neglecting the sub-grid-scale distribution of floe sizes, which could vary over a broad range. All three models demonstrate that lateral melt has large impact on simulated sea ice concentration, also motivating further observational validation. As noted by Roach et al. (2018a), the parametrization of lateral melt rate used in our model and standard CICE5.1, as well as other models, is based on a single field study of a single floe (Maykut and Perovich, 1987). Further constraints on individual processes like this, which strongly impact the sea ice FSD, could greatly assist model development, particularly in the absence of global observations of floe sizes.

\subsection{Conclusions}

In this study, we have presented a scheme for modelling a fully prognostic joint sea ice floe size and thickness distribution. We have examined model results in both hemispheres obtained without initialization or tuning parameters to obtain a particular floe size distribution, unlike previous studies. We find that the five processes implemented here-lateral melt and growth of floes, floe welding in freezing conditions, new ice formation and fracture of floes by ocean surface waves-capture some first-order characteristics of the floe 
size distribution.

However, definite statements on the realism of the simulated distribution are hindered by a lack of global observations of floe size distribution. Observations which cover a large spatial and temporal region at small enough resolution are not yet available. This lack of observations is the motivation for constructing a model which does not assume $a$ priori distributions for simulated floe sizes. This general framework makes any additions or modifications to physical processes straightforward to implement. Future additions may include dynamics more appropriate for the marginal ice zone (e.g. Rynders et al. 2016), floe-size-dependent mechanical redistribution (e.g. Horvat and Tziperman 2015), dependence of form drag on the simulated floe size distribution, two clearly defined sea ice growth pathways (nilas and pancake growth), and coupling with an ocean wave model.

In spite of our choices to keep much of the physics consistent with the standard model, impacts on sea ice concentration and thickness caused by the addition of a floe size distribution are significant. This suggests that small scale processes associated with individual floes may be important for the polar climate system. The observed predominance of sea ice growth via pancake formation in the Antarctic (Wadhams et al., 1987) suggests that these processes may be particularly relevant for the Southern Hemisphere. Moreover, the predicted increase in the Arctic marginal ice zone (Aksenov et al., 2017) implies that processes at the sea ice floe scale may become more important for simulation of sea ice in the future. The model presented here could help to answer questions on the seasonal evolution of floe size in the polar oceans, the possibility of power law emergence from interactions at the floe scale in a climate model, and the degree to which sea ice melting is influenced by fractured sea ice cover. 


\section{Article citation}

Roach, L. A., C. Horvat, S. M. Dean, and C. M. Bitz (2018b), An emergent sea ice floe size distribution in a global coupled ocean-sea ice model, Journal of Geophysical Research: Oceans, 123(6), 4322-4337, doi:10.1029/2017JC013692

\section{Acknowledgements}

L. Roach and S. Dean were funded via Marsden contract VUW-1408. C. Horvat was supported by the Frank Knox Memorial Fellowship during parts of this work. C. Bitz was supported by the US National Science Foundation PLR-1643431. The authors would like to thank Richard Gorman for producing the wave model hindcast, Erik Behrens for setting up the standard model NEMO-CICE configuration, and Elizabeth Hunke and an anonymous reviewer for their consideration of the manuscript. The authors also wish to acknowledge the contribution of NeSI high-performance computing facilities to the results of this research. NZ's national facilities are provided by the NZ eScience Infrastructure and funded jointly by NeSI's collaborator institutions and through the Ministry of Business, Innovation \& Employment's Research Infrastructure programme. Model results are publicly available via Zenodo, (https: / / doi.org/10.5281/zenodo.1193929). Access to the code via a Github repository is available from the corresponding author upon request. 


\section{Chapter 5}

\section{Quantifying growth of pancake sea ice floes using images from drifting buoys}

Development of the sea ice floe size model in the previous chapter highlighted the lack of observational studies that could be used to constrain descriptions of floe freezing processes. This motivated the present chapter, published as Roach et al. (2018c), which was conducted in parallel to the previous chapter. Here, we analyse images captured by nearLagrangian drifting wave buoys to obtain floe size evolution. Our analysis represents the first in-situ quantification of lateral floe growth and floe welding. Comparing observational results to model process descriptions provides useful insights for both Chapter 4 and Chapter 6.

\subsection{Abstract}

New sea ice in the polar regions often begins as small pancake floes in autumn and winter that grow laterally and weld together into larger floes. However, conditions in polar oceans during freeze-up are harsh, rendering in-situ observations of small-scale sea ice growth processes difficult and infrequent. Here, we apply image processing techniques to images obtained by drifting wave buoys (SWIFTs) deployed in the autumn Arctic Ocean to quantify these processes in situ for the first time. Small pancake ice floes were observed to form and grow gradually in freezing, low-wave conditions. We find that pancake floe diameters are limited by the wave field, such that floe diameter is proportional to wavelength and amplitude over time. Floe welding correlates well with sea ice concentration, and the observations can be used to estimate a key model parameter for floe size evolu- 
tion. There is some agreement between observed lateral growth rates and those predicted using a theoretical model based on heat flux balance, but the model lateral growth rates are too conservative in these conditions. These results will be used to inform description of lateral floe growth and floe welding in new models that evolve the sea ice floe size distribution.

\subsection{Introduction}

Formation of sea ice in the polar regions is strongly coupled to the ocean and atmosphere. When the upper ocean layer is at or slightly below the freezing point, small frazil crystals begin to form. Sheets of sea ice can form under two different pathways, depending on the wind and wave conditions. In quiescent conditions, frazil crystals freeze together to form nilas, thin sheet ice that is initially transparent. Nilas then grows in thickness by congelation growth on the underside of the ice. In more dynamic conditions with wind and wave forcing, semi-consolidated frazil slush can oscillate and form near-circular floes with upturned edges called pancakes (Weeks and Ackley, 1986). These may grow laterally, by further adfreezing of frazil crystals (Wadhams et al., 1987). When pancakes remain in contact for sufficient periods of time, they may freeze together (Shen and Ackley, 1991). The final step in this sequence is the transition from loosely joined pancakes to a complete sheet of joined, or cemented, pancakes (Weeks and Ackley, 1986). Sea ice resulting from pancake growth is likely to be rougher than that resulting from nilas growth, thus impacting oceanic and atmospheric fluxes. Pancake sea ice formation is common in the Antarctic (Wadhams et al., 1987) and is becoming more common in the increasingly ice-free Arctic (Jones, 2009) where new ice growth is subject to more active wave fields (Thomson and Rogers, 2014).

However, large-scale sea ice models, such as those used in global climate models, typically describe only sea ice growth by formation of nilas. For example, the state-of-the-art sea ice models CICE5 and LIM3 form some new volume of sea ice from flux balance and preferentially set the new ice to have a low thickness covering a large surface area (Hunke et al., 2015; Vancoppenolle et al., 2009). Like CICE5 and LIM3, most sea ice components of global climate models employ a probability distribution in sea ice thickness in order to calculate thermodynamic and dynamic sea ice evolution (Hunke et al., 2010). They 
do not include information on sea ice floe size, which means they are currently unable to represent sea ice growth by formation of pancakes.

Recent work by Horvat and Tziperman (2015), Horvat and Tziperman (2017), Zhang et al. (2015), Bennetts et al. (2017) and Zhang et al. (2016) has, however, laid the foundations for the inclusion of a sea ice floe size distribution, either defined in parallel or coupled to the ice thickness distribution, in current sea ice models. Including a floe size distribution would allow a fuller description of physical processes such as lateral melt, dynamic floe collisions and floe fracture, as well as lateral growth. These processes are either not currently included in models (lateral growth and floe fracture) or heavily parametrized (lateral melt and floe collisions - see e.g. Hunke et al. 2015). Several models (e.g. CICE, Hunke et al. 2015) include parametrizations for lateral melt based on observational studies. For example, a functional form for a lateral melt rate was found in laboratory work by Josberger and Martin (1981), which was fit to data obtained in the Arctic by Maykut and Perovich (1987), and is used in models today.

Fewer empirical results exist for sea ice growth processes than for melt processes, largely due to the inaccessibility of polar regions during winter. Recent modelling studies show that observational constraints on sea ice freezing processes are required. In the context of simulated floe size distributions, Horvat and Tziperman (2015) presented a scheme for lateral growth of sea ice floes, where the growth rate was computed by a geometrical partitioning of the ocean freezing potential. They do not cite any observational justification for this.

Neither Bennetts et al. (2017) nor Zhang et al. (2016) include lateral growth, but they both identify a separate growth process referred to as floe bonding (Bennetts et al., 2017) or floe welding (Zhang et al., 2016). This is the transition from loosely joined pancakes to cemented pancakes mentioned above, which is a freezing-together of floes, rather than a dynamic process, impacting only the floe size distribution and not sea ice concentration. Zhang et al. (2016) notes that the coalescence of sea ice floes is 'difficult to determine' because of 'lack of knowledge about the welding processes.' As there are no empirical observations justifying the appropriate rate of welding, Bennetts et al. (2017) represent welding processes by doubling their representative floe diameter (up to a maximum diameter) each time step in grid cells where the ocean temperature is below freezing. Similarly, 
Zhang et al. (2016) move all floes into the largest floe size category when the ice growth rate in a certain grid cell exceeds a certain threshold, a number which is chosen as part of their model tuning.

Roach et al. (2018b) include both lateral growth and floe welding. The welding scheme proposed uses the geometric probability of sea ice floes touching during freezing conditions, where the coefficient of proportionality is the rate of welding participation. A key aim of the present study is to constrain this welding rate constant.

While some field studies exist on the transition from frazil to pancake ice (Doble, 2009) and the kinematics of a pancake ice field (Doble and Wadhams, 2006; Rottier, 1992), to our knowledge there are no published field measurements of lateral floe growth or rates of floe welding. Using a one-dimensional model Shen and Ackley (1991), showed that wave action can initiate and maintain collisions between floes on the order of seconds and suggest that floes can freeze together if this timescale is long enough, as observed in the field. We do not know of any laboratory observations of such floe welding interactions. Lateral growth of pancake sea ice has been measured in laboratory wave-ice flumes (Shen and Ackley, 1995; Leonard et al., 1998; Onstott et al., 1998; Shen, 2004), providing estimates of pancake formation on the time scale of hours to days. Shen (2004) theorized that the ultimate diameter of pancake ice will be limited by tensile stress induced by the wave field. They found good correlation between observed maximum floe diameter and the observed tensile stress mode, with additional dependence on temperature and salinity, in a cold water tank. Results from laboratory experiments may not hold under different environmental conditions (Shen and Ackley, 1995) and do not always scale correctly to the real world (Doble, 2009), requiring validation by field observations.

The aim of the present study is to quantify the rate at which pancake sea ice floes grow laterally and weld together, processes that have been identified as important for sea ice model development. Pancake sea ice growth processes are observed in situ using time lapse images of the ocean surface in the autumn Arctic Ocean. A supervised image processing algorithm, described in Sec. 5.4, is used to determine floe sizes and welding events. We describe the evolution of floe size and environmental conditions and quantify errors associated with image processing in Sec. 5.5. In Sec. 5.6-5.8 we compare observations to predictions by three theoretical models, which describe different processes 
that would all be required to fully model evolution of sea ice floe size during freezing conditions. The three models describe the limiting diameter of pancake ice arising from stress induced by the wave field (Shen, 2004), evolution of the floe size distribution due to welding (Roach et al., 2018b) and evolution of the floe size distribution due to lateral growth (Horvat and Tziperman, 2015). The first of these has been validated by laboratory experiments. The latter two are used to evolve the floe size distribution in models and have not been constrained empirically. The observational analysis presented here evaluates these models to determine how atmospheric conditions and waves affect growth of pancake ice. These results are useful for future sea ice model development.

\subsection{Data}

The images used in this study were captured by SWIFT buoys (Surface Wave Instrument Floats with Tracking), which are free drifting systems designed to measure ocean waves, winds and near-surface turbulence (Thomson, 2012). SWIFTs are also equipped with serial cameras (4D systems uCam) mounted on a mast $1 \mathrm{~m}$ above the water surface, which capture images of their surroundings in five bursts each hour. Raw (un-orthorectified) images have a resolution of $320 \times 240$ pixels. The bursts are approximately eight minutes long, recording 102 images at a frequency of $0.25 \mathrm{~Hz}$, with the next four minutes allocated to processing data. An example image is shown in Fig. 5.1a.

Wave spectra are determined from measurements of orbital velocity components (Herbers et al., 2012), made using a Microstrain 3DM-GX3-35 combination GPS receiver and Inertial Motion Unit (IMU), at a frequency of $4 \mathrm{~Hz}$. GPS horizontal velocities have a resolution of $5 \mathrm{~cm} / \mathrm{s}$, which is sufficient to resolve most wave orbital motions (Thomson, 2012). Bulk wave parameters are calculated from the wave spectra following the standard formulations as defined in Herbers et al. (2012). Significant wave height, $H_{s}$, is calculated as $H_{s}=4 \sqrt{m_{0}}$, where $m_{0}$ is the first moment of the spectrum. The energy-weighted period, $T_{e}$, is $T_{e}=\frac{m_{0}}{\sum E f}$, where $E$ and $f$ are the energy and frequency respectively. Typical errors associated with $H_{s}$ and $T_{e}$ estimates are less than a few percent (Herbers et al., 2012). Water temperature and salinity $0.5 \mathrm{~m}$ below the surface are measured with an AADI Aanderaa Conductivity Sensor 4319. Air temperature is measured using an ultrasonic anemometer (Airmar PB200) $1 \mathrm{~m}$ above the surface, with a resolution of $0.1{ }^{\circ} \mathrm{C}$. 
(a)

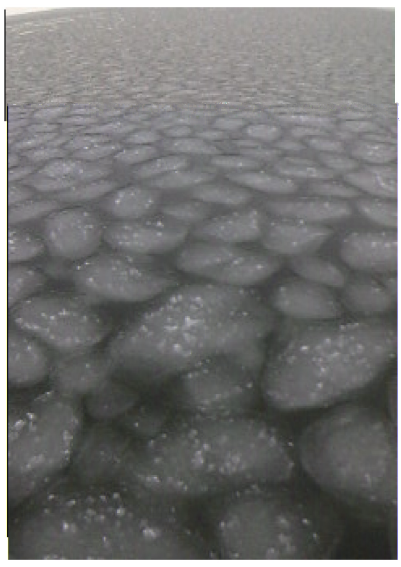

(c)

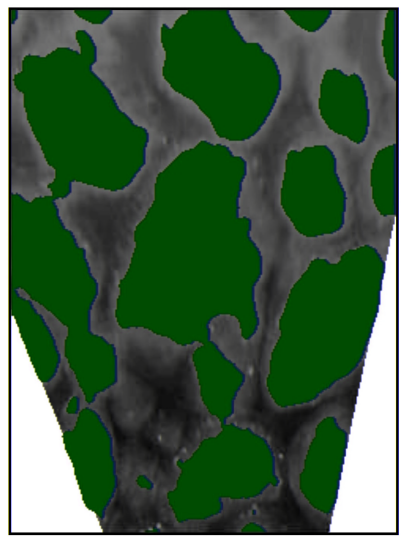

(b)

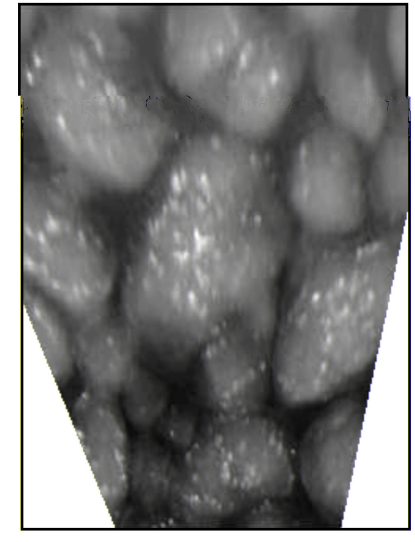

(d)

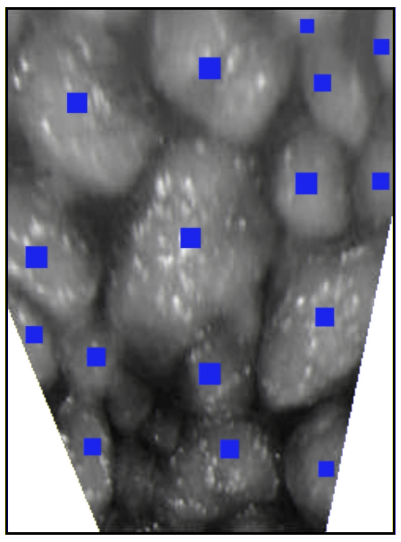

(e)

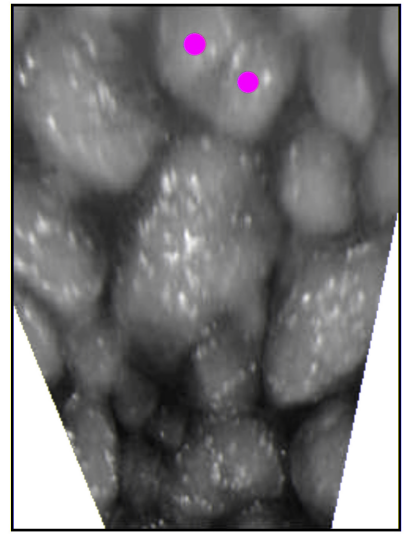

Figure 5.1: An example SWIFT image (a) as captured, (b) orthorectified, (c) with the area estimate from a fixed threshold (blue shading), (d) with the discrete floes found by a user-specified threshold (red squares), and (e) with the floe components found by a user-specified threshold, which split one discrete floe from (d) into two floe components (yellow circles).

More details on the platform can be found in Thomson (2012).

Two SWIFTs were deployed at approximately $72^{\circ} 29^{\prime} \mathrm{N} 158^{\circ} 41^{\prime} \mathrm{W}$ from the $R / V$ Sikuliaq October 26-27, 2015, during the Sea State cruise in the Beaufort Sea region of the Arctic Ocean. Map of SWIFT buoy deployment and recovery locations relative to sea ice area and land are shown in Fig. 5.2. More details on the cruise and the context of these measurements can be found in Thomson et al. (2018). The buoys were left to drift for two days under freezing conditions and relatively low sea state. The buoys remained within $1 \mathrm{~km}$ of each other over the course of the deployment pe- 

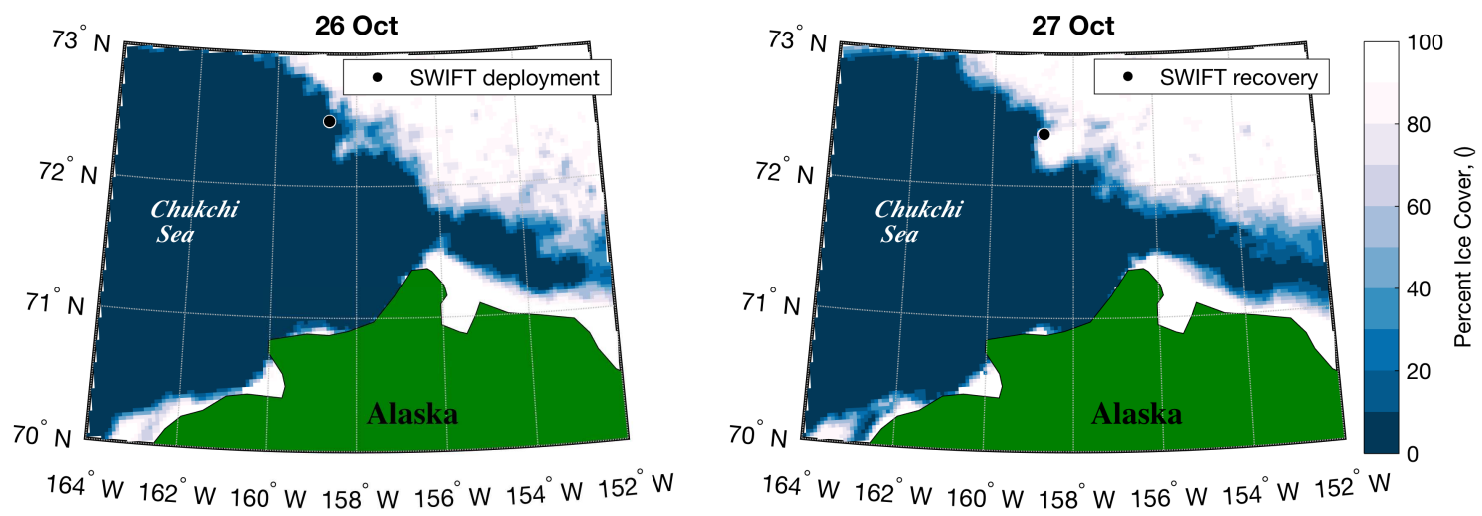

Figure 5.2: Map showing sea ice coverage in area of SWIFT observations. SWIFT deployment and recovery locations (black points) are shown in context of ice concentration and extent from daily AMSR2 sea ice product (Spreen et al., 2008) from October 26 (left) and October 27 (right). Note that SWIFT 09 and 14 deployment and recovery locations are within $1 \mathrm{~km}$, such that both can be represented by one point.

riod. As confirmed by observers onboard the ship, the buoys were initially surrounded by open water, that froze, forming small pancake floes whose size increased over time. The evolution of the floe sizes in time can be seen in the videos provided as online supplementary material (Supporting Information for Roach et al. 2018c, available at http://doi.wiley.com/10.1002/2017JC013693).

While buoys were drifting, shipboard measurements were being made in the surrounding area. Of primary interest to this study are atmospheric flux measurements, estimated from a suite of instruments installed on the ship's mast, estimates of water temperature made from a towed thermistor ('Sea Snake'), and sea ice thicknesses made from hourly dip net samples.

Net atmospheric fluxes, $Q_{n e t}$, were determined using shipboard turbulent flux measurements as

$$
Q_{n e t}=Q_{s i}-Q_{s o}+Q_{l i}-Q_{l o}-L H F-S H F
$$

$Q_{s i}$ is incoming (downwelling) shortwave radiation, $Q_{s o}$ is outgoing (upwelling) shortwave radiation, $Q_{l i}$ is incoming (downwelling) longwave radiation, $Q_{l o}$ is outgoing (upwelling) longwave radiation, $L H F$ is latent heat flux, and $S H F$ is sensible heat flux. Details of the specific measurement methods and calculations can be found in Persson (2012) and Persson (2002). The estimate of net atmospheric flux $\left(Q_{n e t}\right)$ has an accuracy of $10 \mathrm{~W} \mathrm{~m}^{-2}$. 
The dip net is used to retrieve between 3 and 6 individual pancake ice samples over the side of the ship which are then manually measured (Wadhams et al., 2018). Ice thickness estimates are determined as an average of the observed thicknesses.

\subsection{Methods}

Images captured by the SWIFTs are processed using a series of standard image processing techniques in order to calculate (i) a rate of floe welding events and (ii) a lateral floe growth rate. The key methodological approach that allows us to separate these two concurrent processes is use of three levels of image brightness thresholding. Firstly, images are processed to obtain estimates of sea ice area; secondly, to obtain estimates of the number of discrete sea ice floes; thirdly, to obtain estimates of the number of floe 'components' that, together, make up welded floes. In this latter step, we search within each discrete floe to see if brightness thresholding will further isolate any floe components within composite floes.

Quantifying growth of sea ice floes from these images requires that the drift of SWIFT buoys is Lagrangian with respect to the drift of sea ice. Lund et al. (2018) show that SWIFTs drift with the surrounding ice, so we can assume that we are observing the same ice through time in a statistical sense, even though the sequence of images does not capture the exact same floes due to the random motion of the floes in and out of the camera frame. We further assume that each burst is representative of the ice conditions in that 12 minute window. We sample ten images within each burst, selected at random, and then average each set of ten calculated ice properties in time. Naturally, since the images were taken in dynamic conditions, floes will touch and move apart. The choice of time averaging was designed to average out these temporary floe interactions.

Each selected image is undistorted based on intrinsic camera parameters using the undistortImage function in Matlab's image processing toolbox, and orthorectified using the imwarp function, where the transformation object is determined by fitting a geometric transform to calibration images. This results in a bird's eye view of the image in real-world coordinates (Fig. 5.1b), where 1 pixel in the image corresponds to $4.6 \mathrm{~mm}$ in real-world coordinates. 
Orthorectification assumes pitch and roll are zero such that the ocean surface is always oriented the same relative to the camera. Although pitch and roll values are recorded by the IMU in the SWIFT buoys, the IMU is not synchronized with image acquisition and thus cannot be used for orthorectification. Instead, average pitch and roll values recorded by the SWIFTs for each data burst are used to calculate the associated error in area that would result at the far edge of the orthorectified image, where the largest error due to buoy motion occurs.

Due to the small scale of the images, the differences in pixel intensity over ice and water are fairly uniform - apart from a front-to-back luminosity gradient-meaning that only simple image processing techniques are required. Colour information is removed and the linear front-to-back luminosity gradient is corrected. We then apply simple image processing functions from the Open Source Computer Vision Library (OpenCV, Itseez 2015). A bilateral filter removes noise from the image, while keeping the edges sharp, by applying Gaussian filters as function of space and as a function of pixel intensity. Next, fixed-level brightness thresholds are applied to binarize the image. We trialled the watershed algorithm, a geometric approach commonly used in floe size studies (e.g. Arntsen et al. 2015), but we found that this erroneously separated the rather long, oblong-shaped floes that appeared in some images. Therefore only brightness thresholding is used to analyse the images here.

Visually, a single fixed threshold (97) gave a good separation of solid ice and water, from which the total ice area, $\mathcal{A}$, and concentration, $C$, were calculated (Fig. 5.1c). This thresholding excludes the loose frazil crystals on floe edges. Frazil aggregations-which are very young pancakes where crystals have begun to freeze together but may not yet be completely consolidated-are included as solid ice. Other properties were calculated from each image by varying the fixed threshold to a value determined by eye. After thresholding, the OpenCV function for identifying contours in a binary image, retrieving only the extreme outer contours, can be used to count floes. Besides the total ice area, the key properties required for the analysis are the number of discrete floes, $N_{d}$, and the number of floe components, $N_{c}$, which includes all components of composite floes as well as non-composite floes. If there are no welded or composite floes then $N_{c}=N_{d}$. If there is at least one welded or composite floe, $N_{c}>N_{d}$. 
First, a fixed threshold is found that gives an accurate number of discrete floes, $N_{d}$ (Fig. 5.1d). Then a second fixed threshold is applied within each contour identified in the first step to calculate the number of floe components, $N_{c}$, within discrete floes (Fig. 5.1e). The user thus decides whether floes are temporarily touching or welded together. As we expected there to be some subjectivity to this process, the algorithm was run twice by two different users. Comparing results from both trials gives some estimate of the error arising from the human component of the image analysis.

The initiation of each step is automated so that the user can run the code, produce figures and adjust sliders to select thresholds where floe welding is included or ignored. If the user cannot find a threshold which returns an accurate number of floes, the user discards the image and it is replaced with another in that burst. In some bursts, it is not possible to find thresholds which give realistic numbers of floes; these bursts are ignored.

We define a representative floe area, for both the number of discrete floes $\left(N_{d}\right)$ and the number of all floe components $\left(N_{c}\right), \bar{A}_{d}\left(\bar{A}_{c}\right)$ by assuming that all floes in an image have the same size, so $\bar{A}_{d}=\mathcal{A} / N_{d}\left(\bar{A}_{c}=\mathcal{A} / N_{c}\right)$. We choose this representative floe area as a metric because there is not much variation of floe size within each image: on average $80 \%$ of floe areas are within one order of magnitude of the mean. Equivalent radii, $r$, or diameters, $2 r$, are calculated using $A=4 \cdot 0.66 r^{2}$ (Rothrock and Thorndike, 1984), which accounts for non-circularity of floes.

Floe welding will increase the representative floe area because the total number of floes decreases, without changing the total ice area. Lateral floe growth will increase the total ice area as individual floes increase in area, without changing the total number of floes. Thus, the time rate of change of the representative area of discrete floes, $\bar{A}_{d}$, describes the evolution of floe size under both floe welding and lateral growth, while the time rate of change of the representative area of floe components, $\bar{A}_{c}$, describes evolution of floe size under lateral growth only. The separation of these two processes in the analysis requires conservation of ice area during floe welding. It is possible that the welding of two floes traps and consolidates loose frazil crystals in between them, thus violating conservation of total ice area, but we are unable to confirm this without more advanced image processing methods. 
We include floes which are cut off by the image boundary, although this may cause a low bias in the average floe area. Excluding them would greatly reduce our sample size. Accuracy in absolute area values is not of primary importance as our analysis principally concerns growth rates or relative area values. When varying thresholds to identify contours in the image, it was noted that the algorithm may erroneously identify small specks as discrete objects. These appear to be points of high reflection from the ocean or crystals caused by orientation of the surface. To eliminate this effect, any individual floe areas which are less than $3 \%$ of the representative floe area are discarded in post-processing. This step impacts the floe counts $\left(N_{c}\right.$ and $\left.N_{d}\right)$ and individual floe areas.

The areas of individual discrete floes and floe components within each image are also calculated, but the algorithm performed more poorly here and so these are only used once in our analysis (to calculate the floe size distribution to compare to a theoretical lateral growth model in Sec. 5.8). The individual areas are calculated from the thresholds used to count discrete floes and floe components (e.g. Fig. 5.1d and e respectively). To account for the lower accuracy of floe area estimates, individual areas are subsequently corrected by a normalization to the total ice area estimate obtained from the initial threshold (e.g. Fig. 5.1c), $\mathcal{A}$, i.e.

$$
A_{c}^{n}=A_{c}^{n} \frac{\mathcal{A}}{\sum_{n} A_{c}^{n}}
$$

where $n$ denotes individual areas. Thus underestimated individual floe areas are increased in proportion to their size such that they sum to the total ice area $\mathcal{A}$.

We proceed by describing how floes evolve in the study time period with environmental conditions, and then compare observational results to three models which describe floe size in Sec. 5.6-5.8 below.

\subsection{Floe size evolution}

Fig. 5.3 shows the representative area of discrete floes and the representative area of floe components over the deployment period. The range of values observed is similar for both SWIFTs, and both show generally increasing areas, with some variability. The time variability, between results from the ten images from each 12 minute burst (shaded region in 


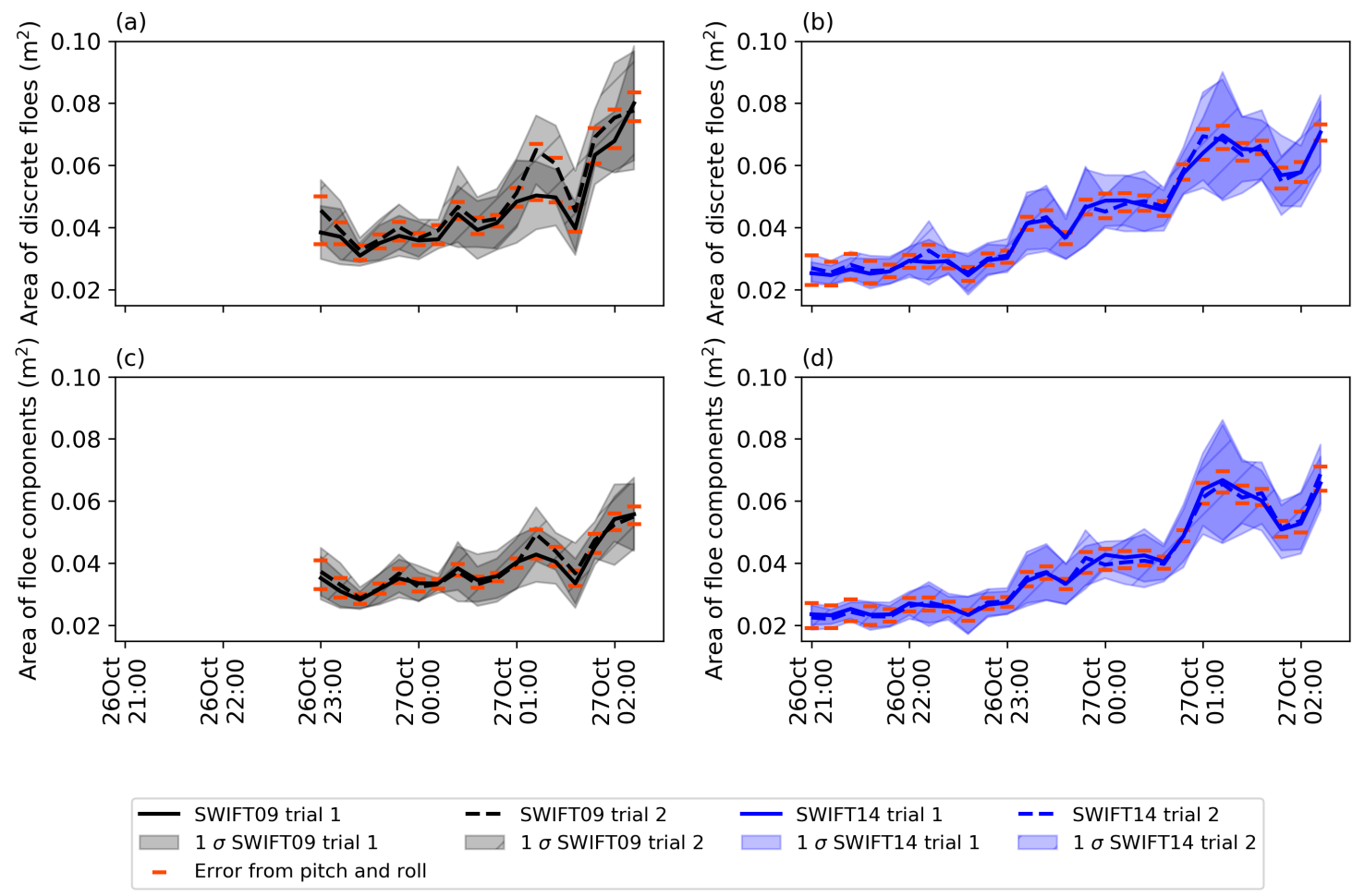

Figure 5.3: Burst-averaged floe areas over the deployment period where (a-b) show the representative area of discrete floes, and (c-d) shows the representative area of floe components. Solid and dashed lines represent algorithm results from two different users. Shaded areas indicate \pm one standard deviation in time for each burst, with the solid shaded region corresponding to the solid line, the dashed shaded region corresponding to the dashed line, and the darker shaded region corresponding to the overlap between the two standard deviation envelopes. Error bars (orange) show the error arising from assuming that pitch and roll are zero.

Fig. 5.3) is non-negligible, but does not obscure the overall behaviour. The time variability is likely to be mostly due to floe motion; in the sequence of images, floes are observed to move in and out of the frame (Supporting Information for Roach et al. 2018c, available at http://doi.wiley.com/10.1002/2017JC013693).

We find that time variability is larger than error in area estimates arising from image processing, due to both subjectivity and wave motion. The two trials by different users show strong agreement (Fig. 5.3), with a Pearson correlation coefficient of $99 \%$ for identification of discrete floes and $96 \%$ for identification of floe components. Time variability (shaded regions in Fig. 5.3) exceeds the difference between the two user trials for all image bursts except one from SWIFT09 (which occurs at approximately 01:12). This strengthens confidence that the human component in estimates of floe areas and lateral growth rates is negligible, and we will henceforth show only the midpoint of the two 
trials. Errors due to pitch and roll range from upwards of $20 \%$ at the beginning of the period, and around $2 \%$ towards the end of the period, and are shown as error bars in Fig. 5.3. We find that the error due to pitch and roll is relatively small, and smaller than the sub-burst time variability except at the very start of the SWIFT14 deployment.

Focusing on overall behaviour, by using only the average values from the ten images sampled in each 12 minute window, Fig. 5.4 shows evolution of representative floe area. The grey shading shows the contribution of lateral floe growth, computed from the representative area of floe components. The residual growth is the impact of floe welding on the representative floe area, which is much smaller than lateral growth.

Overall, we see a steady lateral growth throughout the deployment, of larger magnitude in SWIFT14 than SWIFT09. Welding area growth estimates are approximately constant in time from SWIFT14, but accelerate towards the end of the deployment period for SWIFT09. These differences between the ice evolution observed by the two SWIFTs may arise from slightly different environmental conditions. Generally the two SWIFTs observe similar floe size evolution, as we would expect based on their proximity. The representative areas from the two SWIFTs correlate well over the period where results are available from both, with a Pearson correlation coefficient of 0.66 for discrete floes and 0.70 for floe components (both $p<1 \%$ ).
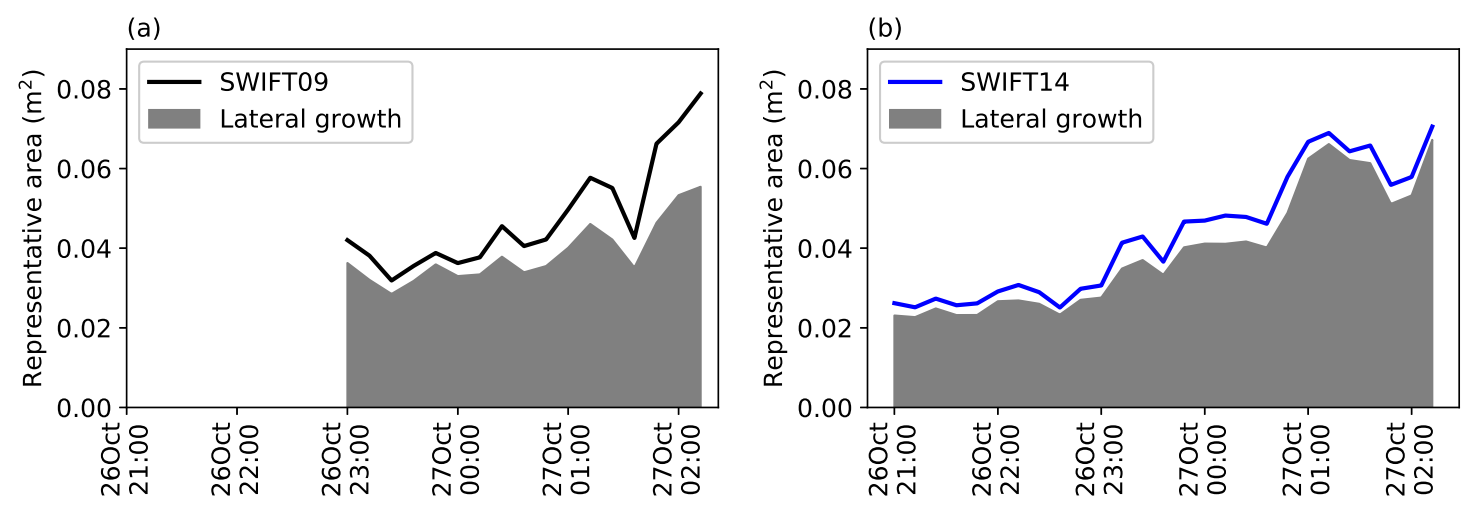

Figure 5.4: Evolution of burst-averaged representative areas of discrete floes, where the grey shading shows the contribution of lateral growth for (a) SWIFT09 and (b) SWIFT14. 
Atmospheric and ocean variables measured continuously from the ship, including net atmospheric heat flux, sea surface temperature, and ice thickness, are not measured by SWIFT buoys and provide context for observations of ice growth. As the ship was often at long distance away from the buoys during the deployment period, we only use measurements from the ship when within $10 \mathrm{~km}$ of the buoy locations (Fig. 5.5). The net atmospheric flux measured from the ship over the period was strongly negative, from the ocean to the atmosphere, ranging between -200 and $-170 \mathrm{~W} \mathrm{~m}^{-2}$. Sea surface temperatures measured from the ship dropped over the first few hours of ice growth from approximately $-0.8^{\circ}$ to nearly $-1.4^{\circ} \mathrm{C}$. Average ice thickness, as estimated from dip net samples measured on the ship in two different locations, was $1.1 \mathrm{~cm}$ at 22:00 on 26th Oct and $4.2 \mathrm{~cm} 3.5$ hours later. These estimates are averages from six and three samples with standard deviation of 0.12 and $0.29 \mathrm{~cm}$, respectively.
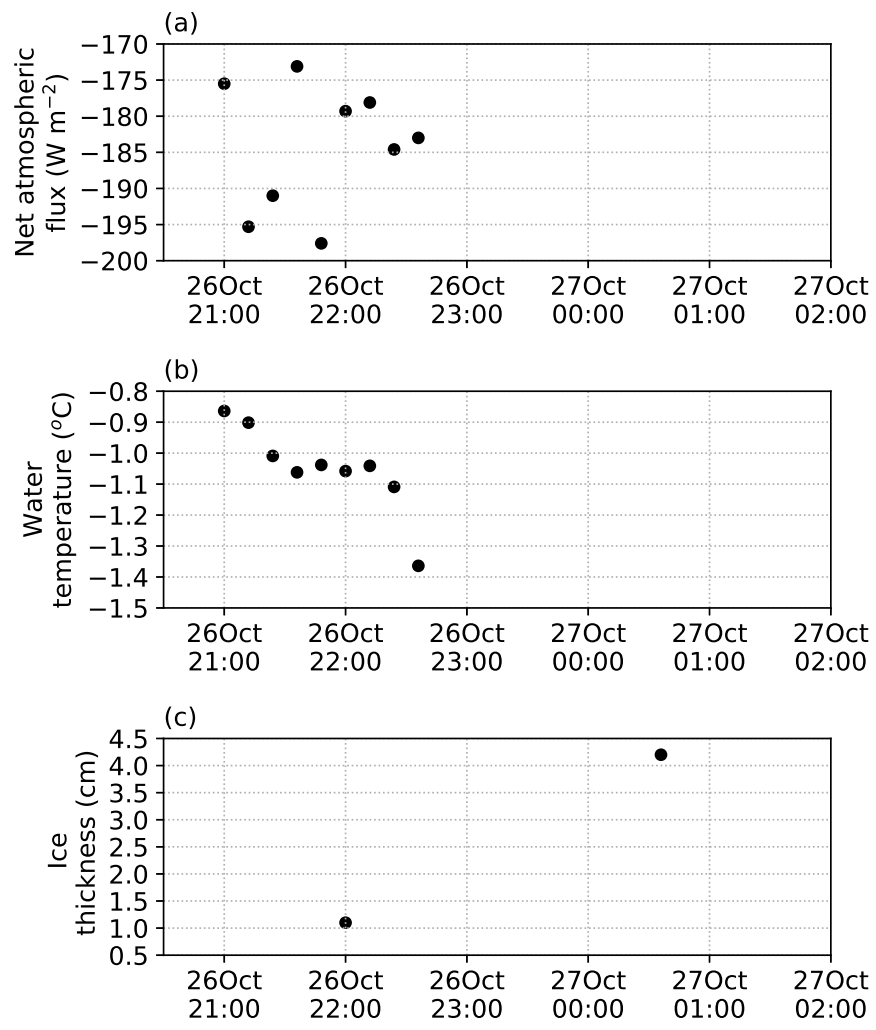

Figure 5.5: (a) Net atmospheric heat fluxes $\left(Q_{n e t}\right)$, (b) sea surface temperature, and (c) ice thickness measured from the ship within $10 \mathrm{~km}$ of buoy locations. 
Fig. 5.6 shows representative areas of floe components together with air temperature at a height of $1 \mathrm{~m}$ and significant wave height recorded by each of the buoys. There are strong negative correlations between the representative area of floe components and air temperature (Pearson correlation coefficients -0.71 for SWIFT09 and -0.96 for SWIFT14, both $p<1 \%$ ), with floes increasing in size as the air temperature cools (Fig. 5.6a-b). Correlations between the representative area and the water temperature $0.5 \mathrm{~m}$ below the surface were weaker. Water temperatures from this depth, which are above its freezing point, may not be a good indicator of mechanisms at the surface, which is subject to much colder conditions. A significant decrease in wave height was recorded by the SWIFTs during the deployment period (Fig. 5.6c-d). Lateral growth is strongly negatively correlated to significant wave height for both buoys (Pearson correlation coefficients -0.71 for SWIFT09 and -0.90 for SWIFT14, both $p<1 \%$ ). Lead/lag analysis (not shown) provided some indication that changes in significant wave height caused changes in floe area but this was not conclusive.

There is also some correspondence between floe welding and significant wave height (Fig. 5.7). For each image, the difference between the number of floe components and the number of discrete floes tells us the number of binary floe welding events that must have occurred, i.e. the the number of floe welds. The frequency of welded floes (i.e. the number of floe welds observed per number of discrete floes) increases for the whole period for SWIFT09 and between 22:00 and 01:00 UTC for SWIFT14. The frequency of welded floes is negatively correlated with significant wave height for SWIFT09 (Pearson correlation coefficient $-0.71, p<1 \%$ ). A positive feedback loop may be at play here: as floes get larger, they may damp waves more efficiently (Kohout and Meylan, 2008), and the resulting calmer sea state allows floes to freeze together into larger floes. 

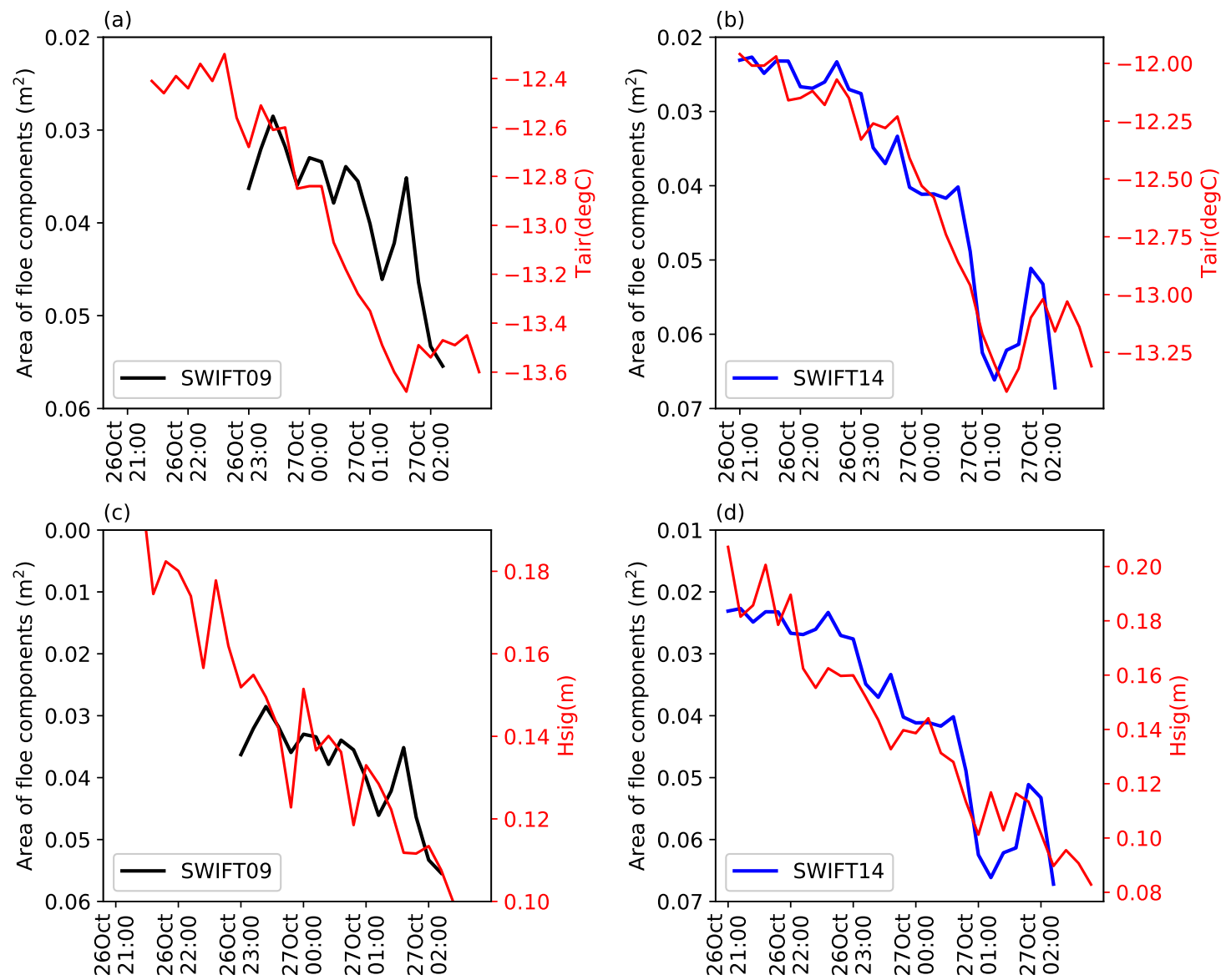

Figure 5.6: The evolution of the representative area of floe components over the deployment period for (a, c) SWIFT09 and (b, d) SWIFT14 shown with (a, b) air temperature (Tair) at a height of $1 \mathrm{~m}$ and (c, d) significant wave height (Hsig). Note that the y-axis for floe areas is inverted for all subplots and scales differ between $(a, b)$ and $(c, d)$ to facilitate comparison with decreasing air temperature and wave heights.
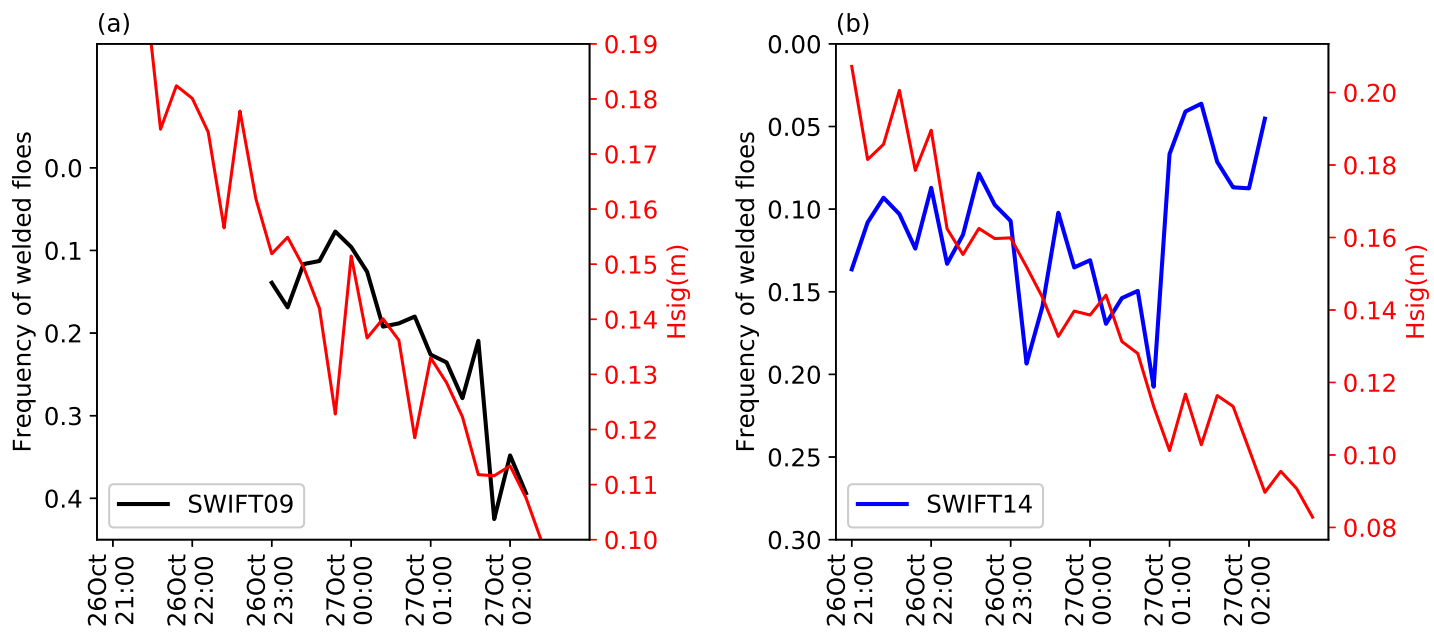

Figure 5.7: The frequency of welded floes (i.e. the number of floe welds observed per number of discrete floes) shown with (red axis) significant wave height over the deployment period for (a) SWIFT09 and (b) SWIFT14. Note the inverted axis for frequency of welded floes. 


\subsection{Wave constraints on floe growth}

Pancake sea ice floes form in the presence of surface waves, and the size of pancake floes and rate of growth depend on the wave dynamics. Prior theoretical study (Shen et al., 2001) suggested that maximum pancake size may be limited by either the tensile or bending stress mode resulting from the wave field. The tensile stress mode is caused by differential wave force which causes 'stretching' of surface between floes. Thus, assuming the ice-ocean drag coefficient is negligible, floes are limited in diameter by tensile failure according to

$$
D_{\max } \approx \sqrt{\frac{2 C_{2} \lambda^{2}}{\pi^{3} W_{A} g \rho_{\mathrm{ice}}}} \propto \sqrt{\frac{\lambda^{2}}{W_{A}}} .
$$

Here, $\lambda$ is the wavelength, $W_{A}=H_{s} / 2$ is the wave amplitude, $g$ is gravitational acceleration and $\rho_{\text {ice }}$ is ice density. $C_{2}$ is the tensile stress mode parameter, which is determined by local conditions and expected to be a function of temperature and salinity. The product of $C_{2}$ with ice thickness and floe diameter, $C_{2} D h$, is the force due to freezing between floes. Alternatively, growth of pancakes may be limited by the bending stress mode, where vertical tensile force creates bending force on floes. For bending failure, floe diameter is limited according to

$$
D_{\max } \approx \frac{C_{1} \lambda^{2}}{2 \pi^{2} E W_{A}} \propto \frac{\lambda^{2}}{W_{A}} .
$$

$C_{1}$ is a constant bending stress mode parameter, and $\mathrm{E}$ is the Young's modulus of the ice

floe. In the laboratory, Shen (2004) found that the maximum pancake size, $D_{\max }$, was proportional to the tensile stress mode rather than the bending stress mode.

Fig. 5.8 shows equivalent floe diameter computed from the representative area of discrete floes, $\bar{A}_{d}$, against the tensile and bending stress mode based on wave parameters measured by SWIFTs. Wavelength is estimated from peak period using the linear finitedepth dispersion relation, solved by Newton-Raphson iteration method with depth of approximately $4000 \mathrm{~m}$. There is a strong correlation of equivalent floe diameter with both the tensile stress mode $\left(R^{2}=0.76, p<1 \%\right)$ and the bending stress mode $\left(R^{2}=0.74\right.$, $p<1 \%)$. The linear fit in Fig. 5.8a gives an estimate of the tensile stress mode parameter of $C_{2}=0.167 \mathrm{~kg} \mathrm{~m}^{-1} \mathrm{~s}^{-2}$, which is close to the estimate of $C_{2}=0.118 \mathrm{~kg} \mathrm{~m}^{-1}$ $\mathrm{s}^{-2}$ observed by Shen (2004) in the laboratory. We note that in their study data from both urea-doped water and saltwater (35 psu) were used. 

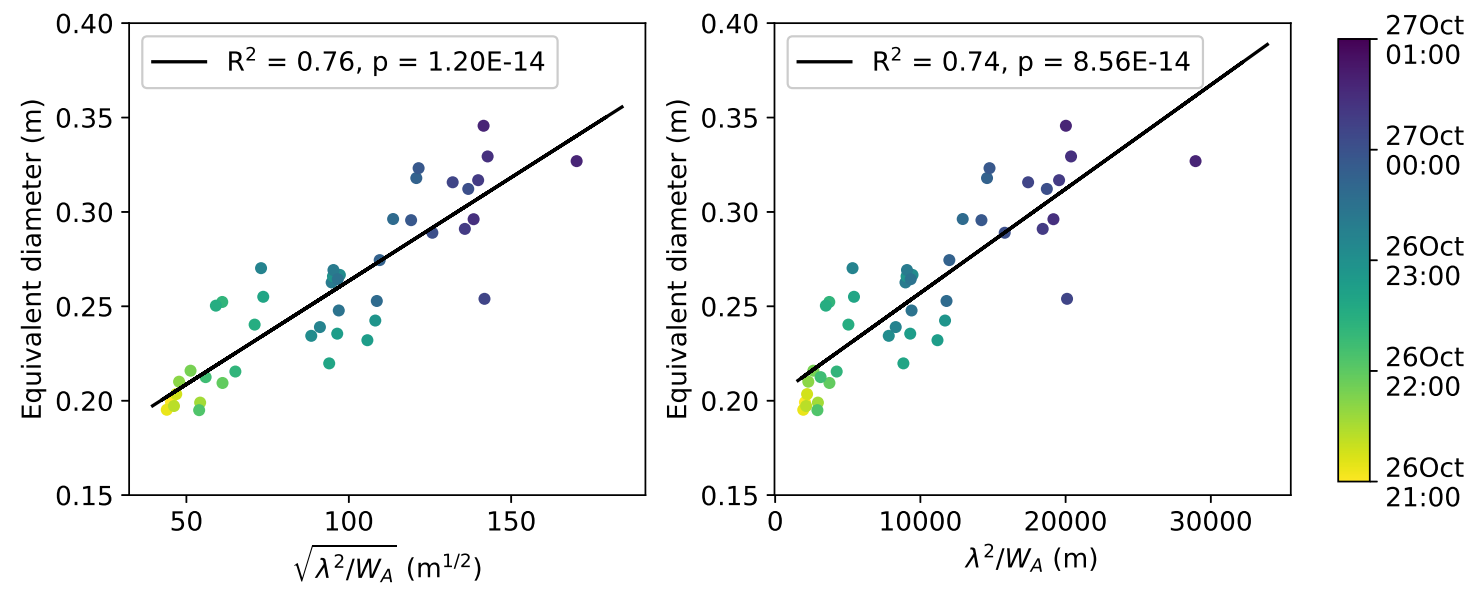

Figure 5.8: The equivalent diameter of discrete floes against (a) the tensile stress mode and (b) the bending mode calculated from SWIFT buoy wave measurements, with points coloured by time. Regression statistics are noted in the legend. Data from both SWIFTs are included.

These results indicate that waves are a dominant control on pancake ice growth, via the ratio of wavelength squared with amplitude $\left(\frac{\lambda^{2}}{W_{A}}\right)$. This provides strong support for the theoretical and laboratory results of Shen et al. (2001) and Shen (2004). However, our data are not sufficient to determine which of the two mechanisms proposed in Shen et al. (2001) dominate in these field conditions.

The representative diameter is proportional to the tensile and bending stress modes throughout the observed period of pancake ice formation (Fig. 5.8). This is due to the feedback between the change in wave conditions with an evolving ice field. Thin ice tends to damp high frequency waves, leading to a decrease in amplitude but an increase in peak wavelength. Under the tensile and bending stress theories, these conditions correspond to larger floe sizes (Shen, 2004) which may contribute to more efficient damping of waves (Kohout and Meylan, 2008).

The relationship between pancake diameter and tensile and bending stress modes is expected to break down as floe diameters increase and wave amplitude decreases, and floe welding begins to take over. The good fit of the stress modes through time (Fig. 5.8) suggests that this transition does not occur within the measurements presented here, although some welding is observed throughout the observation period. 


\subsection{Floe welding}

Roach et al. (2018b) propose that sea ice floes may merge or weld together during freezing conditions according to the geometric probability that two randomly placed floes overlap, in the absence of ocean surface waves. To describe this, they use a coagulation equation. The evolution of floe number per unit area, $\mathcal{N}$, is then proportional to concentration squared,

$$
\frac{\partial \mathcal{N}}{\partial t}=-\frac{\kappa}{2} C^{2}
$$

where the rate per unit area $\kappa$ is the total number of floes that weld with another, per square meter, per unit time, in the case of a fully covered ice surface $(C=1)$. The square in concentration arises as the geometric probability of overlap is the product of two area fractions; see Roach et al. (2018b) for further details. Although $\kappa$ may depend on surface temperature and the wave field, to implement this scheme in a large-scale model Roach et al. (2018b) assume $\kappa$ is constant.

Here, we calculate a value for $\kappa$, with the goodness of the linear fit showing how well the model with constant $\kappa$ describes observed floe welding. By our definitions, the change in the total number of floes due to welding per unit area over some time period is simply equal to the difference between the number of components $N_{c}$ and the number of discrete floes $N_{d}$ (which is the number of welds) per unit area, i.e.

$$
\frac{\Delta \mathcal{N}_{T}^{\text {weld }}}{\Delta t}=\frac{1}{a} \frac{N_{c}-N_{d}}{\Delta t}=-\frac{\kappa}{2} C^{2}
$$

where $a$ is the area of the image.

Fig. 5.9 shows the number of welds per unit area plotted against concentration squared for each image burst. We find a linear fit to these data, with correlation coefficient $R^{2}=0.51(p<1 \%)$. This provides support for the Roach et al. (2018b) floe welding model using a constant value for $\kappa$.

In a global sea ice model, $\kappa$ should represent the average for various conditions. Combining data from the two buoy deployments gives an approximate fit to the two different data sets, and the slope of the fit can be used to give an approximate order-of-magnitude estimate for $\kappa$. We expect scatter in the observed relationship, as the model neglects de- 


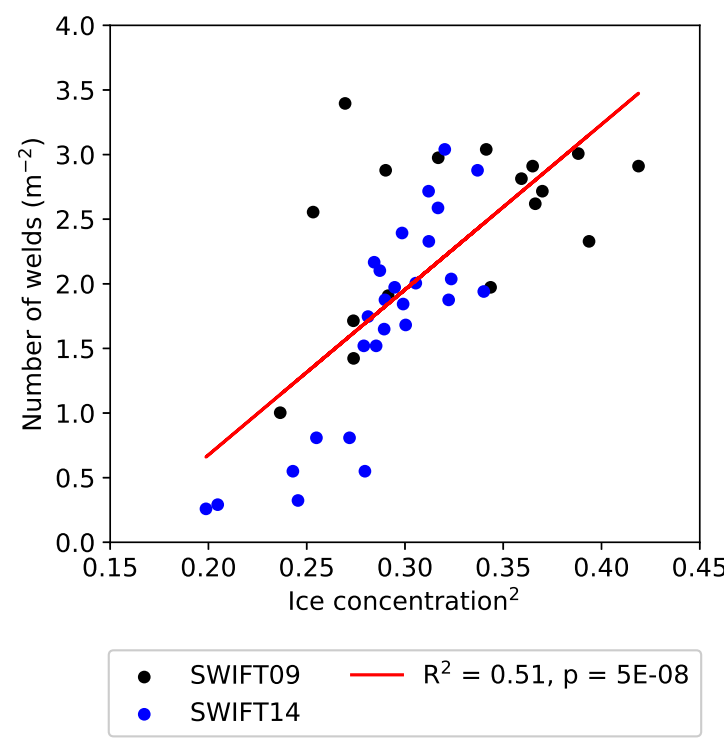

Figure 5.9: The number of floe welds per unit area against ice concentration squared, where the linear fit gives the coefficient of proportionality and regression statistics are noted in the legend. Data from both SWIFTs are included.

pendence of welding on sea surface temperature and wave conditions. Although the buoys were always less than $1 \mathrm{~km}$ apart, they may have experienced different local conditions which influenced the welding rate, such as winds or heat fluxes.

From Eqn. 5.6, the slope of the linear fit in Fig. 5.9 is equal to $-\frac{1}{2} \kappa \Delta t$. We are unable to discern the exact time period over which welding occurred, $\Delta t$, from the images. Ship observers noted that consolidation of frazil in pancakes was visible from 21:00 UTC for SWIFT09 and from 19:00 for SWIFT14. This occurred two hours before data from the image processing is available. Data from the images is available over five hours for SWIFT14 and three hours for SWIFT09. Therefore, the maximum value that $\Delta t$ could have is seven hours for SWIFT14 and five hours for SWIFT09. Taking $\Delta t=7$ hours, we find a minimum value of $\kappa=0.001 \mathrm{~m}^{-2} \mathrm{~s}^{-1}$. The choice of $\Delta t=7$ hours likely overestimates the time period, implying that the minimum value of $\kappa$ given here is a conservative estimate. This calculation can be used to inform the parameter value choice for floe welding in future models for sea ice floe size evolution. 


\subsection{Lateral growth of floes}

In this section, we approximately calculate what the Horvat and Tziperman (2015) model for lateral growth would predict and compare to data calculated from the SWIFT images. A more precise calculation requires a time series of sea ice thickness and net atmospheric flux at the ocean surface. Only a limited number of observations of these variables are available over our study period (Fig. 5.5), so we assume that sea ice thickness and net atmospheric flux are constant. As we use a constant floe thickness, the notation in this section differs slightly from Horvat and Tziperman (2015).

Horvat and Tziperman (2015) suggest that a lateral growth rate can be obtained from a net flux balance, with heat fluxes near ice contributing to lateral and vertical growth of existing floes. The partitioning between the contribution of a 'near-ice' heat flux, $Q_{\text {lead }}$, to lateral and vertical growth depends on the ratio of the basal surface areas to lateral surface areas. Let $N(r) \mathrm{d} r$ denote the number of floes per unit area with an equivalent radius between $r$ and $r+\mathrm{d} r$. Then the area of the vertical edges of the floes, per unit ocean area, is given by

$$
A_{\text {lat }}=\int N(r) \frac{2 h}{r} \mathrm{~d} r=\overline{(2 h / r)},
$$

where $h$ is the ice thickness. The contribution of the near-ice heat flux to lateral growth, $Q_{l, l}$, is

$$
Q_{l, l}=Q_{\text {lead }}\left(\frac{A_{\text {lat }}}{A_{\text {lat }}+C}\right) .
$$

The lateral growth rate, $G_{r}$ is then

$$
G_{r}=-\frac{Q_{l, l}}{q}
$$

where $q$ is the enthalpy.

The floe size distribution, $f(r) \mathrm{d} r$, is the area per unit ocean surface area covered by floes with equivalent radius between $r$ and $r+\mathrm{d} r$. Following Horvat and Tziperman (2015), the time evolution of the floe size distribution is given by

$$
\frac{\mathrm{d} f(r)}{\mathrm{d} t}=-\nabla_{r} \cdot\left[f(r) G_{r}\right]+\frac{2}{r} f(r) G_{r}
$$

Here, we evolve the initially-observed floe size distribution using this lateral growth 
scheme. Initial results indicated that using Eqn. 5.8 to partition the heat flux, even with the maximum ship-based dip net measurement of sea ice thickness in Eqn. 5.7, strongly underestimates the lateral growth rate. We therefore choose conditions that maximize the calculated rate, assuming that net heat flux from the ocean to the atmosphere is constant and given by the maximum ship-based estimate of net heat flux, $Q_{\text {lead }}=200 \mathrm{~W} \mathrm{~m}^{-2}$ (Fig. 5.5a). We further assume that all heat flux is used in lateral growth, $Q_{\text {lead }}=Q_{l, l}$, as the dynamic conditions and thin ice suggest that heat exchange would not be limited to the space between pancakes.

We calculate an equivalent radius, $r$, from the areas of floe components, $A_{c}$. Floe size categories are chosen by binning all observed floe radii from both SWIFTs over the whole deployment period into 12 equally-spaced categories. The initial floe size distribution, $f(r, 0) \mathrm{d} r$, is the area per unit ocean surface occupied by floes of size $r$ to $r+\mathrm{d} r$. After time $\Delta t$, the floe size distribution is

$$
f(r, t) \mathrm{d} r=f(r, 0) \mathrm{d} r+G_{r} \Delta t\left[-\frac{\mathrm{d} f(r, 0)}{\mathrm{d} r}+\frac{2}{r} f(r, 0)\right] \mathrm{d} r
$$

and the evolution of the ice concentration, $C(r, t)=\int f(r, t) \mathrm{d} r$, is given by

$$
C(r, t)=C(r, 0)-G_{r} \Delta t \int \frac{2}{r} f(r, 0) \mathrm{d} r
$$

These equations are evolved with $\Delta t=12$ mins. The evolution of the representative floe area is given by the evolution of the total ice area divided by the initial number of floe components.

In order to sample time variability, which is the largest error source in the area observations, we repeat the calculation using initial conditions from each one of the ten images from the initial burst. We show the mean of the ten calculations in Fig. 5.10 using floe areas from the first user trial, with the standard deviation across the initial condition ensemble. This is compared to the observed representative area of floe components and its time variability. We find some validation for the Horvat and Tziperman (2015) model, as the value ranges from model and observations overlap for SWIFT09 and for the first two hours of SWIFT14, but they diverge for the rest of the deployment period for SWIFT14. We are unable to conclude what conditions led the model to perform better in comparison to observations from one buoy than the other. 

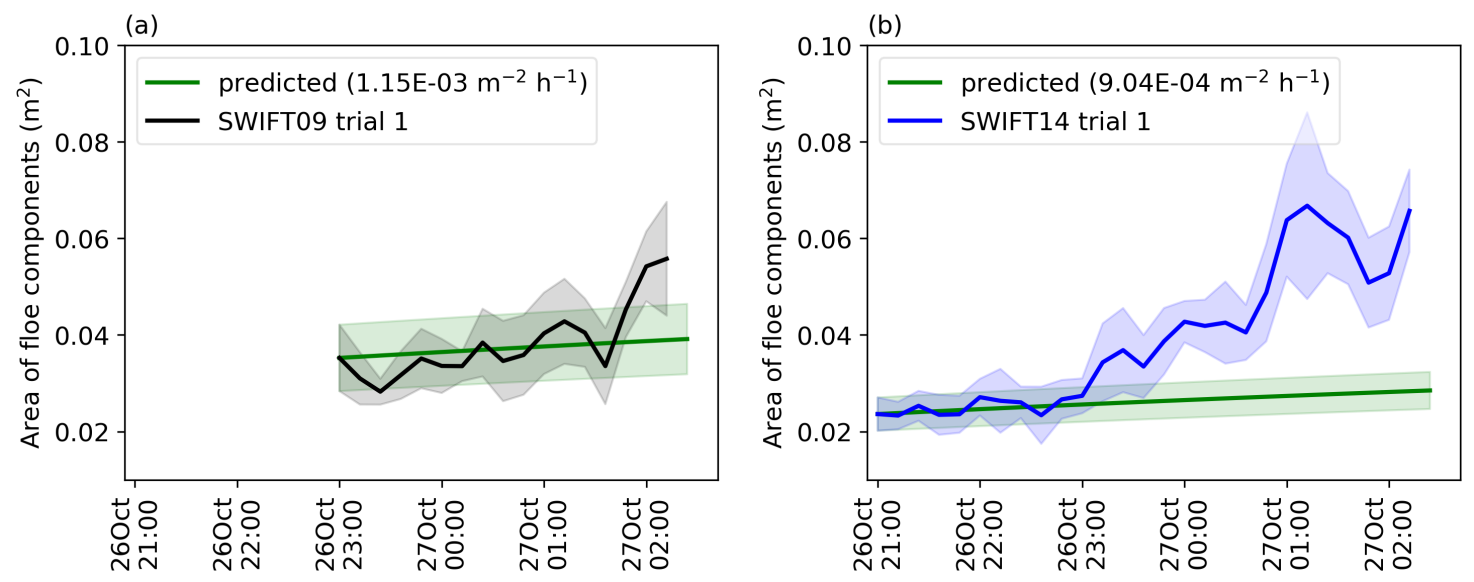

Figure 5.10: The representative area of floe components from SWIFT images (black), where grey shading indicates the standard deviation across sub-burst time variability. Also shown are the areas predicted by the Horvat and Tziperman (2015) lateral growth model, initialized from the mean properties of the first burst, with green shading indicating the evolution initialized from the range of initial conditions shown by the grey shading. The legend includes the predicted linear lateral growth rate. Both use floe areas computed in the first user trial from (a) SWIFT09 images and (b) SWIFT14 images.

A constant flux is used to force the model, resulting in a linearly increasing floe area, with a rate of $1.1 \times 10^{-3} \mathrm{~m}^{2} \mathrm{~h}^{-1}$ for SWIFT09 and $0.9 \times 10-3 \mathrm{~m}^{2} \mathrm{~h}^{-1}$ for SWIFT14. The calculated rates are the same using floe areas from the first and second user trials (to two significant figures). A linear fit to the observed floe areas gives a lateral growth rate of $6.1 \times 10^{-3} \mathrm{~m}^{2} \mathrm{~h}^{-1}\left(R^{2}=0.65\right)$ for SWIFT09 and $8.6 \times 10^{-3} \mathrm{~m}^{2} \mathrm{~h}^{-1}\left(R^{2}=0.84\right)$ for

SWIFT14. Predicted lateral growth rates are thus five and nine times smaller than those observed, in spite of our choices to maximize the lateral growth rate. However, the modelled rates are of a similar order of magnitude to observations, providing some support for the Horvat and Tziperman (2015) model.

\subsection{Discussion}

In this study, we compared observations with three theoretical models for determining floe size. The three models describe different physical processes-wave constraints on sea ice growth, floe welding, and lateral growth-all of which would be required in a comprehensive sea ice model appropriate for the marginal ice zone. Here we discuss the suitability of the models for the observed conditions and implications of this study for future work. 
Very good agreement with observations of sea ice growth is found using the Shen (2004) model, where stresses arising from wave force control the maximum floe size (Fig. 5.8). The strong negative correlation of wave height with floe area (Fig. 5.6) provides qualitative support for this model. The Shen (2004) model is the only model examined here that explicitly includes wave information, and as such is best suited to the conditions observed. The relationship between pancake diameter and tensile and bending stress modes is expected to break down as floe diameters increase and wave amplitude decreases. The increase in welded floes over time observed by one SWIFT (Fig. 5.7a) provides some indication that tensile and bending stress between floes are decreasing, such that floes can merge together. However, we do not see a transition from floe size being determined principally from wave activity to being determined by lateral welding of pancakes in the measurements presented here. Determining when this transition occurs could be useful for floe size evolution in future wave-ice coupled models. This would require observation into more quiescent wave conditions. It is worth nothing that the Shen (2004) model may be of limited use for development of models of the sea ice floe size distribution, as it only predicts a single floe size. This model could instead be used to set the initial size of floes formed in a wave field.

Observations show good agreement with the relationship between floe welding and concentration arising from geometric probability, as proposed by Roach et al. (2018b). The observed relationship can be used to give an order-of-magnitude estimate of a key model parameter that determines the rate of floe welding and is highly relevant for sea ice evolution (Roach et al., 2018b). This study represents the first attempt to empirically quantify the floe welding rate. However, the size of floes observed is relatively small from a climate model perspective (although close to the smallest floe size category in Roach et al. 2018b), and larger floes may exhibit different welding behaviour. Additional field studies with a range of environmental conditions and floe sizes would give a better constraint on the welding parameter. We also note that the welding parameter is likely to depend on a variety of environmental factors, with relationships that may be difficult to determine from field measurements. Laboratory experiments, where different environmental variables can be controlled, would help unravel relationships with wave parameters and surface temperature. 
Predictions based on the Horvat and Tziperman (2015) model, which uses ocean freezing potential to describe evolution of floe size distribution under lateral growth, show some overlap with observed floe area values, particularly for one buoy. Modelled growth rates are of the correct order of magnitude, but are smaller than those observed. This is despite the choice of variables to maximize the growth rate, with model predictions made using net atmospheric flux as the ocean freezing potential, which we assumed to be constant and to be used only for lateral growth. There are several possible explanations for the difference between observations and the model calculation. Firstly, the Horvat and Tziperman (2015) model is designed to describe the transition from open water to solid ice, without resolving grease ice or crystal accumulation, processes which may be important on the short timescales observed in this study. Secondly, the flux in the model calculation was assumed constant, using a measurement taken after the formation of frazil crystals; better agreement with observations may be obtained using a time series of flux measurements. Thirdly, it is possible that our analysis overestimates the observed lateral growth rate. Ice area estimates (e.g. Fig. 5.1c) exclude loose frazil crystals on the edge of sea ice floes as they are not solid ice. However, if the ice thickens, there may be additional loose frazil crystals at depth. If this occurs, the edges of floes may become whiter and be counted as solid ice, thereby misrepresenting thickness growth as lateral growth. Further, we assumed that floe welding conserves ice area which, if untrue, may also introduce spurious lateral growth into the analysis. We are unable to determine whether these effects occur with the available data.

This study demonstrates a novel application of image processing, which allows us to isolate individual sea ice processes using images from a near-Lagrangian platform. The methods used here could be applied to existing sets of images of floe growth to obtain growth and welding time scales under different ice and atmospheric conditions, providing that floe size and surface characteristics were sufficiently uniform. For less uniform characteristics, as expected with older sea ice floes, a similar analysis could be applied using improved image processing techniques, and/or fully-Lagrangian tracking of sea ice floes. Such future observational campaigns would greatly assist modelling efforts. We additionally encourage studies on other isolated floe size dependent processes, such as lateral melt, which is also poorly constrained and has a large effect on sea ice concentration (Roach et al., 2018a). 


\subsection{Conclusions}

This study analyses images captured by drifting buoys, which show the evolution of small pancake floes in dynamic ocean conditions. The floes grow laterally and weld together to form composite floes, two processes which are quantified using image processing techniques. Lateral growth has a much greater contribution to floe size than welding during the study period. Both processes correlate with significant wave height, underlining the tightly coupled nature of wind, waves, and sea ice growth in the marginal ice zone.

The results describe three physical processes which are required for models describing sea ice floe size. We find that the Shen et al. (2001) model for pancake ice formation describes floe size well in these conditions. Although they neglect wave activity and small-scale frazil processes, the Roach et al. (2018b) model for floe welding and Horvat and Tziperman (2015) model for lateral growth capture some of the observed behaviour. We obtain an estimate for a constant floe welding parameter, which can be used for model development.

The observations presented here quantify freezing processes at the floe scale in situ for the first time. New developments in sea ice modelling which resolve small-scale, floesize-dependent processes (e.g. Horvat and Tziperman 2015, Zhang et al. 2016, Bennetts et al. 2017, Roach et al. 2018b) require such process-based observations to constrain parameters and validate results, which have significant impacts on simulated sea ice. 


\section{Article citation}

Roach, L. A., M. M. Smith, and S. M. Dean (2018c), Quantifying growth of pancake sea ice floes using images from drifting buoys, Journal of Geophysical Research: Oceans, 123(4), 2851-2866, doi:10.1002/2017JC013693

\section{Acknowledgements}

SWIFT observations were funded by ONR award N00014-13-1-0284. The data used are available from the Sea State collaboratory under the Data tab at www. apl. uw.edu/ arcticseastate. L. Roach and S. Dean were funded via Marsden contract VUW-1408. The authors wish to thank the crew of the $R / V$ Sikuliaq, Alex de Klerk and Joe Talbert for SWIFT support, Stephen Ackley for suggesting use of this data set, and M. Jeffrey Mei for assistance with initial image processing. We are grateful to Hayley Shen, Jim Thomson, Chris Horvat and two anonymous reviewers for comments on the manuscript. 


\section{Chapter 6}

\section{Variability in floe size distribution simulated by a model with improved representation of wave-limited floe sizes}

In Chapter 5, we found evidence to support use of a new relationship based on the ocean wave field to describe the sizes of sea ice floes when they form for the first time. In this chapter, I update the model from Chapter 4 to include this new scheme for ice growth and present spatial, seasonal and inter-annual variability in floe size using the updated model in both climatological and historical simulations. I consider trends in floe size in the Arctic due to the recent rapid decline in Arctic sea ice cover. I also investigate the impacts of including floe-size-dependent lateral melt using the updated model on sea ice concentration, to address a hypothesis posed in Chapter 3. The original research presented in this chapter is as-yet unpublished.

\subsection{Introduction}

Fragmentation and opening of polar sea ice cover permits heat transfer between the ocean and atmosphere, increases radiative heating of the ocean surface layer, lowers regional surface albedo, and allows wave-generation and wind-driven ocean mixing. These processes impact local weather and climate, making prediction of sea ice fragmentation relevant for weather and climate prediction. Besides this, predictions of sea ice fragmentation are useful to polar stakeholders, whether they be indigenous communities who use sea ice for transportation, engineers designing structures that must withstand loading by sea ice, 
or ship operators navigating the polar seas.

Sea ice can be fragmented if it melts into small pieces, is broken up by ocean surface waves, or is prevented by waves from forming extensive sheet ice during freeze-up. The degree of fragmentation and how it leads to enhanced melt can be described using the floe size distribution (FSD), the distribution of differently-sized discrete pieces of sea ice. The FSD varies widely across space and time under forcing from many different physical processes. Chapter 4 presented a process-based model that captures some key characteristics of the FSD. This is the first global model to describe a prognostic FSD that emerges due to different physical processes.

Here, we build upon this earlier work to further investigate the spatial and temporal variability of the sea ice floe size distribution. We argue for use of the total floe perimeter as a metric to quantify FSD evolution. Motivated by Chapter 5, we implement a scheme calculating the sizes of new floes based on the properties of the ocean surface wave field. We then investigate spatial and seasonal variability in the FSD simulated by the updated model using climatological forcing. Conducting transient historical simulations over the satellite era allows us to revisit the hypothesis posed by Chapter 3 that the floe size dependence of lateral melt is relevant for biases in the sea ice concentration distribution. Finally, these transient experiments can be used to gain insight on how rapid changes in Arctic climate may have driven changes in the sea ice floe size distribution, with implications for the future of Arctic sea ice cover.

\subsection{Methods}

\subsubsection{Metrics}

Chapter 4 showed process contributions to the number FSD for different floe sizes and to the representative radius in space and time. Recent modelling (Zhang et al., 2016) and observational (Stern et al., 2018a) studies have used the power law exponent to condense information on the FSD to a single number that can be shown in time and space. However, given the statistical issues facing observations of the number FSD (Stern et al., 2018b), and that the truncation of floe size categories in FSD models causes deviations from a power law (Chapter 4 and Appendix C), we recommend use of a metric that does not require assumptions as to the shape of distribution. 
Here, we propose use of the total floe perimeter as an additional metric for showing evolution of the FSD. Given that the area of a floe is $x(r)=4 \alpha r^{2}$ and the circumference of a floe is $8 \alpha r$, the perimeter of floes per unit area of ocean, $P_{o}$, is

$$
P_{o}=8 \alpha \int_{\mathcal{R}} r F^{N}(r) \mathrm{d} r
$$

where $F^{N}(r) \mathrm{d} r$ (units $\mathrm{m}^{-2}$ ) is the number of floes per unit ocean surface area with lateral floe size between $r$ and $r+\mathrm{d} r$.

This metric is relevant for climate modelling as it succinctly describes the susceptibility of the ice pack to lateral melt. The lateral growth and melt of floes at a rate $G_{r}$ is given by

$$
\mathcal{L}_{T}(r, h)=-\nabla_{(r)} \cdot\left(f(r, h) G_{r}\right)+\frac{2}{r} f(r, h) G_{r},
$$

where the first term describes movement of floes between lateral size classes and the second term describes the total ice area added or removed that belongs to floes of size $r$ (Horvat and Tziperman, 2015). Integrating Eqn. 6.2 over all floe sizes, the first term vanishes and the second term equals $P_{o} G_{r}$. The addition or removal of sea ice concentration via lateral thermodynamics therefore depends directly on the perimeter of floes per unit area of ocean.

Perimeter per unit area can intuitively be integrated over surface areas to obtain hemispheric quantities. It has been used in observational studies (Perovich, 2002; Perovich and Jones, 2014), but has not previously been used in FSD modelling studies. Perimeter can be measured directly from aerial images (Perovich, 2002; Perovich and Jones, 2014) and-as any visible floe perimeter can be included, even if the whole floe is not enclosed in the image - avoids some of the biases associated with constructing a size distribution from a small aerial image, making it a good candidate metric for model-observation comparisons. Further, it does not require assumptions on the shape of the FSD, and is a single number that can easily be shown in space and time.

\subsubsection{Model configuration}

We use the FSD model described in Chapter 4, implemented in CICE5 and coupled to the ocean model NEMO. The model is forced by the JRA55 atmospheric renalaysis and 
a Wavewatch hindcast, which was also forced by JRA55. All simulations described here are initialized from the 65-year-long climatological simulations described in Chapter 4 with and without a FSD. Simulations without a FSD are denoted 'STD.' Climatological simulations use repeated atmospheric and wave forcing corresponding to 1975 . The additional climatological simulations required for this chapter were all conducted for twenty years. Time series of global average representative radius suggested that the first ten years of the simulation were sufficient to allow adjustment to changes in physics, so the second ten years are used for analysis.

Transient simulations with atmospheric forcing that varies each year are conducted over 1975-2014 and initialized from the end of the corresponding climatological run. For the purposes of presenting results in this chapter, we use the period from 1983-2014 for analysis (see Appendix B).

\subsubsection{Wave-limited floe sizes}

In the first version of our model (FSD-v1, Chapter 4), all new ice is initially assigned to the smallest floe size category, representing formation of pancake ice, as this was hypothesised to be the dominant ice formation mechanism in the Antarctic. For comparison, neither Zhang et al. $(2015,2016)$ nor Bennetts et al. (2017) specify the sizes assigned to new ice. In reality, pancake floes form only in the presence of surface waves and/or winds; frazil crystals freeze together to form large thin sheets of sea ice called nilas in the absence of wind and wave action (Weeks and Ackley, 1986). Formation of sea ice by nilas may be an important mechanism in the Arctic.

The choice of floe size assigned to new floes strongly impacts the floe number distribution. Chapter 4 suggested using the wave field to differentiate between nilas and pancake growth following Shen et al. (2001), who proposed that maximum pancake size may be limited by either the tensile or bending stress mode resulting from the wave field. The tensile stress mode is caused by differential wave force which resulting in 'stretching' of the surface between floes, while the bending stress mode is caused by vertical tensile force. Shen (2004) performed laboratory experiments that suggested floe size is controlled by tensile rather than bending stress. The field observations presented in Chapter 5 provided further support for use of the tensile stress mode resulting from the wave field to calculate the initial sizes of floes. Here, we implement this suggestion in a second version of 
the FSD CICE scheme (FSD-v2) and investigate how this modifies simulation of the FSD.

Following Shen et al. (2001), assuming the ice-ocean drag coefficient is negligible, floes are limited in diameter by tensile failure according to

$$
D_{\max } \approx \sqrt{\frac{2 C_{2} \lambda^{2}}{\pi^{3} W_{A} g \rho_{i}}}
$$

Here, $\lambda$ is the wavelength, $W_{A}$ is the wave amplitude, $g$ is gravitational acceleration and $\rho_{i}$ is ice density. $C_{2}$ is the tensile stress mode parameter, which is determined by local conditions and expected to be a function of temperature and salinity. The product of $C_{2}$ with ice thickness and floe diameter, $C_{2} D h$, is the force due to freezing between floes.

In our updated model, new ice is assigned the floe size category corresponding to $D_{\max }$ as calculated from Eqn. 6.3. Wave parameters in Eqn. 6.3 are calculated from a wave spectrum in the grid cell where freezing occurs. We reconstruct the Bretschneider spectrum, $S_{B}(T)$, using significant wave height, $H_{s}$, and mean zero-crossing period, $T_{z}$, from a Wavewatch hindcast outside the ice. The spectrum as a function of period, $T$, is

$$
S_{B}(T)=\frac{1}{4 g} \frac{H_{s}^{2}}{T_{z}^{4}} T^{2} \exp ^{-\frac{1}{\pi}\left(\frac{T}{T_{z}}\right)^{4}} .
$$

and it is attenuated through sea ice according to the attenuation coefficient as a function of mean ice thickness and wave period from Horvat and Tziperman (2015) and the number of floes between the ice-covered cell and the cell where wave data is drawn from, $\sum n_{\mathrm{fl}}$ (see Chapter 4).

As frequency $f$ is $1 / T$, the spectrum as a function of frequency, $E(f)$, is

$$
E(f)=S_{B}(T)\left|\frac{\partial f}{\partial T}\right|=S_{B}(T) T^{-2}
$$

The wavelength $\lambda$ is calculated from the peak frequency $f_{p}$, using the deep-water surface gravity wave dispersion relation,

$$
\lambda=\frac{g}{2 \pi f_{p}^{2}}
$$

The peak frequency is the value of $f$ that maximizes $E(f)$. For consistency with Chapter 5, we assume that the wave amplitude is $W_{A}=H_{m 0} / 2$, where the spectral height 
parameter, $H_{m 0}$, is given by

$$
H_{m 0}=4 \int E(f) d f .
$$

We use the estimate of $C_{2}=0.167 \mathrm{~kg} \mathrm{~m}^{-1} \mathrm{~s}^{-2}$ from Chapter 5 .

\subsection{Results \& Discussion}

\subsubsection{Impact of wave-limited ice floe sizes}

We proceed by comparing the FSD from climatological simulations with (FSD-v2) and without (FSD-v1) the wave-limited floe size scheme at the hemispheric scale. To facilitate comparison with Chapter 4, we show the cell-average area-weighted representative floe radius for the two simulations in Fig. 6.1. The representative floe radius is significantly larger in FSD-v2 than FSD-v1 in the interior ice pack, with the impact of the additional wave-ice interaction physics being greater in the Arctic than the Antarctic. In the Arctic, the ice cover is now dominated by large floes. Small areas with low representative radii, which could be considered as marginal ice, are present on the edge of the ice pack in winter, and melt away during summer. In both simulations, floes are smaller in the Antarctic than the Arctic, but the maximum representative radii in the two hemispheres are closer together in FSD-v2 than FSD-v1.

Fig. 6.2a-d show total hemispheric number distributions for FSD-v2, which are obtained by integrating $F^{N}(r)$ over the ocean area in each hemisphere. The four total number distributions have a similar shape: decaying with increasing floe size until the largest floe size categories, which contain more floes, with bending at radii of around $100 \mathrm{~m}$. Truncation of the FSD through existence of a maximum floe size causes a build-up of floes at the largest size and during fall (NH September and SH March) at the next-tolargest size as well. Compared to the equivalent figure for the FSD-v1 run (Fig. 4.2), FSD-v2 has more floes in the largest floe size category and fewer floes in the smallest floe size category, as floes are initialized at larger sizes and subsequently survive. The slope of the distribution on a log-log plot for floe size below $100 \mathrm{~m}$ is flatter in the FSD-v2 simulation than the FSD-v1 simulation, and the bending around $100 \mathrm{~m}$ is more apparent. The distributions are less well-characterized by straight lines on a log-log plot than those from the FSD-v1 simulation. 
(a) $\mathrm{NH} \mathrm{Mar}$

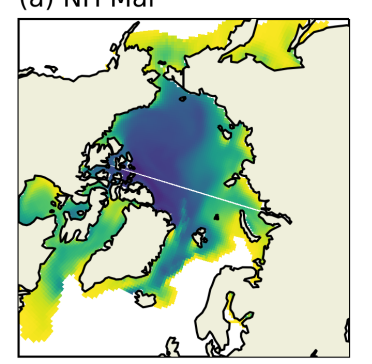

(b) $\mathrm{NH}$ Sep

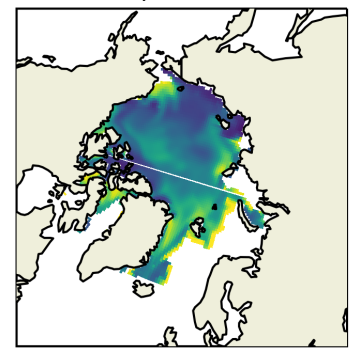

(c) $\mathrm{SH}$ Mar

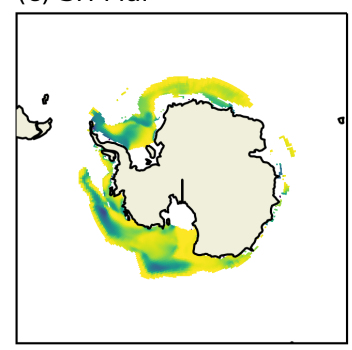

(d) SH Sep

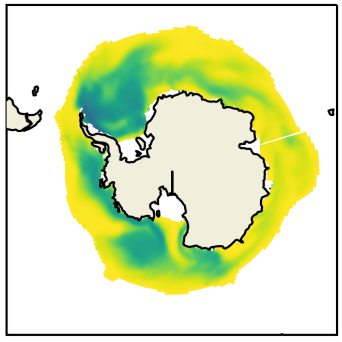

(h) SH Sep
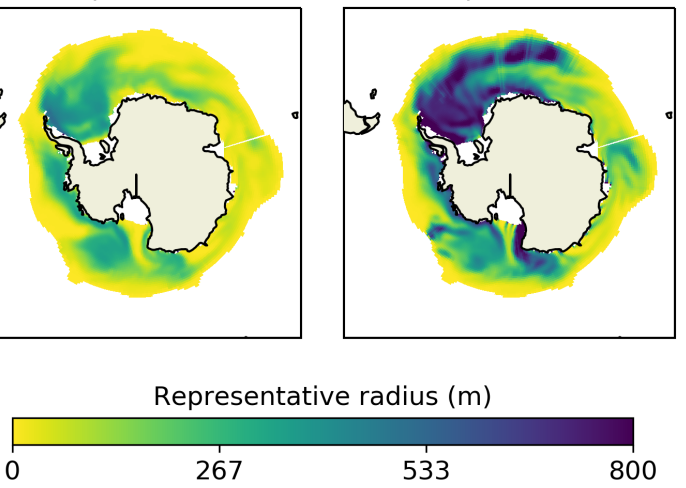

Figure 6.1: The representative radius averaged over the final ten years of (a-d) the FSD-v1 and (e-h) the FSD-v2 climatological runs. The left column is identical to Fig. 4.1, but with a modified colour scale. 
As in Fig. 4.2, Fig. 6.2e-h show the tendencies arising from different floe processes in the total hemispheric number distributions from FSD-v2, where the tendency in the number FSD due to some process is defined as

$$
\frac{\mathrm{d} F^{N}(r)}{\mathrm{d} t}=\frac{1}{\mathrm{~d} t}\left(F(r)_{\mathrm{after} \text { process }}^{N}-F(r)_{\mathrm{before} \text { process }}^{N}\right)
$$

and the model monthly output is the time average of $\frac{\mathrm{d} F(r)}{\mathrm{d} t}$. New ice is created at wavedetermined floe sizes in all floe size categories. The tendency in the number distribution arising from new ice growth follows a straight line in log-log space, until the largest floe size category, for both hemispheres and months shown. When there is no wave information in a grid cell, new ice is formed at the size of the largest floe size category. If extended, the tendency in number FSD arising from new ice growth may tend to zero with increasing floe size. Alternatively, introduction of wind effects on the sea surface within the sea ice region could result in small but non-zero values for wave properties and reduce the occurrence of very large floe sizes. That formation of new ice itself may generate power-law-like behaviour in the FSD has not previously been suggested.

There are some modifications to other process impacts with the addition of wavedependence to new ice growth. The net tendency in the number distribution arising from floe welding differs substantially from the FSD-v1 run, which was dominated by the welding together of floes in the smallest floe size category. The net tendency arising from wave fracture also differs due to the higher number of very large floes in the FSD-v2 run which can be broken. The tendency arising from wave fracture is smoothed relative to FSD-v1, most notably in the SH in September. This points to wave fracture redistributing floes to all lower sizes below some critical floe size, and away from the idea of 'preferred sizes' noted in Chapter 4. The SH in March still exhibits alternating net positive and net negative tendencies in the number FSD arising from wave fracture at sizes below $100 \mathrm{~m}$, however. The tendencies arising from lateral growth and melt are broadly similar to FSDv1.

The wave-dependent sea ice growth scheme included here, describing formation of both pancakes and nilas, certainly improves the physical fidelity of the model. It increases floe sizes, resulting in a floe radius climatology that may be more plausible than the initial scheme. There is a more defined transition between pack ice and more fragmented ice. 

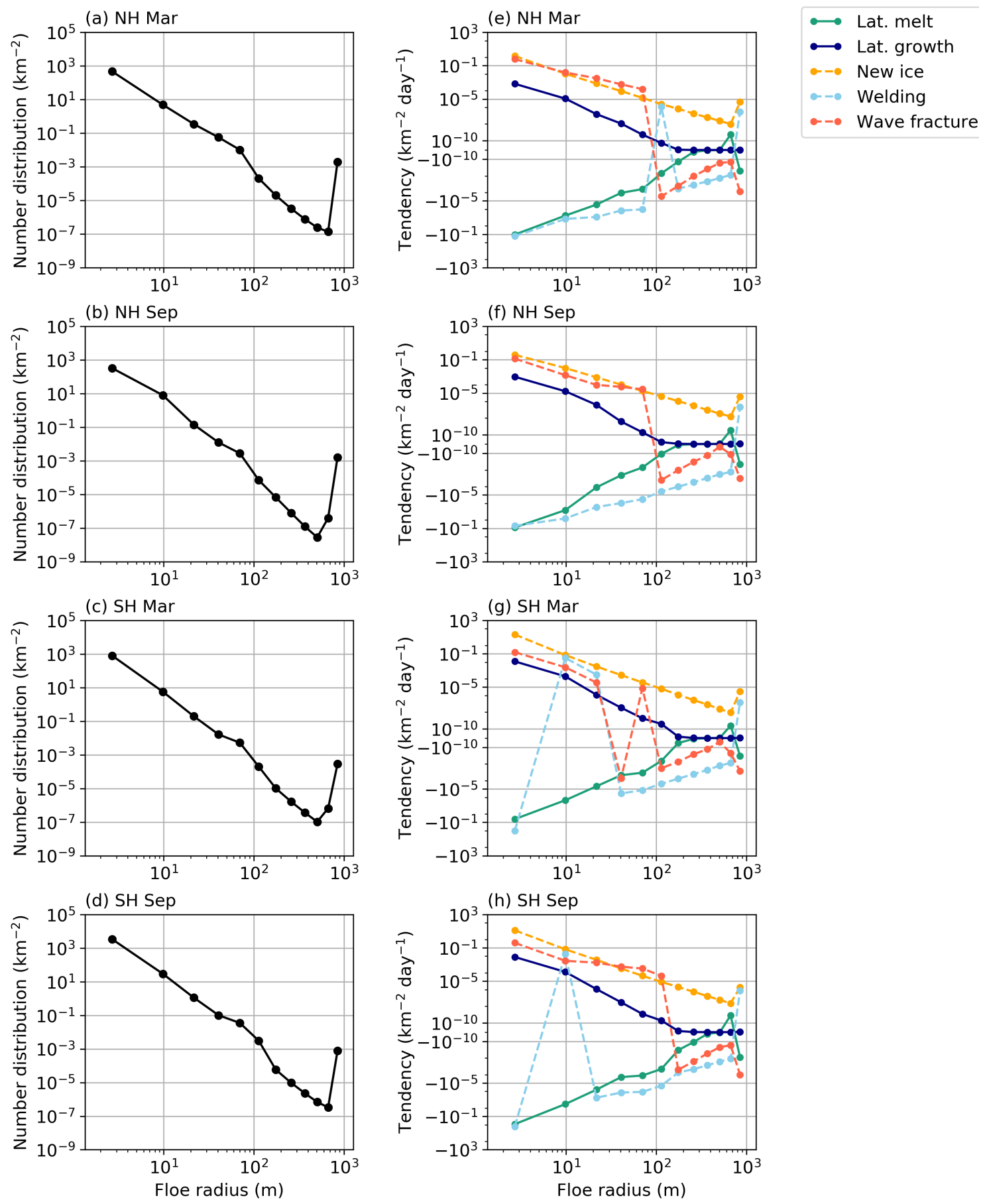

Figure 6.2: (a) The Northern Hemisphere floe number distribution from the FSD-v2 climatological simulation in March averaged over the ten years following model spin-up. (b-d) Same as (a), for the Northern Hemisphere in September, the Southern Hemisphere in March, and the Southern Hemisphere in September, respectively. (e) The net tendency in the floe number distribution from different physical processes in the Northern Hemisphere in March averaged over the twenty years following model spin-up. The axis in (e) is linearized around zero. (f-h) Same as (e), for the Northern Hemisphere in September, the Southern Hemisphere in March, and the Southern Hemisphere in September respectively. 

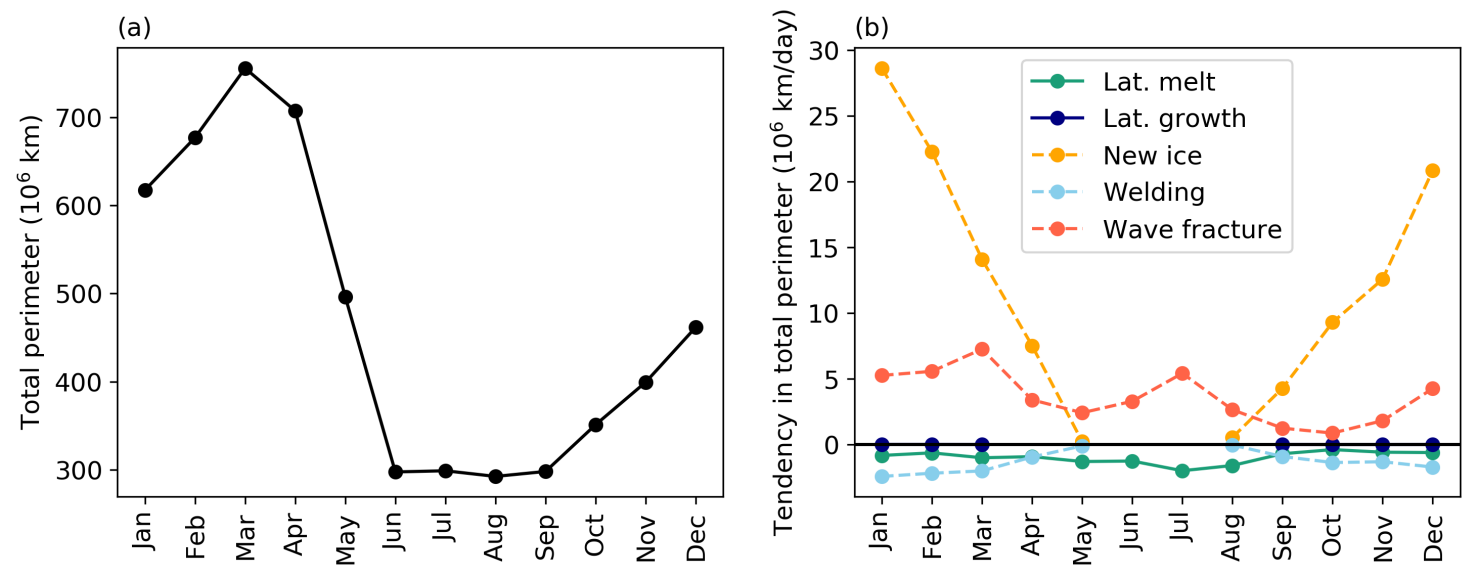

Figure 6.3: (a) The total perimeter of ice floes and (b) the average tendency in total perimeter arising from different physical processes, in the Northern Hemisphere from the FSD-v2 climatological simulation averaged over the ten years following model spin-up.

Lack of validation data sets preclude definitive evaluation. We note, as in Chapter 4, that the standard NEMO-CICE simulation without floes exhibits a low sea ice concentration bias in the Antarctic, reducing the likelihood of floe welding, and may result in low-biased SH floe sizes.

The addition of the new sea ice growth scheme increases the dependence of the FSD on ocean surface waves. As discussed in Chapter 4, our wave attenuation scheme is simplistic, including only ocean swell, without wind input to swell or wind-generated waves in the sea ice region. If included, these processes would act to decrease floe sizes through fracture and prevention of nilas growth. The area affected by ocean waves in the Arctic in our model is also strongly limited by the presence of the continents. Preliminary results from a coupled CICE-Wavewatch model-including the new sea ice growth scheme, permitting wind-driven wave generation, and less constrained by the continents than our model - show reduced floe sizes and increased marginal ice zone area relative to our results from FSD-v2 (pers. comm. C. Bitz). When waves are correctly included, the Arctic FSD may thus lie somewhere between the FSD-v1 and FSD-v2 runs described here.

\subsubsection{Seasonal evolution of Arctic floe perimeter}

To examine seasonal evolution of the FSD, we use the climatological FSD-v2 simulation incorporating wave-limited new ice floe sizes. The total NH floe perimeter from this simulation is shown in Fig. 6.3a. Note that changes in the total perimeter can occur through 
changes in concentration as well as changes in the FSD. For example, wave fracture will increase perimeter while concentration stays the same, while basal melt will decrease both perimeter and concentration if the sea ice thickness in a grid cell is reduced to zero. The seasonal cycle of floe perimeter is asymmetric: increasing slowly from September to a maximum in March, and dropping rapidly to the minimum in June, with little change until September. This is plausible: pancakes and smaller floes can be formed throughout winter while larger floes are fractured by waves, steadily increasing perimeter, and small floes are then rapidly removed by melt processes between April and June. This asymmetry could be tested with observations to determine whether the model is accurately capturing behaviour of the FSD. Perovich and Jones (2014) show floe perimeter on ten dates between June and September calculated from small aerial photographs taken during the 1997-1998 field campaign of the Surface Heat Budget of the Arctic program (SHEBA). If extended, this or a similar time series could be compared to model output.

Fig. 6.3b shows how FSD-dependent processes contribute to the seasonal cycle in total perimeter. With small impacts, floe welding reduces floe perimeter from September through to April and lateral melt reduces floe perimeter from May to August by removing small floes. The most important FSD-modifying processes for total perimeter are both related to ocean surface waves: wave-dependent new ice growth is the most significant apart from during May to August, when wave fracture is dominant. During May to August, changes in concentration due to lateral melt are largest. The tendencies in floe perimeter arising from different FSD-dependent physical processes therefore highlight the importance of wave-ice interactions, and particularly the importance of wave fracture in determining the amount of lateral melt.

\subsubsection{Impacts on sea ice concentration over the satellite era}

We next analyse transient historical simulations covering the satellite era. We are unable to determine which of FSD-v1 and FSD-v2 is closer to real floe sizes, so here we consider the impacts on sea ice concentration from both. Fig. 6.4(a,c) show the monthly sea ice area cycle in both hemispheres, averaged over 1983-2014. There is no discernible difference between STD and FSD-v2. FSD-v1 accelerates the yearly melt for both hemispheres, moving the annual minimum forward from September to August, but the impact is small. Fig. 6.4(b,d) show the time series of sea ice area in August in the Northern 
Hemisphere and February in the Southern Hemisphere, when the impact of including FSD physics is greatest. Sea ice area from FSD-v1 during these months is consistently lower than the standard model (on average by 0.27 million $\mathrm{km}^{2}$ in $\mathrm{NH}$ August and 0.39 million $\mathrm{km}^{2}$ in SH February). In the Arctic, FSD-v2 has, if anything, very slightly higher sea ice area than the standard model. In the Antarctic, FSD-v2 sea ice areas are slightly lower than the standard model. There is a slight reduction in the magnitude of the Arctic trend in FSD-v1. This could be because there is less sea ice area at the beginning of the simulation than the standard model, meaning that there is less sea ice area to be lost as the climate changes. In February in the Antarctic, there are no sea ice area trends in any simulation.

(a) $\mathrm{NH}$

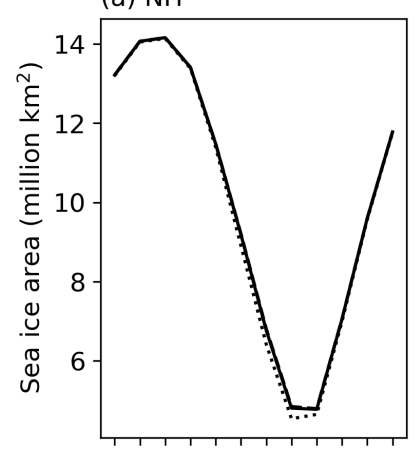

(c) $\mathrm{SH}$

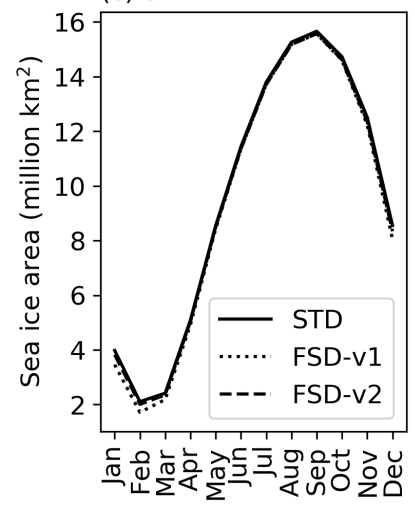

(b) NH Aug

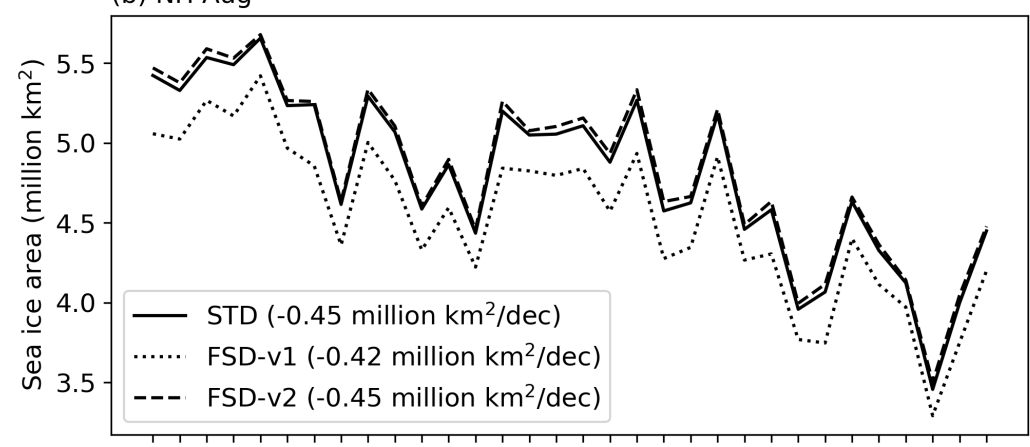

(d) SH Feb

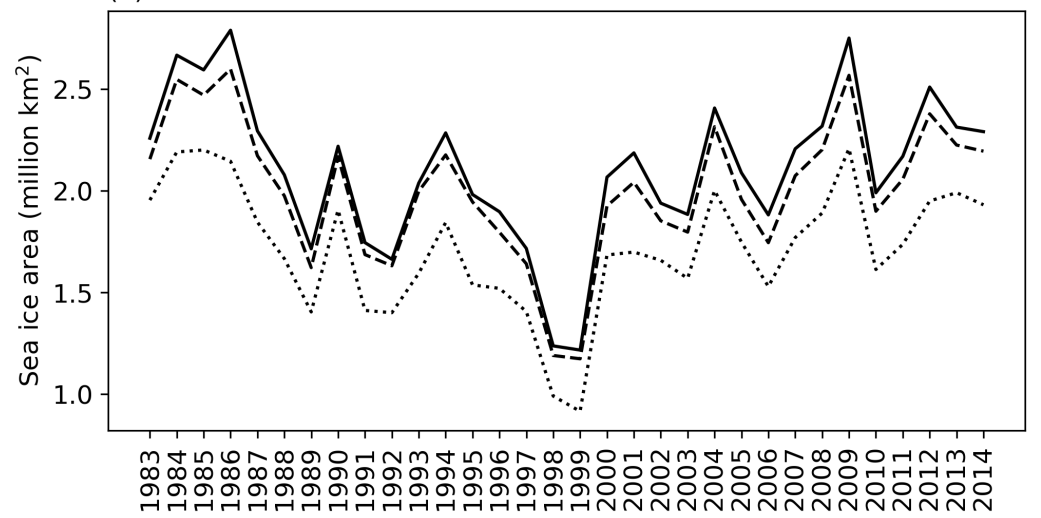

Figure 6.4: (a,c) monthly sea ice area in (a) the NH and (c) the SH averaged over 1983-2014. (b,d) sea ice area time series for (b) NH August and (d) SH February. Trends calculated from a least-squares regression are noted in the legend if they are statistically significant $(p<5 \%)$.

The impacts on sea ice area can be explained by considering the total amounts of lateral and basal melt (Fig. 6.5). With smaller floe sizes, FSD-v1 increases the amount of lateral melt by a factor of five relative to the standard model without a FSD. The increase in lateral melt is mostly compensated by a reduction in basal melt, although there is a 

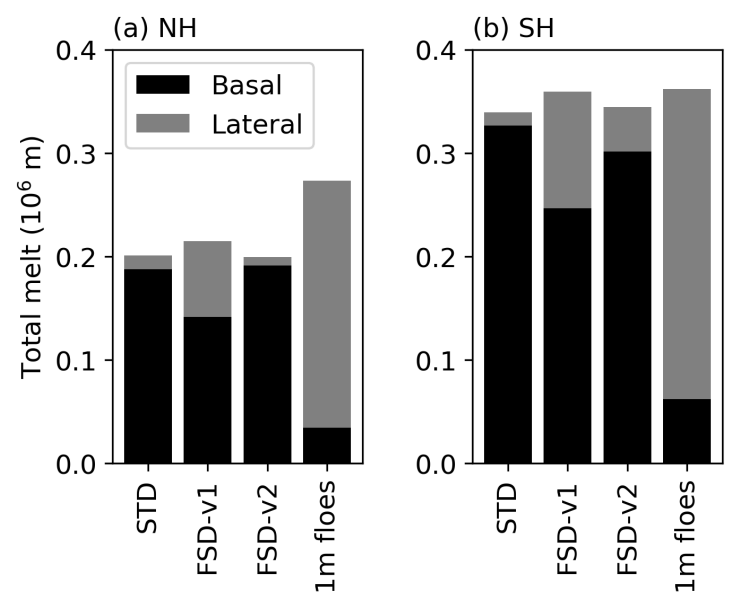

Figure 6.5: The total lateral (grey) and basal (black) melt from all months 1983-2014 for different simulations for (a) the Northern Hemisphere and (b) the Southern Hemisphere. ' $1 \mathrm{~m}$ floes' is the standard model simulation using a fixed floe size of $1 \mathrm{~m}$ that was presented in Chapter 3

slight increase in the combined total melt. For FSD-v2 in the NH, with many floes larger than $300 \mathrm{~m}$, the amount of lateral melt is slightly decreased relative to the standard model, compensated by a slight increase in basal melt. In the SH, FSD-v2 floes are smaller than the $\mathrm{NH}$, and so the amount of lateral melt is still larger than the standard model.

Chapter 3 found that CMIP5 models tended to simulate too much loose, low-concentration sea ice cover throughout the year, and too little compact, high-concentration cover in the summer, as a fraction of total sea ice extent. We speculated that these biases in the normalized sea ice concentration distribution could be partially explained by lateral melt: reducing floe sizes near the ice edge could remove spurious low-concentration ice, while increasing floe sizes could increase concentration near multi-year ice by repressing melt. Examining the normalized concentration distribution from our transient simulations (Fig. 6.6), we find that the changes in FSD physics make very little difference. The p-value calculated from a Kolmogorov-Smirnov test, which represents the confidence that the two populations come from the same distribution, is significant only in the SH between October and January when comparing STD to FSD-v1, and is a degradation relative to observations. Chapter 3 showed a reduction in the fraction of low-concentration sea ice in February when the constant floe size was reduced to an extreme value of $1 \mathrm{~m}$. This extreme floe size strongly enhanced lateral melt, with much smaller impacts obtained when floe sizes are determined by physical processes and therefore more realistic (Fig. 6.5). Thus, the floe-size-dependence of lateral melt does not explain the over-estimation of low-concentration cover in NEMO-CICE. 
(a) $\mathrm{NH} 10-20 \%$

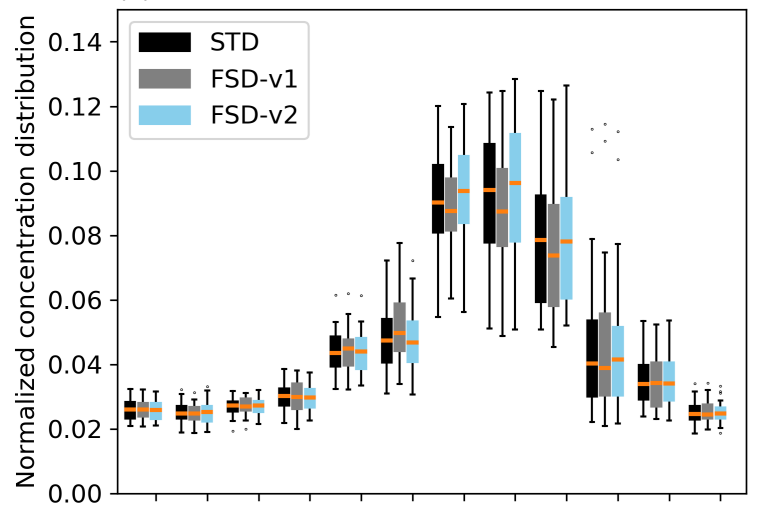

(c) $\mathrm{NH} 90-100 \%$

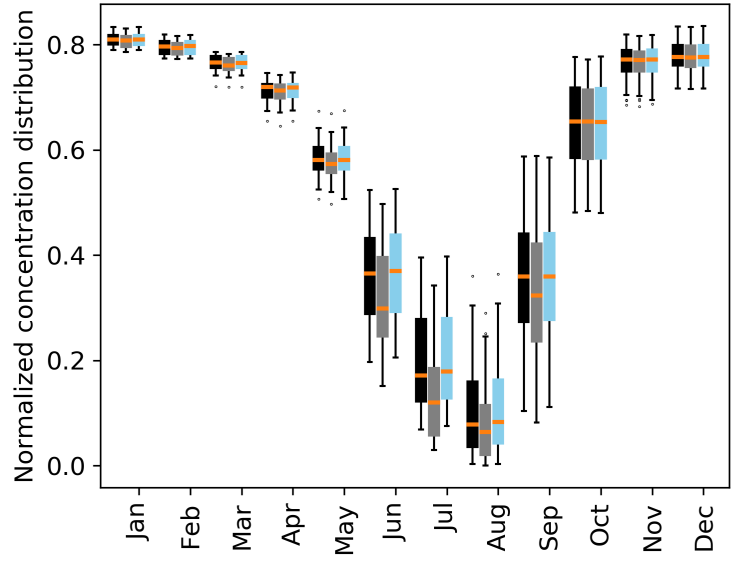

(b) $\mathrm{SH} 10-20 \%$

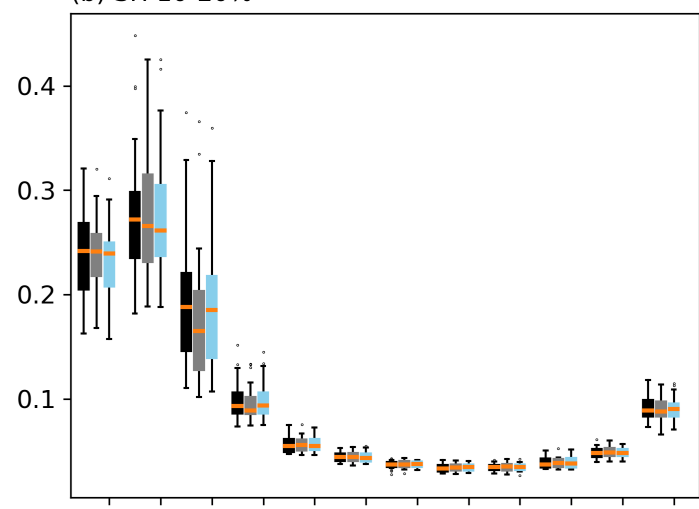

(d) $\mathrm{SH} 90-100 \%$

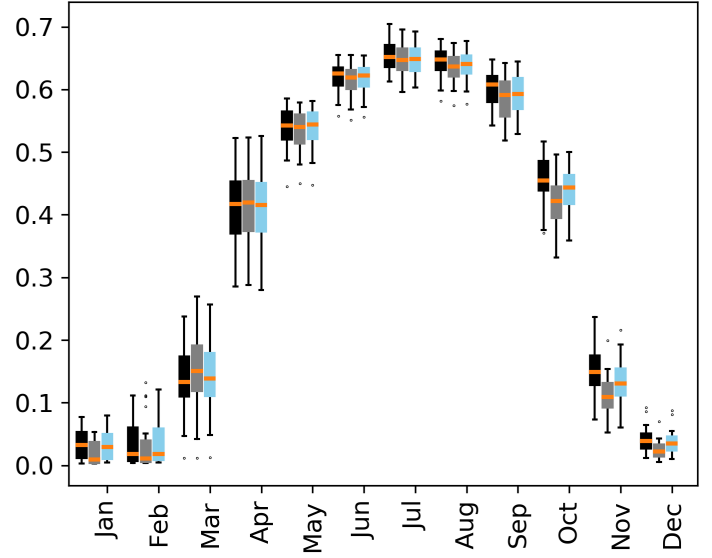

Figure 6.6: The 10-20\% bin from the normalized sea ice concentration distribution for each month, where boxes contain all years from 1983 to 2014, from three transient simulations in (a) the Northern Hemisphere and (b) the Southern Hemisphere. (c,d) as (a,b) but for the $90-100 \%$ bin. Boxes extend from the lower to upper quartile values of the data with a line at the median. Whiskers show 1.5 of the interquartile range; beyond this data are considered outliers and plotted as individual points.

The total amount of lateral melt differs substantially between our two versions of the FSD model, due their differing levels of fragmentation. The simulated floe perimeters must be validated with observations to determine the true impact of the FSD on lateral melt, and may differ if ocean surface waves were correctly included, rather than being drawn in using a simple scattering parametrization. In simulations where the amount of lateral melt increases, we find that there is a compensating reduction in basal melt, resulting in little change to the total amount of melt. This effect may differ across ocean models, particularly for different ocean model vertical resolutions and vertical mixing schemes, which would alter the heat flux available for sea ice melt in the top ocean model grid cell. Furthermore, use of a dynamical atmosphere model would permit atmospheric 
feedbacks in response to enhanced lateral melt and reduced surface albedo that may alter results. While the impact of floe-size-dependent lateral melt is small in the coupled sea ice-ocean simulations considered here, further work is required to determine its relevance for the polar climate system.

Using the model physics considered here, the floe-size-dependence of lateral melt may play a larger role at shorter timescales rather than longer timescales, for example rapid fracture events during storms, with implications for near-term sea ice forecasting. We only consider monthly model output in this thesis. Extreme events are not wellrepresented with our current model framework: use of a look-up table constrains the amount of fracture that can occur, six-hourly wave forcing is likely too coarse temporal resolution to capture storms, and lack of wind input to waves may not well-represent the intensity of large wave events. The role of FSD physics in determining sea ice response to storms on seasonal and sub-seasonal timescales would be an interesting question for future work.

\subsubsection{Impacts of Arctic climate change on the FSD}

Our experimental set-up of a free-running sea ice model including a FSD forced by an atmospheric renalysis and wave hindcast is well-placed to investigate how changes in climate in recent decades have affected sea ice fragmentation. Given the higher physical fidelity of the wave-dependent new ice growth scheme, we use the simulation FSD-v2, which was run over 1975-2014 with the period 1983-2014 used for analysis (see Appendix B). We consider the Northern Hemisphere only in this Subsection, as Arctic sea ice has exhibited more marked change over this period than Antarctic sea ice. Simulated Arctic sea ice area decreases year-round, most strongly in September. September sea ice declines with a linear trend of $-13.5 \%$ decade (Fig. 6.7a), in good agreement with satellite observations from Fetterer et al. (2017).

As trends in the total perimeter will be heavily skewed by the strong decline in sea ice area, we show the trend in perimeter per sea ice area, $P_{i}$, in Fig. 6.7c. $P_{i}$ is calculated by integrating $P_{o}$ over grid cell areas, and then dividing by the total sea ice area, with trends obtained from a least-squares linear regression. In the months where trends are statistically significant $\left(p<5 \%\right.$ ), $P_{i}$ increases by 5.5 to $14.8 \%$ per decade, with a maximum in October (Fig. 6.7c). Sea ice floe sizes are decreasing, or the ice cover 

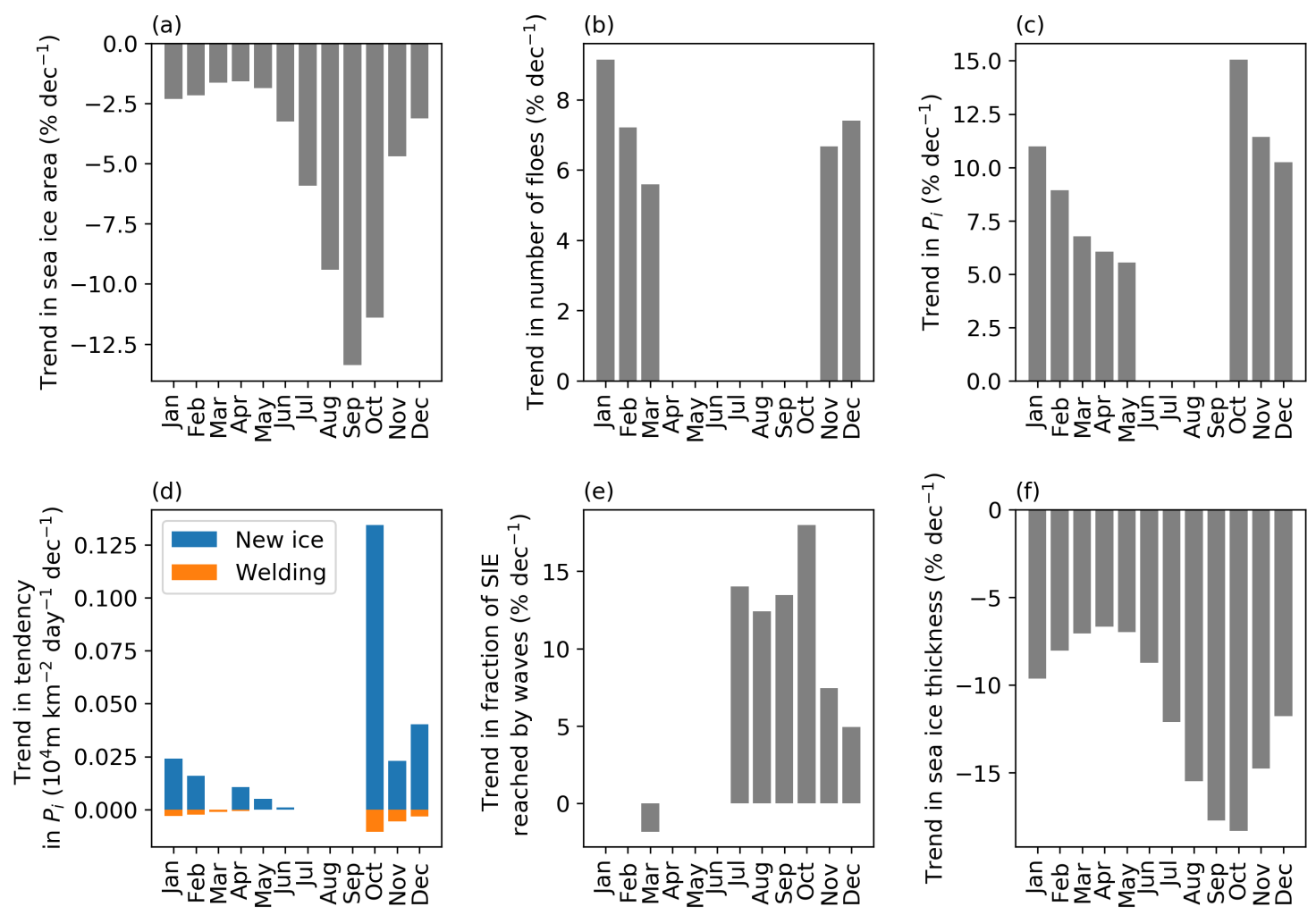

Figure 6.7: Northern Hemisphere trends over 1983-2014 relative to the 1983-2014 mean calculated from a least-squares linear regression (where statistically significant, $p<5 \%$ ) in (a) sea ice area; (b) the total number of floes; (c) total perimeter divided by sea ice area, $P_{i}$; (d) the average contribution to $P_{i}$ arising from FSD-dependent processes that have statistically significant trends; (e) the fraction of sea ice extent where ocean surface waves are active; and (f) average sea ice thickness.

is becoming more fragmented, as the climate changes. Of the five FSD-dependent processes, two show statistically significant trends in their impact on $P_{i}$ over this period: wave-dependent new ice growth and floe welding (Fig. 6.7d). Floes become less likely to weld together as concentrations decrease. The contribution to $P_{i}$ from new ice growth increases over 1983-2014, most strongly in October, meaning that initial floe sizes are increasingly smaller and pointing to increasing wave activity.

To investigate this further, we calculate the average significant wave height within the ice and outside the ice in the Northern Hemisphere. The only statistically significant monthly trends in significant wave height outside the ice are downwards ( -1.9 and $-1.4 \%$ per decade in August and November respectively). Within the ice, significant wave height increases by $14 \%$ per decade in October, with no trends in other months. The fraction of sea ice that waves access in the Northern Hemisphere trends upwards by $5-18 \%$ per decade from July to December with a peak in October (Fig. 6.7e). This suggests reduced 
wave attenuation, i.e. that waves travel further into the ice. In our model, attenuation depends on wave period, sea ice thickness and the number of floes encountered. The total number of floes in the Arctic is increasing (Fig. 6.7b), which would increase attenuation, and there is no significant trend in wave period outside the ice (not shown). Meanwhile, average sea ice thickness has large downward trends in all months, with a maximum of $18 \%$ per decade in October (Fig. 6.7f). This suggests that reduced attenuation is due to the thinning of the ice cover in our model.

This analysis suggests that ocean surface wave interactions have become increasingly important for the Arctic sea ice zone in recent decades. Our results would be strengthened if other attenuation schemes (e.g. Bennetts and Squire 2012; Meylan et al. 2014) showed similar trends when forced by changing Arctic atmospheric and wave conditions. When coupled to an ocean surface wave model and following model validation, simulations

such as these could provide useful forecasts for polar operations. As the thinning of Arctic sea ice is projected to continue (Holland et al., 2006; Rampal et al., 2011), our results point to further penetration of the sea ice cover by ocean surface waves in the future. Besides the relevance to Arctic stakeholders, increasing access of ocean surface waves to the high latitudes would change the characteristics of the sea ice cover as well as having implications for near-surface atmosphere and ocean interactions.

\subsection{Conclusions}

In conclusion, we find that the addition of a wave-dependent growth scheme causes large changes in simulated floe sizes and moderate changes in the impact of different physical processes on the number FSD. It enhances the role of ocean surface waves, as there are more large floes to break, and reduces the role of floe welding, as there are fewer small floes to weld. The changes obtained with the addition of a wave-dependent new ice growth scheme (FSD-v2 vs FSD-v1) are larger than the changes obtained by extending floe size categories (Appendix C), suggesting that process uncertainty is more important than uncertainty due to floe size category truncation at this stage of model development.

We propose use of the floe perimeter as a metric for model assessment due to its role in determining the concentration change arising from lateral melt. The simulated seasonal cycle in floe perimeter suggests an asymmetry that could be tested with further observa- 
tions. Examining how different FSD processes contribute to floe perimeter, we find that new ice growth and wave fracture are the most significant. During the months when lateral melt occurs, wave fracture is the dominant FSD process driving floe perimeter, making uncertainty in the representation of sea ice fracture by ocean surface waves particularly significant for simulated lateral melt.

We show that the impacts of FSD-dependent lateral melt on sea ice area in historical coupled sea ice-ocean simulations of our model are small and impacts on the concentration distribution are essentially zero. In contrast to the extreme parameter perturbation experiment with a constant floe diameter of $1 \mathrm{~m}$ discussed in Chapter 3, there is no enhancement in lateral melt arising from addition of an evolving FSD including nilas growth in our current model configuration. However, we stress that the amount of lateral melt depends on the level of fragmentation in the model, which is as-yet unconstrained. We find that changes in lateral melt are mostly compensated by basal melt, resulting in very small changes in total melt for simulations with evolving FSDs. Further research is required to determine whether this compensation effect is realistic and is a robust response among different ocean models. Finally, we show that trends in wave-dependent new ice growth drive trends towards larger floe perimeter per sea ice area in the Arctic, representing a more fragmented sea ice cover. In our simulations, ocean surface waves are increasingly able to access ice-covered regions as a result of reduced wave attenuation, which occurs due to the thinning of Arctic sea ice. 


\section{Chapter 7}

\section{Discussion}

In this chapter, I draw together the work presented in Chapters 3-6 to answer the questions posed in Chapter 1 in the context of relevant literature.

\subsection{What are the processes that control the sea ice floe size distribution?}

The sea ice floe size distribution was first defined and measured in the early 1980s (Rothrock and Thorndike, 1984). Since then, few studies have quantitatively examined how different physical processes determine the spatial distribution and temporal evolution of floe size. Qualitatively, most studies have attributed FSD behaviour to wave fracture, neglecting the contributions of other processes. The FSD and its evolution have so far been poorly observed (Figs. 2.4 and 2.5) and concerns have been raised over the statistical analyses used to produce number density distributions (Stern et al., 2018b). Moreover, few observational studies have made their data publicly available.

Output from a geophysical model can provide data over much larger spatial and temporal domains than point observations. As an example, the record of sea ice thickness, which has only recently begun to be estimated from satellite, has been complemented by output from sea ice model hindcasts (Schweiger et al., 2011; Massonnet et al., 2013). Perhaps more importantly, development of a geophysical model can provide an independent test of observed behaviour. However, developing a model based on assumptions made from observations and comparing it to the same observations is not an independent test. Contemporary modelling studies have based descriptions of wave fracture on observa- 
tions, assuming that fractured floe sizes are redistributed equally to all lower sizes (Zhang et al., 2015, 2016) or that wave fracture must result in a power law distribution (Williams et al., 2013a,b; Bennetts et al., 2017). These descriptions are diagnosed from observations and do not constitute an independent test of our understanding of observed behaviour. Descriptions of processes other than wave fracture have sometimes been somewhat arbitrary, for example doubling the floe diameter in a grid cell during freezing conditions (Bennetts et al., 2017).

In light of these considerations, I chose to adopt a process-based approach to model development in Chapter 4, describing the physics of how different processes act on floes and providing an independent test for our understanding of floe behaviour. In the future, such a model could be used to inform development of parametrizations for reducedcomplexity models of the FSD. We obtained insights on the processes controlling the FSD throughout model development and analysis. The initial planning of the model alone encouraged consideration of the sea ice system as a whole, as I sought to describe evolution of floe size throughout the ice pack and throughout the year. This required prioritization of processes considered to be of first-order importance for simulation of the FSD. It also highlighted gaps in existing literature, notably the almost complete lack of studies on floe freezing processes (Chapter 5).

In Chapter 4, parametrizations of processes for FSD evolution are based on underlying physics, which means that parameters in the model equations correspond to physicallyobservable quantities. We were aided in several processes by the descriptions presented in Horvat and Tziperman (2015), but these required adaptation for implementation in a large-scale model. The processes described in Horvat and Tziperman (2015) were not sufficient to describe the growth of floes in winter. I proposed a description of a new physical process, the welding together of floes during freezing conditions, that also has a physical basis and can be tested with observations, as shown in Chapter 5 .

A key outcome from this work is the comparison of the relative importance of different processes to overall simulated FSD behaviour, in space, time and across floe sizes. This is possible because our FSD emerges due to the interaction of different physical processes, rather than being tuned to reproduce certain behaviour (e.g. Zhang et al. 2016, Bennetts et al. 2017). The decomposition allows results to be compared to studies that 
have considered physical processes individually (e.g. Montiel and Squire 2017, Herman et al. 2018, Horvat and Tziperman 2017). It also provides motivation for further investigation of certain processes, using models and/or observations.

In Chapter 4, I demonstrated the dominant role of floe welding in setting floe sizes throughout the ice pack when sea ice initially grows at the smallest floe size. It is unique amongst the processes considered in Chapter 4 in having strong effects throughout the ice pack, not just in the marginal ice zone. Floe welding contributes to scale-invariant behaviour particularly as freeze-up begins. The importance and impact of this process had not previously been suggested. We confirmed the importance of wave fracture in the creation of small floes with radii below $100 \mathrm{~m}$. There is a greater likelihood for large floes to be fractured by waves compared to small floes, driving a slight change in slope in the number FSD on a log-log plot. In the Southern Hemisphere, floes are redistributed preferentially to certain lower sizes by wave fracture. This behaviour has some qualitative similarities to that observed by Herman et al. (2018) and simulated by Montiel and Squire (2017), and arises without prescription. Lateral melt is the dominant process for reduction of floe sizes. This is in contrast to lateral growth, which has an impact several orders of magnitude lower.

Further insight into the roles of different processes in controlling the FSD was obtained from the observations presented in Chapter 5. I stress that these results represent only a single point in the Arctic, but as the first quantification of floe freezing processes in-situ they contain some useful insights. The observations showed that our description of floe area growth by welding was reasonable and provided an order-of-magnitude estimate of the floe welding constant, which was close to our initial guess based on model performance, before observations were analysed. They also indicated that lateral growth was more dominant for small floes than suggested by the model, but there are limitations in this comparison. A key result from Chapter 5 was evidence for the large impact of waves in determining initial floe sizes, which were well-described by the model of Shen et al. (2001). These results translated easily to a new model parametrization for the initial sizes of floes described in Chapter 6.

Including the impact of waves on initial floe size in our updated model had some consequences for process contributions. More large floes are available to be fractured, 
and fewer very small floes are available to be welded together. The average tendency in the number FSD arising from new ice growth shows some power-law-like characteristics. This is another process that could partially explain observed FSD behaviour, but has not been suggested by previous studies. I also found that both the addition of a wave-dependent scheme for new floe sizes and an extension of floe size categories alter the impact of wave fracture on the floe number distribution, by smoothing wave fracture redistribution to sizes below $100 \mathrm{~m}$ and making the discontinuity at around $100 \mathrm{~m}$ more apparent. This behaviour is in qualitative agreement with observations of a wavedetermined transition in FSD slope (Toyota et al., 2006; Steer et al., 2008; Toyota et al., 2011, 2016; Geise et al., 2017) and agrees less with the findings of Herman et al. (2018) and Montiel and Squire (2017) than our initial model.

While in Chapter 4 I showed process contributions to the number FSD for different floe sizes and to the representative radius in space and time, in Chapter 6 I proposed use of the total ice perimeter. This metric is more relevant for climate modelling purposes than the number FSD as it describes the susceptibility of the ice pack to lateral melt. It also condenses information on the FSD to a single number that can be shown in time and space, without requiring assumptions as the shape of the FSD. Tendencies in total perimeter arising from different physical processes highlight the role of wave-ice interactions in determining lateral melt. Perimeter is most strongly determined by wave-dependent ice growth throughout most of the year, except during the summer months when lateral melt occurs, when it is most strongly determined by wave fracture. These results motivate further observational studies to develop our understanding of how ocean surface waves break up ice and limit floe growth.

\subsection{What is the relationship between sea ice floe size and the polar climate system?}

As has been previously discussed in the literature, the size of sea ice floes determines the impact of lateral melt (e.g. Steele 1992), with a given area of small floes resulting in reduced sea ice concentration relative to the same area having fewer but larger floes. Chapter 3 provided evidence from CMIP5 that inclusion of lateral melt in models improves consistency with observations for the sea ice concentration distribution. This chapter also 
demonstrated that assuming a fixed floe diameter of $1 \mathrm{~m}$ in the standard version of the coupled sea ice-ocean model NEMO-CICE significantly reduced sea ice concentrations by enhancing lateral melt.

In coupled sea ice-ocean simulations incorporating a FSD, the average impact on summer Antarctic sea ice area over the satellite era is around 0.4 million $\mathrm{km}^{2}$ for the first model version and 0.1 million $\mathrm{km}^{2}$ for the second. For comparison, the difference in the Antarctic sea ice area summer minimum between two standard climate models using CICE that differ only in their representation of atmospheric physics (ACCESS1-0 and ACCESS1-3, Fig. 3.1) is close to 3 million $\mathrm{km}^{2}$. This suggests a greater role for the atmosphere than for sea ice model physics in the underestimation of the Antarctic summer minimum in many CMIP5 models. The minimal impact of the second FSD model version, which has greater physical fidelity, suggests it is unlikely that the direct effect of enhanced lateral melt arising from an evolving FSD is of first-order importance for the climate system in the absence of atmospheric feedbacks. Thus, while lateral melt is important for simulation of sea ice concentration, representing its average effect through simple parametrizations may be sufficient for long-term climate simulations.

However, to conclude that the impact of floe-size-dependent lateral melt on the polar climate system is minimal requires confidence that the model represents both floe sizes and the response to lateral melt correctly. I stress that the modelled floe sizes have not been validated by observations and may differ if other FSD processes and a more comprehensive scheme for ocean surface waves are included. In simulations with small floes, a large amount of lateral melt occurs, but is mostly compensated by a reduction in basal melt. It is not known if this response is realistic or dependent on the ocean model used. Furthermore, all simulations presented here used a fixed atmosphere, preventing atmospheric feedbacks that may occur in response to enhanced lateral melt. Further work is required to determine the true impact of floe-size-dependent lateral melt on the polar climate system.

On a regional scale, I expect enhanced lateral melt to be important, particularly where ice is heavily fragmented. Wave fracture is the dominant process to increase sea ice perimeter during the summer melt season (Chapter 6), and it tends to occur in discrete storm events (Perovich and Jones, 2014), which are poorly represented by our model set- 
up using a wave hindcast (Subsec. 6.3.3). Enhanced lateral melt may therefore be more important on shorter timescales, for example by accelerating ice retreat during storms, than on climate timescales. Our model could easily be coupled to a wave model and run at high-resolution over a short time period to investigate this further.

Our evolving floe size model forced by a reanalysis atmosphere and waves can be used to examine how changes in the polar climate system affect sea ice floe size. As the climate warms and Arctic sea ice thins, our model simulates both higher wave heights inside the sea ice region and a greater area of sea ice where waves penetrate in the Northern Hemisphere, reducing floe sizes (Chapter 6). A coupled wave-ice model could be used to examine the interactions between thinning sea ice and a possible increase in ocean surface wave activity. Changes in wave activity in the sea ice zone may alter atmospheric boundary layer processes and turbulent mixing.

Although I have focused on lateral melt in this thesis, a number of other floe-sizerelated processes may impact climate. The FSD simulated by our model could be used to determine choice of sea ice rheology, the likelihood of floe collisions, and the surface form drag. Changes to the representation of these sea ice model components may alter sea ice dynamics and near-surface atmospheric and oceanic heat fluxes. The FSD may be relevant for the generation of ocean eddies at floe edges due to melting and freezing around floes (Smith, 2002; Horvat et al., 2016). Of particular importance to climate is the distribution of sea ice leads, which allow vast amounts of turbulent heat transfer between the atmosphere and ocean (Marcq and Weiss, 2012). Current sea ice models assume that the open water fraction is uniformly distributed throughout each grid cell. The simulated FSD could be used to infer a probability distribution of lead widths; smaller floes and narrower leads would allow greater heat loss (Worby and Allison, 1991). Our model could provide a starting point from which to investigate the importance of these other processes.

\subsection{How do we integrate observations and models to bet- ter understand sea ice?}

Both climate models and observations are essential to understand the physics of the highly-complex sea ice system. In practice, there is often a disconnect between the mod- 
elling and observational communities. This can result in a significant lag between observational and modelling studies of the same phenomena that may limit our understanding of past, present, and future sea ice cover.

There are a number of ways that geophysical models can inform observations. This can be illustrated with examples from the present study. Firstly, trying to describe evolution of climate variables in space and time for model development highlights observational gaps. In this study, during development of the FSD model, I identified floe freezing processes as being particularly poorly-observed, motivating Chapter 5. Secondly, comparing the relative importance of simulated processes highlights those which are most important to constrain. Note that this comparison can only be made in a process-based model. In our case, floe welding has large impacts-meriting more observational attention than has previously been paid-while lateral growth has much smaller impact-and is therefore less important to observe. Similarly, sensitivity studies to different model parameter values indicate which parameters are most important to constrain. Chapter 4 shows that simulation results depend strongly on the floe welding parameter. Given the large process uncertainty in the FSD model (Chapter 6) however, process uncertainty should perhaps be prioritized ahead of parameter value uncertainty. Finally, model simulations may show relationships or behaviour that provide a hypothesis to be tested with observations. For example, in Chapter 6, I showed that the simulated seasonal cycle in the total number of floes in the Northern Hemisphere is asymmetric, being slower to grow in winter than melt in summer. This could motivate an expansion of the observational work presented in Perovich and Jones (2014) to examine whether this behaviour occurs in reality.

The present study also provides examples of how observations can be used to inform models. In the first instance, observations may help to identify new physical processes and suggest functional dependencies for process descriptions. The author observed the freezing together of floes during a research voyage to Antarctica, motivating further theoretical work and inclusion of this process in the FSD model. Observations can be used to test the physical descriptions of individual processes. This approach formed the basis for Chapter 5, where the sizes of floes were compared to that predicted by individual process models for lateral growth, floe welding, and pancake ice formation, giving useful insights for model development. Similarly, observations may provide estimates for parameter val- 
ues, providing that models include functional dependencies that agree with observations and parameters that correspond to physical observables. A contrasting example that appears in some models is the critical ice thickness of $4 \mathrm{~m}$ beyond which it is assumed that advection ceases (Bryan, 1969; Gordon et al., 2000), a threshold which does not exist in the real world. The tensile stress mode parameter and a lower bound for the floe welding coefficient were computed from observations in Chapter 5. Note that model parameter values approximate variables that may vary in space and time by a single number and so should ideally be measured at multiple space and time points.

These model-observation comparisons are made at the process level. The overall results of the interacting processes in a climate model should also be compared to observations, for example comparing area-integrated FSDs or using the integrated ice area error (proposed in Chapter 3). With climate models as opposed to individual process models, other modelling uncertainties must be taken into account during comparisons. When coupled to ocean and atmosphere models, intrinsic internal variability can account for significant differences between models and observations (Sec. 2.4). Climate models may differ substantially in their representation of the physics of the climate system. Insights for model development can be gained if all models show similar biases-indicating poor process representation common to all models-or groups of models clustered by process descriptions show the same behaviour. The Coupled Model Intercomparison Project (CMIP) archive provides a wealth of information used in Chapter 3 to identify consistent biases in the distribution of sea ice concentration relative to observations. Grouping models by their representation of lateral melt indicated that inclusion of this process partly resolved the bias, as did parameter perturbations in the existing lateral melt scheme in a coupled ocean-sea ice model. Comparisons to satellite sea ice observations must be made carefully due to observational uncertainty: the algorithms which convert passive microwave brightness temperatures to gridded sea ice concentrations, like models, require assumptions, and struggle with atmospheric effects and low concentrations and thicknesses.

Despite observational uncertainty, satellite sea ice concentration observations are highly useful for model validation due to their global and relatively long temporal coverage, compared to other sea ice observations, spanning 1978 to the present day. In contrast, FSD observations are sparse (Figs. 2.4 and 2.5). FSD models are in the very early stages of 
development, so exact sizes or distribution properties are unlikely to match up with observations. However there may be general characteristics that models capture, such as the location of a transition in floe size, the depth of wave penetration or the seasonal evolution of the number of floes. Use of existing FSD data sets would require model experiments forced by reanalysis at high spatial resolution over the time period where observations are available. It would also require access to the data sets, as the derived results presented in the papers often focus on power law exponents that should be reanalysed (Stern et al., $2018 b$ ). It is anticipated that progress will be made by exploitation of synthetic aperture radar imagery to calculate the depth of wave penetration (Shen et al., 2018) and of satellite altimeter products to calculate floe sizes as their spatial resolution increases. Another candidate for model validation is microwave backscatter data, which can be processed to generate surface roughness, a variable that may suggest a transition from small to large floes or a marginal ice zone.

These comparisons could provide insight on where FSD models are deficient. A more direct way for observations to inform models is to consider the inverse problem of optimization (e.g. Tett et al. 2013; Roach et al. 2017). Here, a cost function describing the difference between some chosen simulated and observed variables is minimized by varying multiple model parameter values within physically reasonable bounds. Such an approach could be used for the FSD model if suitable observations with low uncertainty become available.

Thus, insights from observations can inform modellers throughout the model development process: suggesting new variables that should be prognostically simulated (such as the FSD), prioritizing processes to be included in the model, refining descriptions of processes, constraining parameter values and evaluating the model. Conversely, development of a model for a new variable can motivate more observations of that variable and of the processes that determine it, if model simulations deem them to be of importance. 


\section{Chapter 8}

\section{Conclusions}

Climate model projections of future change are essential tools for policy-makers in the current era of rapid global warming. Confidence in projections for the future requires accurate simulation of different climate variables in the past. However, simulation of Antarctic sea ice extent over the recent historical period in global climate models does not compare well to observations (Turner et al., 2013; Zunz et al., 2013). In Chapter 3, we evaluated Antarctic sea ice as simulated by climate models using a more comprehensive set of metrics than these previous studies, taking into account sea ice concentration values and the regional distribution of sea ice. We found that current climate models struggle to simulate the heterogeneity of sea ice, with biases common to many models and the high and low ends of the sea ice concentration distribution.

That models with diverse physics exhibit the same biases suggests a deficient representation of physical processes common to many models. In Chapter 3, we found that inclusion of lateral melt - the melting of sea ice floes at their edges - could partially explain the biases in the climate models considered. We also found an improvement in the sea ice concentration distribution when artificially enhancing lateral melt through changes to the constant floe size parameter, which determines the total area of floes exposed to the ocean. This, together with other recent studies alluding the importance of sea ice floe size and the lateral melt feedback (Perovich et al., 2008; Kohout et al., 2014; Asplin et al., 2014), motivated development of a model for prognostic simulation of FSD, with the aim of improving simulation of sea ice relative to observations.

Development of the FSD model, presented in Chapter 4, formed the major part of this 
thesis and was a significant technical and mathematical challenge. While guided by the theory presented in Horvat and Tziperman (2015), adaptation into a climate-scale model required additional work to ensure compatibility with the existing sea ice model and to strengthen the description of floe freezing processes. A major guiding aim was that our model would be based on robust physical principles. The key achievement of this chapter was simulation of a FSD in a global sea ice-ocean model showing characteristics in line with observations, that arose without initialization from the interaction of different physical processes. This unique model provides a robust framework which can easily be improved by the addition of new physical processes in the future.

Driven by the aim to develop a model grounded in underlying physics, we considered how observations could inform new physical parametrizations. In Chapter 5, we conducted an observational study, quantifying floe freezing processes in-situ for the first time to aid model development. This validated our physical descriptions of individual processes, provided constraints on model parameters, and suggested addition of a new physical description for how the ocean surface wave field limits the sizes of new ice floes.

In Chapter 6, we updated the FSD model to include wave-limited ice growth, increasing the physical fidelity of the model. This change increased the sizes of modelled floes, resulting in a very small overall change in the amount of lateral melt compared to the simulation without floes. Further work is required to determine whether both the modelled floe sizes and the climate model response are both realistic and robust across different coupled models. However, with our current model configuration and physics, our results suggest that the floe-size-dependence of lateral melt is not of first-order importance for global model simulations on climate timescales.

Although this was initially the primary motivation for constructing a model to prognostically simulate the FSD, high interest in the development of FSD models from the sea ice scientific community suggests that they are useful for other reasons. Simulation of FSD is necessary to include wave-ice interactions, non-continuum rheologies and form drag in sea ice models. It may also be relevant for representing the spatial distribution of leads and sub-grid-scale ocean eddy effects, and for ecological modelling. Moreover, floe size itself, which can be used to describe ice fragmentation, is useful variable to predict for polar operations and Arctic communities. We have made significant advances towards 
predictive capability for FSD in this thesis, including quantification of how different physical processes control the FSD, and providing evidence of spatial and seasonal variability that could be tested with observations.

Future work could include:

- Development of two-way coupling between a sea ice and an ocean surface wave model

- Investigation of feedbacks related to storms on short timescales in a coupled atmosphereocean-sea ice-waves model

- Investigation of the lateral melt feedback on longer timescales in a coupled atmosphereocean-sea ice model

- Development of satellite products on a sufficient spatial and temporal scale for estimation of floe size to be used for model validation

- Further refinement of descriptions for physical processes governing the FSD and addition of new ones, such as mechanical redistribution

- More detailed analysis of how different physical processes may lead to power law emergence on different spatial scales and in different regions

- Investigation of how modelled changes in floe perimeter may be relevant for shipping routes

The research presented in this thesis has provided new insights into the behaviour of sea ice and opens up many opportunities in sea ice and polar climate modelling. 


\section{Appendix A}

\section{Sensitivity to floe welding parameter}
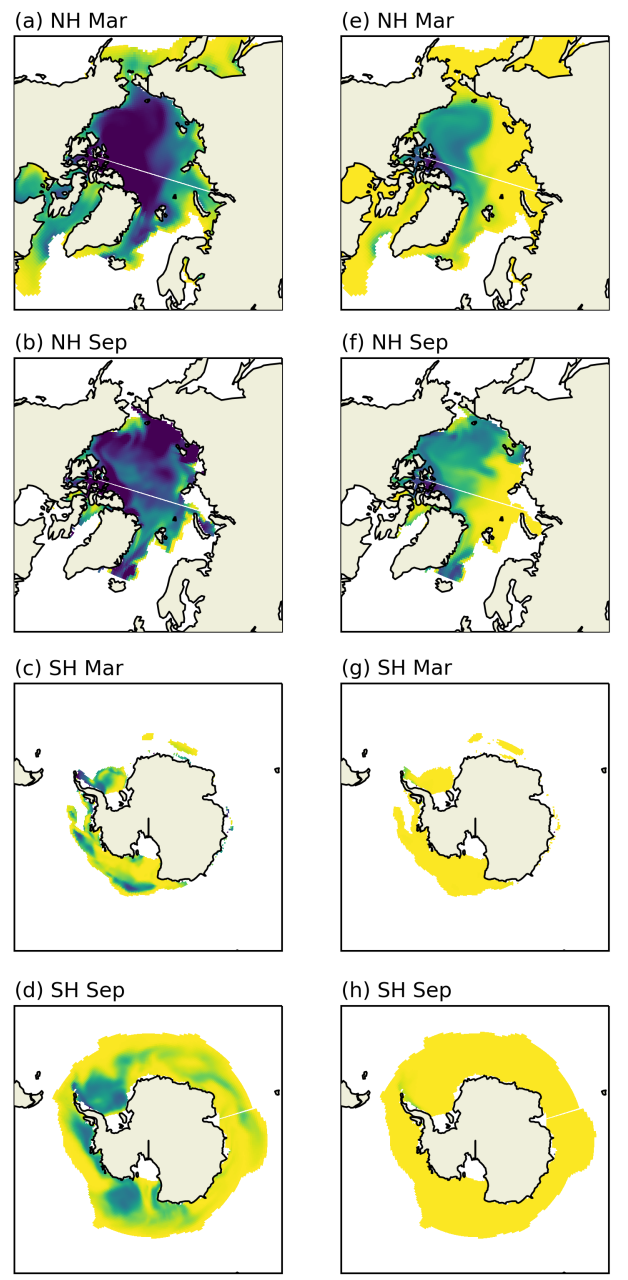

Representative radius $(\mathrm{m})$

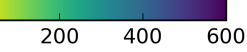

Figure A.1: The simulated representative floe radius, averaged over twenty years following spin-up, in (a, c) March and (b, d) September using a floe welding parameter of $\kappa=0.01 \mathrm{~m}^{-2} \mathrm{~s}^{-1}$. (e-h) as (a-d) but with a floe welding parameter of $\kappa=0.001 \mathrm{~m}^{-2} \mathrm{~s}^{-1}$. The range displayed is chosen to display both hemispheres on the same scale; dark purple may be greater than $600 \mathrm{~m}$. 


\section{Appendix B}

\section{Model adjustment for transient simulations}

The transient historical simulations conducted for this chapter were initialized in 1975 . There is a discontinuity in the JRA-55 reanalysis at year 1979 (Fig. B.1a), when satellite data becomes available for assimilation. Global average $10 \mathrm{~m}$ air temperature warms by over a degree at the annual minimum and a smaller amount at the annual maximum. Following Chapter 4, we consider time series of sea ice volume at the annual minimum to evaluate the time period required for the model to adjust to this forcing change. Northern Hemisphere $(\mathrm{NH})$ sea ice volume drops abruptly within two years of the change (Fig. B.1b). The amplitude of the Southern Hemisphere ( $\mathrm{SH}$ ) sea ice area annual cycle shrinks (Fig. B.1c), with the annual minimum in sea ice volume slowly increasing from 1979 to appear more stable from 1983 onwards (Fig. B.1b). That the SH summer minimum sea ice area and volume increase in response to an increase in air temperature is not self-evident. It is possible that this is a negative feedback whereby reduced winter sea ice provides less insulation between the cold atmosphere and relatively warm ocean, resulting in less sea ice melt in the summer. Another possibility is that a change in SH circulation occurs with the introduction of satellite observations. For the purposes of presenting results in Chapter 6, we use the period from 1983-2014 for analysis. 

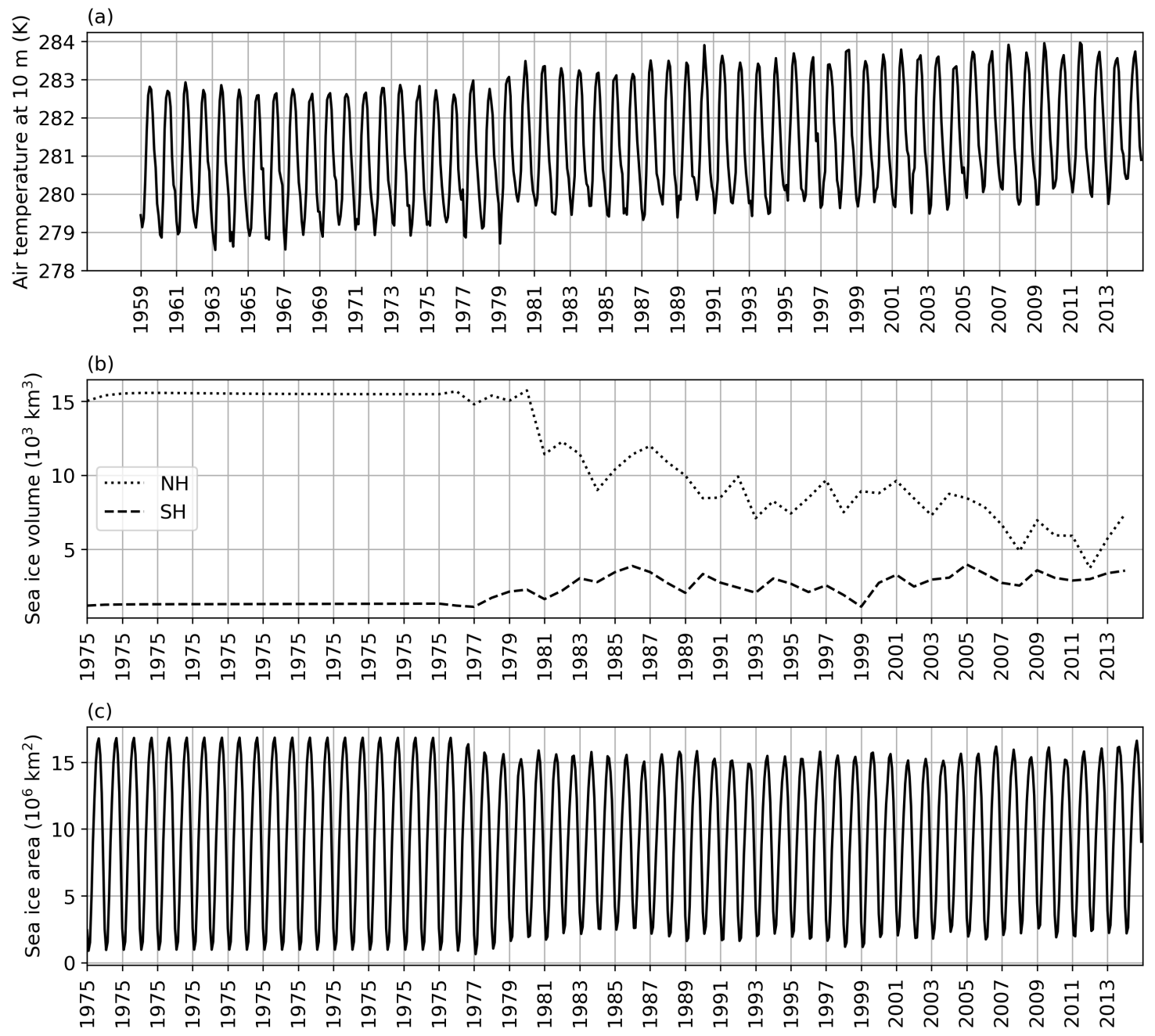

Figure B.1: Time series of (a) global average air temperature at $10 \mathrm{~m}$ from the JRA-55 reanalysis over 1958-2014; (b) sea ice volume at the annual minimum in the Northern and Southern Hemispheres from the FSD-v2 simulation (for twenty climatological years followed by transient forcing for 1975-2014); and (c) sea ice area in the Southern Hemisphere from the FSD-v2 simulation. 


\section{Appendix C}

\section{Sensitivity to floe size categories}

Some maximum floe size must be defined in our model of the sea ice FSD, as the categories cannot stretch to infinity. To investigate whether choice of maximum floe size alters the impacts of different physical processes, we present here an additional simulation where floe size categories are extended. We add a further 12 categories to the original 12 categories, with the additional category boundaries extending along the same exponential distribution to a maximum radius of $40 \mathrm{~km}$ (Fig. C.1). We include the wave-limited ice growth scheme in a twenty-year climatological simulation with these extended floe size categories, and use the second ten years for analysis.

With the increase in floe size categories, the hemispheric number distributions show

a decrease with increasing floe size until the last floe size category in both March and September for both hemispheres (Fig. C.2a-d). It appears that the truncation of floe size categories causes an accumulation of floes in the largest category only, rather than the last two categories as occurs in several months in the FSD-v1 and FSD-v2 simulations using 12 floe size categories (Figs. 4.2, 6.2). Fitting a straight line in log-log space to the number FSD for all floe size categories but the last, the slope is flatter (slope varies between -3.8 and -4$)$ compared to FSD-v1 ( -4.5 to -4.7$)$ and FSD-v2 with twelve floe size categories ( -4.2 to -4.4$)$.

In this simulation with extended floe size categories, the accumulation of floes in the largest category is driven by nilas growth only (Fig. C.2e-h). As discussed above, this behaviour in the model arises in grid cells with open water and no wave information, where new floes are set to the size of the largest category available. The addition of an ocean surface wave model may serve to reduce this accumulation, as in reality, while wave am- 


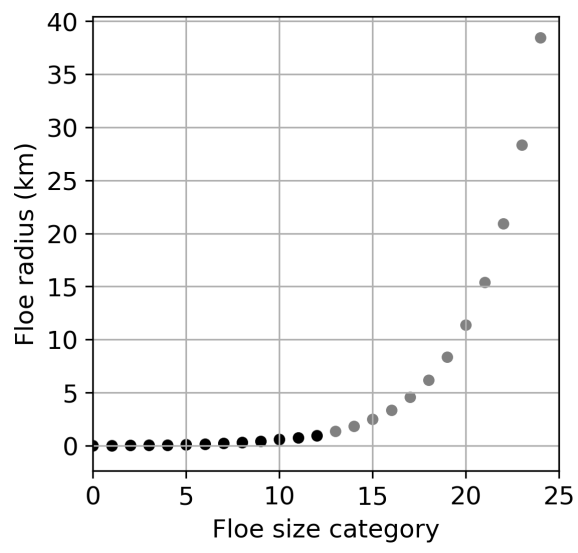

Figure C.1: The values of (black) standard and (grey) extended floe size radii category boundaries

plitudes may be very small in open water areas, wind effects mean they are unlikely be zero. The very large floes produced by this likely-artificial behaviour in the model can be broken up with the wave fracture scheme. All other processes tend to zero below floe sizes of $10 \mathrm{~km}$, in contrast to the runs with 12 categories where all processes save lateral growth are active at the largest category. This suggests that floe size categories do not need to extend beyond $10 \mathrm{~km}$.

Compared to FSD-v2 using 12 floe size categories only, extending floe size categories results in a smoother tendency arising from wave fracture in SH March, with net gains at all radii lower than around $100 \mathrm{~m}$. In both hemispheres, in both March and September, there is a transition in the net tendency in the number FSD due to wave fracture at around $100 \mathrm{~m}$ : below this, the net tendency is for floes to be produced by fracture, and above this, the net tendency is to be fractured. This indicates that the 'preferred sizes' arising from wave fracture discussed in Chapter 4, in runs with greater truncation of floe size categories, are in fact due to the artificial accumulation of floes in the largest size category. This reservoir of floes drives higher likelihood of obtaining floes at certain smaller categories. The net gains in smaller floe size categories due to fracture in the run with extended floe size categories are much smoother (Fig. C.2e-h), and floes below $100 \mathrm{~m}$ are less likely to be fractured. The existence of such a transition in the impact of wave fracture is in qualitative agreement with the findings of Toyota et al. (2006, 2011, 2016); Steer et al. (2008); Geise et al. (2017) and could be tested with targeted observations. 
(a) $\mathrm{NH}$ Mar

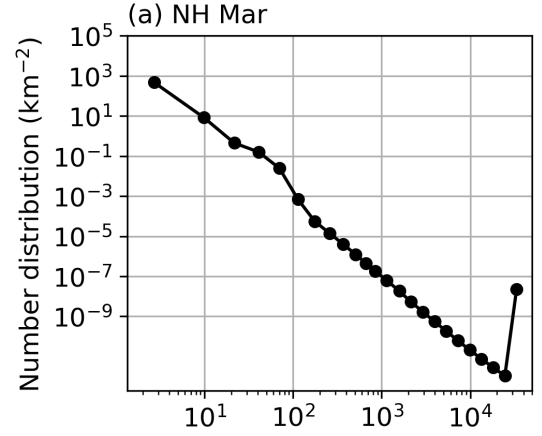

(b) NH Sep

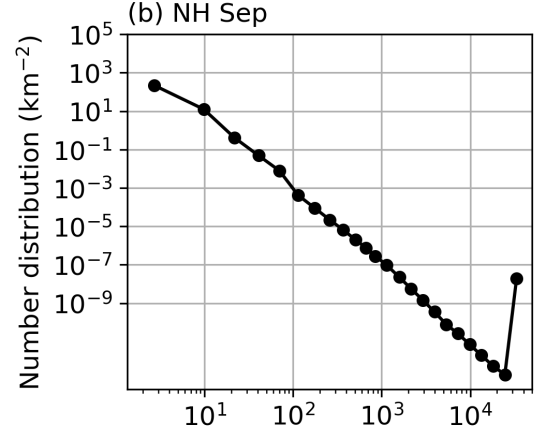

(c) $\mathrm{SH} \mathrm{Mar}$

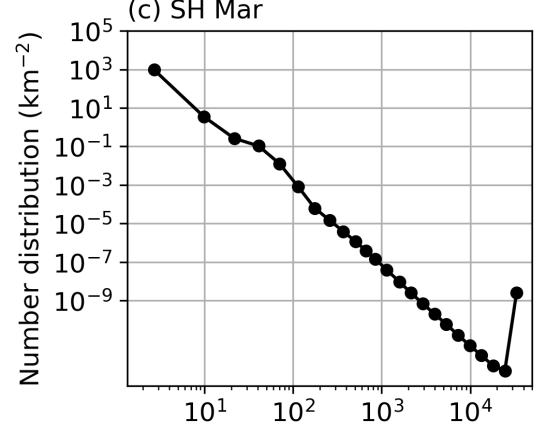

(d) SH Sep

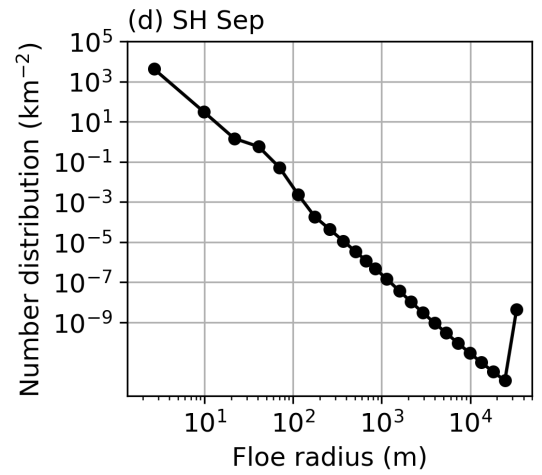

(e) NH Mar
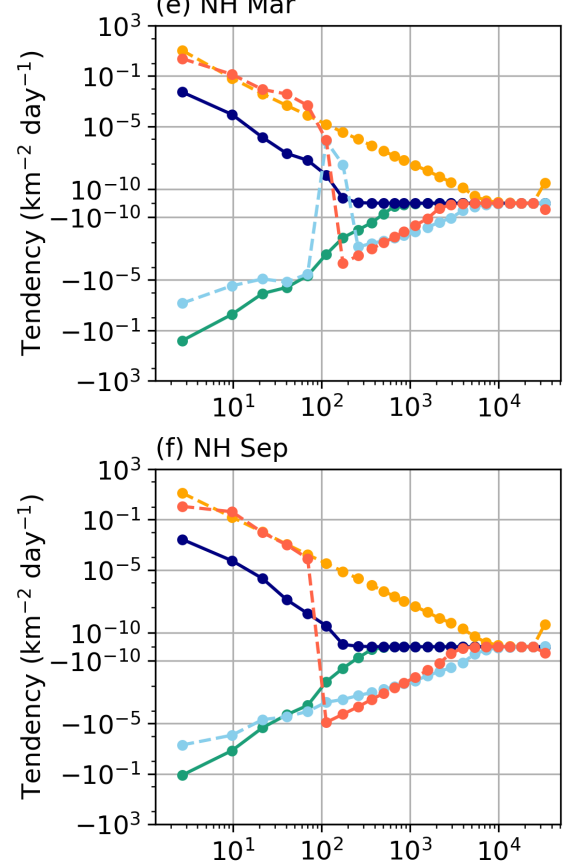

(g) $\mathrm{SH}$ Mar

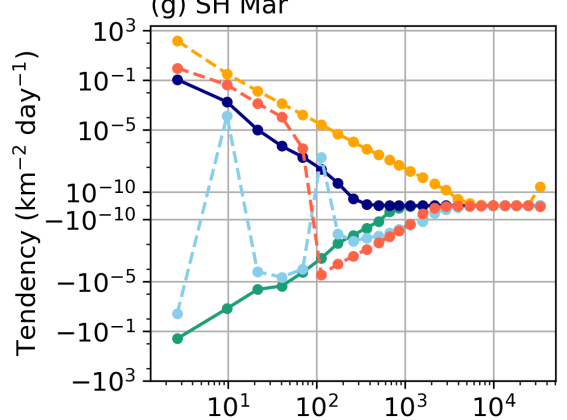

(h) SH Sep

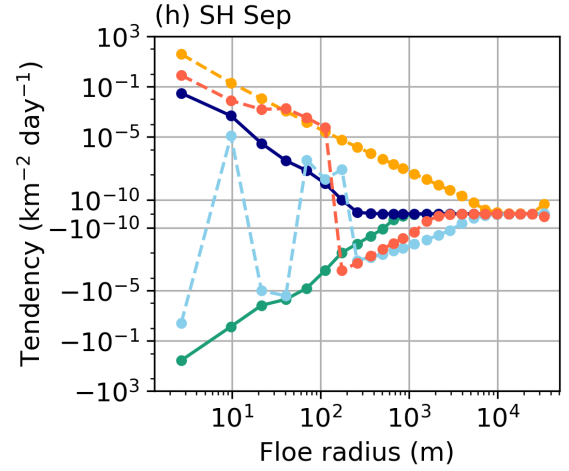

Figure C.2: (a) The Northern Hemisphere floe number distribution from the FSD-v2 climatological simulation where the floe size categories are extended in March averaged over ten years following model spin-up. (b-d) Same as (a), for the Northern Hemisphere in September, the Southern Hemisphere in March, and the Southern Hemisphere in September, respectively. (e) The net tendency in the floe number distribution from different physical processes in the Northern Hemisphere in March averaged over the twenty years following model spin-up. The axis in (e) is linearized around zero. (f-h) Same as (e), for the Northern Hemisphere in September, the Southern Hemisphere in March, and the Southern Hemisphere in September respectively. 


\section{Bibliography}

Aksenov, Y., E. E. Popova, A. Yool, A. G. Nurser, T. D. Williams, L. Bertino, and J. Bergh (2017), On the future navigability of Arctic sea routes: Highresolution projections of the Arctic Ocean and sea ice, Marine Policy, 75, 300-317, doi:10.1016/j.marpol.2015.12.027.

Alexander, M. A., U. S. Bhatt, J. E. Walsh, M. S. Timlin, J. S. Miller, and J. D. Scott (2004), The atmospheric response to realistic Arctic sea ice anomalies in an AGCM during winter, Journal of Climate, 17(5), 890-905, doi:10.1175/15200442(2004)017<0890:TARTRA >2.0.CO;2.

Andersen, S., R. Tonboe, S. Kern, and H. Schyberg (2006), Improved retrieval of sea ice total concentration from spaceborne passive microwave observations using numerical weather prediction model fields: An intercomparison of nine algorithms, Remote Sensing of Environment, 104(4), 374-392, doi:10.1016/j.rse.2006.05.013.

Arntsen, A. E., A. J. Song, D. K. Perovich, and J. A. Richter-Menge (2015), Observations of the summer breakup of an Arctic sea ice cover, Geophysical Research Letters, 42(19), 8057-8063, doi:10.1002/2015GL065224.

Asplin, M. G., R. Galley, D. G. Barber, and S. Prinsenberg (2012), Fracture of summer perennial sea ice by ocean swell as a result of Arctic storms, Journal of Geophysical Research: Oceans, 117(C06025), doi:10.1029/2011JC007221.

Asplin, M. G., R. Scharien, B. Else, S. Howell, D. G. Barber, T. Papakyriakou, and S. Prinsenberg (2014), Implications of fractured Arctic perennial ice cover on thermodynamic and dynamic sea ice processes, Journal of Geophysical Research: Oceans, 119(4), 2327-2343, doi:10.1002/2013JC009557.

Baede, A. P. M., E. Ahlonsou, Y. Ding, and D. Schimel (2001), The Climate System: An 
Overview, in Climate Change 2001: The Scientific Basis. Contribution of WGI to the 3rd Assessment Report, IPCC, Cambridge University Press.

Bennetts, L., and V. Squire (2012), Model sensitivity analysis of scatteringinduced attenuation of ice-coupled waves, Ocean Modelling, 45-46, 1-13, doi:10.1016/j.ocemod.2012.01.002.

Bennetts, L. G., S. O'Farrell, P. Uotila, and V. A. Squire (2014), Towards a model of the marginal ice zone for use in climate studies, in 22nd IAHR International Symposium on Ice, Singapore.

Bennetts, L. G., S. O'Farrell, and P. Uotila (2017), Brief communication: Impacts of ocean-wave-induced breakup of Antarctic sea ice via thermodynamics in a stand-alone version of the CICE sea-ice model, The Cryosphere, 11(3), 1035-1040, doi:10.5194/tc11-1035-2017.

Bentsen, M., I. Bethke, J. B. Debernard, T. Iversen, A. Kirkevåg, Ø. Seland, H. Drange, C. Roelandt, I. A. Seierstad, C. Hoose, and J. E. Kristjánsson (2013), The Norwegian Earth System Model, NorESM1-M - Part 1: Description and basic evaluation of the physical climate, Geoscientific Model Development, 6(3), 687-720, doi:10.5194/gmd6-687-2013.

Bitz, C. M., and W. H. Lipscomb (1999), An energy-conserving thermodynamic model of sea ice, Journal of Geophysical Research: Oceans, 104(C7), 15,669-15,677, doi:10.1029/1999JC900100.

Bitz, C. M., and G. H. Roe (2004), A mechanism for the high rate of sea ice thinning in the Arctic Ocean, Journal of Climate, 17(18), 3623-3632, doi:10.1175/15200442(2004)017<3623:AMFTHR>2.0.CO;2.

Bitz, C. M., M. M. Holland, A. J. Weaver, and M. Eby (2001), Simulating the icethickness distribution in a coupled climate model, Journal of Geophysical Research: Oceans, 106(C2), 2441-2463, doi:10.1029/1999JC000113.

Briegleb, B. P., and B. Light (2007), A Delta-Eddington multiple scattering parameterization for solar radiation in the sea ice component of the Community Climate System Model, Technical Note TN-472STR, National Center for Atmospheric Research, Boulder, CO, United States. 
Briegleb, B. P., C. M. Bitz, E. C. Hunke, W. H. Lipscomb, M. M. Holland, J. L. Schramm, and R. E. Moritz (2004), Scientific description of the sea ice component in the Community Climate System Model, version three, Technical Note TN-463STR, National Center for Atmospheric Research, Boulder, CO, United States.

Brooks, C. E. P. (1925), The problem of warm polar climates, Quarterly Journal of the Royal Meteorological Society, 51(214), 83-94.

Brooks, C. E. P. (1926), Climate Through the Ages: a Study of the Climatic Factors And Their Variations, R. V. Coleman, New York.

Bryan, K. (1969), Climate and the Ocean Circulation, Monthly Weather Review, 97(11), 806-827, doi:10.1175/1520-0493(1969)097<0806:CATOC>2.3.CO;2.

Bryan, K., S. Manabe, and R. C. Pacanowski (1975), A Global Ocean-Atmosphere Climate Model. Part II. The Oceanic Circulation, Journal of Physical Oceanography, 5, 30-46, doi:10.1175/1520-0485(1975)005<0030:AGOACM>2.0.CO;2.

Budyko, M. I. (1969), The effect of solar radiation variations on the climate of the Earth, Tellus, 21(5), 611-619, doi:10.1111/j.2153-3490.1969.tb00466.x.

Bunzel, F., D. Notz, J. Baehr, W. A. Muller, K. Frohlich, W. A. Müller, and K. Fröhlich (2016), Seasonal climate forecasts significantly affected by observational uncertainty of Arctic sea ice concentration, Geophysical Research Letters, 43(2), 852-859, doi:10.1002/2015GL066928.

Caesar, L., S. Rahmstorf, A. Robinson, G. Feulner, and V. Saba (2018), Observed fingerprint of a weakening Atlantic Ocean overturning circulation, Nature, 556(7700), 191-196, doi:10.1038/s41586-018-0006-5.

Caldeira, K., and I. Cvijanovic (2014), Estimating the contribution of sea ice response to climate sensitivity in a climate model, Journal of Climate, 27(22), 8597-8607, doi:10.1175/JCLI-D-14-00042.1.

Cavalieri, D. J., and C. L. Parkinson (2012), Arctic sea ice variability and trends, 19792010, The Cryosphere, 6(4), 881-889, doi:10.5194/tc-6-881-2012.

Cavalieri, D. J., P. Gloersen, and W. J. Campbell (1984), Determination of sea ice parameters with the NIMBUS 7 SMMR, Journal of Geophysical Research: Atmospheres, 89(D4), 5355-5369, doi:10.1029/JD089iD04p05355. 
CDO (2015), Climate Data Operators, Retrieved from https://github.com/itseez/opencv.

Clauset, A., C. R. Shalizi, and M. E. J. Newman (2009), Power-law distributions in empirical data, SIAM Review, 51(4), 661-703, doi:10.1137/070710111.

Cohen, J. L., J. C. Furtado, M. A. Barlow, V. A. Alexeev, and J. E. Cherry (2012), Arctic warming, increasing snow cover and widespread boreal winter cooling, Environmental Research Letters, 7(1), 014,007, doi:10.1088/1748-9326/7/1/014007.

Collins, M., R. Knutti, J. Arblaster, J. L. Dufresne, T. Fichefet, P. Friedlingstein, X. Gao, W. J. Gutowski, T. Johns, G. Krinner, M. Shongwe, C. Tebaldi, A. J. Weaver, and M. Wehner (2013), Long-term Climate Change: Projections, Commitments and Irreversibility Pages 1029 to 1076, in Climate Change 2013 - The Physical Science Basis, edited by Intergovernmental Panel on Climate Change, pp. 1029-1136, Cambridge University Press, Cambridge, doi:10.1017/CBO9781107415324.024.

Comiso, J. C. (1986), Characteristics of Arctic winter sea ice from satellite multispectral microwave observations, Journal of Geophysical Research, 91(C1), 975, doi:10.1029/JC091iC01p00975.

Comiso, J. C., D. J. Cavalieri, C. L. Parkinson, and P. Gloersen (1997), Passive microwave algorithms for sea ice concentration: A comparison of two techniques, Remote Sensing of Environment, 60(3), 357-384, doi:10.1016/S0034-4257(96)00220-9.

Coon, M. (1972), Mechanical Behavior of Compacted Arctic Ice Floes, in Offshore Technology Conference, Offshore Technology Conference, Houston, TX, United States, doi:10.4043/1684-MS.

Coon, M., R. Kwok, G. Levy, M. Pruis, H. Schreyer, and D. Sulsky (2007), Arctic Ice Dynamics Joint Experiment (AIDJEX) assumptions revisited and found inadequate, Journal of Geophysical Research, 112(C11), C11S90, doi:10.1029/2005JC003393.

Coon, M. D., G. A. Maykut, R. S. Pritchard, D. A. Rothrock, and A. S. Thorndike (1974), Modeling the pack ice as an elastic-plastic material, AIDJEX Bulletin, 24, 1-105.

Dansereau, V., J. Weiss, P. Saramito, and P. Lattes (2016), A Maxwell elasto-brittle rheology for sea ice modelling, The Cryosphere, 10(3), 1339-1359, doi:10.5194/tc-101339-2016. 
De Silva, L. W. A., H. Yamaguchi, and J. Ono (2015), Ice-ocean coupled computations for sea-ice prediction to support ice navigation in Arctic sea routes, Polar Research, 34(1), 25,008, doi:10.3402/polar.v34.25008.

Doble, M. J. (2009), Simulating pancake and frazil ice growth in the Weddell Sea: A process model from freezing to consolidation, Journal of Geophysical Research, 114(C9), C09,003, doi:10.1029/2008JC004935.

Doble, M. J., and P. Wadhams (2006), Dynamical contrasts between pancake and pack ice, investigated with a drifting buoy array, Journal of Geophysical Research, 111(C11), C11S24, doi:10.1029/2005JC003320.

Dumont, D., A. Kohout, and L. Bertino (2011), A wave-based model for the marginal ice zone including a floe breaking parameterization, Journal of Geophysical Research, 116(C4), C04,001, doi:10.1029/2010JC006682.

Ebert, E. E., and J. A. Curry (1993), An intermediate one-dimensional thermodynamic sea ice model for investigating ice-atmosphere interactions, Journal of Geophysical Research, 98(C6), 10,085, doi:10.1029/93JC00656.

Eisenman, I. (2010), Geographic muting of changes in the Arctic sea ice cover, Geophysical Research Letters, 37(16), L16,501, doi:10.1029/2010GL043741.

Feltham, D. L. (2005), Granular flow in the marginal ice zone, Philosophical Transactions of the Royal Society A: Mathematical, Physical and Engineering Sciences, 363(1832), 1677-1700, doi:10.1098/rsta.2005.1601.

Ferreira, D., J. Marshall, C. M. Bitz, S. Solomon, and A. Plumb (2015), Antarctic Ocean and Sea Ice Response to Ozone Depletion: A Two-Time-Scale Problem, Journal of Climate, 28(3), 1206-1226, doi:10.1175/JCLI-D-14-00313.1.

Fetterer, F., K. Knowles, W. Meier, M. Savoie, and A. K. Windnagel. (2017), Sea Ice Index, Version 3. Extent Anomaly Graphs, updated daily. Accessed 17/08/2018, NSIDC: National Snow and Ice Data Center, Boulder, CO, United States, doi:10.7265/N5K072F8.

Fichefet, T., and M. A. M. Maqueda (1997), Sensitivity of a global sea ice model to the treatment of ice thermodynamics and dynamics, Journal of Geophysical Research: Oceans, 102(C6), 12,609-12,646, doi:10.1029/97JC00480. 
Filbet, F., and P. Laurençot (2004), Numerical simulation of the Smoluchowski coagulation equation, SIAM Journal on Scientific Computing, 25(6), 2004-2028, doi:10.1137/S1064827503429132.

Flato, G., J. Marotzke, B. Abiodun, P. Braconnot, S. C. Chou, W. Collins, P. Cox, F. Driouech, S. Emori, V. Eyring, C. Forest, P. Gleckler, E. Guilyardi, C. Jakob, V. Kattsov, C. Reason, and M. Rummukainen (2013), Evaluation of Climate Models, in Climate Change 2013 - The Physical Science Basis, edited by Intergovernmental Panel on Climate Change, chap. 9, pp. 741-866, Cambridge University Press, Cambridge, doi:10.1017/CBO9781107415324.020.

Flato, G. M., and W. D. Hibler (1992), Modeling pack ice as a cavitating fluid, Journal of Physical Oceanography, 22(6), 626-651, doi:10.1175/15200485(1992)022<0626:MPIAAC >2.0.CO;2.

Flocco, D., D. L. Feltham, and A. K. Turner (2010), Incorporation of a physically based melt pond scheme into the sea ice component of a climate model, Journal of Geophysical Research, 115(C8), C08,012, doi:10.1029/2009JC005568.

Flocco, D., D. L. Feltham, E. Bailey, and D. Schroeder (2015), The refreezing of melt ponds on Arctic sea ice, Journal of Geophysical Research: Oceans, 120(2), 647-659, doi:10.1002/2014JC010140.

Francis, J. A., and S. J. Vavrus (2012), Evidence linking Arctic amplification to extreme weather in mid-latitudes, Geophysical Research Letters, 39(6), L06,801, doi:10.1029/2012GL051000.

Geise, G. R., C. C. Barton, and S. F. Tebbens (2017), Power scaling and seasonal changes of floe areas in the Arctic East Siberian Sea, Pure and Applied Geophysics, 174(1), 387-396, doi:10.1007/s00024-016-1364-2.

Gherardi, M., and M. C. Lagomarsino (2015), Characterizing the size and shape of sea ice floes, Scientific Reports, 5(1), 10,226, doi:10.1038/srep10226.

Goessling, H. F., S. Tietsche, J. J. Day, E. Hawkins, and T. Jung (2016), Predictability of the Arctic sea ice edge, Geophysical Research Letters, 43(4), 1642-1650, doi:10.1002/2015GL067232. 
Goosse, H., J. M. Campin, T. Fichefet, and E. Deleersnijder (1997), Impact of sea-ice formation on the properties of Antarctic bottom water, Annals of Glaciology, 25, 276281, doi:10.3189/S0260305500014154.

Gordon, C., C. Cooper, C. A. Senior, H. Banks, J. M. Gregory, T. C. Johns, J. F. B. Mitchell, and R. A. Wood (2000), The simulation of SST, sea ice extents and ocean heat transports in a version of the Hadley Centre coupled model without flux adjustments, Climate Dynamics, 16(2-3), 147-168, doi:10.1007/s003820050010.

Gray, J. M. N. T. (1999), Loss of hyperbolicity and ill-posedness of the viscous-plastic sea ice rheology in uniaxial divergent flow, Journal of Physical Oceanography, 29(11), 2920-2929, doi:10.1175/1520-0485(1999)029<2920:LOHAIP>2.0.CO;2.

Greenwood, N. N., and A. Earnshaw (2012), Chemistry of the Elements, 2nd ed., Butterworth-Heinemann.

Gregory, J. M. (2004), A new method for diagnosing radiative forcing and climate sensitivity, Geophysical Research Letters, 31(3), L03,205, doi:10.1029/2003GL018747.

Grenfell, T., and J. Comiso (1986), Multifrequency passive microwave observations of first-year sea ice grown in a tank, IEEE Transactions on Geoscience and Remote Sensing, GE-24(6), 826-831, doi:10.1109/TGRS.1986.289696.

Hartmann, J., C. Kottmeier, and C. Wamser (1992), Radiation and Eddy Flux Experiment 1991: (REFLEX I), Tech. rep., Berichte zur Polarforschung 105, Alfred Wegener Institute for Polar and Marine Research, Germany.

Herbers, T. H. C., P. F. Jessen, T. T. Janssen, D. B. Colbert, and J. H. MacMahan (2012), Observing ocean surface waves with GPS-tracked buoys, Journal of Atmospheric and Oceanic Technology, 29(7), 944-959, doi:10.1175/JTECH-D-11-00128.1.

Herman, A. (2010), Sea-ice floe-size distribution in the context of spontaneous scaling emergence in stochastic systems, Physical Review E, 81(6), 066,123, doi:10.1103/PhysRevE.81.066123.

Herman, A. (2016), Discrete-Element bonded-particle Sea Ice model DESIgn, version 1.3a - model description and implementation, Geoscientific Model Development, 9(3), 1219-1241, doi:10.5194/gmd-9-1219-2016. 
Herman, A., K.-U. Evers, and N. Reimer (2018), Floe-size distributions in laboratory ice broken by waves, The Cryosphere, 12(2), 685-699, doi:10.5194/tc-12-685-2018.

Hibler, W. D. (1979), A dynamic thermodynamic sea ice model, Journal of Physical Oceanography, 9(4), 815-846, doi:10.1175/15200485(1979)009<0815:adtsim >2.0.co;2.

Hibler, W. D. (1980), Modeling a variable thickness sea ice cover, Monthly Weather Review, 108(12), 1943-1973, doi:10.1175/15200493(1980)108<1943:MAVTSI >2.0.CO;2.

Hibler, W. D., and K. Bryan (1987), A diagnostic ice-ocean model, Journal of Physical Oceanography, 17(7), 987-1015, doi:10.1175/15200485(1987)017<0987:ADIM>2.0.CO;2.

Hobbs, W. R., R. Massom, S. Stammerjohn, P. Reid, G. Williams, and W. Meier (2016), A review of recent changes in Southern Ocean sea ice, their drivers and forcings, Global and Planetary Change, 143, 228-250, doi:10.1016/j.gloplacha.2016.06.008.

Holland, M. M., C. M. Bitz, and B. Tremblay (2006), Future abrupt reductions in the summer Arctic sea ice, Geophysical Research Letters, 33(23), L23,503, doi:10.1029/2006GL028024.

Holland, P. R., N. Bruneau, C. Enright, M. Losch, N. T. Kurtz, and R. Kwok (2014), Modeled trends in Antarctic sea ice thickness, Journal of Climate, 27(10), 3784-3801, doi:10.1175/JCLI-D-13-00301.1.

Holt, B., and S. Martin (2001), The effect of a storm on the 1992 summer sea ice cover of the Beaufort, Chukchi, and East Siberian Seas, Journal of Geophysical Research: Oceans, 106(C1), 1017-1032, doi:10.1029/1999JC000110.

Horvat, C., and E. Tziperman (2015), A prognostic model of the sea-ice floe size and thickness distribution, The Cryosphere, 9(6), 2119-2134, doi:10.5194/tc-9-2119-2015.

Horvat, C., and E. Tziperman (2017), The evolution of scaling laws in the sea ice floe size distribution, Journal of Geophysical Research: Oceans, 122(9), 7630-7650, doi:10.1002/2016JC012573. 
Horvat, C., E. Tziperman, and J.-M. Campin (2016), Interaction of sea ice floe size, ocean eddies, and sea ice melting, Geophysical Research Letters, 43(15), 8083-8090, doi:10.1002/2016GL069742.

Hunke, E. C., and J. K. Dukowicz (1997), An elastic-viscous-plastic model for sea ice dynamics, Journal of Physical Oceanography, 27(9), 1849-1867, doi:10.1175/15200485(1997)027<1849:AEVPMF>2.0.CO;2.

Hunke, E. C., W. H. Lipscomb, and A. K. Turner (2010), Sea-ice models for climate study: retrospective and new directions, Journal of Glaciology, 56(200), 1162-1172, doi:10.3189/002214311796406095.

Hunke, E. C., W. H. Lipscomb, A. K. Turner, N. Jeffery, and S. Elliott (2015), CICE: the Los Alamos Sea Ice Model Documentation and Software User's Manual Version 5.1, Technical Note LA-CC-06-012, Los Alamos National Laboratory, Los Alamos, United States.

Hwang, B., J. Wilkinson, E. Maksym, H. C. Graber, A. Schweiger, C. Horvat, D. K. Perovich, A. E. Arntsen, T. P. Stanton, J. Ren, and P. Wadhams (2017), Winter-tosummer transition of Arctic sea ice breakup and floe size distribution in the Beaufort Sea, Elementa: Science of the Anthropocene, 5, 40, doi:10.1525/elementa.232.

Inoue, J. (2004), Ice floe distribution in the Sea of Okhotsk in the period when sea-ice extent is advancing, Geophysical Research Letters, 31(20), L20,303, doi:10.1029/2004GL020809.

Itseez (2015), Open Source Computer Vision Library, Retrieved from https://github.com/itseez/opencv.

Ivanova, D. P., P. J. Gleckler, K. E. Taylor, P. J. Durack, and K. D. Marvel (2016), Moving beyond the total sea ice extent in gauging model biases, Journal of Climate, 29(24), 8965-8987, doi:10.1175/JCLI-D-16-0026.1.

Ivanova, N., O. M. Johannessen, L. T. Pedersen, and R. T. Tonboe (2014), Retrieval of Arctic sea ice parameters by satellite passive microwave sensors: A comparison of eleven sea ice concentration algorithms, IEEE Transactions on Geoscience and Remote Sensing, 52(11), 7233-7246, doi:10.1109/TGRS.2014.2310136. 
Ivanova, N., L. T. Pedersen, R. T. Tonboe, S. Kern, G. Heygster, T. Lavergne, A. Sørensen, R. Saldo, G. Dybkjær, L. Brucker, and M. Shokr (2015), Inter-comparison and evaluation of sea ice algorithms: towards further identification of challenges and optimal approach using passive microwave observations, The Cryosphere, 9(5), 1797-1817, doi:10.5194/tc-9-1797-2015.

Japan Meteorological Agency (2013), JRA-55: Japanese 55-year Reanalysis, Daily 3Hourly and 6-Hourly Data, Archive at the National Center for Atmospheric Research, Boulder, CO, United States, doi:10.5065/D6HH6H41.

Jones, N. (2009), Pancake ice takes over the Arctic, Nature Publishing Group, Retrieved from http://www.nature.com/doifinder/10.1038/news.2009.183, doi:10.1038/news.2009.183.

Josberger, E. G., and S. Martin (1981), A laboratory and theoretical study of the boundary layer adjacent to a vertical melting ice wall in salt water, Journal of Fluid Mechanics, 111(-1), 439, doi:10.1017/S0022112081002450.

Kaleschke, L., C. Lüpkes, T. Vihma, J. Haarpaintner, A. Bochert, J. Hartmann, and G. Heygster (2001), SSM/I sea ice remote sensing for mesoscale oceanatmosphere interaction analysis, Canadian Journal of Remote Sensing, 27(5), 526-537, doi:10.1080/07038992.2001.10854892.

Kay, J. E., C. Deser, A. Phillips, A. Mai, C. Hannay, G. Strand, J. M. Arblaster, S. C. Bates, G. Danabasoglu, J. Edwards, M. Holland, P. Kushner, J.-F. Lamarque, D. Lawrence, K. Lindsay, A. Middleton, E. Munoz, R. Neale, K. Oleson, L. Polvani, and M. Vertenstein (2015), The Community Earth System Model (CESM) Large Ensemble Project: A community resource for studying climate change in the presence of internal climate variability, Bulletin of the American Meteorological Society, 96(8), 1333-1349, doi:10.1175/BAMS-D-13-00255.1.

Killworth, P. D. (1983), Deep convection in the World Ocean, Reviews of Geophysics, 21(1), 1-26, doi:10.1029/RG021i001p00001.

Kohout, A. L., and M. H. Meylan (2008), An elastic plate model for wave attenuation and ice floe breaking in the marginal ice zone, Journal of Geophysical Research, 113(C9), C09,016, doi:10.1029/2007JC004434. 
Kohout, A. L., M. J. M. Williams, S. M. Dean, and M. H. Meylan (2014), Storminduced sea-ice breakup and the implications for ice extent, Nature, 509(7502), 604607, doi:10.1038/nature13262.

Komuro, Y., T. Suzuki, T. T. Sakamoto, H. Hasumi, M. Ishii, M. Wanatabe, T. Nozawa, T. Yokohata, T. Nishimura, K. Ogochi, S. Emori, and M. Kimoto (2012), Sea-ice in twentieth-century simulations by new MIROC coupled Models: A comparison between models with high resolution and with ice thickness distribution, Journal of the Meteorological Society of Japan, 90A, 213-232, doi:10.2151/jmsj.2012-A11.

Kottmeier, C., J. Hartmann, and C. Wamser (1994), Radiation and eddy flux experiment 1993:(REFLEX II), Tech. rep., Berichte zur Polarforschung 133, Alfred Wegener Institute for Polar and Marine Research, Germany.

Kreyscher, M., M. Harder, P. Lemke, and G. M. Flato (2000), Results of the Sea Ice Model Intercomparison Project: Evaluation of sea ice rheology schemes for use in climate simulations, Journal of Geophysical Research: Oceans, 105(C5), 11,299-11,320, doi:10.1029/1999JC000016.

Lecomte, O., H. Goosse, T. Fichefet, P. Holland, P. Uotila, V. Zunz, and N. Kimura (2016), Impact of surface wind biases on the Antarctic sea ice concentration budget in climate models, Ocean Modelling, 105, 60-70, doi:10.1016/j.ocemod.2016.08.001.

Leonard, G., H. H. Shen, and S. F. Ackley (1998), Dynamic growth of a pancake ice cover, in Proceedings of 14th International Symposium on Ice: Ice in Surface Waters, Potsdam, NY, United States.

Lipscomb, W. H. (2001), Remapping the thickness distribution in sea ice models, Journal of Geophysical Research: Oceans, 106(C7), 13,989-14,000, doi:10.1029/2000JC000518.

Lipscomb, W. H., and E. C. Hunke (2004), Modeling sea ice transport using incremental remapping, Monthly Weather Review, 132(6), 1341-1354, doi:10.1175/15200493(2004)132<1341:MSITUI>2.0.CO;2.

Lipscomb, W. H., E. C. Hunke, W. Maslowski, and J. Jakacki (2007), Ridging, strength, and stability in high-resolution sea ice models, Journal of Geophysical Research, 112(C3), C03S91, doi:10.1029/2005JC003355. 
Lu, P., Z. J. Li, Z. H. Zhang, and X. L. Dong (2008), Aerial observations of floe size distribution in the marginal ice zone of summer Prydz Bay, Journal of Geophysical Research, 113(C2), C02,011, doi:10.1029/2006JC003965.

Lund, B., H. C. Graber, P. O. G. Persson, M. Smith, M. Doble, J. Thomson, and P. Wadhams (2018), Arctic sea ice drift measured by shipboard marine radar, Journal of Geophysical Research: Oceans, 123(6), 4298-4321, doi:10.1029/2018JC013769.

Lüpkes, C., V. M. Gryanik, J. Hartmann, and E. L. Andreas (2012), A parametrization, based on sea ice morphology, of the neutral atmospheric drag coefficients for weather prediction and climate models, Journal of Geophysical Research: Atmospheres, 117(D13), D13,112, doi:10.1029/2012JD017630.

Mahlstein, I., P. R. Gent, and S. Solomon (2013), Historical Antarctic mean sea ice area, sea ice trends, and winds in CMIP5 simulations, Journal of Geophysical Research: Atmospheres, 118(11), 5105-5110, doi:10.1002/jgrd.50443.

Manabe, S., and R. J. Stouffer (1980), Sensitivity of a global climate model to an increase of CO 2 concentration in the atmosphere, Journal of Geophysical Research, 85(C10), 5529, doi:10.1029/JC085iC10p05529.

Marcq, S., and J. Weiss (2012), Influence of sea ice lead-width distribution on turbulent heat transfer between the ocean and the atmosphere, The Cryosphere, 6(1), 143-156, doi:10.5194/tc-6-143-2012.

Martin, S., and P. Kauffman (1981), A field and laboratory study of wave damping by grease ice, Journal of Glaciology, 27(96), 283-313, doi:10.3189/S0022143000015392.

Maslanik, J., J. Stroeve, C. Fowler, and W. Emery (2011), Distribution and trends in Arctic sea ice age through spring 2011, Geophysical Research Letters, 38(13), L13,502, doi:10.1029/2011GL047735.

Massonnet, F., P. Mathiot, T. Fichefet, H. Goosse, C. König Beatty, M. Vancoppenolle, and T. Lavergne (2013), A model reconstruction of the Antarctic sea ice thickness and volume changes over 1980-2008 using data assimilation, Ocean Modelling, 64, 67-75, doi:10.1016/j.ocemod.2013.01.003.

Maykut, G. A., and M. G. McPhee (1995), Solar heating of the Arctic mixed layer, Journal of Geophysical Research, 100(C12), 24,691, doi:10.1029/95JC02554. 
Maykut, G. A., and D. K. Perovich (1987), The role of shortwave radiation in the summer decay of a sea ice cover, Journal of Geophysical Research, 92(C7), 7032, doi:10.1029/JC092iC07p07032.

Maykut, G. A., and N. Untersteiner (1971), Some results from a time-dependent thermodynamic model of sea ice, Journal of Geophysical Research, 76(6), 1550-1575, doi:10.1029/JC076i006p01550.

McLaren, A. J., H. T. Banks, C. F. Durman, J. M. Gregory, T. C. Johns, A. B. Keen, J. K. Ridley, M. J. Roberts, W. H. Lipscomb, W. M. Connolley, and S. W. Laxon (2006), Evaluation of the sea ice simulation in a new coupled atmosphere-ocean climate model (HadGEM1), Journal of Geophysical Research, 111(C12), C12,014, doi:10.1029/2005JC003033.

Mellor, M. (1986), Mechanical behavior of sea ice, in The Geophysics of Sea Ice, pp. 165-281, Springer US, Boston, MA, doi:10.1007/978-1-4899-5352-0_3.

Meylan, M. H. (2002), Wave response of an ice floe of arbitrary geometry, Journal of Geophysical Research, 107(C1), 3005, doi:10.1029/2000JC000713.

Meylan, M. H., L. G. Bennetts, and A. L. Kohout (2014), In situ measurements and analysis of ocean waves in the Antarctic marginal ice zone, Geophysical Research Letters, 41(14), 5046-5051, doi:10.1002/2014GL060809.

Montiel, F., and V. A. Squire (2017), Modelling wave-induced sea ice break-up in the marginal ice zone, Proceedings of the Royal Society A: Mathematical, Physical and Engineering Science, 473(2206), 20170,258, doi:10.1098/rspa.2017.0258.

Montiel, F., V. A. Squire, and L. G. Bennetts (2016), Attenuation and directional spreading of ocean wave spectra in the marginal ice zone, Journal of Fluid Mechanics, 790, 492-522, doi:10.1017/jfm.2016.21.

Naughten, K. A., K. J. Meissner, B. K. Galton-Fenzi, M. H. England, R. Timmermann, and H. H. Hellmer (2018), Future projections of Antarctic ice shelf melting based on CMIP5 scenarios, Journal of Climate, 31(13), 5243-5261, doi:10.1175/JCLI-D-170854.1.

Neelin, J. D. (2010), Climate change and climate modeling, 1st ed., Cambridge University Press, Cambridge, United Kingdom. 
Nghiem, S., I. Rigor, P. Clemente-Colón, G. Neumann, and P. Li (2016), Geophysical constraints on the Antarctic sea ice cover, Remote Sensing of Environment, 181, 281292, doi:10.1016/j.rse.2016.04.005.

Nihashi, S., and K. I. Ohshima (2001), Relationship between ice decay and solar heating through open water in the Antarctic sea ice zone, Journal of Geophysical Research: Oceans, 106(C8), 16,767-16,782, doi:10.1029/2000JC000399.

Notz, D. (2014), Sea-ice extent and its trend provide limited metrics of model performance, The Cryosphere, 8(1), 229-243, doi:10.5194/tc-8-229-2014.

Notz, D. (2015), How well must climate models agree with observations?, Philosophical Transactions of the Royal Society A: Mathematical, Physical and Engineering Sciences, 373(2052), 20140,164, doi:10.1098/rsta.2014.0164.

Notz, D., F. A. Haumann, H. Haak, J. H. Jungclaus, and J. Marotzke (2013), Arctic sea-ice evolution as modeled by Max Planck Institute for Meteorology's Earth system model, Journal of Advances in Modeling Earth Systems, 5(2), 173-194, doi:10.1002/jame.20016.

O'Farrell, S. P. (1998), Investigation of the dynamic sea ice component of a coupled atmosphere-sea ice general circulation model, Journal of Geophysical Research: Oceans, 103(C8), 15,751-15,782, doi:10.1029/98JC00815.

Ohshima, K. I., and S. Nihashi (2005), A simplified ice-ocean coupled model for the Antarctic ice melt season, Journal of Physical Oceanography, 35(2), 188-201, doi:10.1175/JPO-2675.1.

Ohshima, K. I., S. Nihashi, and K. Iwamoto (2016), Global view of sea-ice production in polynyas and its linkage to dense/bottom water formation, Geoscience Letters, 3(1), 13, doi:10.1186/s40562-016-0045-4.

Onstott, R., P. Gogineni, A. Gow, T. Grenfell, K. Jezek, D. Perovich, and C. Swift (1998), Electromagnetic and physical properties of sea ice formed in the presence of wave action, IEEE Transactions on Geoscience and Remote Sensing, 36(5), 1764-1783, doi:10.1109/36.718644.

Paget, M., A. P. Worby, and K. J. Michael (2001), Determining the floe-size distribution of East Antarctic sea ice from digital aerial photographs, Annals of Glaciology, 33(1), 94-100, doi:10.3189/172756401781818473. 
Parkinson, C. L., and D. J. Cavalieri (2012), Antarctic sea ice variability and trends, 19792010, The Cryosphere, 6(4), 871-880, doi:10.5194/tc-6-871-2012.

Parkinson, C. L., and W. M. Washington (1979), A large-scale numerical model of sea ice, Journal of Geophysical Research, 84(C1), 311, doi:10.1029/JC084iC01p00311.

Parmerter, R. R., and M. D. Coon (1972), Model of pressure ridge formation in sea ice, Journal of Geophysical Research, 77(33), 6565-6575, doi:10.1029/JC077i033p06565.

Pellichero, V., J.-B. Sallée, C. C. Chapman, and S. M. Downes (2018), The Southern Ocean meridional overturning in the sea-ice sector is driven by freshwater fluxes, $\mathrm{Na}$ ture Communications, 9(1), 1789, doi:10.1038/s41467-018-04101-2.

Perovich, D. K. (2002), Aerial observations of the evolution of ice surface conditions during summer, Journal of Geophysical Research, 107(C10), 8048, doi:10.1029/2000JC000449.

Perovich, D. K., and K. F. Jones (2014), The seasonal evolution of sea ice floe size distribution, Journal of Geophysical Research: Oceans, 119(12), 8767-8777, doi:10.1002/2014JC010136.

Perovich, D. K., J. A. Richter-Menge, K. F. Jones, and B. Light (2008), Sunlight, water, and ice: Extreme Arctic sea ice melt during the summer of 2007, Geophysical Research Letters, 35(11), L11,501, doi:10.1029/2008GL034007.

Persson, P. O. G. (2002), Measurements near the Atmospheric Surface Flux Group tower at SHEBA: Near-surface conditions and surface energy budget, Journal of Geophysical Research, 107(C10), 8045, doi:10.1029/2000JC000705.

Persson, P. O. G. (2012), Onset and end of the summer melt season over sea ice: thermal structure and surface energy perspective from SHEBA, Climate Dynamics, 39(6), 1349-1371, doi:10.1007/s00382-011-1196-9.

Polyakov, I. V., A. V. Pnyushkov, M. B. Alkire, I. M. Ashik, T. M. Baumann, E. C. Carmack, I. Goszczko, J. Guthrie, V. V. Ivanov, T. Kanzow, R. Krishfield, R. Kwok, A. Sundfjord, J. Morison, R. Rember, and A. Yulin (2017), Greater role for Atlantic inflows on sea-ice loss in the Eurasian Basin of the Arctic Ocean, Science, 356(6335), 285-291, doi:10.1126/science.aai8204. 
Previdi, M., K. L. Smith, and L. M. Polvani (2015), How well do the CMIP5 models simulate the Antarctic atmospheric energy budget?, Journal of Climate, 28(20), 79337942, doi:10.1175/JCLI-D-15-0027.1.

Rae, J. G. L., H. T. Hewitt, A. B. Keen, J. K. Ridley, A. E. West, C. M. Harris, E. C. Hunke, and D. N. Walters (2015), Development of the Global Sea Ice 6.0 CICE configuration for the Met Office Global Coupled model, Geoscientific Model Development, 8(7), 2221-2230, doi:10.5194/gmd-8-2221-2015.

Rampal, P., J. Weiss, D. Marsan, R. Lindsay, and H. Stern (2008), Scaling properties of sea ice deformation from buoy dispersion analysis, Journal of Geophysical Research, 113(C3), C03,002, doi:10.1029/2007JC004143.

Rampal, P., J. Weiss, C. Dubois, and J.-M. Campin (2011), IPCC climate models do not capture Arctic sea ice drift acceleration: Consequences in terms of projected sea ice thinning and decline, Journal of Geophysical Research, 116(9), C00D07, doi:10.1029/2011JC007110.

Rinke, A., W. Maslowski, K. Dethloff, and J. Clement (2006), Influence of sea ice on the atmosphere: A study with an Arctic atmospheric regional climate model, Journal of Geophysical Research, 111(D16103), 1-14, doi:10.1029/2005JD006957.

Roach, L. A., S. F. B. Tett, M. J. Mineter, K. Yamazaki, and C. D. Rae (2017), Automated parameter tuning applied to sea ice in a global climate model, Climate Dynamics, 50(12), 51-65, doi:10.1007/s00382-017-3581-5.

Roach, L. A., S. M. Dean, and J. A. Renwick (2018a), Consistent biases in Antarctic sea ice concentration simulated by climate models, The Cryosphere, 12(1), 365-383, doi:10.5194/tc-12-365-2018.

Roach, L. A., C. Horvat, S. M. Dean, and C. M. Bitz (2018b), An emergent sea ice floe size distribution in a global coupled ocean-sea ice model, Journal of Geophysical Research: Oceans, 123(6), 4322-4337, doi:10.1029/2017JC013692.

Roach, L. A., M. M. Smith, and S. M. Dean (2018c), Quantifying growth of pancake sea ice floes using images from drifting buoys, Journal of Geophysical Research: Oceans, 123(4), 2851-2866, doi:10.1002/2017JC013693. 
Rösel, A., and L. Kaleschke (2012), Exceptional melt pond occurrence in the years 2007 and 2011 on the Arctic sea ice revealed from MODIS satellite data, Journal of Geophysical Research: Oceans, 117(C5), C05,018, doi:10.1029/2011JC007869.

Rosenblum, E., and I. Eisenman (2016), Faster Arctic sea ice retreat in CMIP5 than in CMIP3 due to volcanoes, Journal of Climate, 29(24), 9179-9188, doi:10.1175/JCLID-16-0391.1.

Rothrock, D. A. (1975), The energetics of the plastic deformation of pack ice by ridging, Journal of Geophysical Research, 80(33), 4514-4519, doi:10.1029/JC080i033p04514.

Rothrock, D. A., and A. S. Thorndike (1984), Measuring the sea ice floe size distribution, Journal of Geophysical Research, 89(C4), 6477, doi:10.1029/JC089iC04p06477.

Rottier, P. J. (1992), Floe pair interaction event rates in the marginal ice zone, Journal of Geophysical Research, 97(C6), 9391, doi:10.1029/92JC00152.

Rousset, C., M. Vancoppenolle, G. Madec, T. Fichefet, S. Flavoni, A. Barthélemy, R. Benshila, J. Chanut, C. Levy, S. Masson, and F. Vivier (2015), The Louvain-La-Neuve sea ice model LIM3.6: global and regional capabilities, Geoscientific Model Development, 8(10), 2991-3005, doi:10.5194/gmd-8-2991-2015.

Russell, G. L., J. R. Miller, and D. Rind (1995), A coupled atmosphere-ocean model for transient climate change studies, Atmosphere-Ocean, 33(4), 683-730, doi:10.1080/07055900.1995.9649550.

Rynders, S., Y. Aksenov, D. Feltham, G. Nurser, and A. Naveira Garabato (2016), Modelling MIZ dynamics in a global model, in EGU Gen. Assem. Conf. Abstr., p. 1004.

Salas Melia, D. (2002), A global coupled sea ice-ocean model, Ocean Modelling, 4(2), 137-172, doi:10.1016/S1463-5003(01)00015-4.

Schweiger, A., R. Lindsay, J. Zhang, M. Steele, H. Stern, and R. Kwok (2011), Uncertainty in modeled Arctic sea ice volume, Journal of Geophysical Research, 116(C8), C00D06, doi:10.1029/2011JC007084.

Screen, J. A. (2013), Influence of Arctic sea ice on European summer precipitation, Environmental Research Letters, 8(4), 044,015, doi:10.1088/1748-9326/8/4/044015. 
Screen, J. A., and I. Simmonds (2010), The central role of diminishing sea ice in recent Arctic temperature amplification, Nature, 464(7293), 1334-1337, doi:10.1038/nature09051.

Sellers, W. D. (1969), A global climatic model based on the energy balance of the earth-atmosphere system, Journal of Applied Meteorology, 8(3), 392-400, doi:10.1175/1520-0450(1969)008<0392:AGCMBO >2.0.CO;2.

Semtner, A. J. (1976), A model for the thermodynamic growth of sea ice in numerical investigations of climate, Journal of Physical Oceanography, 6(3), 379-389, doi:10.1175/1520-0485(1976)006<0379:AMFTTG >2.0.CO;2.

Semtner, A. J. (1984), On modelling the seasonal thermodynamic cycle of sea ice in studies of climatic change, Climatic Change, 6(1), 27-37, doi:10.1007/BF00141666.

Serreze, M. C., A. P. Barrett, J. C. Stroeve, D. N. Kindig, and M. M. Holland (2009), The emergence of surface-based Arctic amplification, The Cryosphere, 3(1), 11-19, doi:10.5194/tc-3-11-2009.

Sévellec, F., A. V. Fedorov, and W. Liu (2017), Arctic sea-ice decline weakens the Atlantic Meridional Overturning Circulation, Nature Climate Change, 7(8), 604-610, doi:10.1038/nclimate3353.

Shen, H., W. Perrie, Y. Hu, and Y. He (2018), Remote sensing of waves propagating in the marginal ice zone by SAR, Journal of Geophysical Research: Oceans, 123(1), 189-200, doi:10.1002/2017JC013148.

Shen, H. H. (2004), Limiting diameter of pancake ice, Journal of Geophysical Research, 109(C12), C12,035, doi:10.1029/2003JC002123.

Shen, H. H., and S. F. Ackley (1991), A one-dimensional model for wave-induced ice-floe collisions, Annals of Glaciology, 15(1), 87-95, doi:10.3189/1991AoG15-1-87-95.

Shen, H. H., and S. F. Ackley (1995), Laboratory-produced pancake ice cover in a twodimensional wave field, Antarctic Journal of the United States, 30(5), 106-108.

Shen, H. H., W. D. Hibler, and M. Leppäranta (1987), The role of floe collisions in sea ice rheology, Journal of Geophysical Research, 92(C7), 7085, doi:10.1029/JC092iC07p07085. 
Shen, H. H., S. F. Ackley, and M. A. Hopkins (2001), A conceptual model for pancake-ice formation in a wave field, Annals of Glaciology, 33(2), 361-367, doi:10.3189/172756401781818239.

Shu, Q., Z. Song, and F. Qiao (2015), Assessment of sea ice simulations in the CMIP5 models, The Cryosphere, 9(1), 399-409, doi:10.5194/tc-9-399-2015.

Smith, D. C. (2002), Arctic Ocean mixed-layer eddy generation under leads in sea ice, Journal of Geophysical Research, 107(C8), 3103, doi:10.1029/2001JC000822.

Smoluchowski, M. V. (1916), Zur Theorie der Zustandsgleichungen, Annalen der Physik, 353(24), 1098-1102, doi:10.1002/andp.19163532407.

Spreen, G., L. Kaleschke, and G. Heygster (2008), Sea ice remote sensing using AMSR-E 89-GHz channels, Journal of Geophysical Research, 113(C2), C02S03, doi:10.1029/2005JC003384.

Steele, M. (1992), Sea ice melting and floe geometry in a simple ice-ocean model, Journal of Geophysical Research: Oceans, 97(C11), 17,729-17,738, doi:10.1029/92JC01755.

Steele, M., J. H. Morison, and N. Untersteiner (1989), The partition of air-ice-ocean momentum exchange as a function of ice concentration, floe size, and draft, Journal of Geophysical Research, 94(C9), 12,739, doi:10.1029/JC094iC09p12739.

Steer, A., A. Worby, and P. Heil (2008), Observed changes in sea-ice floe size distribution during early summer in the western Weddell Sea, Deep Sea Research Part II: Topical Studies in Oceanography, 55(8-9), 933-942, doi:10.1016/j.dsr2.2007.12.016.

Steffen, K., and A. Schweiger (1991), NASA team algorithm for sea ice concentration retrieval from Defense Meteorological Satellite Program special sensor microwave imager: Comparison with Landsat satellite imagery, Journal of Geophysical Research, 96(C12), 21,971, doi:10.1029/91JC02334.

Stern, H. L., A. J. Schweiger, M. Stark, J. Zhang, M. Steele, and B. Hwang (2018a), Seasonal evolution of the sea-ice floe size distribution in the Beaufort and Chukchi seas, Elementa: Science of the Anthropocene, 6(1), 48, doi:10.1525/elementa.305.

Stern, H. L., A. J. Schweiger, J. Zhang, and M. Steele (2018b), On reconciling disparate studies of the sea-ice floe size distribution, Elementa: Science of the Anthropocene, 6(1), 49, doi:10.1525/elementa.304. 
Stroeve, J. C., V. Kattsov, A. Barrett, M. Serreze, T. Pavlova, M. Holland, and W. N. Meier (2012), Trends in Arctic sea ice extent from CMIP5, CMIP3 and observations, Geophysical Research Letters, 39(16), L16,502, doi:10.1029/2012GL052676.

Stroeve, J. C., S. Jenouvrier, G. G. Campbell, C. Barbraud, and K. Delord (2016), Mapping and assessing variability in the Antarctic marginal ice zone, pack ice and coastal polynyas in two sea ice algorithms with implications on breeding success of snow petrels, The Cryosphere, 10(4), 1823-1843, doi:10.5194/tc-10-1823-2016.

Talley, L. D., G. L. Pickard, W. J. Emery, and J. H. Swift (2011), Descriptive Physical Oceanography, 6th ed., Elsevier, Boston, United States, doi:10.1016/C2009-0-243224.

Taylor, K. E., R. J. Stouffer, and G. A. Meehl (2012), An overview of CMIP5 and the experiment design, Bulletin of the American Meteorological Society, 93(4), 485-498, doi:10.1175/BAMS-D-11-00094.1.

Tett, S. F. B., M. J. Mineter, C. Cartis, D. J. Rowlands, and P. Liu (2013), Can topof-atmosphere radiation measurements constrain climate predictions? Part I: Tuning, Journal of Climate, 26(23), 9348-9366, doi:10.1175/JCLI-D-12-00595.1.

Thomson, J. (2012), Wave breaking dissipation observed with "SWIFT" drifters, Journal of Atmospheric and Oceanic Technology, 29(12), 1866-1882, doi:10.1175/JTECH-D12-00018.1.

Thomson, J., and W. E. Rogers (2014), Swell and sea in the emerging Arctic Ocean, Geophysical Research Letters, 41(9), 3136-3140, doi:10.1002/2014GL059983.

Thomson, J., S. Ackley, F. Girard-Ardhuin, F. Ardhuin, A. Babanin, G. Boutin, J. Brozena, S. Cheng, C. Collins, M. Doble, C. Fairall, P. Guest, C. Gebhardt, J. Gemmrich, H. C. Graber, B. Holt, S. Lehner, B. Lund, M. H. Meylan, T. Maksym, F. Montiel, W. Perrie, O. Persson, L. Rainville, E. Rogers, H. Shen, H. Shen, V. Squire, S. Stammerjohn, J. Stopa, M. M. Smith, P. Sutherland, and P. Wadhams (2018), Overview of the Arctic Sea State and Boundary Layer Physics Program, Journal of Geophysical Research: Oceans, 123, 8674-8687, doi:10.1002/2018JC013766.

Thorndike, A. S., D. A. Rothrock, G. A. Maykut, and R. Colony (1975), The thickness distribution of sea ice, Journal of Geophysical Research, 80(33), 4501-4513, doi:10.1029/JC080i033p04501. 
Tilling, R. L., A. Ridout, and A. Shepherd (2018), Estimating Arctic sea ice thickness and volume using CryoSat-2 radar altimeter data, Advances in Space Research, 62(6), 1203-1225, doi:10.1016/j.asr.2017.10.051.

Timmermann, R. (2004), Utilizing the ASPeCt sea ice thickness data set to evaluate a global coupled sea ice-ocean model, Journal of Geophysical Research, 109(C7), C07,017, doi:10.1029/2003JC002242.

Tolman, H. L. (2009), User manual and system documentation of WAVEWATCH III TM version 3.14, Technical Note, MMAB Contribution, Environmental Modeling Center, NCEP, Camp Springs, MD, United States.

Tonboe, R. T., G. Dybkjaer, and J. L. Hoyer (2011), Simulations of the snow covered sea ice surface temperature and microwave effective temperature, Tellus A: Dynamic Meteorology and Oceanography, 63(5), 1028-1037, doi:10.1111/j.16000870.2011.00530.x.

Toyota, T., S. Takatsuji, and M. Nakayama (2006), Characteristics of sea ice floe size distribution in the seasonal ice zone, Geophysical Research Letters, 33(2), L02,616, doi:10.1029/2005GL024556.

Toyota, T., C. Haas, and T. Tamura (2011), Size distribution and shape properties of relatively small sea-ice floes in the Antarctic marginal ice zone in late winter, Deep Sea Research Part II: Topical Studies in Oceanography, 58(9-10), 1182-1193, doi:10.1016/j.dsr2.2010.10.034.

Toyota, T., A. Kohout, and A. D. Fraser (2016), Formation processes of sea ice floe size distribution in the interior pack and its relationship to the marginal ice zone off East Antarctica, Deep Sea Research Part II: Topical Studies in Oceanography, 131, 28-40, doi:10.1016/j.dsr2.2015.10.003.

Tsamados, M., D. L. Feltham, and a. V. Wilchinsky (2013), Impact of a new anisotropic rheology on simulations of Arctic sea ice, Journal of Geophysical Research: Oceans, 118(1), 91-107, doi:10.1029/2012JC007990.

Tsamados, M., D. L. Feltham, D. Schroeder, D. Flocco, S. L. Farrell, N. Kurtz, S. W. Laxon, and S. Bacon (2014), Impact of variable atmospheric and oceanic form drag on simulations of Arctic sea ice, Journal of Physical Oceanography, 44(5), 1329-1353, doi:10.1175/JPO-D-13-0215.1. 
Tsamados, M., D. Feltham, A. Petty, D. Schroeder, and D. Flocco (2015), Processes controlling surface, bottom and lateral melt of Arctic sea ice in a state of the art sea ice model, Philosophical Transactions of the Royal Society A: Mathematical, Physical and Engineering Sciences, 373(2052), 20140,167, doi:10.1098/rsta.2014.0167.

Tsujino, H., T. Motoi, I. Ishikawa, M. Hirabara, H. Nakano, G. Yamanaka, T. Yasuda, and H. Ishizaki (2010), Reference manual for the Meteorological Research Institute community ocean model (MRI. COM) version 3, Tech. rep., Meteorological Research Institute, Ibaraki Prefecture, Japan.

Turner, J., T. J. Bracegirdle, T. Phillips, G. J. Marshall, and J. S. Hosking (2013), An initial assessment of Antarctic sea ice extent in the CMIP5 models, Journal of Climate, 26(5), 1473-1484, doi:10.1175/JCLI-D-12-00068.1.

Turner, J., T. Phillips, G. J. Marshall, J. S. Hosking, J. O. Pope, T. J. Bracegirdle, and P. Deb (2017), Unprecedented springtime retreat of Antarctic sea ice in 2016, Geophysical Research Letters, 44(13), 6868-6875, doi:10.1002/2017GL073656.

Untersteiner, N. (1964), Calculations of temperature regime and heat budget of sea ice in the central Arctic, Journal of Geophysical Research, 69(22), 4755-4766, doi:10.1029/JZ069i022p04755.

Uotila, P., P. Holland, T. Vihma, S. Marsland, and N. Kimura (2014), Is realistic Antarctic sea-ice extent in climate models the result of excessive ice drift?, Ocean Modelling, 79, 33-42, doi:10.1016/j.ocemod.2014.04.004.

Vancoppenolle, M., T. Fichefet, H. Goosse, S. Bouillon, G. Madec, and M. A. M. Maqueda (2009), Simulating the mass balance and salinity of Arctic and Antarctic sea ice. 1. Model description and validation, Ocean Modelling, 27(1-2), 33-53, doi:10.1016/j.ocemod.2008.10.005.

Vaughan, D. G., J. C. Comiso, I. Allison, J. Carrasco, G. Kaser, R. Kwok, P. Mote, T. Murray, F. Paul, J. Ren, E. Rignot, O. Solomina, K. Steffen, and T. Zhang (2013), Observations: Cryosphere, in Climate Change 2013 - The Physical Science Basis, edited by Intergovernmental Panel on Climate Change, chap. 4, pp. 317-382, Cambridge University Press, Cambridge, doi:10.1017/CBO9781107415324.012. 
Vavrus, S. J. (1999), The response of the coupled Arctic sea ice-atmosphere system to orbital forcing and ice motion at $6 \mathrm{kyr}$ and $115 \mathrm{kyr}$ BP, Journal of Climate, 12(3), 873-896, doi:10.1175/1520-0442(1999)012<0873:TROTCA >2.0.CO;2.

Vihma, T. (2014), Effects of Arctic sea ice decline on weather and climate: A review, Surveys in Geophysics, 35(5), 1175-1214, doi:10.1007/s10712-014-9284-0.

Wadhams, P., M. A. Lange, and S. F. Ackley (1987), The ice thickness distribution across the Atlantic sector of the Antarctic Ocean in midwinter, Journal of Geophysical Research, 92(C13), 14,535, doi:10.1029/JC092iC13p14535.

Wadhams, P., G. Aulicino, F. Parmiggiani, P. O. G. Persson, and B. Holt (2018), Pancake ice thickness mapping in the Beaufort Sea from wave dispersion observed in SAR imagery, Journal of Geophysical Research: Oceans, 123(3), 2213-2237, doi:10.1002/2017JC013003.

Wang, Y., B. Holt, W. Erick Rogers, J. Thomson, and H. H. Shen (2016), Wind and wave influences on sea ice floe size and leads in the Beaufort and Chukchi Seas during the summer-fall transition 2014, Journal of Geophysical Research: Oceans, 121(2), 15021525, doi:10.1002/2015JC011349.

Washington, W., and C. Parkinson (2005), Introduction to Three-Dimensional Climate Modeling, 2nd ed., University Science Books, United States.

Weeks, W. F. S., and S. F. Ackley (1986), The growth, structure, and properties of sea ice, in The Geophysics of Sea Ice, vol. 146, edited by N. Untersteiner, pp. 9-164, Springer, Boston, MA.

Williams, T. D., L. G. Bennetts, V. a. Squire, D. Dumont, and L. Bertino (2013a), Wave-ice interactions in the marginal ice zone. Part 1: Theoretical foundations, Ocean Modelling, 71, 81-91, doi:10.1016/j.ocemod.2013.05.010.

Williams, T. D., L. G. Bennetts, V. A. Squire, D. Dumont, and L. Bertino (2013b), Wave-ice interactions in the marginal ice zone. Part 2: Numerical implementation and sensitivity studies along 1D transects of the ocean surface, Ocean Modelling, 71, 92101, doi:10.1016/j.ocemod.2013.05.011.

Winton, M. (2001), FMS sea ice simulator, GFDL Technical Document, Geophysical Fluid Dynamics Laboratory, Princeton, United States. 
Woods, A. W. (1992), Melting and dissolving, Journal of Fluid Mechanics, 239, 429-448, doi:10.1017/S0022112092004476.

Worby, A. P., and I. Allison (1991), Ocean-atmosphere energy exchange over thin, variable concentration Antarctic pack ice, Annals of Glaciology, 15(1990), 184-190, doi:10.3189/1991AoG15-1-184-190.

Wu, T., L. Song, W. Li, Z. Wang, H. Zhang, X. Xin, Y. Zhang, L. Zhang, J. Li, F. Wu, Y. Liu, F. Zhang, X. Shi, M. Chu, J. Zhang, Y. Fang, F. Wang, Y. Lu, X. Liu, M. Wei, Q. Liu, W. Zhou, M. Dong, Q. Zhao, J. Ji, L. Li, and M. Zhou (2014), An overview of BCC climate system model development and application for climate change studies, Acta Meteorologica Sinica, 28(1), 34-56, doi:10.1007/s13351-014-3041-7.

Yakovlev, N. G. (2003), Coupled model of ocean general circulation and sea ice evolution in the Arctic Ocean, Izvestiya Atmospheric and Oceanic Physics, 39(3), 355-368.

Zhang, J., A. Schweiger, M. Steele, and H. Stern (2015), Sea ice floe size distribution in the marginal ice zone: Theory and numerical experiments, Journal of Geophysical Research: Oceans, 120(5), 3484-3498, doi:10.1002/2015JC010770.

Zhang, J., H. Stern, B. Hwang, A. Schweiger, M. Steele, M. Stark, and H. C. Graber (2016), Modeling the seasonal evolution of the Arctic sea ice floe size distribution, Elementa: Science of the Anthropocene, 4(1), 126, doi:10.12952/journal.elementa.000126.

Zhao, J., A. Bower, J. Yang, X. Lin, and N. Penny Holliday (2018), Meridional heat transport variability induced by mesoscale processes in the subpolar North Atlantic, Nature Communications, 9(1), 1124, doi:10.1038/s41467-018-03134-X.

Zunz, V., H. Goosse, and F. Massonnet (2013), How does internal variability influence the ability of CMIP5 models to reproduce the recent trend in Southern Ocean sea ice extent?, The Cryosphere, 7(2), 451-468, doi:10.5194/tc-7-451-2013.

Zweng, M., J. Reagan, J. Antonov, R. Locarnini, A. Mishonov, T. Boyer, H. Garcia, O. Baranova, D. Johnson, D. Seidov, and M. Biddle (2013), Volume 2: Salinity, in World Ocean Atlas, edited by S. Levitus and A. Mishonov, p. 39, NOAA. 